\title{
On Computational Ecosystems in Media Arts
}

\author{
Rui Filipe Nicolau Lima Antunes
}

Thesis submitted for the degree of Doctor of Philosophy

of the

University of London

Goldsmiths, University of London

December, 2013 


\section{DECLARATION}

I hereby declare that I composed this thesis entirely myself and that it describes my own research.

Rui Filipe Nicolau Lima Antunes

10th of October, 2013 
This research is an exploration of issues surrounding the artistic production of Computational Ecosystems. A Computational Ecosystem is a system of agents designed to emulate, in the computer, biological systems where autonomous individuals are organized in a hierarchical food chain and interact by trading units of energy. This thesis maps out this field and examines the modes of production and functions of these systems. The central claim is focused on how the narratives normally associated with these systems and their functioning are two complementary, but separate entities. By virtue of considering these separately the computational ecosystem is argued to be an abstract generative engine for heterogeneity, spontaneity, and even novelty. It is contended that the set of methods of production developed by exploratory artists using these artefacts might be instrumentalized as generative methods for the animation of general purpose non-player characters in virtual worlds. 
Keywords: virtual worlds; computational ecosystems; non-player characters; computational models of animation; generative art; evolutionary art; persistence. 


\section{Acknowledgments}

This project would be unthinkable without the routine support from supervisors, colleagues and friends, as well as the contribution of specialist knowledge by a host of professionals who were not only willing to be questioned about their experience and professional practice but were also enthusiastic about the prospect. I owe indebted to many people, far more than I can name here. First and foremost I owe gratitude to my supervisors, Professor Frederic Fol Leymarie, Professor William Latham and Professor Janis Jefferies, who guided this project with tremendous verve and thoughtfulness. Frederic was indefatigable in the endless meetings and revisions of the written material, William has never failed to produce provocative discussions, and Janis was always there when I needed her the most. I am very grateful to the 'usual suspects': my office mates, Mohammad al Rifaie and Patrick Tresset, and colleagues from seminars on Arts and Computational Technologies, who have been willing to discuss ideas, offer support and invariably lift my spirits with their energy and industry. I am equally grateful to those who initially supported and encouraged this project of research, such as art critic João Pinharanda and Professor Pedro Faria Lopes. João in particular provided an extraordinary impulse, inviting me to participate on the exhibition Lá fora. I owe thanks to Dr. Tim Taylor and Dr. Mick Grieson, who provided me with helpful pointers and offered most appreciated insights during the examination to transfer onto the $\mathrm{PhD}$ track.

Among the circle of experts and practitioners that provided me with valuable information, I especially would like to thank Professor James Lovelock, Professor Jansen Vincent, Professor Iwan Jones, and Dr. Stefano Allesina, who have provided many valuable insights from their areas of expertise; and also thank Professor Jon McCormack, Dr. Alan Dorin, Professor Larry Yager, Dr. Tim Taylor, Dr. Alice Eldridge, Dr. Cefn Hoile and Dr. Graham Wakefield for the feedback on their work.

Goldsmiths, and in particular the Computing Department, provided a comfortable base from which I was able to work on this project. Additionally, in the first year, the college awarded me a grant partially reducing the fees. This research was supported in the form of a PhD studentship by Fundação para a Ciência e Tecnologia in Portugal, contract reference SFRH /BD /61293 /2009. The work Where is Lourenço Marques? was also developed with the 
financial assistance of Fundación Telefónica under the award Incentives for Ibero-American Production from the VIDA competition, and the technical assistance of Vy Phan, Rui Leitão, Teresa Cardoso, and several anonymous participants. The editing skills of Joanna Pylak were invaluable in all the videos for xTNZ, Senhora da Graça and Where is Lourenço Marques?.

I owe a completely different debt to my family, and it is to them that I dedicate this thesis. Love and thanks to Joanna, in whom kindness and generosity are personified, for keeping me company and so much more. 


\section{Contents}

1 Introduction $\quad 16$

1.1 Motivations ............................ 16

1.2 Hypothesis and questions . . . . . . . . . . . . . . . . . 19

1.3 Methodology: ........................ 21

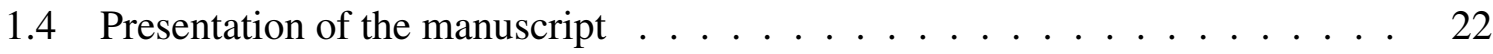

1.5 Public diffusion of the work .................... 25

2 From Animats to Crowds 28

2.1 Computational ecosystems . . . . . . . . . . . . . . 30

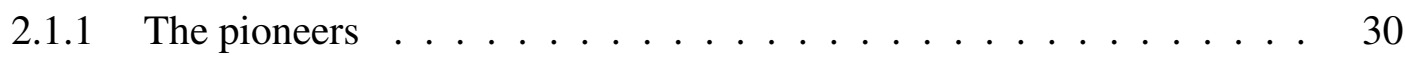

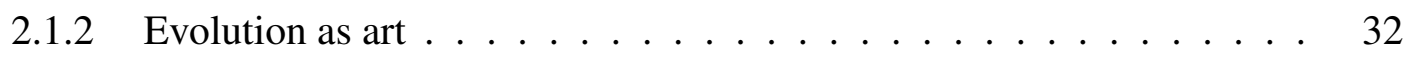

2.1.3 The artistic practice using CEs . . . . . . . . . . . . . . . 34

2.1.3.1 The new paradigm provided by sonic ecosystems . . . . 37

2.1.3.2 The 'remediation' of the technology of the CE in Virtual worlds . . . . . . . . . . . . . . 38

2.1.4 Behavioural animation of groups of non-player characters . . . . . 39

2.1.5 The problem of the persistence of virtual living populations . . . . . 41

2.1.6 The context of WisLM: Evolutionary Art and Relational Aesthetics . . 43

2.1.7 Final notes .................... 44

3 The Materiality of Evolutionary Art 46

3.1 Introduction . . . . . . . . . . . . . . . 46

3.2 Biots and digital aquaria of life: AI techniques informing the construction of computational ecosystems . . . . . . . . . . . . . . 46

3.2.1 The first class: Gtype-Ptype . . . . . . . . . . . . . . . . 47

3.2.2 Cellular automata, the second class . . . . . . . . . . . 50

3.2.3 The third class: co-evolution in multi-agent-systems . . . . . . . . 51

3.2.4 Other important techniques . . . . . . . . . . . . . 55 
3.3 Interactive aquaria: A participative audience . . . . . . . . . . . 57

3.4 Final notes . . . . . . . . . . . . . . . . . . . . . 60

4 Computational Ecosystems as Cultural Forms $\quad 61$

4.1 Introduction . . . . . . . . . . . . . . . . 61

4.2 Aesthetic affiliations of Evolutionary Art . . . . . . . . . . . . . 61

4.2.1 Open systems . . . . . . . . . . . . . . . 62

4.2.2 An aesthetics of self-organization and feedback . . . . . . . . . 67

4.3 The meaning of life in the systems . . . . . . . . . . . . . . 68

4.3 .1 Life forms . . . . . . . . . . . . . . . . 69

4.3.2 A narrative construct . . . . . . . . . . . . . 73

4.4 Final notes . . . . . . . . . . . . . . . . . . . . . . . . . . 74

5 The Functioning of Computational Ecosystems 76

5.1 Introduction . . . . . . . . . . . . . . . . . 76

5.2 A narrative construction . . . . . . . . . . . . . . . . 79

5.3 Case studies ........................... 86

5.3.1 Senhora da Graça . . . . . . . . . . . . . . . 87

5.3.2 Vishnu's Dance of Life and Death . . . . . . . . . . . . . . . 89

5.4 Final notes . . . . . . . . . . . . . . . . . . . . . . . . . . 90

6 A Comparative Study Towards Persistent Computational Ecosystems 92

6.1 Introduction . . . . . . . . . . . . . . . . . . . . . 92

6.2 Characterization of the problem . . . . . . . . . . . . . 93

6.2.1 Homogeneous model $(\mathrm{Hm}) \ldots \ldots . \ldots$. . . . . . . . 94

6.2.2 Chemostat model $(\mathrm{C}) \ldots \ldots \ldots$. . . . . . . . . . . . 94

6.2 .3 Heterogeneous model $(\mathrm{Ht}) \ldots \ldots$. . . . . . . . . . . 95

6.2 .4 Food web model $(\mathrm{F}) \ldots \ldots . \ldots . \ldots 95$

6.2.5 Other forms of organization . . . . . . . . . . . . . 98

6.3 Methodology . . . . . . . . . . . . . . . . . 99

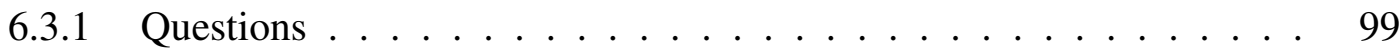

6.3 .2 Architecture ....................... 100

6.3.2.1 The sub-models . . . . . . . . . . . . . 100

6.3 .3 Experiments ....................... 101

6.3.3.1 Algorithms . . . . . . . . . . . . . . 101

6.3.3.2 Duration and dimension of the trials . . . . . . . . 104 
6.3.3.3 Sensitivity measurements . . . . . . . . . . . 105

6.4 Results . . . . . . . . . . . . . . . . . . . . 105

6.4.1 Presentation of the results . . . . . . . . . . . . 105

6.4.1.1 Comparison of approaches and methods . . . . . . . . . 108

6.4.1.2 Question 2: Is the predictive strategy better than the rescue ones? . . . . . . . . . . . . . . . . 109

6.4 .2 Discussion of the results . . . . . . . . . . . . . 110

6.4 .3 Final notes . . . . . . . . . . . . . . . . 113

6.5 Conclusions . . . . . . . . . . . . . . . . . . 114

7 Where is Lourenço Marques? - I 116

7.1 Introduction . . . . . . . . . . . . . . . . . 116

7.2 Context: Mozambique 1974-76 . . . . . . . . . . . . . . . . 117

7.3 Methodology: Chasing stories . . . . . . . . . . . . . . . . . 119

7.4 Discussion . . . . . . . . . . . . . . . . . . . . . . 124

8 Where is Lourenço Marques? - II 130

8.1 Introduction . . . . . . . . . . . . . . . . . . . 130

8.1.1 The computational ecosystem as an AI engine . . . . . . . . . . . 132

8.2 Description of the system . . . . . . . . . . . . . . . . 133

8.2.1 The agent representation . . . . . . . . . . . . . 133

8.3 Results . . . . . . . . . . . . . . . . . . . . . . . 142

8.4 Discussion and future work . . . . . . . . . . . . . . . . . . 147

8.5 Final notes . . . . . . . . . . . . . . . . . 153

9 Conclusions $\quad 155$

$\begin{array}{ll}\text { Bibliography } & 162\end{array}$ 


\section{List of Figures}

2.1 Still image from $x T N Z$, an ecosystem of evolutionary creatures whose physical features (shape and sounds) evolve in a generational time scale by means of a software architecture inspired by Mendelian genetics and Darwinian natural selection [19]. . . . . . . . . . . . . . . . . . . . . .

2.2 Left: A reinterpretation of the famous work of Leonardo DaVinci the Vitruvian Man, using a CE. A community of dynamic and 'starving' pixels eat sections of a digital representation of the drawing based on the hue value of the other static pixels, which become resources. Right: A similar process is at the origin of the Halley series, a series of dynamic works where the digital representations of the paintings from Peter Halley appear reinterpreted by means of the CE. . . . . . . . . . . . . . . . . . . . . .

2.3 Some examples of population changes in natural ecosystems. a) Song Thrush in English Farmlands, b) Lynx in the Canadian Artic, c) Bupulus, a moth in German forests, d) Collared Dove in England, e) Spotted Flycatcher in English farmlands, and f) Whitethroat in English farmlands. Scales: the abscissa in all cases is in years. The units of density are relative ones in (a), (d), (e) and (f), and are set to 100 in either 1966 (a), (e) and (f) or in 1972, (d). In (b) the units are thousands trapped per year and in (c) numbers per hectare of forest floor.

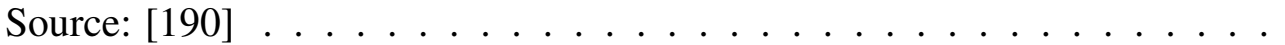

5.1 Still image from Senhora da Graça, where a population of plants, herbivores and carnivores evolves. . . . . . . . . . . . . . . . .

5.2 Still image from Vishnu's Dance of Life and Death, a choreography for NPCs animated with a CE. . . . . . . . . . . . . . . . . . .

6.1 Four distinct models of population, from left to right: Homogeneous (Hm); Chemostat (C); Heterogeneous (Ht); and Food web (F). 
6.2 From left to right: a) 25 runs with model pushing the demographics down to extinction, and b) 25 runs with model pushing the demographics up to overpopulation.

6.3 Left: Active algorithm (ACT): Individuals are forcibly inserted when the population numbers are below the lower threshold. By contrast, whenever the population goes above the superior threshold, the individuals with less energy are removed.

Right: Passive algorithm (INH): Insertions are forbidden above the carrying capacity. Removals are equally inhibited below the minimum threshold. . . .

6.4 With predictive method, the estimations are based on the local tendency of growth. In the example on the left, the local growth indicates a growth, whereas on the right a decrease in density is predicted.

6.5 The mean (from 50 runs) of the number of artificial actions produced using each of the methods under scrutiny after 100 cycles. From top to bottom: a) populations with strong intrinsic pressure towards overpopulation, and b) populations with strong intrinsic pressure towards extinction. . . . . . . . . . . . . . . . . 106

6.6 The mean (from 50 runs) of the number of artificial actions produced using each of the methods under scrutiny after 1.000 cycles. From top to bottom: a) populations with strong intrinsic pressure towards overpopulation, and b) populations with strong intrinsic pressure towards extinction. . . . . . . . . . . . . .

6.7 The mean (from 50 runs) of the number of artificial actions produced using each of the methods under scrutiny after 10.000 cycles. From top to bottom: a) populations with strong intrinsic pressure towards overpopulation, and b) populations with strong intrinsic pressure towards extinction. . . . . . . . . . . . . . . . .

6.8 The mean (from 50 runs) of the number of artificial actions produced using each of the methods under scrutiny after 100.000 cycles. From top to bottom: a) populations with strong intrinsic pressure towards overpopulation, and b) populations with strong intrinsic pressure towards extinction. . . . . . . . . . . . . . . . . . . 108

7.1 Different modes of representation coexist in WisLM. Here, a storyteller walks by a photograph of a pair of former 'Laurentinos' generously shared by the participants.

7.2 Diagram from a section of the city provided by one of the participants. . . . . 119 
7.3 Schematic representation of the virtual world showing the different layers of representation. From the bottom to the top, and from left to right: a) Shared schematic drawings; b) Sculpted topography departing from the descriptions and materials; c) Shared maps; d) Road layouts after the originals extracted from the maps; e) Sculpted buildings from the interpretation of 2d-images; f) $3 \mathrm{~d}$ and photographic representations of the same site appear juxtaposed; g) Virtual museum with documents; h) Music originating from the question "what is the first song that arises when you think about the city?"; i) Images of 'retornados' removed from their background; $j$ ) autonomous population of NPCs; k) storyteller. . . . . . . . . . . . . . . . . . . . .

7.4 Two kids staring at the audience from a distant past. The original black and white photography is from a shared personal photo-album. . . . . . . . . . . 123

7.5 WisLM draws on the tension of juxtaposing multiple modes of representation. Here the $2 \mathrm{D}$ and the $3 \mathrm{D}$ versions of the train station appear in the same point of view. . . . . . . . . . . . . . . . . . . . . 126

8.1 Overview of the virtual world. In the background are some buildings and billboards, and closer, some members of the community of storytellers in interaction. . . . . . . . . . . . . . . . . . . 130

8.2 Image of a 'storyteller' in interaction with the audience. . . . . . . . . . . . 132

8.3 Graph depicting the GType influence on the behaviour of the character. The variables (gIntr, gCycl, gUptk) directly influence the hormonal system, which motivates the actions performed in the world; the variables (gChem, gMetab) set the body-composition and dietary specifications, which determine the environmental context for the actions.

8.4 Illustration of a situation where the active messages Msg1 and Msg3 are generated due to the hypothetical simultaneous activation of sensors S1 and S3 in the perception state. The list of current active-messages is updated with the two new messages. Msg1 increases the priority of the existing entry for Msg1, whereas Msg3 updates the list with a new entry with priority $0 . . . . . .$.

8.5 An illustration of the process of transformation from message to action in the decision state. The message 000\#01 finds two compatible indexes matching in the table of rules (000001 and 000101). From these, the action of the one with highest priority is performed. 
8.6 Graphic depicting the overall structure of the algorithm. At the bottom, the action selection mechanism is illustrated: sensors trigger messages indicating a specific need; these messages are prioritized and form a buffer, which is ordered by associated priority. The message with the highest priority is selected to trigger an action. At the top of the graph, the mechanism rendering actions is illustrated. For each syntactic action, an associated animation is played. . .

8.7 Still images of the animations corresponding to each of the actions performed by the agent. Above, from left to right: a) eat prey; b) attempt to mate with a partner that is not ready; c) reproduction or play alone; d) successful mating; e) attempt to mate but no compatible individuals exist in the vicinity. Below, from left to right: i) losing an attack; ii) play with happy mate; iii) victorious attack; iv) walking (move to mate, wander, move to prey); . . . . . . . . . . . 140

8.8 Entropy of the community. The graph illustrates the cumulative frequency of genotypes present in the community since the start of the run.

8.9 Spatial distribution in time. At intervals of 15 seconds, a snapshot of the system was captured during a period of 6 hours (one day of exhibition). Each dot represents the location at that particular moment of one individual. The variables $\mathrm{x}$ and $\mathrm{z}$ stand for the coordinates in the Cartesian horizontal space. Each graph illustrates a frame of juxtaposed 15 minutes of execution.

8.10 Heterogeneity of actions performed. The graph illustrates the number of times each of the actions was performed. At intervals of 15 seconds, the system recorded what each individual was doing in that moment of time. The heterogeneity of the actions performed is represented from left to right by Attack, Collide, Eat, Hungry, HungryCr (search for Carcasses), HungryGj (search for other creatures), Move, MoveAway, Play, Reproduce, TryMate, Wander. . . .

8.11 Population density. The graph depicts the temporal evolution of the demographic dimension of the community. Each unit in the time scale (the abscissas) denotes 15 seconds. The vertical axis denotes the number of inhabitants.

8.12 Detailed screen-shot from the website capturing the moment a storyteller tells her account with visible subtitles in Portuguese (top) and English (bottom). 153

9.1 Diagrammatic summary of the context of the works. . . . . . . . . . . 196

9.2 Diagrammatic summary of the Interactivity of the works. . . . . . . . . . . 200

9.3 Diagrammatic summary of the formal presentation. . . . . . . . . . . . . . 204 
9.4 Histogram depicting results from interviews with the audience. From top to bottom and from left to right, the figures indicate the respective associated question. The dashed line indicates the mean; the overlaied kernel shows the density curve. The abscisses indicate the rating provided by the respondents in a scale from 1 to 10 . The axis of the ordinates indicate the density of the answers in the sample. . . . . . . . . . . . . . . . . 215

9.5 Images from the catalogue of the exhibition 'Lá fora', a perspective of Portuguese artists living and working abroad. The exhibition featured the work Senhora da Graça. . . . . . . . . . . . . . . . . . . . . . . . . 217

9.6 Image from the exhibition of WisLM at the closing ceremony of Watermans Festival of Digital Art 2012. I was part of the panel, 'Virtual space, Game space as Artistic Medium' - Chaired by Jonathan Munro - with Joseph Farbrook, Corrado Morgana, Parag K Mital, to talk about the work exhibited. . . 218

9.7 Images from the solo exhibition of WisLM at the Tin Shed, Goldsmiths from 29th November - 14th December 2012. The exhibition was accompained, in the opening session, by a panel with Amal Khalaf, Catarina Valdigem Pereira and myself on 'The role of collective memory in the construction of community identity and sense of place'. 


\section{Nomenclature}

AI Artificial Intelligence

ALife Artificial Life

ALife Artificial Life

ANN Artificial Neural Network

CA Cellular Automaton

CE Computational Ecosystem

EP Evolutionary Programming

ES Evolutionary Strategies

EvoArt Evolutionary Art

GA Genetic Algorithms

GenArt Generative Art

Gtype Genotype

NPC Non Player Character

Ptype Phenotype

Vishnu's Vishnu's Dance of Life and Death

VW Virtual World

WisLM Where is Lourenço Marques? 


\section{Introduction}

\subsection{Motivations}

\section{Personal interest}

In the early 1990s, I was a member of the BBS (Bulletin board system) named Visus where one evening some obscure 'geeky' telematic friend passed me some code in PASCAL. The wonder and excitement when this computer program first ran on my 386 CPU powered computer is still a vivid memory. It was a simple community of yellow dots feeding on green dots. These would turn red before turning off, or more precisely, as the program specified: 'died' Eventually the computer was turned off, but I still remember my hesitations and dilemma about 'killing' the community that I had set 'alive' minutes before.

Around the same period of time, in South California, a community of pioneers was gathering in Santa Fe to debate and promote the possibilities of emulating and creating life with computers, in the early steps of ALife as a scientific discipline in its own right. ALife stands for Artificial Life, that is a branch of Artificial Intelligence, in which interests lie in mimicking processes of natural life and evolution as found in carbon-based living forms. This was the time when cyberpop-culture emerged, potentialized by the massification of personal computers and the internet. It was also a period when the research on the genomic domain began to extensively map body functions and features with their associated genes, discrete units of information in the chromosome which would operate in a binary logic, thus providing a computational paradigm at the foundations of life.

The artistic manifestation par excellence of this culture was an art form sharing the techniques of ALife, if not its entire ethos: Evolutionary Art. This is a branch of Generative art, born in those utopian days, in which the system is characterized by the use of ALife techniques as a foundational generative processual approach. (This will be expanded in the next chapter.) The instrumentalization of principles of biological evolution for aesthetic purposes (in silico) is seen referred in literature as artificial life art, genetic art, evolving art, evolutionary art or organic $\operatorname{art}^{1}$. Throughout this document, I will refer to this practice as EvoArt. As Boden and

\footnotetext{
${ }^{1}$ A note to mention that on the lines above a conscious distinction is made with BioArt. This also makes use of
} 
Edmond emphasize, the focus of this methodology is on the evolution by Natural Selection observed in nature [44]: 'the artwork is evolved by processes of random variation and selective reproduction that affect the art-generating program itself'.

Authors are interested in capturing a moment in the evolution of the system, or the whole evolutionary process itself. Whitelaw categorizes three main dominant methodologies in this artistic genre using software as a medium and artificial life as a technique: Breeding, Cybernatures and Abstract works. According to this categorization, breeders are those who employ genetic strategies to produce visuals, such as Latham and Todd's FormGrowth [139]; cybernatures encompass the sub-genre of works involving the creation of ecologies in silico, such as Christa Sommerer's A-Volve [225]. IMA Traveler from Driessens \& Verstappen provides an example of abstract works [85, 251].

A similar allure attracted me a few years ago to these practices and, in my rolls of student of computing and of visual artist, I have developed an interest for these forms and methodologies and created $x T N Z$ - a self portrait [19], an evolutionary ecosystem; an artwork where the generative procedure used to create formal diversity was driven by the autonomous interactions of a population of autonomous individuals. This system inaugurated many of the avenues I am pursuing today, and that will be discussed throughout this document.

\section{Domain of knowledge}

Computational ecosystems (CEs) are multi-agent systems in which software agents are: represented by genetic-like blueprints, trade units of 'energy' and 'mass', are organized in a hierarchical manner as in a food chain in a 'carbon-based' ecosystem and emulate metabolic cycles (these might include birth, growing morphologies, reproduction and death).

My study is situated in the cross-disciplinary intersection of three different areas: Computer Science, the Visual Arts and Ecology. The territory this research maps is circumvented on the epistemological domain by four fundamental titles: Creative Evolutionary Systems edited by Peter Bentley and David Corne [36]; The Art of Evolutionary Art, edited by Penousal Machado et al. [210]; Virtual Worlds edited by Stefan Bornhofen et al. [49]; and Metacreations authored by Mitchell Whitelaw [251]. The first two are compilations of technical articles on Evolutionary Art. The third offers a collection of technical articles on dynamic virtual environments populated by virtual entities. The last offers a comprehensive study on ALife-Art, including the cultural aspects of this practice. In a book chapter of the second of these titles, Alan Dorin attempted to characterize a sub-field of ALife-Art and Evolutionary art, an art form using

evolutionary principles for aesthetic purposes (refer to the work of Eduardo Kac for instance), however using bio-chemical and carbon based entities rather than silicon. 
systems like $x T N Z$ : Computational Ecosystems. The central aim of the present research is to refine this field even more, mapping out this practice and provide a deeper understanding on the functioning of these systems.

\section{Objectives}

This research looks at these systems from the point of view of their use as artistic instruments. This perspective entails scrutinizing the operations involved in creating these objects, looking at these artefacts both as technical constructs and as cultural forms. I want to engage in a formal discussion that extends the modes of construction and functioning of these artefacts, approaching this technology in its entirety, without privileging the technological over the human, and simultaneously identify and minimize their current problems.

CEs may also be seen referred in the literature as creative ecosystems [163], systems used with a manifest interest in their generative properties as producers of dynamics, heterogeneity and, arguably, novelty. This interest in the generative features of these systems has some tradition in music [90] and audiovisual artistic works [158]. By means of analyzing this artistic practice, I want to understand how this methodology can be widened to other areas (primarily, but not exclusively artistic). Namely, I want to emphasize the $\mathrm{CE}$ as a generative tool used in the animation of Virtual Worlds (VWs).

In short, the main objective for this study can be stated simply as follows: to provide a solid contribution for the enrichment of generative art. Firstly, by mapping out this territory; secondly, by suggesting the 'remediation'2 of the CE in VWs by proposing it as a technique for the animation of general purpose Non-player characters (NPCs) ${ }^{3}$ in VWs.

To achieve these goals I will:

- scrutinize existing modes of artistic production using the $\mathrm{CE}$ as a supporting generative technology;

- look at CEs from the point of view of their materiality, by examining the set of techniques and conventions used to organize the data and structure of these artefacts;

\footnotetext{
${ }^{2}$ Remediation is a term suggested by Bolter and Grusin in Remediation: Understanding New Media to address the incorporation of older mediums in 'new' mediums. I am using a loose understanding of the term (in the broad lines of Marshal McLuhan's use of the term medium, for whom the focus is on the social function of the technology, and thus the boundaries between media and technology appear blurred. For instance, in Understanding Media he uses the term medium with regards to the light bulb, since this is a technological vector of social change: 'a light bulb creates an environment by its mere presence'). Following their terminology, it is said that the VW hyper-mediates the 'old' CE when this is absorbed, refashioned and incorporated.

${ }^{3}$ Non player characters are autonomous characters with different degrees of agency which appear in a game scenario.
} 
- describe CEs' modes of functioning by deconstructing their processes of authorship and audience reception;

- analyze and suggest a heuristic for the problem of the persistence of virtual populations.

\section{Contributions to Knowledge}

As a result, the essence of this research is essentially multidisciplinary and the contributions provided emerge in different domains of knowledge. These will be discussed in the next chapters and can be summarized as follows:

- On the technical side, VW's architectures get enhanced with the formalization of a new model for the development of populated virtual environments (presented in Chapter 8);

- Furthermore, this research also has the merit of building an analysis on computational ecosystems from the point of view of their persistence (I take up this point further in chapter 6).

- In the aesthetic realm, this research contributes a technical and cultural framework for the use of CEs as instruments for artistic practice;

- Last but not least, it provides new contexts for evolutionary art, hybridizing generative practices with a perspective more centered on inter-human relations, the human stories that these works may represent or produce (I take this point further in chapters 5 and 7).

\subsection{Hypothesis and questions}

This research may also be described as a Framework. This is due to its nature overviewing and mapping out this practice, providing methods and tools for practitioners. Departing from the construction of a case-study, it raises and answers a series of fundamental questions which expand or interfere with the development of CEs in VWs.

I argue that there is a set of production methods originated in the artistic practice using CEs, which allows an expansion of this practice, using these systems as generative methods in the animation of general purpose non-player characters in VWs. In essence, the incorporation of the technology of CEs into VWs is advocated. VWs are characterized by a constant movement of technological integration and consequent expansion. The 'remediation' of the CE in VWs brings to these everything we traditionally associate with CEs: Standard CEs are models depicting ecologies with ecosystem dynamics of the type: the carnivores eat the herbivores. 
Some authors, however, explore the inherent dynamics of these systems to generate audio (or audio-visual) pieces $[52,90]$. This thesis draws on this latter and more utilitarian approach, and explores the instrumentalization of the $\mathrm{CE}$ for other purposes rather than the direct visualization of the (virtual) ecologies. The technology being used for the animation of communities of humanoid avatars in VWs is one such applications.

\section{hypothesis \#1:}

Can CEs be used as generative instruments to animate individual and group behaviour in communities of general purpose NPCs in VWs? The design of Where is Lourenço Marques? (from now on referred as: WisLM - a work discussed in detail in chapters 7 and 8) has required the existence of a population of humanoid NPCs inhabiting in permanence the virtual environment. The design specifications also required the behaviours exhibited by this community to be driven by the dynamics of a CE. These assumptions raise the first question that this research tries to answer.

Pioneers of EvoArt, such as William Latham or Steven Rooke, have established the roots for the artistic genre by generating imagery exploring the paradigm provided by the Genetic Algorithm from Evolutionary Computing. In this methodology, a syntactic element (the Gtype) is converted into a semantic component (the Ptype). These artists have shown that the linearity and the distance of the process of conversion from the syntactic to the semantic levels of the EvoArt artwork may have multiple interpretations [251], and it is upon this space of possibilities that artists (mostly) intervene. William Latham produces $3 \mathrm{~d}$ morphologies based on a process of shape deformation, while Steven Rooke generates abstract imagery based on a language of mathematical and visual operators (this will be discussed in chapter 3). Following these footsteps, this thesis argues that this methodology can also be extended to the dynamics of CEs. A similar interpretative process may be applied not only to the physicality of the individuals, but also to the actions occurring in the ecosystem. Ephemeral events such as the actions of 'attack' or 'eat' might be considered also to be syntactic elements, and be decoded creatively in the semantic level. In an identical way, these actions might have, as well, more or less direct translations. The action 'attack' might be visualized literally rendered as a primal animation of a lion devouring some prey, or more freely as a dancer on stage approaching another performer while executing a specific dance movement. It is up to the artists to play with this space of freedom creatively. A few instances exist where artists use the ecosystem dynamics to sonify their works $[90,79,248]$. I will expand on their work and use this methodology to animate communities of humanoid characters in dancing (Chapt. 5) and talking activities 
(Chapt. 8).

\section{hypothesis \#2:}

Can a heuristic be found to maintain a steady density of population in a dynamic computational ecosystem? The second question is related to the first one and it originates in the same work. During the development of WisLM, a critical problem has arisen. Due to constraints imposed by the medium itself, namely its limited rendering capacity in real-time, the population inhabiting the virtual space had to be small in number (less than 200 simultaneous individuals $)^{4}$. Ecology tells us about the importance played by energetic pathways of the food web in the stability of the ecosystems $[224,86,11]$. However, on the other hand, it also tells us that such small populations are prone to stochastic extinctions [63], and that (large) systems with random correlations between species are typically unstable [155]. As a consequence, a heuristics to maintain stable populations was investigated: the Eco-A-Life model (This investigation is described in chapter 6).

By stable, I am referring to the persistence of the system, the robustness towards the introduction/invasion of new species, and the resiliency or ability to cope with extinctions. Can we implement a computational open system where the energetic pathways formed by the food web are emergent from the autonomous dynamics of the system, and can this system live in a state of non-equilibrium and be simultaneously stable?

In short, this thesis is a study on the technology of the $\mathrm{CE}$ as a generative instrument for novelty, heterogeneity and spontaneity in VWs, in particular as a generative technique for real-time animation of general purpose NPCs. This is an exploration that touches a variety of topics, but fundamentally this can be reduced to two interconnected main ideas: a) The CE as a generative technology used in animations of VWs; b) The formalization of a heuristic to maintain stable, persistent populations in VWs.

\subsection{Methodology:}

In 2009, the competition in art and artificial life, VIDA 12.0, organized by Spanish Fundación Telefónica, awarded me with a commission to produce the artwork WisLM. This work is a representation of the former city of Lourenço Marques in Mozambique, taking expression in a

\footnotetext{
${ }^{4}$ Of course there are other practical and established solutions such as sectioning the world so that only a section is visible at a time. However, I wanted to address this problem in a straight forward way.
} 
VW. It is an ethnographic project of representation dealing with questions on post-colonialism (the motivations for this work are described at length in chapter 7).

WisLM plays a critical role in the present investigation. It functions as the main tool for inquiry, motivating and triggering multiple questions for analysis. The adopted methodology of investigation is art as a mode of inquiry, where artistic works are the means to problematize, postulate questions and establish analytic dialogues. WisLM will serve as our guide, and it is upon its implementation that I will demonstrate the usefulness and practicability of these hypotheses.

Two more works were developed to illustrate certain aspects or ideas more obscure in the text: Senhora da Graça and Vishnu's Dance of Life and Death. Their development provides an opportunity to produce a process of deconstruction that problematizes technical/cultural aspects and makes possible an analysis of this discipline.

\subsection{Presentation of the manuscript}

This manuscript is divided into a small number of chapters. Each of these articulates with the practical work and covers one or a few of the key conceptual aspects under discussion. The manuscript is organized in a top-down approach, departing from contextual issues and successively refining the discussion to particular aspects under analysis. This is accomplished in two parts: in the first (chapters 2 to 5 of this manuscript), a literature review scrutinizes the field; in the second part (chapters 6 to 8), the CE is discussed as a technology for the animation of VWs with some technical aspects being analyzed in great detail.

Chapter 1: Introduction This is the current introductory chapter. The main lines of research are presented and the chapter is divided into small sections. The chapter explains the main motivations for the research, the aims and the objectives, its main hypothesis, and lists the contributions provided towards knowledge of this area. Additionally, the methodology adopted for research is presented, and a summarized description of the diffusion of knowledge is listed.

Chapter 2: From Animats to crowds: a literature review on the art of computational ecosystems The second chapter provides a context outlining the argument and main areas of contribution. This literature review is complemented with the remaining manuscript, where some aspects of the production are further expanded. The chapter starts by looking at EvoArt and its methods in order to argue these as useful in the animation of groups of NPCs 
in virtual environments. Following, a portrait of the main current approaches to behavioural animation of such groups is presented. Then, I look at some of the critical aspects of these systems, bringing some references found in the literature about the need for a heuristic to persistent CEs. The chapter ends by shifting its focus, by bringing attention to ways of enriching this art practice with an argument on the expansion of EvoArt's dialectics. A greater articulation of this artistic practice with relational aesthetics is suggested.

\section{Chapter 3: The Materiality of Evolutionary Art: Artificial Life Models in Soft-} ware Some aspects I found useful to look at are the tools of production, organization and configuration. This chapter analyzes the materiality of the CE. From a historical review on EvoArt, I will suggest that in the construction of CEs there is a convergence of older techniques that emerge from this artistic genre. EvoArt itself draws on techniques borrowed from ALife to explore evolutionary principles for the purpose of novelty - a feature that distinctly characterizes this artistic practice. In a literature review on EvoArt, I will look at the main techniques characterizing this practice. Each of these techniques is illustrated with artistic works where the specific technique plays a critical role. In the process, three technological paradigms converging in current standards of CEs' construction are suggested.

Chapter 4: Computational Ecosystems as cultural forms This chapter examines the cultural framework surrounding the artistic practice using CEs. I take a closer look at the strong and weak claims of artificial life to see how these notions about life came to inform the praxis of the art using CEs. In particular, this chapter emphasizes how these ideas articulate with the aesthetic affiliations of EvoArt in Generative art; how self-organization and emergence appear to be central objects of discourse and are omnipresent in the works' formulations and methods.

\section{Chapter 5: The functioning of Computational Ecosystems: On Writing and} Reading Artistic Computational Ecosystems The discussion on the workings of the CEs wouldn't be complete without interrogating the modes of functioning of these systems: Why is it that we say that a red individual is eating a green one when we see two dots flickering on the screen in a simulation? How do these systems construct meaning? The term 'system story' is borrowed from Mitchell Whitelaw to help us describe the particular model of narrative at play in these systems. This is expanded in a discussion that starts by looking at CEs as cultural artefacts, and invokes a number of voices to assist on this project. These include some of the key practitioners and cultural theorists, such as Katherine Hayles or Bruno Latour. This 
project expands in a twofold analysis: a) emphasizing the gap between the workings of the system from its visual representation; and b) deconstructing its narrative process.

Chapter 6: Persistence of Computational Ecosystems The community of NPCs (which forms the CE) plays a central role as storytellers in WisLM. This, however, has created some challenges in terms of the development of the work. On one hand, the design of the work drew upon an allegory that required the existence of an ecosystem dynamic, with its typical predatory behaviours and the consequent fluctuations in population density. On the other hand, due to its central role as 'storytellers', it was required that this population would have to be persistent. This aspect led me on an exploration of the mechanisms at play in existing CEs to maintain these within tractable densities. This also led me to look at the field of Ecology in order to develop a heuristic addressing this issue.

This chapter describes these studies, which laid out the foundational body for formulation of a model for persistence of populations in virtual ecosystems. I examine the impact of different strategies for persistence of small populations in digital ecosystems to conclude that existing strategies are not neutral and interfere with the natural evolution of the community. One heuristic is then suggested to deal with wild fluctuations in the population density in computational ecosystems. This suggested heuristic minimizes the number and size of the interventions required to assure milder fluctuations, however it still impacts the natural evolution of the community.

Chapter 7: Where is Lourenço Marques?: I - Motivations The ethnographic work Where is Lourenço Marques? is the main catalyst for this research and functions as the main motivation and causa finalis. This thesis is a discussion on questions risen during its preparation and implementation. This chapter initially describes the motivations providing a small historical introduction. Then, it progresses with a discussion on mediated memory and how this work enriches EvoArt.

\section{Chapter 8: Where is Lourenço Marques?: II - An Ecosystem Based Model for Real-time Generative Animation of Humanoid NPCs This chapter describes the decentralized autonomous model of agency for general purpose NPCs (the AI model of the $\mathrm{CE}$ ) used to animate the population of gregarious humanoid avatars in the virtual world Where is Lourenço Marques?. It first situates this work in the context of the technologies of crowd simulation, and then describes the implementation of the CE. This is a model where individ- uals have states driven by 'hormones'. The changes in their values trigger actions, which are}


translated into animations of humanoid characters socializing. The last section of the chapter describes the implementation of the $\mathrm{CE}$ as a generative engine producing the animation, as well as its articulation with the workings of the Eco-A-Life model for persistence, introduced in chapter 6.

Chapter 9: Conclusions This is a summary of the manuscript and presentation of the main conclusions of this research, and its future avenues.

\subsection{Public diffusion of the work}

\begin{tabular}{|c|c|c|c|}
\hline \multicolumn{4}{|c|}{ ARTWORKS, EXHIBITS } \\
\hline Lá Fora & $\begin{array}{c}\text { Museu da } \\
\text { Electricidade, } \\
\text { Lisbon, Portugal }\end{array}$ & 2010 & $\begin{array}{l}\text { Exhibition of Senhora da Graça in a } \\
\text { retrospective of contemporary and historical } \\
\text { Portuguese artists living abroad, organized by } \\
\text { the Presidency of the Portuguese Republic, } \\
\text { and curated by art critic, João Pinharanda. }\end{array}$ \\
\hline $\begin{array}{c}\text { Watermans } \\
\text { Festival of Digital } \\
\text { Arts }\end{array}$ & $\begin{array}{c}\text { Watermans Centre, } \\
\text { London }\end{array}$ & 2012 & $\begin{array}{l}\text { Exhibition of Where is Lourenço Marques? } \\
\text { as part of the show at the closing ceremony } \\
\text { of the festival. During the Festival, I also } \\
\text { took part in a panel entitled Art in Virtual } \\
\text { Reality /virtual spaces, Game-space as an } \\
\text { Artistic Medium, to talk about Where is } \\
\text { Lourenço Marques?. }\end{array}$ \\
\hline $\begin{array}{c}\text { Where is Lourenço } \\
\text { Marques? }\end{array}$ & $\begin{array}{c}\text { Tin Shed Gallery, } \\
\text { Goldsmiths, } \\
\text { London }\end{array}$ & 2013 & $\begin{array}{l}\text { Solo exhibition of Where is Lourenço } \\
\text { Marques?. During the opening event, a panel } \\
\text { discussed The role of collective memory in } \\
\text { the construction of community identity and } \\
\text { sense of place. This event was curated by } \\
\text { Jonathan Munro and Catherine Weir. }\end{array}$ \\
\hline
\end{tabular}




\begin{tabular}{|c|c|}
\hline \multicolumn{2}{|r|}{ CONFERENCE PAPERS } \\
\hline $\begin{array}{l}\text { Senhora Da Graça, a Virtual } \\
\text { World as Memorial [20] }\end{array}$ & $\begin{array}{l}\text { Short paper where the materiality and representational aspects of } \\
\text { virtual worlds were emphasized in a discussion of the critical } \\
\text { role that these play in the work Senhora da Graça. }\end{array}$ \\
\hline $\begin{array}{l}\text { Epigenetics as an Aesthetic } \\
\text { Instrument in a Generative } \\
\text { Virtual Ecosystem [21]. }\end{array}$ & $\begin{array}{l}\text { This paper provides a longer description of the work Senhora da } \\
\text { Graça, the first of the case-studies discussed in chapter 5. It } \\
\text { emphasizes the use of representations from biological processes } \\
\text { and themes, and its free adaptation for artistic practices in the } \\
\text { computational medium. }\end{array}$ \\
\hline $\begin{array}{l}\text { Virtual Worlds as Art Practice: } \\
\text { EvoArt Methodologies [22] }\end{array}$ & $\begin{array}{l}\text { The representational aspects and materiality of virtual worlds are } \\
\text { emphasized in their relevance and potential for contemporary } \\
\text { artistic discourses. }\end{array}$ \\
\hline $\begin{array}{l}\text { Generative Narrative in } \\
\text { Computational Ecosystems [17] }\end{array}$ & $\begin{array}{l}\text { A paper discussing the idea of the CE being instrumentalized as } \\
\text { generative technique for spontaneity and heterogeneity. This } \\
\text { paper discusses some of the ideas presented in chapter } 5 \text {. }\end{array}$ \\
\hline $\begin{array}{l}\text { Generative choreography: } \\
\text { Animating in Real Time } \\
\text { Dancing Avatars [23] }\end{array}$ & $\begin{array}{l}\text { This paper describes the CE being used to animate dancing } \\
\text { avatars. It presents the second of the case studies discussed in } \\
\text { Chapter 5, the choreography for virtual avatars: Vishnu's Dance } \\
\text { of Life and Death. }\end{array}$ \\
\hline $\begin{array}{c}\text { Real-Time behavioural } \\
\text { Animation of Humanoid } \\
\text { Non-Player Characters with a } \\
\text { Computational Ecosystem [25] }\end{array}$ & $\begin{array}{l}\text { This long-paper describes the } \mathrm{CE} \text { as a generative engine for the } \\
\text { animation of a population of gregarious humanoids in the virtual } \\
\text { world WisLM. This paper discusses aspects that are also covered } \\
\text { in chapter } 8 \text {. } \\
\text { A descriptive video was short-listed for best video at the } \\
\text { conference. }\end{array}$ \\
\hline $\begin{array}{l}\text { An Ecosystem Based Model for } \\
\text { Real-Time Generative } \\
\text { Animation of Humanoid } \\
\text { Non-Player Characters } \\
\text { [24] }\end{array}$ & $\begin{array}{l}\text { Given the nationality of the sponsors of this research, and mine, } \\
\text { I found it useful to present this research at the Portuguese } \\
\text { Conference on Artificial Intelligence. This paper is identical to } \\
\text { the previous as it describes the CE as a generative engine for the } \\
\text { animation of a population of gregarious humanoids in the virtual } \\
\text { world WisLM. }\end{array}$ \\
\hline
\end{tabular}




\begin{tabular}{|c|c|}
\hline \multicolumn{2}{|c|}{ JOURNAL ARTICLES } \\
\hline \hline Where is Lourenço Marques?, a & A description of the motivations for the work WisLM is offered. \\
Mosaic of Voices in a Virtual & This forms the base for chapter 7. \\
World [16] & \\
\hline
\end{tabular}

\begin{tabular}{|c|c|}
\hline \multicolumn{2}{|c|}{ WORKSHOPS } \\
\hline \hline $\begin{array}{c}\text { Mapping and Reshaping of } \\
\text { Media }\end{array}$ & $\begin{array}{c}\text { I co-organized this workshop, which was part of the conference, } \\
\text { ISEA 2012. This workshop consisted of two parts: the first was } \\
\text { a preliminary walk through the city of Istanbul in order to collect } \\
\text { relevant data. This data was later used to build digital } \\
\text { interpretations of the subjective experiences of a walk in the city, } \\
\text { using the game platform Unity 3d. }\end{array}$ \\
\hline
\end{tabular}

\begin{tabular}{|c|c|c|}
\hline \multicolumn{3}{|c|}{ SCHOLARSHIPS, AwARDS } \\
\hline \hline Fee Waiver & Goldsmiths, University of London & 2009 \\
\hline $\begin{array}{c}\text { Incentives for Ibero-American } \\
\text { Production }\end{array}$ & $\begin{array}{c}\text { VIDA 12, organized by Fundación Telefónica, } \\
\text { Spain }\end{array}$ & 2010 \\
\hline PhD Studentship & Fundação para a Ciência e Tecnologia, Portugal & $2010-2013$ \\
\hline
\end{tabular}




\section{From Animats to Crowds}

\section{A Literature Review on the Art of Computational Ecosystems}

'When building engaging virtual worlds, a key challenge is to keep them from looking like deserted 'ghost towns.' We want game worlds to be busy, complex and full of life, like a city bustling with pedestrians and vehicle traffic. Alternately a game might call for throngs of people at a fair or party, animals in a lush ecosystem or armies on a battlefield. We want our virtual worlds to be inhabited by thousands of autonomous characters (also known as non-player characters, NPCs). They must have plausible reactions to their environment and to other characters they encounter. When groups of characters meet, we expect them to interact, say by coordinating their motion, or by participating in other kinds of social interactions'.

Jeffrey Ventrella in Big Fast Crowds on PS3

Stuart Wilson, early in 1985, described the Animat model [253], coining a term which would be largely used in the Artificial Life community to address artificial animals with adaptive behaviour. This definition is built on four basic characteristics extrapolated from animals in nature: a) in the 'sea' of sensory stimuli, only some signals are significant; b) animats are capable of actions which tend to change these signals; c) certain signals, or their absence, have a special status; d) the animat act as to approximate and optimize the rate of occurrence of the special signals. The basics for a sensory-motor are described on the first two premises and the latest two bring the notion of needs and the satisfaction of these needs to the model. The development of communities formed by such agents organized in ecosystems is a practice with some established tradition in the artificial life (ALife) community [47, 80]. As mentioned earlier, Computational Ecosystems (CEs) are systems running on computers simulating interactions of individuals within real ecosystems (Figures 2.1). In Oliver Bown, we can read that a typical ecosystem model 'consists of an environment containing resources (or 
also environmental conditions), a population of evolvable agents, and a set of rules defining the relationship between the resource and the agents' [51].

This chapter delineates a context from the thematic tangents that contribute and define this multidisciplinary territory. This is to identify strengths and pitfalls of this practice, in particular when this entails the use of these systems within an artistic context.

For over 20 years, artists have been experimenting with ways in which CEs, as tools and aesthetic frameworks, can expand and enhance their work. The collaboration between artists and scientists within the domain of ALife has produced new art forms, new visual languages, new ways of relating life processes to aesthetics. As new forms emerge, artists are finding even more creative, exciting applications. These are presented in a diversity of forms: from single-channel videos screened on a gallery monitor or video installations, to the intimacy of the personal-computer. Challenging traditional ideas of art and science, these artists use the technology as moving canvases and sculptures for often fantastic, sometimes self-indulgent, but usually powerful art works. They expand the visual vocabulary and force viewers to think about the relationship between art and science in a new way. Understandably, critics and art lovers are confused: is it science, education or is it mere formal experimentation?

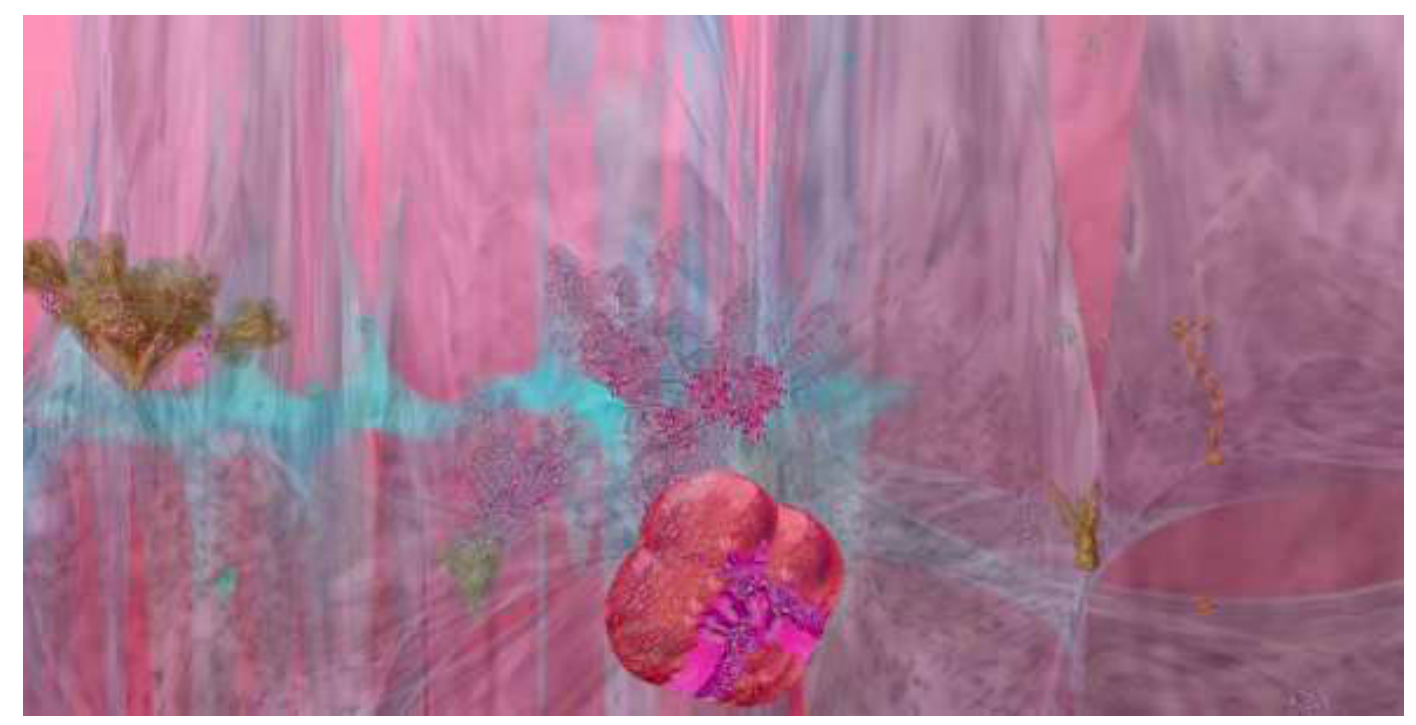

Figure 2.1: Still image from $x T N Z$, an ecosystem of evolutionary creatures whose physical features (shape and sounds) evolve in a generational time scale by means of a software architecture inspired by Mendelian genetics and Darwinian natural selection [19]. 


\subsection{Computational ecosystems}

\subsubsection{The pioneers}

To draw a definitive teleology for these systems we would have to go back to the 1940s, to von Neumman's plans for the universal constructor, a self-reproducing machine, and the early experiments of Nils Barricceli on self-reproducing programs. Or perhaps we should travel even earlier to the fascination of the European aristocracy with automata during the Enlightenment [152], or even much earlier to the Greek broad use of automata of all kinds in religious ceremonies and theater productions [114]. However, for this theoretical framework I will depart from Core War, arguably the definitive technological breakthrough moment which took things to change to the way as we know them today.

Core War was developed by A. K. Dewdney in 1988 to emulate, in a safe environment, individual programs inspired by computer viruses and written in Red Code, an assembly-like language $[75,247]$. In the original version, two programs would compete, attempting to survive the longest in a simulated array of memory. They would perform operations, such as moving position or replicating, in order to force the other program to execute an invalid operation [28]. This game was popular amongst computer scientists and in particular the pioneering community of ALife. The winning strategy ended up being one of building self-replicating programs [8]. One year after, in 1989, this system was used by Steen Rasmussen as the basis to program Coreworlds [199]. Stable structures would evolve from a 'pre-biotic soup' of instructions when processed by a rule processor. Yet, the copy command was flawed and once in a while it would write a random instruction instead of the one intended. This flawed command would introduce mutations into the system and thus the potential for evolution. However, as Chris Adami reminds us 'Rasmussen's experiments in evolution were not successful because his choice of world led to very fragile programs: while trying to copy themselves they inevitably overwrote adjacent programs, such that populations died very frequently' [8].

But, encouraged by these ideas, Tom Ray, a biologist, and a frequent presence in the early conferences of ALife, developed the now famous system, Tierra [202, 201]. Ray's system was also a community of programs inhabiting a bounded section of computer memory. Each program consisted similarly of segments of instructions competing to maximize their 'energy' in the form of CPU time. The aim of the programs might be described as improving the copy efficiency by minimizing their energy expenditure. Individuals must allocate memory before writing in it. Death occurs when the memory fills up, at which point the oldest programs are removed to make room for the new ones. This might in some cases involve writing new instructions into daughter cells. 
Arguably, what makes Tierra such an important instance was Ray's observation of emergent behaviours not initially expected, such as parasitism, when some of the programs started using sections of code from the others to their own advantage ${ }^{1}$.

Computer-programs were exhibiting behaviours that could be described as life-like. More importantly, these life-like behaviours had emergent macroscopic effects not intentionally implemented on the microscopic level.

This was a time of great activity, and a short time later, Karl Sims, a researcher at the Thinking Machines Corporation, caused a sensation with a series of videos of his research on adaptive processes of behaviour. In these experiments, articulated cuboid creatures would compete in a environment simulating physical forces in 1 to 1 tournaments to capture a 'flag' $[220,222]$. The worst performing individuals were removed from the pool and the winners were allowed to procreate a new generation of competitors. Excitement came as the strategies used by the 'creatures' to capture the 'flag' would evolve and improve in efficiency on a generational scale of time.

Around the same period, Larry Yager also observed different strategies for survival emerging in the community of virtual inhabitants in Polyworld [257]. This work offered new paradigms in a diverse number of ways. Contrary to the previous, this was a visual system where a population of multiple individual geometries would roam autonomously in a threedimensional space. As with Sim's cuboid creatures, the individuals had to interpret the world using a perceptual system emulating a simplified system of vision. They would have to distinguish between other individuals and units of food, which would appear dispersed in the environment. Again, as in Ray's work, differentiated strategies for survival emerged in the community. As Yager describes it:

'...one group seems on the edge of psychosis - the "frenetiques", who, zipping compulsively through the landscape, constantly desire food and sex and expend energy on little else. Then there is the "cannibal cult," members of which seek their own to mate with, fight with, and eat. They form grotesque clumps from which they need not move in order to fulfill any of those needs. A third species is the "edge runner." Owing to a peculiarity in the landscape - unlike our own spherical planet, Polyworld can be programmed to have a distinct end of the world - there is a benefit in lurking on the grim of oblivion. Once a respectable number

\footnotetext{
${ }^{1}$ Tim Taylor is a critic of this 'emergent' quality of the behaviour in Tierra. He argues that on the contrary, these behaviours are possibilities already pre-inscribed on the original code [232]. Because Ray forgot to limit the operation of addressing instructions on the virtual CPU to the boundaries assigned to each individual, the 'emergence' observed is more an exploration of existing possibilities left open by the programmer.
} 
of fellow creatures adopt this behaviour, there will always be an ample supply of conjugal partners, as well as old carcasses now turned to food (cited in [143])'.

These early developments have lead to a broad interest in this and other forms of artificial life, and the community of creators has soon expanded. As an example of this growth, the two major conferences specific to artificial life, ECAL and ALife, were in 2013 on their 12th and 13th editions, respectively. Ecology also adopted these systems (known as Agent-BasedModels in the field [197]) as models of the study of communities of organic individuals.

\subsubsection{Evolution as art}

The focus of the first part of this chapter is the artistic production which draws on these systems. The heyday of this practice took place during the mid 1990s, when a series of artists engaged with the possibilities offered by artificial life to explore the social and ethical issues triggered by bio-genetic sciences. This was a topic becoming increasingly popular, triggering simultaneous fascination and distopian fears. Recall that in the beginning of the last decade of the 20th century, the Human Genome Project was launched [3]. This was a time that was culturally characterized by the worldwide expansion of the internet, with magazines such as Wired [2] or Mondo 2000 [212] occupying a central role with agendas strongly engaged with digital technologies and a fascination with genetic engineering.

\section{The Iterative Genetic algorithm}

Earlier, in 1986, Richard Dawkins published The Blind Watchmaker, which in order to illustrate evolution by natural selection, described a groundbreaking software. This would generate 'biomorphs', symetric bi-dimensional stick-figures described by genome-like structures. A human observer/operator would pick two individuals from a pool of biomorphs, to reproduce these and generate a new breed. Despite the fact that Dawkins himself was a biologist, the process he described would become a fundamental process for Evolutionary art known as the IGA (Interactive Genetic Algorithms) ${ }^{2}$. It was in this context that William Latham, in the early 1990s, created his trademark organic-like 3d shapes. Latham, trained as an artist in the Royal College of Art in London, developed the Mutator interface in partnership with the programmer Stephen Todd at IBM [237, 238]. The Mutator would make use of a similar methodology to Dawkins to run evolutionary processes, creating selective pressure in order to drive the evolution of $3 \mathrm{~d}$ shapes, which were described by genome-like blueprints. Other pioneers of

\footnotetext{
${ }^{2}$ In Computer Science, the term Interactive Genetic Algorithms has a wider use as it describes methods from Evolutionary Computing, namely the Genetic Algorithm, using human evaluation as part of the methodology.
} 
Evolutionary Art, such as Steven Rooke or Jeffrey Ventrella, also use this methodology to produce static imagery $[211,251]$. Similar methodologies are followed in other creative areas, such as Design and Architecture.

Mitchell Whitelaw makes a good characterization of this practice in the book Metacreations [251], where he examines this praxis as a cultural form. The proceedings of the EvoMusArt workshops and conference, and the books The Art of Evolutionary Evolution from Romero et al. [210], and An Introduction to Creative Evolutionary Systems from Bentley et al. [36] are collections of articles and papers providing also providing good resources for this practice by overviewing the field.

\section{Dynamic Genetic algorithms}

In the mid 1990s, a new generation of artists built the grounds of their practice upon this process. If these first pioneers of Evolutionary art worked like gardeners breeding species of plants by their selective action ${ }^{3}$, this second generation practised an art of constructing artistic digital ecologies, 'Cybernatures' as Whitelaw would call them. Amongst these are Technosphere [192] and A-Volve [225], from Jane Prophet and Gordon Selley, and from the pair Christa Sommerer and Laurence Mignonneau, respectively, which have built on the anterior practices of the pioneers to expand these. As with Latham, the space and creatures are Cartesian and individuals are described by genetic blueprints. However, as with the pioneering systems, such as Tierra and Polyworld, these works offer communities of individuals interacting in virtual environments while competing to perpetuate their genes. In contrast with Latham's IGA procedure, where a human is responsible for the selective evolutionary force, the evolutionary pressure was determined here by the evolved strategies of survival and the contingencies of the encounters in a crowded environment.

Additionally, in both A-Volve and Technosphere, the internet was used to allow a remote audience to feed the system with new individuals. These authors brought their works to a wider audience. Later, the virtual creatures would additionally 'send' emails to their creators. Other works in the same period followed similar reasoning, building hybrids that expanded the realm of CEs from the computer to systems where the user/audience also became connected parts $[173,228,124,69]$.

\footnotetext{
${ }^{3}$ This analogy was used by Latham to describe his own work. Cited in page 56 of Whitelaw's Metacreation [251].
} 


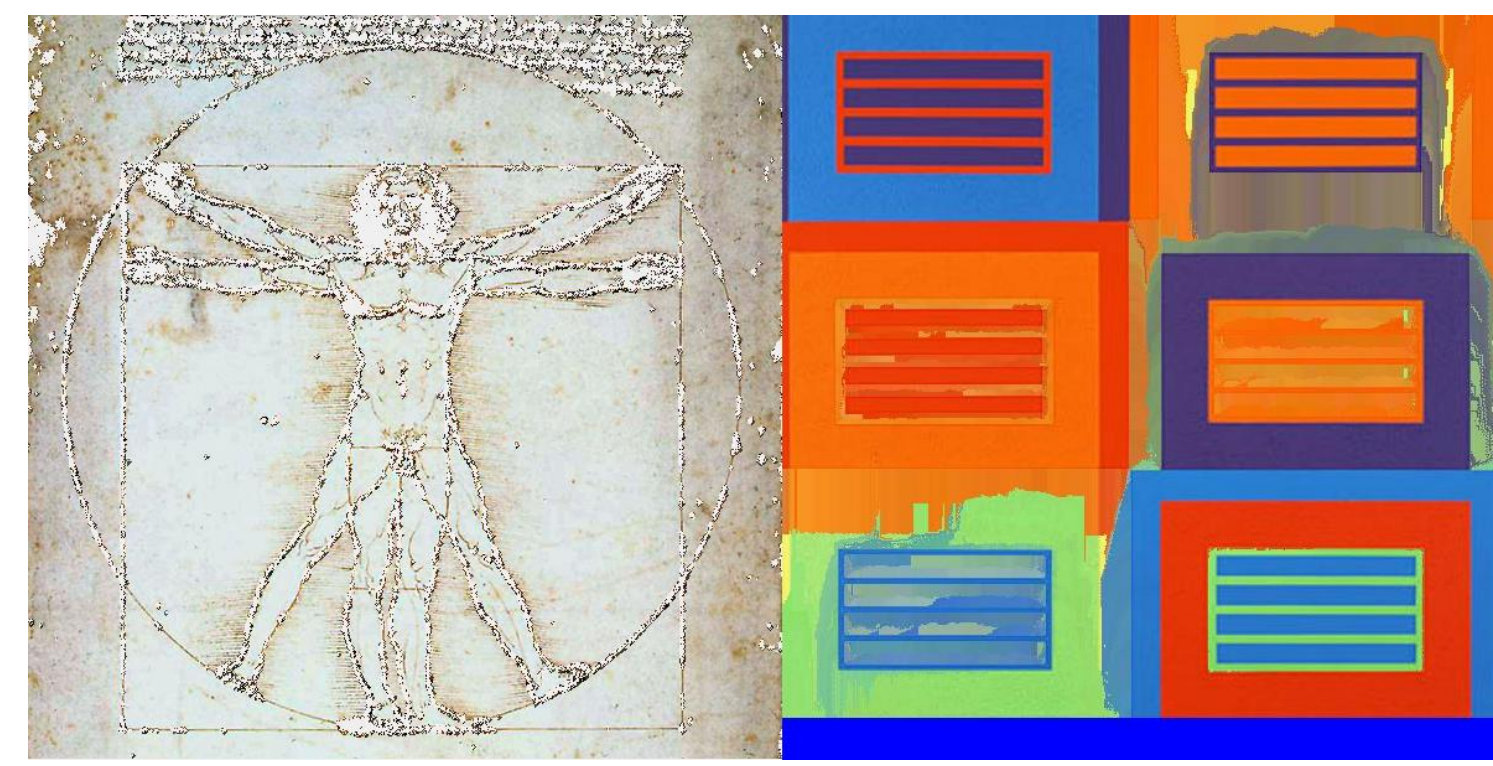

Figure 2.2: Left: A reinterpretation of the famous work of Leonardo DaVinci the Vitruvian Man, using a CE. A community of dynamic and 'starving' pixels eat sections of a digital representation of the drawing based on the hue value of the other static pixels, which become resources. Right: A similar process is at the origin of the Halley series, a series of dynamic works where the digital representations of the paintings from Peter Halley appear reinterpreted by means of the CE.

\subsubsection{The artistic practice using CEs}

As an artistic technology, the art using CEs does not appear isolated in time; rather it utilizes established pictorial and representational traditions. It inherits methods and canons, which have been in practice for centuries and now manifest in structuring these new works. For instance, the canvas is slowly and patiently filled with 'virtual ink' in Annunziato's compositions of curved filaments $[14,15]$. Each agent in the canvas is a virtual drawing brush, which adds virtual ink to the blank canvas. It keeps doing so until it reaches another agent; then it stops the activity and dies. Annunziato's methodology resonates the processes involved in classic drawing and painting: layers of ink are added to the canvas in a material composition of juxtapositions and accumulation. Later, McCormack and Burnhoff picked from Annunziato's work to build on the levels of freedom and the variety of brushes used [48, 53]. A similar procedure is followed in Driessens and Verstappens's works when the canvas becomes an archeology of spatial changes [245]. These works portrait the spatial dynamics of the community of agents working together in the canvas exhibited at the gallery. Drawings result from changes in concentration and density in the community. However, in an interactive process, visitors can destroy whole populations whose drawings they do not like. With the help of a 
touch screen, they can choose the new direction of the work from a set of possible and logical continuations, which result from the present configuration. In a process of subtraction, similar to the one when a material block is carved to let a sculpture emerge, Driessens and Verstappen's audience removes raw possibilities from a block of virtual possibles to let the work progress in the selected direction.

These operative arithmetics of addition and subtraction form the essential dynamics of this 'vivid painting in motion' as Lioret refers to in his works [146]. Other classic representational strategies invoked in the new art form are the omnipresent notions of interior and exterior. This echoes the classic idea of man standing outside of reality to observe it. And this stance is reflected in the classic arts by the frame and the sculpture pedestal. This duality, where works appear framed in a 'window', transverses almost all the instances analyzed (Appendix-A). This 'hypermediacy' is greatly justified by the computational nature of the artefacts and their windows paradigm. This duality is used and integrated with contextual advantage in the diegesis of works such as Senhora da Graça [21] or EIDEA [173]. These examples integrate the duality of interior/exterior to approach an environmental thematic and emphasize the contrast between the natural environment outside and the machinic artefact. However greatly influenced by classic art, artists making 'vivid painting in motion' do not constrain their practice to established processes and methods. The artefacts produced owe much to contemporary art forms, such as video and installation art. Challenging the dichotomy of interior/exterior, works such as Artificial Natures [248], Pandemic [83], and Eden [158], acknowledge this difference and try to integrate the audience phenomenologically in the virtual and tangible spaces. These works combine the $\mathrm{CE}$ with sophisticated techniques of computer vision to capture the audience's location in a subtle way. For instance, the physical presence of the audience in Eden energizes the virtual world. The audience becomes the center of attention of the virtual creatures which sing to call their attention and attract them in order to get more energy. A similar approach was followed in the Artificial Nature series. The body's shape and volumetric information is captured and transformed into energetic particles in the virtual space. On the contrary, in Pandemic the avatars of the members of the audience become a spreading disease.

As mentioned earlier, the generative processes of the CE rely on the gradual and cumulative effects of changes produced by the dynamics of the autonomous elementary units of the system. Time is omnipresent and an essential component for the CEs operation. It is structuring, and works produced using CEs are naturally affiliated with the traditions of kinetic art. These tend to be abstract in their appearance. Individual representation varies and individual members of the population are represented by dots (e.g [245]), or lines (e.g [14]), or shapes (e.g [77]), or three-dimensional volumes (e.g [21]). The data provided in Appendix-A illus- 
trates the openness of the methodology, and none of these forms dominate the others. In some instances, we have outcomes of visuals rendered with plasma-like qualities [245], whereas in others we have communities of $3 \mathrm{~d}$ avatars walking in virtual worlds [16]. Yet only in marginal cases, the works represent the appearance of existing life-forms. Abstraction is dominant and the motto, life-as-it-could-be, inherited from ALife reigns all over the spectrum of this practice (Appendix-A).

The 'technical support' and plasticity of CEs However, despite the dominant outcome with abstract visuals and sounds, CEs are still highly representational forms. This was clearly discussed by Mitchell Whitelaw [251]. ALife art owes much to the tradition of Organicism in the arts, with its agenda and interest on representations of life. It is suggested that ALife art is not representational in the appearance of life forms, but rather in the way life operates. And this is the fundamental aspect that transverses the heterogenous artefacts (results of the survey are presented in Appendix-A), with all their differences and disparate outcomes: a set of structuring ideas and a technology. Here it might be helpful to invoke Rosalind Krauss when she questions the modern medium-specificity in the arts. In Under the Blue Cup, she argues that the medium is not reducible to the 'specific material support for a traditional aesthetic genre' [135]. This expanded notion of the medium that she is proposing, detached from the technical substratum, is rather grounded on a set of historical situated practices, or what she calls the 'technical support'. The technical support recuperates more than a century of modernist practice restoring autonomy and extending the life of the white cube of the gallery. It is an underlying field that supports the work of art as canvas supported the oil paint. For instance, in Ed Ruscha, the automobile is the technical support for the subordinated paintings on parking lots and gas stations ${ }^{4}$; in Sophie Calle, it is photojournalism that supports the gallery work $[135]^{5}$. ALife provides an example of a contemporary art genre where it is not the technological medium, or more precisely the set of techniques, what ultimately constitutes or defines the ethics/aesthetics. The technical support of ALife art are the ideas and methods informing this particular artistic genre.

By the same token, a similar reasoning can be suggested for Evolutionary art. Works on

\footnotetext{
${ }^{4}$ Ruscha is commonly associated with the Pop Art movement. His work manifests a clear interest in Los Angeles' subculture, which is central to the themes and styles of much of his paintings, drawings and books. Famous works include sentences using the vernacular language from Los Angeles overimposed on Southern Californian landscapes or on visuals with particular references to the movie industry, such as the Paramount mountains.

${ }^{5}$ Calle's work is recognized for its journalistic-like investigations. The artist explores identity and intimacy with a detective ability to observe (and register photographicaly) the behaviour and actions of strangers, to construct their identities by following them and investigating their private lives.
} 
Evolutionary Art, from which CEs are the newest forms, are aggregated by a shared basis of a number of techniques and common ideas. We can then freely say that the technical support of EvoArt are the ideas about evolution by Natural Selection and Mendelian genetics that inform this practice. What follows from this assumption is that the CE may then be reduced to a generative technique, part of the set of methods from Evolutionary Art and ALife. As a generative technology, removed from its technical support, the $\mathrm{CE}$ is open and may be used within diverse agendas and in a wider spectrum of displays and modes of exhibition. The critical aspect of this technique lies on this plasticity. This technique does not get extinguished in the variety of modes of display. The essence is that of a generative method, and this framework provides for the production of a diverse number of themes and outcomes. These can include a wide spectrum of opportunities: abstract 'vivid painting in motion' [146]; as well as food chains with NPCs, composed of herbivores and carnivores acting autonomously and roaming in the virtual space [192]; or, as it will be argued here, the animation of performative dancing avatars in virtual environments [23]; or even talkative gregarious humanoid avatars in virtual worlds [16].

\subsubsection{The new paradigm provided by sonic ecosystems}

I will build this argument from the practice of Evo-artists in the recent years who have used the CEs to explore audio possibilities taking advantage of the inherent dynamism of the systems. Making use of a similar methodology to the one offered by pioneers exploring still imagery, one approach has been to sonify the genomes. Artificial Natures, Plague, Listening Sky and Feeping Creatures offer examples of such an approach [39, 78, 77, 248], transcribing each elementary unit contained in the blueprint into an audible sound.

Another take on this subject was explored more recently by Eldridge and Dorin [90]. This consists of sonifying the dynamics of the community to make audible the behaviour and characteristics of the individuals. In Eldridge's work, the energy level and the spacial disposition of the individual are decoded into different scales of frequency in the audio compositions. In Plague and Spinners [39, 78, 77], Dorin and Berry went a step further and sonified some of the ephemeral states occurring in the lifetime of the individuals, such as when they are born, or reach maturity. In Dorin's words, 'these incidents are interpreted sonically in the current system as discrete, audible events of different manually constructed timbres' [79].

I argue that the above challenges the established paradigms of EvoArt. The pioneers have looked straight at the procedure entailed in the Genetic Algorithm from Evolutionary Computing as a working methodology that we can call the Gtype-Ptype paradigm. The genome (Gtype) forms a set of syntactic elements, which are then translated into semantic formula- 
tions (Ptype). As mentioned earlier (while formulating the hypothesis in chapter 1), in the case of Latham, the genome was decoded as a three-dimensional shape using a morphological grammar [237, 238]; in Sims or Rooke, the Gtype is transformed in complex abstract imagery using a set of mathematical operations [221, 251]. Initial works on CEs, such as Tierra [202] or Polyworld [257], or even the later A-Volve [225] or Technosphere [192], have built straight out on this paradigm. However, in the work of Eldridge, Berry and Dorin, the syntactic elements are not only the genomes, but rather the ephemeral behaviours and internal states of the individuals. As Eldridge puts it, 'the resultant sonic behaviour is contingent upon the interacting components and emerges at run-time' [90].

This thesis argues that these sonifications of CEs form a new paradigm in EvoArt. If we build on this last take and consider as syntactic elements the ephemeral actions performed in the context of the ecosystem, we have a powerful generative mechanism which can be easily transferred from the sonic installations to other creative domains. The subjective process of semantic interpretation guarantees the plasticity of the methodology (I will take this argument further in chapter 5). One of these possible applications is argued to be the animation of communities of NPCs in virtual worlds, something that will be discussed further and demonstrated in chapters 5 and 8.

\subsubsection{The 'remediation' of the technology of the CE in Virtual worlds}

The fact that artists are using the natural dynamism resulting from the ephemeral events in the ecosystems provides a new paradigm that challenges the established IGA. This new paradigm might enrich VWs in a process of 'remediation' of the technology of the CE.

VWs are characterized by a constant movement of technological incorporation and expansion. These are composed of a suite of articulated technologies. From the initial text based multiplayer Role-playing-games and chat interfaces, VWs have evolved, growing based on the incorporation of 'new' technologies such as the three-dimensional space, the cinematographic language, the first-person-point of view or, as it is argued here, the CE.

When applied within the realm of VWs, the CE is said to be remediated since, on the one hand, it maintains the features traditionally associated with computational systems emulating animals that co-inhabit a space and reproduce and prey on each other. This was something already demonstrated in Technosphere [192]. On the other hand, in the process of remediation, the 'old' technology gains or creates new social spaces or forms of engagement, new modes of interacting and being. Adapting the paradigm discussed above offered by artists exploring sound, in the context of VWs, the CE can be looked as a generative method for behavioural animation used in the animation of groups of general purpose NPCs. In chapter 8 , I will 
demonstrate this with the work WisLM. In the following section, I will take a look at current standard methods for animating groups of NPCs in the fields of simulation and games.

\subsubsection{Behavioural animation of groups of non-player characters}

Groups and crowds' simulation refers to the modeling of aggregates of agents interacting in a shared space, forming flocks, schools, herds, armies, pedestrians, traffic with vehicles, etc. The intelligence of each individual varies from none to high. Considerable research has been produced in the field [234, 187]. The main areas of application include the simulation of evacuations, as well as video games, movies, and educational applications. Most of the work, however has been in creating virtual humans that walk in a believable manner [187].

The strategies to deal with the problem of group animation in real-time can be classified according to the way individuals are represented and articulated. This involves the dimension of the population to be simulated, the intelligence of the self-animated characters, their control mechanisms and the collision and handling methods.

\section{Rule based approach}

One of the first to apply these principles was Craig Reynolds in his research on flocks of birds and schools of fish [206, 207]. Reynolds uses the term 'boid' to refer to the individual entities. In an elaboration of a system with oriented particles representing boids with specific control rules, a reduced set of rules combines to determine collision detection and avoidance. Each boid, only perceiving its local environment, maintains a proper position and orientation with the rest of the group. This work was recently expanded to the architecture of Playstation 3, simulating a population of 15000 simultaneous individuals [205].

\section{Particle based approach}

In this class of models, individuals are considered as particles subject to physical forces of attraction and repulsion between obstacles and the other individuals in the simulation. These are systems typically composed of many participants with significant dynamics.

Helbings's social force model describes group dynamics based on forces which can be of attraction or repulsion based on the interplay of environmental and social forces [111]. Forces of attraction drive the individuals to their objectives whereas forces of repulsion and tangential forces drive them away from certain specific locations. This allows for realistic 'pushing' behaviours and variable flow rates. This work was extended in Nuria Pelechano et al's system, 
HiDAC, with behaviours inspired by biological emotions, such as panic [185]. The crowd simulation deals, at a higher level, with way-finding processes where individuals acknowledge the structures of a building, as well as an associated low level motion based on social forces. Other approaches, equally relevant, draw their inspiration on magnetic forces and fluid-dynamics to model the flow of pedestrians [110].

Relevant results were achieved with this methodology. For instance, diverse properties observable in human crowds emerge in these systems, such as line formation or patterns of motion. However, because individuals are represented as particles, the flexibility of the methodology is somehow constrained, and the addition of heterogeneity and complex behaviours becomes increasingly difficult to achieve.

\section{Cellular-automata approach}

Cellular automata provide a simple and effective technique to model complex and emergent behaviours. One of the earlier developments was in the simulation of cars in traffic [179]. A checkerboard matrix of cells represents the environment and the road tracks. The state of each cell is determined at discrete time steps when neighbours in the matrix are inspected. In Nagel's work, this description includes the other cars as well as physical obstacles in the road. The floor is discretized, so cars can only move to adjacent cells and two cars cannot be simultaneously in the same cell. The coordination of the global update of states simultaneously act on all the cells, generating the illusion of movement. This illusion is created, for instance, when considering two adjacent cells; the first transitions from the state 'occupied' to the state 'empty' whereas the adjacent cell, initially 'empty', transitions to the state 'occupied'. In a well known phenomenon typical from cellular automata, the sequential application of these local rules to the whole space generates emergent effects and collective behaviours observable in groups. More recent approaches consider that individuals are particles in a grid of cells, and that each of these can only be occupied by one individual. The particles/individuals have rules for movement that equally take into consideration the velocity and number of the occupied neighbouring cells. This technique has been used to simulate evacuation environments [32], or pedestrian dynamics [58], and to manage passenger handling [215].

In Shadschneider et al. [214], the cellular structure is additionally characterized by a predefined level of desire associated with each cell, which, combined with more dynamic effects generated by the passage of other pedestrians, guides the transition of states. Recent developments of this approach introduced the anticipation of the movements from other pedestrians, in particular in counter-flow scenarios [181].

Some of the limitations of this approach are pointed to by Pelechano et al. [185], such as 
the inability for direct contact between the agents. Furthermore, the movements generated are not very realistic, with agents showing some difficulty in turning as a consequence of the checkerboard configuration in crowded situations.

\section{Autonomous agent approach}

Multi-agent systems (MAS) can be described simply as systems of agents interacting in collaboration or competition. These are popular due to their flexibility. Examples of MAS in crowds include Batty, Christian et al and Tomaya et al's work on pedestrian simulations $[34,102,239]$. These share the fact that agents incorporate some of the properties described in the previous categories: forms of attraction and repulsion generated by other individuals and some points of reference in the environment. Musse et al [200] and Shao and Terzopoulus [218] include basic reactive behaviours and a cognitive level. Shao and Terzopoulos draw on a technique inspired in the AI of games known as Influence Maps. These are two-dimensional grids superimposed onto the world. Each cell indicates its own static or dynamic content. For instance, these can specify where is the location of the enemy forces that we need to avoid. Influence maps can be extended as spatial databases, with diverse maps functioning as layers, each layer describing a particular type of data. The agent combines these different maps to generate a final map with the degrees of desirability for each cell [32]. In Shao and Terzopoulus, the actions of the agents are not limited to the movements but also introduce some interactions between the agents as well as between the agents and the environment, such as 'buy a ticket', 'sit and watch the play', etc. Musse et al's ViCrowd [178, 235] generates crowds based on group properties rather than individuals. These have knowledge about the world and are equipped with a system of beliefs, desires and intentions in the form of goals, internal status and information about the world. Qiu and $\mathrm{Hu}$ also developed an agent-based framework, which emphasizes the importance of groups in crowds [196]. This generates crowds of different dimensions, which take into account the different influences of navigating inside one's own group and between other groups.

\subsubsection{The problem of the persistence of virtual living populations}

So far, all these previous approaches seem to have a predefined density of population. The $\mathrm{CE}$, however, offers a different intrinsic problem since numbers fluctuate when individuals multiply or destroy each other. The problem of what is called the persistence of these systems arose in the first of the developments of the practical component of this research (Senhora da Graça) [21]. The issue was maintaining a living population without going extinct or becoming 
overpopulated. With a frustrating frequency, the population in the system got erased by the natural course of the individual interactions.

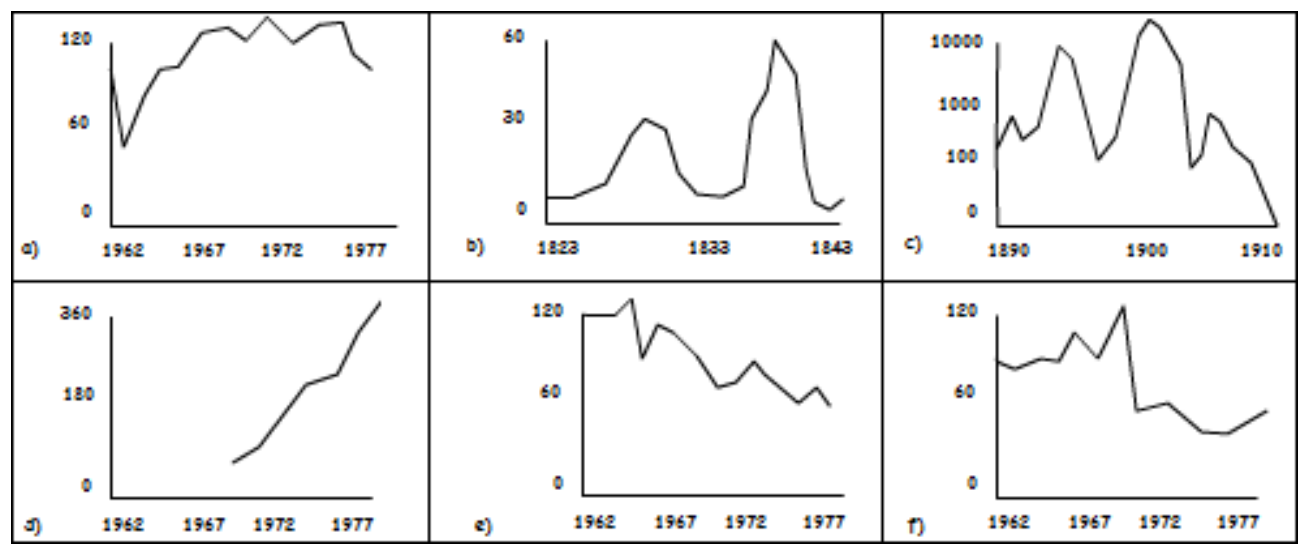

Figure 2.3: Some examples of population changes in natural ecosystems. a) Song Thrush in English Farmlands, b) Lynx in the Canadian Artic, c) Bupulus, a moth in German forests, d) Collared Dove in England, e) Spotted Flycatcher in English farmlands, and f) Whitethroat in English farmlands. Scales: the abscissa in all cases is in years. The units of density are relative ones in (a), (d), (e) and (f), and are set to 100 in either 1966 (a), (e) and (f) or in 1972, (d). In (b) the units are thousands trapped per year and in (c) numbers per hectare of forest floor. Source: [190]

The nature of population dynamics is for populations to be stable or tend to be. Paraphrasing Stuart Pimm, a biologist and theoretical ecologist renown for his work on biodiversity and conservation, 'natural populations are characterized by patterns of change that keep their numbers within bounds... following a perturbation, densities tend to return to a recognizable equilibrium level, or more rarely, a cyclical pattern'. However, he continues 'a minority of populations fluctuate so wildly that an equilibrium is not obvious' [190]. Since population numbers may fluctuate 'so wildly' in nature, why should this be different in virtual communities? Tom Barbalet, in Biota pod-casts referring to his project Noble Ape, commented on the rapid decay of the population and the consequent lack of interest from users [33]. Bedrich Benes, on the contrary, faced the inverse problem in his ecosystems of flowers and identified overpopulation as one aspect to be tackled during the development of systems [35]. However, if these oscillations occur in an uncontrolled way in a Virtual World, they put the entire model at risk by either compromising the computer power or the network latency in a networked environment. This can occur when: i) the model gets overpopulated, or ii) the extinction of a keystone species drives the population to a cascade extinction ${ }^{6}$. Gordon Selley, who co-

\footnotetext{
${ }^{6}$ In Ebenman and Jonsson [88], we can read that a keystone species is one 'whose loss is likely to have serious effects on the continued existence of other species and, hence, on the long-term persistence of the ecosystem.'
} 
authored Technosphere with Jane Prophet, recalls a situation where the audience created too many carnivores who ate all the herbivores, and consequently the authors had to repopulate the world [194].

The persistence of CEs is an open-problem with no standards yet established. Some modelers use a fixed number of individuals [202], others let the numbers of inhabitants fluctuate between two thresholds [257], while others enforce the system to be explicitly homeostatic [35].

In terms of the type of model of population, there is also no consensual development. If some use a single-niche with homogeneous populations (with none [202] or one single type of external resource [257]), others build systems where at least three different trophic levels appear clearly differentiated [192], typically with populations of plants, herbivores and carnivores (Appendix-A Table 6.1 shows some of this diversity).

In chapter 6, this problem, known in conservation biology as stochastic extinction in smallpopulations [63], is addressed. Small populations are those potentially offering greater risk when their numbers fluctuate. For instance, it doesn't take a large number of consecutive deaths to significantly lower the density in a small community. The aim, however, is to systematize different strategies to cope with the problem of extinctions when trophic interactions emerge from the interaction within a small population (less than 200 individuals ${ }^{7}$ ). For this purpose, different approaches were developed to study this problem (See chapter 6).

\subsubsection{The context of WisLM: Evolutionary Art and Relational Aesthetics}

Some of the immediate advantages of the use of CEs in the animation of NPCs, and of this research in particular, is to offer to EvoArt, on one hand, a framework for the development of persistent communities that tolerate fluctuations in density; and on the other hand, the possibility of engaging with new dialectic territories. In his recent paper for EvoMusArt, one of the leading artists of this movement, Jon McCormack, expresses his frustration with the marginal place that Evolutionary art occupies in the art world. In his words, 'the art world quickly grew tired of evolutionary art only a few years after it began and had little or no interest since' [157]. One of the reasons for this, McCormack suggests, is the lack of articulation between the practice of EvoArt and theoretical art. To this claim, I would add a particular attention to the articulation with contemporary art theory. Discussions on EvoArt take place

\footnotetext{
${ }^{7}$ This was the maximum number of simultaneous NPCs that I was able to handle while maintaining a satisfactory performance and frame-rate, WisLM [18]. For the purpose of the present research, this was considered a reasonable extrapolation.
} 
within a confined territory, in the technological milieu, such as the conference EvoMusArt. With the exception of Metacreations from Mitchell Whitelaw, the epistemology of the genre, such as The Art of Evolutionary Art [210] or An Introduction to Creative Evolutionary Systems [36] or Virtual Worlds [49], focuses to a great extent on technical aspects of the artefacts, and references to contemporary art theory are barely existent and appear secondary to the technical discussion. This thesis is no exception, where once again the resolution of practical problems takes precedence over the aesthetic issues. However, it can be said that this type of material and processual attention reinstates pre-modern practices with an exacerbated attention to the physical artefact. This fetishisation of software and code owes greatly to the realm of software art where artists reinstate modern precepts and are interested in showing the code and to exhibit the processes of construction. As mentioned earlier, the technical support that Krauss is referring to is a set of ideas informing and unifying a practice, not so much the technical implementation of these ideas.

The words of McCormack are echoed here. With WisLM, a tangential route is pursued by invoking Relational Aesthetics to the center of discourse and practice. Relational aesthetics is a contemporary art theory, which some argue is the new emergent 'ism' of the arts. Nicolas Borriaud advocates that in the last two decades there has been a clear pattern of artistic practices where humans become the center of attention. In his view, people and people's stories form the core of the contemporary practice. Drawing along similar lines, Claire Bishop in turn emphasizes a paradigm of participation where works become the means to promote human encounters [41]. The artwork, WisLM, precisely juxtaposes these ideas with the aim of bringing these topics to the core of the practice of EvoArt. This work and its motivations will be discussed in chapter 7. For this project, the animation of NPCs by means of the technology of CEs is a critical part of the process. This implementation is discussed in chapter 8 .

\subsubsection{Final notes}

This literature review emphasizes a movement transitioning the methodological processes informing the artistic use of the $\mathrm{CE}$ from the initial explorations much focused on the IGA paradigm to a greater attention being payed to the internal dynamics of the ecosystem. This more recent approach has been expressed when artists sonify the dynamism of the functioning activity in the ecosystem. This is in the form of transforming into audible sounds the individual characteristics and behaviours of the population during their normal activity.

Throughout this thesis, the $\mathrm{CE}$ is suggested as a generative animation tool. It is argued that the CE provides a novel operative framework for the animation of general purpose NPCs. This draws on work from Evo-artists using CEs to produce granular soundscapes. The internal 
dynamics of a living ecosystem, the spatial movements and the internal states of the individuals in the population provide for a tool-box that can be described and used as a generative method for heterogenous and spontaneous behaviours.

The final part of the chapter looks at the other standards currently used in the field of behavioural animation of groups of NPCs. Existing mechanisms for generating micro-behaviours in crowds, such as groups of conversational humanoids, are in general purpose-built and articulate with mechanisms of macro-behaviour controlling the movement. The CE combines these micro and macro behaviours into a single model.

However, on using CEs we face some critical problems. One of these is the fact that populations fluctuate, and some of these fluctuations might be too wild, thus forcing the systems to collapse. In order to easily and confidently implement CEs that can be exhibited continuously for long periods of time to the public an investigation is suggested. This is on a system that automatically adjusts its own parameters towards its persistence.

Finally, new utilitarian and discursive territories are suggested as emerging from this study. Some authors are critical about the self-contained dialectic domain of EvoArt. Here, it is suggested that the instrumentalization of their nature, as an abstract and general purpose animation tool, endows an expanded use as narrative-producers. This prospect is argued as to widen the realm of EvoArt, in a conceptual movement from an interest in 'interaction' towards 'participation' and looking at these systems as story-producers. 


\section{The Materiality of Evolutionary Art}

\section{Artificial Life Models in Software}

\subsection{Introduction}

This research is about VWs populated by virtual life forms, what Bruce Damer calls biots [68] and are usually described as animats [254]. The objective of this section is to look at the tradition of building synthetic life and ecosystems in the field of artificial intelligence, in particular to examine CEs from the perspective of the materiality of these artefacts, to scrutinize methods and techniques involved in their construction. For this purpose, a contextual review of the fields where this technology originated, AI and ALife, is produced. A systematization of the techniques which constitute the technological substratum for the construction of CEs is developed. I will identify and describe each of the 3 main paradigms that inform the development of CEs: a) The Gtype-Ptype, b) the cellular automaton; and c) the co-evolution in multi-agent-systems. These appear in this document in arbitrary order, not by chronology of events but in what seemed to be a logical continuity in terms of functionality.

\subsection{Biots and digital aquaria of life: Al techniques informing the construction of computational ecosystems}

Subsidiary to computing, developments in the history of ALife appear intertwined with developments in this science. When we trace back to the early primeval times of computing, we can start to unveil some of the main roots of the paradigms informing today's practice of virtual communities. 
The years of 1953 and 1954 are critical for ALife historians. This was when Esempi Numerici di Processi di Evoluzione was first published, one of the earliest records of an evolutionary simulation, of what is now called artificial life. The author was Nils Barricelli and this work was published in the journal, Methodos, in 1954 [94]. In 1953, Barricelli was visiting Princeton University, then a leader in the new field of computing mainly due to the presence of John von Neumann and his development of the MANIAC machines. During the cold war, von Neumann was a principal member of the Manhattan Project, involved in the development of the hydrogen-bomb. This granted him the means to build a new type of computer: a 'storedprogram' machine that could be reconfigured quickly to perform different tasks by changing its software rather than rewiring its hardware. Von Neumann himself had an interest in self replicating machines and, earlier in 1940, designed the theory for the Universal Constructor, the plan for a self-replicating machine in a cellular automaton (CA) environment (CAs are explained in detail in the following sections). By 1953, his machine was simulating nuclear explosions by day and modeling the evolution of artificial life forms, the creations of Nils Barricelli, by night [87].

In an extraordinary coincidence, in the same year of Barricelli's early experiments, James Watson and Francis Crick uncovered the digital foundations of the structure of DNA, which greatly inspires the first of the paradigms informing the construction of CEs.

\subsubsection{The first class: Gtype-Ptype}

Inspired by vertical genetics, the theory that parents' phenotypic traits are passed to their offspring through their genetic information, Evolutionary Computing (EC) developed a crucial methodology that can be described as the Gtype-Ptype paradigm. The Gtype or encoded description of the object (the genome) is transformed into a phenotype, a representation of the individual (the Ptype) [137].

Since the early days of computing, a number of schools, Genetic Algorithms (GA) [116, 117], Evolutionary Strategies (ES) [203], and Evolutionary Programming (EP) [95], have developed independently of each other, exploring the optimization processes seen in natural selection and adaptation. Later in the 1990s, these aggregated into a common discipline called EC. EC's techniques are methods to optimize the search of large multidimensional solution spaces for optimal results. Overviews are provided by Bentley and DeJong [37, 71] $]^{1}$.

Usually, variables describing one problem and its characterizing features are expressed in the genomes of a population. This genetic representation becomes improved with time, pro-

\footnotetext{
${ }^{1}$ These are approaches mostly focused on optimization. Keneth Stanley followed a divergent trajectory investigating novelty search [230].
} 
gressively closer to the ideal solution that solves the problem. The improvement is caused by a fitness function that is used to evaluate individuals and exert a selective evolutionary pressure. This fitness function usually quantifies the assertiveness of an individual solution for the specified problem. For instance, if the problem in question is a locomotion problem, being described by: moving from point $\mathrm{A}$ to point $\mathrm{B}$, then the closer one individual is to point $\mathrm{B}$ the higher the fitness function will evaluate his performance. With this criterion, the fittest individuals from an offspring can be chosen to procreate while the others are removed. The resulting offspring is usually the product of genetic operators: mutation and crossover. Gene values are mutated, and if there is more than one parent, their genes get mixed. This paradigm explores what is called a fitness landscape, a domain of possible solutions for the problem in analysis. In a multi-peaked landscape, the population tends to aggregate around niches of higher fitness. This process adapts the gene pool of this population to solutions of the specific problem $^{2}$.

\section{Embryonic expression}

In the Blind Watchmaker [70], Richard Dawkins describes Biomorphs, how he has used the methodology described above, for the exploration of the evolution of morphologies. Dawkins, a biologist, was back then interested in showing the mechanisms of natural selection. In this seminal experiment, an initially symmetric stick figure produces an offspring of individuals with genotype distinctions, induced through small mutations which are performed on the parent's genome. Dawkins exerts the fitness pressure himself by selecting the fittest individuals from this offspring. This individual (the fittest) will be the next progenitor, on which a similar operation will be performed in a conceptually endless loop.

Biomorphs showed that a similar seed could indeed be evolved via processes of natural selection and adaptation to a multitude of morphologies (or phenotypes). The only difference is situated in the different driving processes, which accumulate small changes, tracing a different genealogy for each individual through the course of generations.

Artists such as William Latham [237, 238] and Karl Sims [220, 221], used this paradigm to explore the creation of sophisticated imagery. They were the first artists to associate their work with the terminology and concepts of the unfolding process of DNA [251]. The 'embryonic' or 'expression' step (the conversion from Gtype to Ptype) could indeed be whatever the artist wanted. Latham used this paradigm to evolve three-dimensional shapes, which were

\footnotetext{
${ }^{2}$ The above is a description of an approach focused on processes of optimization. Keneth O. Stanley provides another paradigm focused on novelty search. NEAT is a GA for evolving artificial neural networks. It changes the weight and parameters attempting to balance the fitness of the evolved solutions and diversity [230].
} 
transcribed from the genotype with the help of a transcription grammar defining shapes and affine transformations; inspired in Koza's GP [134], Sims bred and evolved Lisp expressions combining mathematics with primitives of image manipulation to synthesize imagery. Other authors followed exploring this methodology $[211,240]$ in a style Whitelaw has christened as Breeding [251]. Lewis describes around 200 references of different works in a multitude of uses, from producing simple imagery to cartoon characters or architectures [144].

\section{The fitness function}

In the Breeding style, the artist who defines the algorithm sets the individual's initial state. Then, when an offspring is generated, the artist as a 'gardener' selects the best phenotypes to use as seeds for the next generation. This process continues endlessly until the 'gardener' is satisfied with the resulting images. The artist drives the computational power of the computer in a shared creative process, where the selective pressure is explicitly exerted from the artist him/herself, a process known as interactive evolutionary computing (ICE) [101], also known as IGA, standing for interactive genetic algorithm.

As Galanter reminds us in his recent paper The Problems of Evolutionary Art Are..., one major difficulty with this approach is of fatigue since the artist, or others, have to constantly judge the 'fittest' individuals amongst each successive generation. This fatigue limits the space of the research in terms of its depth and size, expressed in the number of generations explored and in terms of the size of the populations [101].

To cope with the 'fitness bottleneck' presented by this approach, some alternative strategies were undertaken, such as transferring the evaluation of the fitness to a crowd that is present in the space of the work [223]; or is remotely accessing the work through the internet [84].

The most explored strategy is, however, transferring the aesthetic evaluation to the computer $^{3}$. The first to try this process was Bajula et al. [30], who took advantage of Karl Sims' database of interactions with the audience to train a neural network, which he later used to evolve images without human intervention [105]. In these models, combining human agency with computational autonomy, a subset of the population is evaluated by human intervention

\footnotetext{
${ }^{3}$ These approaches to beauty (fitness) are informed from a Kantian perspective, where the experience of art is a product of the perceptions of the subject.

Theodor Adorno, however, criticizes this stance, suggesting an alternative perspective where it is in the shared experience of object and subject that beauty is revealed. Adorno combines the Kantian emphasis on the materialistic object with Marx's role of art in society and Hegel's emphasis on its intellectual aspect. In this view, the subject observer is not neutral, but he/she also is part of a system external, yet intersecting the one formed by artwork and the observer.

The neuro-scientist Ramachandran has reopened this discussion recently, suggesting 10 universal laws of beauty which are common to all human cultures [164]
} 
and this subset is then extrapolated to the entire population through computational agency. Takagi, Yuan and Machwe [231, 148, 258] provide overviews of works combining these two approaches.

Another popular approach to autonomous measurement of aesthetics is the analysis of the works through their phenomenological nature, the scrutiny of the syntactic elements of the visual works in the form of shape, colour and composition, isolating the structural relations from their semantic context and meaning. Overviews and discussions are provided by McCormack and Galanter [164, 101].

Independently of whether the aesthetic evaluation is hybrid or entirely computational, all the methods described have shown limited success so far. Works tend to resemble specific artists, artistic goals or styles, and are medium specific [101].

In his set of open-problems for EvoArt [162], Jon McCormack lists the measurement of human aesthetic judgment. Arguing along similar lines, Galanter proposes an alternative research situated in neuroaesthetics ${ }^{4}$, and in particular in Martindale's model of neural networks ${ }^{5}$. In this way, systems would incorporate the idea of an observer into the evaluating model.

\subsubsection{Cellular automata, the second class}

The technique described so far provides a rich methodology to create still imagery, or videos showing the structural evolution of the forms such as McCormack's Turbulence and Latham's video show.

In the late 1970s, Paul Brown developed a significantly different exploration. With resonances from op-art, Brown's prints can be described as compositions in a two-dimensional matrix where individual elements interconnect and relate with their neighbours. He based his works on a technique called Cellular Automaton, which I argue provides the second paradigm for EvoArt. CA are an extension of Finite State Automata (Turing) and Self Replicating Machines (von Neumann) ${ }^{6}$, concepts from which CA inherits.

Rather than interacting with a fitness function, individuals in Cellular Automata react to their environment. Usually disposed in one-dimensional or two-dimensional grids of cells,

\footnotetext{
${ }^{4}$ Neuroaesthetics (or Neuroesthetics) is a scientific discipline that uses the techniques of neuroscience in order to explain and understand, at the neurological level, the aesthetic experiences of contemplation and creation of works of art [259].

${ }^{5}$ Martindale cites 25 empirical studies of specific aesthetic experiences compatible with this model of neural network. However, to my knowledge, no one has yet implemented this model [153].

${ }^{6}$ The device idealized as a self-replicating machine would follow instructions to create a new copy of itself. The concept was then extended by Von Neumann's friend, Stanislaw Ulam, also a mathematician, who suggested the construction of this machine in paper as a collection of cells on a grid.
} 
individuals, or cells, have inscribed a set of rules defining their different states and forms by which they will react to the changing states of their neighbours. In discrete time steps, all the cells in the grid change their current state according to these internal rules. Global patterns on the scale of the grid emerge from the local rules of the cells.

One of the most well studied cases of emergent phenomenons in CA are Gliders. The spontaneous formation of the Glider pattern was first reported in the famous Game of Life. Gliders are a set of 5 cells that seem to act as one single unified entity. The interesting fact is not only that no one has explicitly coded this behaviour in the higher level; as in the lower level, in order to keep the glider alive, cells need to constantly switch their state (from active to inactive and vice-versa). This phenomenon of higher-level behaviours emerging from lower level rules is known as emergence.

To facilitate, I have described here the simplest form of CA, for extensive overviews of CAs, refer to Wojtowicz and Mirek [255] and of CAs and sound to Burraston and Edmonds [57]. In the arts, CA can be found in the 'bions' in Brown's Biotica [55]. Verstappen's landscapes, makes use of $3 \mathrm{~d} \mathrm{CA}$, which make use of proximity rather than adjacency. When combined together, these two first paradigms provide a framework combining simplicity and a resulting complexity where individuals can be represented using a genome-like encoded description, which is then translated into a visual representation while simultaneously perceiving their environment and reacting according to a set of predefined rules. If this set of rules is inspired by nature and implements some sort of metabolism and life cycles, creating the conditions for reproduction, we are in the presence of a very powerful framework, which forms the basis for dynamic EvoArt with co-evolution.

\subsubsection{The third class: co-evolution in multi-agent-systems}

Contrasting the discreetness and spatial rigidity of Cellular Automata, agents can move around in agent-based-models ${ }^{7}$ unconstrained, exploring their environment ${ }^{8}$. Each individual in the environment is described by an algorithm specifying a set of rules, which determines the agent's behaviour and its interaction with the environment and other individuals. Therefore, each individual in the population is represented internally by a data-structure where variables describe their current status. The environment is perceived via sensors, and a set of actua-

\footnotetext{
${ }^{7}$ These models where individuals, the agents, have their own strategies and evolve according to their interactions are called Agent Based Models (in AL's literature), also known as Individual based models (in Ecology's literature). Other systems ruled by interacting individuals that are considered to be agent based include particle systems and lattice gas models.

${ }^{8} \mathrm{CA}$ also 'move', but in prescribed ways. Gliders, the emerging structures from some CA also appear to move in the space of the matrix.
} 
tors enable some levels of agency and responsiveness providing independent decisions, which satisfy the internal goals. Agents must adapt to dynamic spaces, where the environment is also composed of other evolving agents. This autonomy from the agents in the world is what confers a unique richness to these models as agents explore their space-scape, interacting with the environment and each other.

Packard distinguishes as intrinsic adaptation this type of evolution, occurring as a result of interacting subsystems, from the extrinsic adaptation at play when the evolution is dictated by an explicit fitness function as in the case of the first paradigm (Gtype-Ptype) [183]. Intrinsic metabolic evolution is used in closed autonomous models of ecosystems [257, 97, 22], or even in more complex models where the user participates in feedback mechanisms, driving the metabolic path of the virtual population [225, 158, 19].

The above mentioned Polyworld, from Larry Yager, provides an illustration of this combination of paradigms. Agents have a) a genome describing their physiognomic features such as their dimensions; b) a metabolic system regulating their life, and consequently their need to feed to gain more energy to move; and c) a simulated visual system which feeds a neural network that is used to interpret the external world and distinguish between what is food and what is another agent or an obstacle. From a simple set of predefined behaviours, a complex set of behaviours emerges where distinct behavioural niches can be observed, such as individuals that specialize in moving exclusively on the edges of the environment or those that find it found more beneficial to move in circles [257].

This class (or paradigm) opens the door for the development of evolutionary strategies at the level of the individual. Dorin and Whitelaw's chapters on 'art using artificial ecosystems' [80] and 'cybernatures' [251], respectively, offer two comprehensive accounts on these works where agent's interactions are at the core of the practice and function as generative methodologies for novelty and surprise. Agency might be implemented using a rule-based system. However at this level, two technologies are most distinguished with regards to the individual ability to perceive its habitat: neural networks and classifier systems.

\section{Neural Networks}

Another important sphere of research in the 1990s has put a great deal of attention on the individual level of simulation: the morphology of the individuals and their behaviour. In terms of behaviour, learning became a central aspect of research. Models started to include physical forces inscribed in the environment. How could the creatures learn to cope with different physical forces: swim, walk or crawl? How could the creatures learn to move and simultaneously optimize their movements? One technique appears to be favored: neural networks. 
An artificial neural network (ANN) is a data structure inspired by the brain's neuronal connectivity and functioning. Its roots lie on Connectionism, and on the fundamental discoveries from Ramón Y Cajal, around the period of 1890s, that the brain contained an immense array of interconnected specialized nerve cells [198]. Moreover he uncovered that these cells were connected by long branching axons and dendrites and possess fascinating electrical properties. In 1965, drawing on these concepts, McCullock and Pitts suggested that neurons could be understood as simplified on/off logical gates, and this would enable their instrumentalization to compute logical operations [167].

An artificial neural network is composed of a network of processing elements highly interconnected like neurons. These 'neurons' emulate the physiological synaptic processes, operating as logical gates. Consequently, they filter the incoming input activating, or not, all the subsequent neurons to which they are connected.

For convenience, I will describe feed-forward neural networks, the simplest form of ANN. In one extreme of the network, external sensors connected to input neurons activate and initiate the operation. In the other extreme, another set of neurons is connected to actuators performing tasks and operations. For instance, in one extreme we can have sensors perceiving some hypothetical creature's balance, its position towards a light source and contact sensors. In the other extreme, we have the rotation and the degree of freedom of the member's limbs.

Depending on the type of network, multiple hidden layers might exist in between these two external layers. In addition to the input of the sensors, looping connections enable the formation of a short term memory. This memory is the key factor in detecting the recurrence of patterns. If the input data demonstrates repetitive patterns, then the network will settle into an attractor state. If the agent transits to another repetitive pattern, the network may settle into a different stable attractor state [189].

Neural networks are used extensively in AI and consequently in the arts involving AI. The aforementioned geometric individuals in Polyworld [257], the creatures from Karl Sims [222] or Starfish from Richard Brown [55] all provide examples of the extensive use of neural networks as models for individual agency.

\section{Classifier Systems}

A second important technique for an agent's learning process are classifier systems. These are adaptive systems, first developed by John Holland [119, 118], combining reinforcement learning and evolutionary computing. This model of agency provides an alternative solid methodology to equip agents with learning skills. It can be seen in diverse models of animats $[97,254]$. 
A set of sensors equips agents with the ability to perceive the external world and a second set infers the internal status (or health) of the agent. Each sensor, when active, triggers a message, which is posted on a message board. This message matches the pattern of the index of one or more rules in a rule-table and each of these generates subsequent actions.

These actions are stored in an active table waiting to be executed, and they can take the form of actions being performed or new messages being generated. Each of the rules in the rule table has a credit or priority assigned. As such, all these actions in the active table have a priority.

At each time-step, the message with the highest rank is performed, either generating more messages on the message board or activating actuators, which will perform some sort of action. Learning is achieved by means of rewarding, positively or negatively, the actions according to some measure of success on the achievement. For instance, when the agent is hungry and manages to feed, the rules which led to the activation of this action of feeding change their priority credit, adding a positive reward.

Besides the process of assessing and rewarding existing rules, classifier systems might also generate new ones. Using a procedure inspired by genetic algorithms, new rules are generated through a process of mutation and recombination of the previous rules. Genetic operators of mutation and crossover are applied to existing rules to generate the new ones. The rule table is then updated, discarding useless rules from the 'rule-pool' and adding the effective ones.

Holland [119] illustrates the operative procedure from a performance system in a classifier system with what is now a classic example, describing a frog waiting for its prey (see Algorithm 3.1):

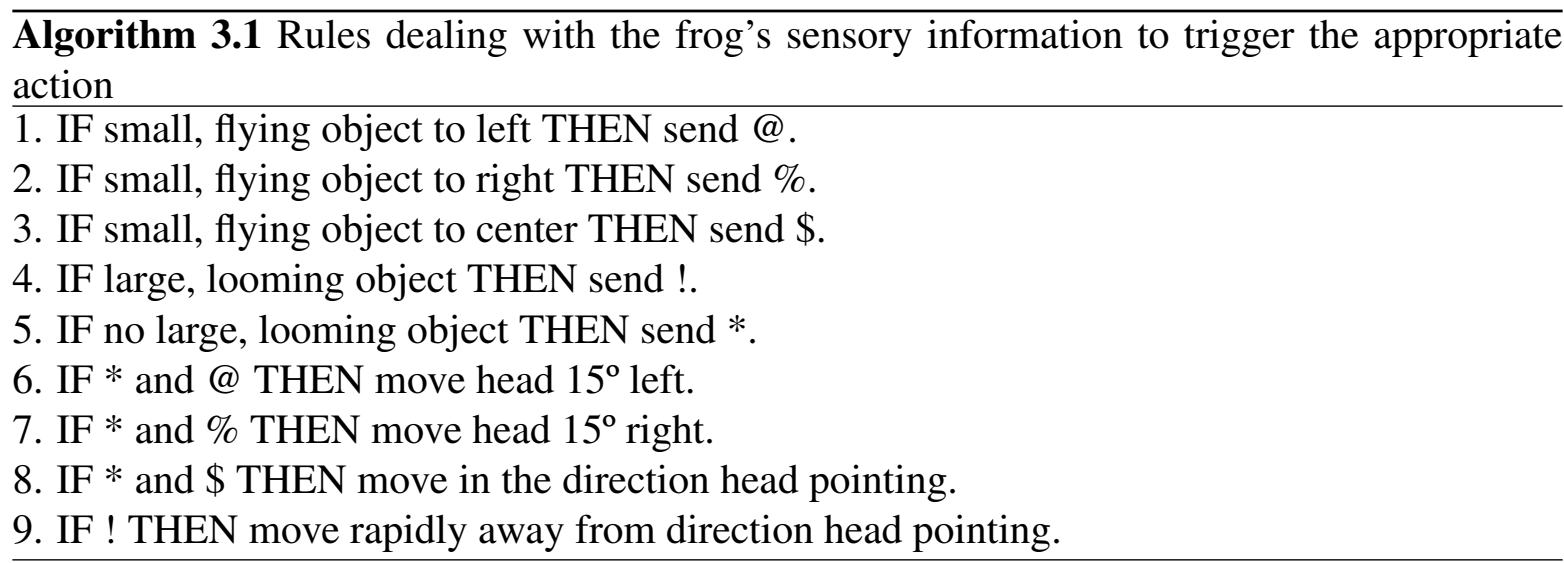

The presence of the messages * and @ on the list indicates that a small object is flying on the left and that no large looming object is around. This combination would trigger the sixth rule, making the head move to the left by $15^{\circ}$ [119]. 
McCormack's Eden and Antunes and Leymarie's Senhora da Graça [158, 22] provide examples of classifier systems at play in CEs.

\subsubsection{Other important techniques}

\section{Swarm algorithms}

In 1993 at SIGGRAPH, Ken Rinaldo presented The Flock, a group of interactive and robotic sound sculptures demonstrating behaviours analogous to flocking in natural groups, such as birds and fish [209].

The technique specified is called Swarm algorithms, and it was initially developed by Craig Reynolds. He discovered that simple individual rules of interaction could simulate the complex movements of schools, herds and flocks, suggesting that these group behaviours are emergent [206, 207]. Each of the individuals has a perception of the behaviour of a reduced group of neighbours.

A standard swarm development consists of five core rules: a) separation, move away from neighbours when these are too close; b) alignment, approximate to the average velocity of the neighbours; c) cohesion, move closer to other neighbors; d) curiosity, approximate to objects of interest and e) society, communicate with other individuals in the social group.

Tim Blackwell distinguishes two types of swarms: non-social and social [43]. In the first of these, the individual dynamics in the group depends only on the individual spatial configuration. In social swarms, on the contrary, individuals have the ability to communicate with others independently of the distance at which they are from each other. Social swarms are usually employed as problem solvers. A good survey is provided by Al-Rifaie [12].

Swarm particles can be extensively found in visual effects in the movie and $3 \mathrm{~d}$ industries (movies, such as Batman Returns or Lion King, and video-clips from Sigur Rós, or in atmospheric effects, such as raindrops, often seen in advertisements).

Diverse interactive 3d-world artworks, such as $x T N Z$ [19], EIDEA (Environment for the Interactive Design of Emergent Art) from John Mitchell and Robb Lovell [173] and The Bush Soul by Rebecca Allen et al. [169] also make use of flocking objects. Other forms of artistic use of swarm intelligence can be found in Blackwell's granular compositions, in the robotic painters from Leonel Moura, or in Paulo Urbano's drawings using the cooperative behavior using stimergic mechanisms as seen in ant colonies, [176, 242, 43, 243, 10]. In Art Made From Artificial Ants, Nicolas Monmarché et al. describe a selection of projects that utilize the spatio-temporal structures created by swarm intelligence and self-organization in sonic and graphic settings [174]. In another chapter of the same book, Christian Jacob describes 
his work with team mates Jeffrey Boyd et al., articulating swarms with interactive artificies, such as the audience's gestures of devices (a fan, electronic toys), that allow the influence of external factors in the swarm behavior by changing structuring parameters in the swarm. More interesting is their use of an evolutionary approach to breed or design 'choregraphed behaviours' which combine swarming agents with developmental programs that extend Lindenmeyer systems (discussed in the next section). This results in artistic snapshots of the arrangements of the agents in the swarm [127].

\section{Grammar based techniques: L-Systems.}

In the research on the generation of morphology, grammar-based-models are distinguished as other powerful and commonly used standard techniques. The morphologies of the entities are described in alphabets of symbols. Each of these symbols is associated with a visual object or transformation.

Lindenmeyer's L-Systems is probably the most known grammar based methodology. LSystems were initially developed by Aristid Lindenmeyer, a botanist and also assiduous frequenter of early ALife conferences, as an abstract mathematical model for simulating the development of multicellular organisms [195]. One initial axiom contains the initial state or seed. A set of parallel if-then rules, called production rules, describes how symbols in the axiom are rewritten with other symbols. At each discrete time step, all the symbols used in composing the axiom are replaced with new symbols described in the rewrite rules.

For instance:

$\overline{\text { Algorithm 3.2 a) The initial axiom b) Rules describing a replacement of symbols c) The } 3}$ first replacements

1. a) Axiom

2. $\mathrm{Fr}$

3. b) Productions:

4. $\mathrm{F} 1->\mathrm{Fr}+\mathrm{F} 1+\mathrm{Fr}$

5. Fr $->\mathrm{F} 1-\mathrm{Fr}$

6. c) 1st rewrite: F1-Fr

7. 2nd rewrite: $\mathrm{Fr}+\mathrm{F} 1+\mathrm{Fr}-\mathrm{Fr}$

8. 3rd rewrite: $\mathrm{F} 1-\mathrm{Fr}+\mathrm{F} 1+\mathrm{F} 1-\mathrm{Fr}-\mathrm{F} 1-\mathrm{Fr}$

The robustness of the model is not limited to drawing botanic-like forms by means of logic. L-systems themselves do not generate images, rather long strings are generated. These need to be interpreted. Due to this interpretative flexibility, L-Systems have been used not only to create artificial plants, flowers and trees [145, 195, 161], or virtual creatures [121], but also in 
areas not so obvious, such as the production of designs for furniture [122], structures enclosing space in architectural design [54], procedural design of cities [184], or even as techniques for data compression [180].

Bruce Damer et al.'s Nervegarden [69] and Jon McCormack's renowned installation Turbulence $[156,160]$ make extensive use of this technique in the creation of fantastic botanic forms, which populate their works.

\subsection{Interactive aquaria: A participative audience}

As mentioned earlier, the first developments of virtual environments considered the space as an 'out-there', where the action unfolds. This granted the audience the role of mere passive observers, excluded from the simulation. With time, the space became hybrid and opened to a more participative audience. In this realm, the roles of experimental artists and the games industry were pivotal for the development and popularization of ALife.

A second strategy of design involved letting the participants interact with the virtual environments. Software platforms used to study the evolutionary biology of self-replicating entities such as $[9,93,172]$ allowed, to some extent, the configuration of the macro-features of simulation. Direct interaction with the creatures was introduced later in works such as Jeffrey Ventrella's Darwin Pond and Gene Pool [244], and in these environments the audience could drag a creature, placing it next to food or next to a mate, and so on.

Interfering with the course of the creatures evolution, changing their parameters and their life conditions during the entities' lifetimes, or even designing and populating the world with entirely new plants [69] or creatures [225, 170, 193] has more recently, become a common practice in nurturing games and installations.

Games traditionally explore the emotional engagement with virtual creatures in participative products. In these products, the audience is usually responsible for nurturing and coevolving virtual creatures. In the mid 1990s, nurturing and breeding synthetic forms of life became mainstream. The iconic Tamagotchi is one of the most representative developments of this period [31]. This keychain creature required permanent attention since it needed to be looked-after for 24 hours a day. The transportable pixel creature reached a status of wideworld sensation around 1996.

However, the most complex example of this subset of games was perhaps Steve Grand's game Creatures. In this popular game, the audience could hatch virtual eggs, growing and nurturing alien-like creatures. Each creature, a Norn, had individual features and behaviours distinct from any other; these traits were inherited from previous generations and evolved in a 
process of genetic mutation. Each Norn had a virtual brain in the form of a neural network and a digestive system. Users had to educate them to feed and to communicate (via the exchange of a few words). The habitat was enclosed in standalone computers, however the audience could share eggs with other virtual locations through the internet [104]. These primeval examples of virtual pets illustrate a vigor that soon spread, giving rise to the myriad of virtual pets (as diverse as dogs, chickens or aliens) that we can find nowadays online and offline [92, 5].

The most recent success in this area is Will Wright's game, Spore [26]. In Spore, the player controls the development of a species from its beginnings as a microscopic organism, and throughout its different stages of development as an intelligent and social creature, ending in an interstellar exploration as a space travelling culture ${ }^{9}$. Spore has drawn wide attention for its massive scope, its combination of genres (including action, real-time strategy and RPG), its use of non-linear game-play ${ }^{10}$ and procedural generation. In each of the stages, players are able to use various content-producers to create new assets for the game. These can then be uploaded to Sporepedia, an online repository, and later downloaded by other players [27].

\section{A cybrid ${ }^{11}$ nature}

In 1996, TEO was created by Fujitsu. TEO (The Other Earth) was a overelaborate environment, a habitat for a strange half-dolphin/half-bird creature called Fin-Fin, which interacted with participants and was capable of remembering previous experiences [99]. Fin-Fin interacted by singing and doing acrobatics. Before that, users had to patiently conquer its confi-

\footnotetext{
${ }^{9}$ The evolutionary approach used in Spore is problematic to a diverse extent. In the tribal stage, for instance, players can collect points when performing tricks or fighting with other tribes. These 'DNA-points' can then be converted into new body features that the player can effectively buy or sell and apply to the tribe.

${ }^{10}$ I was cautious here to avoid using the term 'open-ended game-play', commonly used in the games industry, which might introduce some confusion with the concept of 'open-ended evolution' in the domain of evolutionary computation. Open ended gameplay refers to prescript challenges that can be completed in a number of different sequences.

${ }^{11}$ Patrick Lichty refers to Cybrids and Evergents in works which live in a hybrid domain that merges the virtual and the tangible [147]. The term Cybrid identifies works which live in both realms simultaneously. This is distinguished from Evergent in the way that it applies to works originated in the virtual that materialize in the tangible, respectively. An example of the Cybrid is provided by Micha Cárdenas (aka Azdel Slade in Second Life). Cárdenas is a transgender performance and new media artist, whose work deals with bio-politics. In her work Becoming Dragon from 2007, she deals with the interplay of biotechnology and virtual reality to juxtapose the idea of embodiment by proxy in the avatar with the mismatch of body and identity in the transgender subject. The work was an ironic comment to California's law on transgenderism. This requires transgenders to live 365 days in a transient state before getting a gender confirmation. In her work, she lived for 365 hours immersed in Second Life embodying a dragon. In a loophole, a motion capture system translated her movements at a computer lab at San Diego University, into the virtual space of Second Life, which she perceived via a head mounted display [60]. In contrast, examples of evergent works are provided by the sculptures produced by Driessens and Verstappen from digital processes running in the computer and originating tangible forms [246] that are exhibited in art galleries.
} 
dence by waving, speaking, singing or even playing musical instruments using an interface combining a microphone and camera.

This type of interaction resonates with installations from experimental artists from the same period, where the virtual and the physical appear combined. In Christa Sommerer and Laurence Mignonneau's Life Spacies series of works from the 1990s, systems were composed by the entities in the virtual environment and an audience was present physically, in the museum, or virtually, on the internet. Participants interacted with the creatures, promoting actions such as cloning and mating. Text from e-mails sent by the participants was converted into genetic information and inducing the formation of newly born creatures into the world [170, 228]. Similar technologies of motion capture were explored in Life Spacies, and these enabled a calculation of the physical presence of the audience in the space, whose presence affected the behaviour, development and evolution of the virtual entities in the world.

This extension of the virtual environment to the internet is also at play on Jane Prophet's Technosphere. As a form of enhancement of emotional engagement, virtual creatures inform their creators (via e-mail) of their latest developments in the world [193].

Generally, audiences have a positive feedback on these works. In Biotica, reproduction only happens in the presence of users [55]. In Eden, the physical movements of the bodies in the space surrounding the work influence the growth of virtual food [158]. In Meniscus, the creatures grow more or less stressed according to the audience's physical presence, which affects the level of water on the simulation [76, 80]. Also, in A-Volve the audience can also interfere with the predator-prey interactions as the virtual creatures run away from hands touching the screen [226].

Latest developments in interactive technologies include the Wii and the iPhone. A vast array of Virtual Life games, such as Worlds of Zoo or Sim Animals, can already be found exploring these new features ${ }^{12}$ [92]. The recent success of Farmville has shown that other, not so obvious routes, such as the social-community Facebook, might be useful platforms to promote virtual communities of living agents ${ }^{13}$.

The inclusion of different forms of interaction with virtual worlds have allowed a transformation of status of the audience from passive observers to active participants. The works become inclusive open systems, where the delimitation of territories from physicality and virtuality is getting more and more blurred.

\footnotetext{
${ }^{12}$ Earlier instances of mobile pets, such as Bandai's Tamapichi and the WAP version of Tamagotchi, Wapagotchi, explored the technology of the mobile phone. The first allowed users to transfer their creatures to be cared for and nurtered by their friends, and the second allowed the creatures to send sms messages to their owners requesting food.

${ }^{13}$ I am using the example of Farmville only relative to the biological metaphor used. Farmville is by no extent an evolutionary game in the computational science sense of the term evolutionary.
} 


\subsection{Final notes}

Peter Bentley and David Corne [36], first, Penousal Machado and Juan Romero [210], later, and more recently Stefan Bornhofen, Jean Heudin and Alain Lioret [49] have edited collections of foundational texts overviewing EvoArt from a perspective centered on a technical point of view. These works are complemented by Whitelaw's Metacreations [251], a critical development providing an in-depth portrait of the practice of ALife art.

This thesis further refines this epistemology focusing on CEs. Throughout this chapter, I have analyzed the materiality of the processes of EvoArt, unfolding a technological teleology in order to acknowledge the standards used in the construction of these artefacts from a technical perspective. Three paradigms informing EvoArt were enunciated, assisting in understanding how developments in AI have driven the formation of the fundamental techniques for EvoArt. CEs emerge from this framework as complex evolutions of the original genetic algorithms, where the fitness of the dynamic agents is exogenous ${ }^{14}$, defined by how well these agents/individuals perform on their habitat.

In the following chapter, I will discuss these artefacts as cultural forms.

${ }^{14}$ HEre I use exogenous as opposed to the endogenous fitness from GA, where this evaluation criteria is explicitly stated. 


\section{Computational Ecosystems as Cultural Forms}

'I am an aspiring, latter-day Baron Frankenstein. Like him, I believe that life can be created where there was none before. Like him, I think that it is possible to make thinking, caring, feeling beings that, when these beings exist, it may be reasonable to ascribe them a soul. Like him, this is what I have set out to do.'

Steve Grand, in Creation: Life and How to Make It

\subsection{Introduction}

In the previous chapter, the set of techniques forming the fundamentals of the palette used in the construction of artistic CEs were identified. The present chapter complements that research by examining the cultural framework that informs this practice. In particular, it emphasizes how ideas of self-organization, feedback, autopoiesis and emergence, associated with the aesthetic affiliations of EvoArt with Generative art, come to inform the bottom-up methodology of construction of the works. In other words, this chapter looks at the 'technical support' (using Krauss' terminology), or the set of ideas forming the framework of this practice.

\subsection{Aesthetic affiliations of Evolutionary Art}

Amongst the great cultural and social changes that occurred throughout the 20th century, in particular during the period of the post-war, Cybernetics [252] and Systems theory [40] have shifted from an essentialist paradigm towards the recognition that not only objects require study but also the relationships between the objects. In the arts, this transition would have notorious influence in artistic movements such as Kinetic art, Minimalism, Processual art and Op-art. In Beyond Modern Sculpture, Jack Burnham argues the 'disappearance' of the objet d'art and the consequent growing attention devoted to the system in the visual arts tradition from the Euro-American cultural axis [56]. Burnham's 'system aesthetics' draws the shift in 
attention from self-contained objects characteristic of modern formalism, to a post-modern open-ended and immersive experimentation. From a historical perspective, Generative Art (GenArt) and ALife-Art seem to inherit and operate within this lineage. Rooted in an algorithmic and systemic understanding of the artistic artefacts, these practices take expression in rich generative systems which may evolve dynamically as result of their internal dynamics.

\subsubsection{Open systems}

\section{Generative art}

In Japanese Renku, a form of linked poetry, or even Desgarradas, a form of folk singing from Portugal, two or more performers answer and challenge each other, following a shared system of rules. In Desgarradas for instance, each stanza needs to answer the previous, and within the interval of one or two tones, and within a rhyme, a new challenge is improvised to trigger the following singer.

These are artistic expressions that may be described as Generative art, a category commonly associated with art forms characterized by the adoption of 'the system' and 'the process' as main foundations; artworks making use of autonomous systems in the production of the artefacts, be it visual, audio or textual forms.

Traditionally, Generative art (GenArt) is characterized by its algorithmic nature and, more precisely, by the existence of a set of constructive rules which drive and unfold the workgenerating novel forms and surprising developments ${ }^{1}$.

A number of common patterns and techniques are identified and characterize this practice. The idea of variation over one single instance (physical object, musical sequence, syntactic structure) is one of these. The way this exploration happens is by i) variation over one or more parameters or ii) permutations and recombinations in the structure. Another recurrent idea is the use of operators of symmetry, or changes in scale and order. These strategies allow explorations in the domain of possibilities contained in each of the individual variables, the parametric landscape ${ }^{2}$.

A reading of Galanter introduces a formalization of Generative Art which is inclusive and almost universal to the whole of art history. In his eyes, generative works are those in which

\footnotetext{
${ }^{1}$ GenArt, from this perspective, is as old as art, as Philip Galanter advocates. Evidence of autonomous systems driving the construction of artefacts is provided by practices as old the instruments found in caves and dated as far back as 70000 years ago [100].

${ }^{2}$ Parametric landscape is understood as a virtual space in which a certain object, say constituted by 3 variables - $\mathrm{a}, \mathrm{b}$ and $\mathrm{c}$ - occupies a single dot of that three-dimensional space. Varying the value of one of its parameters gives us a second point in that space. The variation of all the values in all the parameters allows the total exploration of the mentioned space.
} 
origin resides in a systematic execution of a set of explicit rules. Works laying their bases according to these premises are diverse and appear in multiple disciplines, from the visual arts to music and literature. Examples include: the geometrical abstractions from traditional Middle Eastern visual arts and architecture; many of the works developed during minimalism in contemporary visual arts; serialism in music; or even linked poetry. All of these disciplines share an emphasis on the processes used during the construction of the work. These processes take the form of rules (the algorithm) and works are generated when the system (the interpreter/performer, in the form of a person or machine) executes these rules systematically.

Musical composition has indeed a long tradition in the use of generative methods, with major expression in the Gregorian chant or the art of fugue from Bach. Authors from the second Viennese school, the serialists from the post-war era, or yet Cage, Xenakis, Stockhousen or Eno have established through their practice a vast and fertile exploratory field, and their work can be used here to illustrate the amplitude and importance of generative systems to musical composition. In the Opus 23 series, for instance, Shoenberg the promoter of dodecaphony, defines 12 notes by the existing intervals between each. The same sequence is played repeatedly forward, backwards, upside-down ${ }^{3}$, and backwards and upside-down, and these are articulated with changes in rhythm, dynamics, timbre, orchestration, and so on [120].

In contemporary visual arts, generative processes can also be identified at play, driving the materialization of a significant number of works. In particular with the Minimalist movement, generative processes have been adopted and are at the genesis of pieces from authors such as Josef Albers, Frank Stella, Dan Flavin or Sol Lewitt. In Homage to a square, for instance, Josef Albers has explored variations in tone and size upon the same subject, a set of solid rectangles [130]. Using a similar methodology, Frank Stella's Black Painting and the Protractor series can also exemplify the vast outcomes of the generative procedures in this artistic realm $[66,65]$.

Dynamism With the advent of electronic media, and in particular the computer, a new generation of generative works articulate the classic generative approaches in explorations on the realm of time, works which take the form of autonomous, dynamic and interactive complex systems. These integrate the tenets of Minimalism, emphasizing the gestalt of elements actively participating and forming a holistic composition, with principles of dynamism earlier enunciated by artists like Alexander Calder or Jean Tinguely [29, 13]. In Calder, the time, the wind or the rain challenge the stasis of the modern sculptural object. Calder's mobiles

\footnotetext{
${ }^{3}$ In music, 'upside-down' is defined in terms of intervals, the same sequence is played with the distance between two adjacent notes being inverted, for instance if this distance is of (+) two semitones it then becomes (-) two semitones.
} 
are sculptures made of articulated modules, and dynamics become a central element for his design. These works interact with the natural elements and generate variations in time. The effect of wind changes the sculpture's spatial arrangement, only constrained by the levels of freedom of the joints connecting the separate modules. Later, in the tradition of Happenings from the 1960s, Tinguely explored what can be described as performative objects, a combination of sculpture and event. Part of his work are happenings with 'metamechanics'. These are self-destructive machines, kinetic sculptures with a narrative and a limited lifetime.

If these works abolish the classic idea of the pedestal and challenge the modern precepts of the art object, with some of the more recent digital dynamic generative artworks, from Lia [4] or Golan Levin [141] for instance, the stasis of the object becomes definitely compromised and systems integrate in their conceptual framework, the fourth dimension of time, with permanent change and evolution.

Indeterminacy The modus operandi of these artists seems to rely much on the uncertainty that these dynamic works provide. This imprecise and almost organic level of indeterminacy is closely aligned with ideas enunciated one century ago by Marcel Duchamp. One of the great foundations of contemporary art is his work, The Great Glass (Grand Verre - 1913-25). This is a monument to chance, exemplary for the sense that Duchamp gives to the role of random events during the process of art making. The delicate colours of the sieves, for instance, were the result of varnish applied to accumulated dust. For the draft pistons at the top, Duchamp used a square net that he got distorted by the flow of hot air when he hung the net above a radiator. This distortion was captured photographically and the shots were distributed with a pattern produced with a toy cannon launching paint-dipped matches. Previous to the Great Glass, Duchamp did the work 3 stoppages. This was presented as wooden templates resulting from the shape and dimensions of threads fallen according to Duchamp's instructions: 'if a straight horizontal thread one meter long falls from a height of one meter onto a horizontal plane distorting itself as it pleases and creates a new shape of the measure of length.... The shapes of the threads were used to produce the wooden templates, and these were used in the Great Glass to produce lines connecting the bachelours to the sieves. This emphasis on the character of chance was reinstated when Duchamp, years later, incorporated into the work a transport incident that broke one of the glasses [126].

As Margaret Iverson points out, the performative aspect of Duchamp's ideas were recovered by music composer and theorist John Cage during the 1960s [126]. Cage's interest in indeterminacy is illustrated by his use of I Ching coins to generate musical compositions, or in his later works when he leaves great freedom for the interpreter. Rather than a melodic linear mu- 
sic experience, Cage is much interested in chance events occurring during the performance and in creating fields of resonating sounds, emphasizing what we see today characterizing granular composition made with swarms for instance. Cage had a great influence on the cultural scene during his time and came to mentor artists such as Allan Kaprow and Robert Raushemberg, who would establish solid art movements much interested in breaking with the 'art bourgeois' and reduce the distance between art and life. The interest of these movements from the 1970s, in particular Fluxus, in indeterminacy came to inform many contemporary practices. Amongst these is Evolutionary Art, an art form that also looks at other formal and processual aspects of life.

\section{Evolutionary art}

In 1859, Charles Darwin and Alfred Russel Wallace shocked the world with their explanation of the origin of species, arguably the most categorical scientific paradigm change, in Kuhn's terms, in the human history. Around one and a half centuries later, a significant number of artists fructify this revolutionary idea, combining it with further developments from scientific knowledge to produce artistic works. In particular, neo-Darwinist accounts, inspired by Mendel's genetics associated with the notion of emergence ${ }^{4}$, provide the means to formalize a set of techniques driving artistic production. Indeterminacy is again central in processes of scrambling and mutation of genes that are at the core of the practice to guarantee complexity and diversity.

This sub-genre of GenArt, which is inspired primarily by evolutionary computing, has appropriated principles of natural selection and evolution to drive the evolution of artworks in computers epitomized by Margaret Boden and Ernest Edmonds as Evo-Art [44] (here called EvoArt). This practice takes expression in an eclectic set of formats, including still digital imagery, or evolving $2 \mathrm{~d}$ or $3 \mathrm{~d}$ environments, which are experienced by an audience either in intimate experiences in personal computers or in large scale immersive installations.

If Holtzman's work emphasizes the structuralist foundations of historical GenArt [120], in turn, Manovich asserts that as a result from a scientific paradigm shift, the contemporary expression of this art form is distinctly concerned with complexity ${ }^{5}$ [150]. Mitchell Whitelaw

\footnotetext{
${ }^{4}$ Emergence describes the phenomenon when, in a system, the whole is more than the sum of its parts; when macro-patterns occur at higher levels of the system caused by the interactions of micro-patterns at its lower levels. These macro-patterns occur without any explicit inscribed description of the phenomenon at these lower levels [175].

${ }^{5}$ In Language of New Media, Manovich argues the appropriateness of thinking generative abstract art as symbolic representations of the new image of the world 'as a dynamic network of relations oscillating between order and disorder - always vulnerable, ready to change with a single click of the user'. Unlike the paradigm of reduction that characterized art from the first half of the twentieth century, he contends that 'visual arts
} 
confirms this shift towards complexity and connectivist accounts, asserting these as the referentials for ALife art's framework where self-organization, autopoiesis ${ }^{6}$ and emergence take an eminent role in the artistic discourses and practices [251]. ALife-art appears, as such, to culminate Burnham's account on art history: works are substantiated in systems that are complex, bio-mimicking and autonomous.

A number of authors have surveyed this practice $[210,251,44,36]$. Amongst them, Whitelaw, in Metacreations [251], analyzes the artistic genre of ALife art. He suggests that ALife art appears as one of the latest forms of a long tradition of artistic attempts at capturing nature, either in the external appearance or in the internal processes. According to his view, the instrumentalization of biological processes, so characteristic of ALife, appears rooted in the organic ideologies from modern artists, such as Paul Klee and Kasimir Malevitch [250]. These share with this new generation of authors the dream of capturing natural processes.

What becomes critical and distinguishes ALife (and consequently EvoArt) from Burnham's systems esthetics, and generative art in general, is the confirmation of Burnham's prediction of the autonomy of the work to ways where 'the Greek obsession with "living" sculpture will take on an undreamed reality' [56]. Relatively simple systems, which characterized traditional GenArt, appear now contrasted with biological processes of life and evolution in the complex systems of ALife.

\section{Artistic Computational Ecosystems}

Amongst these new forms are virtual ecologies, the CEs. Communities of virtual individuals reside in the memory of computers. Following the animat model (introduced in Chapt. 2),

pursue new types of representation adequate to the needs of a global information society, characterized by ... new levels of complexity' [150].

${ }^{6}$ Francisco Varela and Humberto Maturana described autopoiesis as a property of life and systems. Central to the concept is that organisms function in a circular network, which makes one into another. It is noteworthy to quote them in full:

'An autopoietic machine is a machine organized (defined as a unity) as a network of processes of production (transformation and destruction) of components which: (i) through their interactions and transformations continuously regenerate and realize the network of processes (relations) that produced them; and (ii) constitute it (the machine) as a concrete unity in space in which they (the components) exist by specifying the topological domain of its realization as such a network' [154].

A classic example of an autopoietic system is provided by biological cells. A self-organizing network of biochemical components compose the cell. These in turn produce molecules, which create a membrane, a unity, the self, a boundary separating an outside from an inside. The structures inside keep operating based on an external flow of molecules and energy, maintaining the organized bounded structure that gives rise to these components.

A factory (an allopoetic system) is the contrast to an autopoietic system. It operates with external raw materials and produces other structures rather than itself (the products). 
these individuals have some level of agency and are able to change their own status and their environment. An eventual virtual creature in a virtual world can change from a infant to a mature state, it may procreate, and eventually die. All these states may also be accompanied with changes in the creature's shape and behaviour. The environment reshapes itself continuously via the dynamic and autonomous interactions of the individuals.

With virtual ecologies, the foundation for the mechanism driving this evolution lie in the internal dynamics of the complex adaptive system formed by the populations which inhabit the worlds. The structure of these systems follows a bottom-up logic common to ALife aesthetics where the complexity observed at higher level structures emerges by recursive interactions from the self-organization of lower level building blocks and rules.

Emergence is a process by which (complex) systems and patterns arise out of a multiplicity of relatively simple or not fully specified/understood interactions. Often patterns and events at a higher scale of the system occur as a result of the spontaneous and autonomous interactions at the lower scales of detail. An example is provided by snowflakes forming their unique complex symmetrical patterns as a result of their passage through the differing temperatures and levels of humidity in the atmosphere.

In an agenda of pursuit for novelty and surprise, self-organization and emergence appears to be omnipresent in the artistic discourses [159, 55, 192]. Authors of CEs start with the building blocks and simple rules and complicate them through non-linear processes so that a complex world appears on its own. This interest in complexity and non-linearity of processes is manifest, and appears vocalized by diverse authors: 'AL has important contributions to make in our understanding of genuine novelty, often referred to under the generalized term emergence' in [159]; or 'Biotica didn't meet its original ambitious aim of capturing emergence for use as an artistic phenomenon' in [55]; or yet 'unpredictable behaviour would emerge from these rules' or 'the notion of self-organizing artificial life systems' in [192].

\subsubsection{An aesthetics of self-organization and feedback}

To address an aesthetics of emergence, it might be pertinent to recall Umberto Eco's concept of the 'open-work'. Eco, which is also strongly influenced by the new paradigms from postwar science (such as cybernetics and systems theory), suggests that works in modern music, literature and art are said to operate in a state of potentiality, of unexplored possibilities which the work may admit. An open work is not limited to a single linear reading. Its open-ended nature is complex and offers an unlimited range of possible readings; works are 'open' to continuous generation of internal relations which the addressee must uncover and select in his act of perceiving the totality of incoming stimuli [89]. For Eco, the reception of the work of 
art is both an interpretation and a performance.

Particularly critical for this discussion is the notion of 'work-in-movement', a term Eco uses to describe pieces which operate as invitations 'to make the work together with the author', works that 'characteristically consist of unplanned or physically incomplete structural units' [89]. In CEs, the building blocks of the system interact autonomously. The self-organization of processes create new trajectories. The work admits a large number of possibilities. However, each run will only see one sequence of these. The complexity of the (eco)system generates such a wide range of combinatorial possibilities that two different runs of the system will systematically diverge in outcomes. Amongst Eco's illustrations of the work in movement are Calder's generative mobiles. As in CEs, the modular structures of the mobiles preexist the viewing experience. And in a somehow similar way, the work articulates time in its substance. The Open work was first published in Italian in 1962. Five decades later, laying its foundations on emergent behaviours, and bringing the audience to intervene in the evolutionary course of the simulations [159, 19], Eco's work maintains its actuality and CEs seem to be mature instances of systemic-open-works.

Yet, despite sharing aesthetic affiliations with the pioneering artistic movements from the 1970s, experimenting in bringing 'movement' and 'change' to painting and sculpture, the ontology of CEs does not exhaust itself with the production of novelty and the exhibition of multiple scales of visual and audio complexity. CEs are more than accelerated versions of Calder's mobiles of the digital ages. Whitelaw sees ALife-art as a natural follow up of the modern attempt to imitate not only the appearance of nature but also its systems of functioning [251]. The agenda of practitioners appears to be ambitious and is inscribed with an interest in questioning life itself. The project of ALife is the chimera of exploring 'Life-as-it-could-be' [137]. Artistic uses of ALife techniques inherit this dialectic/ethos. In the words of Richard Brown, with Biotica this author wanted: '...to question... what it means to be alive' [55], or Ji and Wakefield in Artificial Nature: 'the most radical ways of Life as it could be' [248].

\subsection{The meaning of life in the systems}

This perspective of the artefacts as living systems forces a change of perspective when looking at these works, moving away from the formal and functional spheres in favour of an analysis of its ideological implications.

The strong claim of ALife is that life is not restricted to nature and ALife models can extend biology, in a similar process to what happened to chemistry when synthetic chemistry made possible the invention of new molecules and chemical compounds not previously known in 
nature.

\subsubsection{Life forms}

When Steen Rasmussen wrote the paper on his work, Venus [199] in 1990, he didn't name the individuals of his virtual community as creatures, but simply as programs. So, how did it happen that notions of life and space become such integral structures of the framework of CEs?

'When the value of variable $a$ of the agent $k l$ is lesser than a certain threshold, the list $b$ needs to be inspected to find any arbitrary agent $k 2$ whose data structure has a compatible pattern to the one of this agent. Once this other agent $k 2$ is found, then the variable $c$ of agent $k l$ must adjust its current value to that of the variable $c$ of agent $k 2$ by an $x$ amount'.

The above is the literal English translation of a set of mid level instructions from an hypothetical software program. The question is: How can this set of mid-level instructions be described in the higher semantic level as 'a creature finding a prey to hunt because it is hungry'?

The early days In 1990, when the results on Venus and Tierra were first published, a new field of Artificial Intelligence was emerging called Artificial Life. The agenda of research was focused on better understanding the limits of life by means of the study and creation of organisms and systems, built by humans, and exhibiting life-like properties. The pioneers had their first workshop in Santa Fe a few years earlier, in 1987. In the inception of the discipline, Christopher Langton proclaimed ALife as a research on 'life-as-it-could-be', a motto meant to contrast with 'life-as-we-know-it' [137]. As science once had uncovered the laws of electricity and chemistry, ALife was advocated as the research on the mechanisms of Life.

In the introduction of the Proceedings of Artificial Life II, we can read how Langton distinguishes the two main philosophical branches of the discipline: the weak claim which advocates these computational processes as simulations of life, useful for the study of aspects of life but not considered as instances of life; and the strong claim which posits that any inclusive definition of life aiming to accommodate all known forms of life will have to include classes of computational processes, which will necessarily have to be considered alive [138].

Works on the weak claim prevail in the community [233]. These works, including CEs, artificial chemistries, models of autopoiesis, self-organizing systems, evolutionary robotics and evolutionary hardware try to reproduce or get inspired in processes as they occur in nature. 
The goal of the weak claim is to understand more clearly the organic life that exists on earth, or: 'life-as-we-know-it'.

The strong claim The project of the strong-claim is more ambitious. Nature provides diverse examples of living and inert matter, which challenge our intuitive notions of what life is about. Some forms appear to be organic when they aren't at all. Others challenge our notion of organic decay and death ${ }^{7}$. There is no completely inclusive, concise definition of life itself [151]. If we ask a group of scientists for a definition of life, the answers will be biased by their original disciplines, a point James Lovelock emphasizes in Gaia: Medicine for an ailing planet. Lovelock, himself a geophysicist, describes an organism as a bounded system in a flux of matter and energy able to keep its internal equilibrium constant in composition and its physical state intact in a changing environment. A neo-Darwinist biologist will describe a living organism as one able to reproduce and adapt to its environment through a process of natural selection ${ }^{8}$. A biochemist will draw his description on the ability of the organism to take free energy, such as food or sunlight, and use that to grow according to a plan inscribed in its genes. On the other hand, a physicist will focus on the capacity of self-organization and describe the organism as a peculiar state of matter in a flux of free energy 9 .

Despite their specific points of view, all of these definitions have in common a description of life based on a number of behavioural patterns. These include responsiveness, growth, metabolism, energy transformation, and reproduction. The problem seems to be that these core patterns involve transformation or the capacity to do such. What the pioneers of the strong claim of ALife suggest is that these behavioural patterns, or processes, could also be exhibited

\footnotetext{
${ }^{7}$ In his TEO's presentation on how to recognize life in Mars, Chris Adami illustrates this formal variety observed in terrestrial nature with the growing processes seen in Crystals. Some of these, like pseudobrookite, sometimes develop in apparent filaments whose shape makes them look like living organic entities. On the other hand, a species of medusae, called Turritopsis nutricula, challenges our notion of organic decay and death. Individuals of this species metamorphose throughout their lives. In a process called trans-differentiation, the adult medusa reverts completely to a sexually immature stage when it transforms into the polyps of a new polyp colony. These polyps can then reproduce asexually, commonly using one of two ways: each one can split in two, creating two clones of the original, or it can form a colony, where the new polyps do not split from the original but rather seem to grow off its sides (budding). Various polyps in the colony may take on specialized roles. For example, one polyp may only be used for defense, while another is used for reproduction, and yet another for capturing food. In contrast to polyps, adult medusae reproduce sexually, creating a small, ciliated larva known as planula. The planula eventually settles on the sea floor and changes into a polyp, and the process starts again.

${ }^{8}$ John Maynard Smith in The Problems of Biology asserts: "Life should be defined by the possession of those properties which are needed to ensure evolution by natural selection".

${ }^{9} \mathrm{An}$ organism can be said to be alive if it a) has defined boudaries, external limits b)takes in free energy and converts it into useful work c) excretes waste products, high in entropy d) maintains a high level of internal unstability d) maintains a constant internal level, regardless of changing external conditions (Chris Adami in TED talks).
} 
by non-organic matter in the form of computational systems a fact, which they claim, positions problematically ALife models as instances of life. Through the simulation of life forms that we are not familiar with, ALife attempts to isolate the properties of life and explore patterns of life that haven't been found yet.

In inaugural models, like Venus or Tierra, individuals are abstract complexes of information, whose bodies are composed of inorganic sets of instructions. These Tom Ray calls instances, because he claims these to be instances of life [201]. Ray argues 'I would consider a system to be living if it is self-replicating, and capable of open-ended evolution' [201].

The looking-glass world The philosophical framework of Tom Ray and the other ALife pioneers is deeply rooted in a materialistic and discrete understanding of reality. Materialism endows Rationalism in that everything belongs to the physical domain and is subject to the fundamental laws of physics. In terms of life, Materialism contrasts with Vitalism, which dissociates the body and soul and has an understanding of life as a transcendental entity not subject to the laws of physics. This would be bestowed by some supernatural force to inert matter. In a materialistic conception, on the contrary, life is the result of events generated by organized matter existing in a complex and delicate balance. Life is not material, rather it is ephemeral but results from the organization of materials. Processes are organized bottom-up, including self-organized mechanisms of recursion and feedback. Organic life is organized in at least four structural levels: the molecular, the cellular, the organism, the population ecosystem ${ }^{10}$; and, arguably, additionally the geophysical planetary level of Gaia. The world is composed of a hierarchy of 'persistent structures', as Steve Grand puts it, in which life, mind, and society are all aspects of the same processual nature [103]. Individual components at lower levels of the organization interact with each other, generating differentiated structures that emerge at the higher levels. Every living being is an auto-catalytic network formed of interdependent feedback systems that takes in raw material from outside and makes more of itself. Patterns that persist by metabolism and reproduction are said to be alive. Life is, as such, a delicate balance of interdependent complex systems providing mutual feedback ${ }^{11}$. The whole of each structure is more than the component parts: a heart without a body will be lifeless, and the same will happen to a body without a heart.

An important part establishing this philosophical framework is the idea that reality can be reduced to information, and ultimately it might even be described as a computational universe

\footnotetext{
${ }^{10}$ Langton in Artificial Life - overview

${ }^{11}$ Lovelock in turn points out the boundaries between inorganic and organic matter being diffused and proposes Gaia as an organism resulting from the holistic interplay of feedback mechanisms formed by organic and inorganic systems on the planetary scale of the Earth (in Gaia, Medicine For an Ailing Planet).
} 
$[256]^{12}$.

The transition from organic and social processes to ones of computational nature happens by means of 'discretizing' phenomena. An anecdote describes the extraordinary moment when Descartes, laying in bed due to illness, devised a system of coordinates to map out the flight of a fly in the empty space of his room. Descartes abstracted the movement of the fly from the fly itself. ALife too argues that by reducing phenomena to discrete measurable patterns, we can abstract the processes from their embodied materiality. These can later be mapped out and recreated in distinct material forms. Take into consideration, for example, the famous flocking algorithm from Reynolds [206]. Since what is instantiated is the process of the behaviour of flocking, the algorithm might be used to represent birds. The entities in the virtual world are not birds, nor are they flying; however they are flocking.

ALife abstracts life processes from embodied biological life forms, mapping out these into processes of computational nature. Along these lines, Adami argues a duality between computation and physical dynamics, proposing that events might be physical in the computer rather than mathematical representations [8]. He gives the example of modeling a wave propagating through contiguous memory locations given that each wave has been programmed to react like a spring with a particular mass and specific amount of friction. Under these conditions it would then be possible to see an excitation propagate as a wave in the memory of the computer. This vision is shared by Steve Grand, the author of Creatures [104]. It is worth quoting him in full:

'We can therefore think in terms of different orders of simulation. Our simulation of atoms is a first-order simulation, but the molecules made from it are second-order simulations because they are made not by combining computer instructions but by combining simulated objects. What I am claiming is that secondand higher-order simulations are real things in a way that first-order are not and that these emergent entities are freed from some of the restrictions that apply to first-order ones'.

He continues:

'So when we build second-order virtual entities out of first-order, simulated building blocks, we break through from procedural space into a parallel universe - a looking-glass world. From that moment on, the things we build are more or

$\overline{12}$ 'You know, the universe is the only thing big enough to run the ultimate game of life. The only problem with the universe as a platform, though, is that it is currently running someone else's program' (Ken Karakotsios cited in Kevin Kelly, Out of Control: The New Biology of Machines. Fourth Estate, London, p.451, 1994). 
less equivalent to the things we find in the natural world, and I think they are just as real' [103].

\subsubsection{A narrative construct}

As Adami reminds us, "it is clear that one is performing "experiments", not "simulations" [8]. In the looking-glass world, a chemical is a memory location indicating a value that represents the concentration of that substance. A chemical reaction is thus a mathematical construct that converts one or more chemicals into a product. What happens when the premises to build the first-order constructs fail to capture the entire nature of the building block substance? As Cariani alerts, ultimately we are dealing with 'life-forms' and 'life-processes' which can be described by finite automata [61]. Tim Taylor argues that the parasitism seen in Tierra is not entirely of emergent nature but rather it is the direct consequence from the specific implementation of Tierrians [232]. Tierrians just happen to explore a feature of the architectural design of the software that Ray didn't think about beforehand. In CEs, there may be emergent processes resulting from self-organization of the structures. Yet, entangled with this level of naturally occurring emergence, there is a simultaneous level of behaviours directly resulting from the software design. Quoting Adami about the use of individual-based models in biology:

'On the macroscopic level, the salient characteristics of the agents have to be decided upon, and it is rather rare that these decisions are made with strict theoretical concepts in mind. More often than not, they are subject to change and tinkering. On the macroscopic level, emergent effects are (precisely because of their nature) often subjective ... a particular set of parameters may tend to be adopted as standard simply because of the subjectively interesting patterns it may produce. Thus in the language of evolutionary biology, you often "get what you select for" ' [9].

Behind the discursive superficial veil, there are symbols and manipulations of data-structures. As literary critic Katherine Hayles reminds us, 'in these representations, authorial intention, biomorphic interpretation and the program's operations are so interwoven that it is impossible to separate ... statements about the program's operation and interpretations of its meaning are in continuous interplay with each other' [108]. The difference between the material space of the computer and the imagined space appears blurred. In the imaginary space, one creature in 'heat' moves towards another. In the material space, segments of code instruct an agent that, as a consequence of the state of some variable below a certain threshold, the agent will 
adjust its variables of position to those of some other agent of which the global pattern is similar or is compatible to its own (using some type of metric). Hayles puts it clearly: it is a narrative that 'changes electric polarities on silicon into a high drama of a Darwinian struggle for survival and reproduction' [108]. Behaviours restricted to organisms appear juxtaposed with the execution of segments of code in the informational domain. Assumptions we have about natural behaviour are transported into the narrative when these two spaces collapse. She continues advocating the presence of the authorial hand in ALife: 'Analogy is not incidental or belated but central to the program's artefactual design' [108].

The work from anthropologist Stephen Helmreich deconstructing ALife practice is also critical with regards to this discussion. This author scrutinizes the primeval developments, unveiling strong re-inscriptions of culture in ALife, which appears reinforced through a discursive practice. Cultural preconceptions from the dominant western culture, such as dominant notions of gender, Neo-Liberal accounts or classic models of family appear in discourses and narratives camouflaged as a Darwinian struggle for survival [112]. To Helmreich, authors of CEs, rather than being neutral modelers of life, actually 'transport received stories into new hardware', thus they actively shape the world and its narrative [112]. If the proponents of the strong claim describe their works as 'life-as-it-could-be', Helmreich argues that these constructions 'are built from specific visions of Life-as-we-know-it' [112]. Other examples of these cultural values at play include monogamous families, or even heterosexual and productive sex widespread across models.

It takes the pragmatism of John Holland, one of the leading pioneers of ALife, and author of the celebrated Echo, to refer to computational models as maps or cartoons of life. Some features are captured, emphasized, or exaggerated, while some others are neglected or removed. As in cartooning, the skill goes into choosing what is to be emphasized and what is to be thrown away [119]. Referring to CEs as 'representations', Holland implicitly concedes that we are operating in the literary realm: the power of discourse and the identification of the audience and its practitioners with a shared common narrative. Practitioners appear, as such, as promoters of a spectacle (in Barthes terms), orchestrators of representations of life. It is, in part, this literary and metaphoric process which transports into the narrative the meaning of death or birth to squares disappearing or appearing in a simulation.

\subsection{Final notes}

This chapter lays out a summary of the fundamental ideas that inform the praxis of CEs. At the root of CE artworks is a long tradition of artistic works that have a systemic and algorith- 
mic nature. Following predecessor artworks which play with tempo and dynamics, such as in Calder's or Tinguely's, the digital construction of CEs reshapes systemic generative processes to offer an art made of dynamic and potentially ever-changing artefacts. These constructions are structured around the framework of ALife, which is fundamentally informed by Darwinian and Medelian precepts of evolution. ALife admits a bottom-up operative methodology where processes of self-organization, autopoiesis and emergence occupy a central role. The aesthetics of CEs build to a greater extent on these. 


\section{The Functioning of Computational Ecosystems}

\section{On Writing and Reading Artistic Computational Ecosystems}

'But consider the subject or agent modeled here [in swarm systems], if thats the story we want to tell: a clone in a crowd, unchanging, with no traction on the space it inhabits, existing in an ongoing, perpetual present. If these systems provide images of contemporary society then they are, at best, naive and utopian.'

Mitchell Whitelaw in System stories and Model Worlds: A critical approach to Generative Art

\subsection{Introduction}

A critical part of human culture consists of 'maps of meaning'. These are shared systems of relationships of concepts, and codes which translate these concepts into language. These codes are social constructions and result from cultural conventions. Burial stones provide some of the earliest empirical evidence of human intelligence. For early humans, there was a significant difference between these and other stones randomly found on the landscape. These stones can evoke responses from other human beings; they have inscribed meaning. By placing stones in a certain disposition and sharing amongst the members of the tribe the meaning of that construction, early humans were building something powerful, a system of representation. These stones exist as mineral matter for those who are not aware of the code, and they exist simultaneously in a virtual context for those who know it. In a leap to the other extreme of the temporal spectrum of the human story, the building stones that Evolutionary art artists use in their constructions also convey meaning. They build on existing systems of representation. 
Earlier, I approached CEs from a technical point of view, discussing the material processes and the set of ideas informing their construction. The present chapter complements these previous ones by means of looking at their functionality, and analyzing these artefacts as cultural forms. This is to understand the systems of representation at play. How is it that electronic pulses in the hardware of the computer appear at a semantic level associated with narratives of life? To assist in this project, I will articulate a number of voices (such as Latour, Klastrup, Hayles, Eco and Holland) to lay out a theoretical framework which attempts to see behind CEs' formal novelty and invention.

This present chapter looks at the processes at play when these artefacts provide context and artistic meaning. This is of critical importance for artists as well as the overall community of producers, namely for the potential use of the $\mathrm{CE}$ as an instrument for the animation of general purpose NPCs.

This discussion serves to propose an inclusive model of narrative. This model combines the agency of the population, the materiality of code, the physical artefact, the audience, and the 'interpretative framework', as proposed by Klastrup. This, I argue, confers CEs the potential to address projects which are significantly distinct from those of their aesthetic cousins: other more orthodox evolutionary art in the computational medium (such as Scott Draves's Electric Sheep [84]), or even carbon-based ecosystems based art (such as Ken Reynaldo's Hydroponic herb garden [204]).

\section{Evolutionary art and computational ecosystems}

A symbolic nature lies at the very foundation of computers. Put simply, transistors modulate electric currents to signify binary codes, which may then be transformed into humanly intelligible information by means of transducers, such as microphones, displays or loudspeakers. This symbolic architecture of codes and their respective translations scale up to inform, to a great extent, the whole mode of functioning and outcomes. EvoArt also builds on this process and establishes its roots upon a methodological approach borrowed from the classic procedure of Genetic Algorithms from Evolutionary Computation. In this technique, the syntactic elements contained in the genome are transformed into their semantic representations. In traditional EvoArt, an encoded blueprint (the genotype - Gtype) is converted to its pictorial or audible representation (the phenotype - Ptype). As discussed earlier, the complexity of this process of conversion from the Gtype to the Ptype is open to artistic creativity, and the linearity and distance involved in this process of transformation of symbols and their representation differs widely amongst artists. The diversity of the outcomes that this simple methodology entails is illustrated in Whitelaw [251]. 
One of the established ways the Gtype-Ptype paradigm has been explored is by means of applying this idea to whole populations of interacting autonomous agents in CEs [192, 225]. Computational Ecosystems are communities formed by multiple autonomous individuals, which have metabolic-like cycles, are organized in hierarchical food chains and trade amongst them units of energy. With these systems, individuals are also represented and structured by the information initially written in their Gtypes, and later this information is transformed into some form of phenotypic representation. However, in addition to this process, the autonomy of the individuals, which is so characteristic of CEs, generates an interesting dynamic of selforganization and emergence with cyclic changes of density. Tierra and Polyworld are classics established in this area in the early 1990s [257, 202]. CEs used in EvoArt explore these processes of self-organization and emergence as main mechanisms to generate heterogeneity and novelty in the works. In some cases the Gtypes are directly turned into sounds, or visualized. Wakefield and Ji, for instance, produce sounds from the transcription of the Gtypes [248]. In other cases the dynamics themselves have been deemed interesting enough to be visualized or turned audible. The soundscapes produced by Eldridge and Dorin result from the actions and status of the agents in the virtual space [90].

\section{A narrative process}

In both cases listed above, the sonification produced is an abstract process for the audience. Other instances, however are of a more explicit and representational nature, namely when they rely on visuals and represent the individuals by means of shapes or volumetric information. In Senhora da Graça [21], for instance, herbivores and carnivores have distinct physical traits which allow a visual identification as members of that particular class. In these cases, the duality between the machinic processes taking place at the levels of the hardware and software, and the respective visualizations on the display of the computer are more emphatic.

Katherine Hayles has established a discussion in this territory to argue for a gap between the processes occurring on the material level of the artefacts and the interpretative processes at the conceptual level. This gap, she suggests is filled by the narratives of ALife (discussed in the previous chapter) $[108,109]$. To understand how these processes contribute to the formation of meaning and context, and consequently how they may be instrumentalized by artists, I will take a closer look at them in the next section. For this purpose, the representational system of CEs will be examined. This project starts by laying out a discussion on its modes of representation. 


\subsection{A narrative construction}

The idea of 'maps of meaning' was mentioned earlier. To examine these shared systems of relationships of concepts, or modes of representation, it is helpful to look at the way semiotics looks at language and signification. For four decades, semiotics has been concerned with the poetics of language, the way representation produces meaning. Semiotics is the general science of signs, which studies cultural phenomena as if they were sign systems, i.e. systems of signification ${ }^{1}$.

At the core of the cultural process of meaning, two systems cohabit in close interdependence. The first is formed by a system of concepts that sets relationships of correspondence between the instances: people, objects, events. This forms a conceptual map, giving meaning to the world. The second is the correspondence between this conceptual map and a set of signs which stand for these concepts. The connection between concepts and signs is established by means of codes. When we think of a tree, it is the linguistic code which tells us to use the English word TREE [107]. Put simply, meaning is thus a result of the correlation between these three levels: the material, the conceptual and the linguistic.

\section{A semiotic reading on CEs}

Things by themselves, rarely if ever, have a fixed and permanent meaning. The production of meaning depends on processes of encoding and decoding: on interpretation. The meaning we give to things (be they objects, people or events) departs from the interpretive framework that we carry, and under which we look at them. A bag with paper cardboard may be a garbage bag or a piece of $\mathrm{art}^{2}$.

Semiotics is a key instrument in understanding CEs by the analysis it has produced on the linguistic code. From the semiotic perspective, the reader becomes as important as the writer in the process as meaning is given and taken. Interpretation occupies a central role in this process.

I will recover now a problem stated earlier in the previous chapter, where a set of mid-level instructions from an hypothetical software program forming the CE is described as:

\footnotetext{
${ }^{1}$ In linguistic studies, the semiotic approach cohabits with the discursive approach that is more concerned with the effects and consequences of representation - its politics, focusing historically and in the context in which languages are deployed. Discourses are ways of referring to or constructing knowledge about a particular topic or practice that provides ways to speak or behave in relation to a social entity. Discursive practices define what is appropriate or not in our formulation, in our practices with respect to a context [107].

${ }^{2} \mathrm{I}$ am referring to the famous incident in 2004, when a bag containing discarded paper and cardboard, part of the work of Gustav Metzger, being exhibited at Tate Britain was accidentally thrown away by a zealous cleaner.
} 
'...when the value of variable $a$ of the agent $k l$ is lesser than a certain threshold, the list $b$ is inspected to find any arbitrary agent $k 2$ whose data structure has a compatible pattern to the one of this agent. Once this other agent $k 2$ is found, then the variable $c$ of agent $k l$ must adjust its current value to that of the variable $c$ of agent $k 2$ by an $x$ amount'.

As a consequence of these interrelationships between material, conceptual and linguistic domains, this set of instructions will be described on the semantic level as 'a creature searching and finding prey to hunt because it is hungry'. Hayles stresses the active role of the viewer filling in the gaps in narrative during the transition from the material to the imaginary spaces:

'These bodies of information are not, as the expression might be taken to imply, phenotypic expressions of informational codes. Rather, the "creatures" are their codes. For them, genotype and phenotype amount to the same thing; the organism is the code, and the code is the organism. By representing them as phenotypes, visually by giving them three-dimensional bodies and verbally by calling them ancestors, parasites, and such, (...) elides the difference between behaviour, properly restricted to an organism, and executing a code, applicable to the informational domain. In the process, assumptions we have about behaviour, in particular thinking of it as independent action undertaken by purposive agents, are transported into the narrative.' [109].

Multiple semiotic signs ${ }^{3}$ conspire to make meaningful the colour change of two blue circles turning into red on the computer screen. A 'shared conceptual map' is required for this transformation of pixels changing colour into linguistic codes to be effective. It is up to interpretation to decode and read these changes on the computer screen as two creatures 'becoming enraged and fighting' in the context of the narrative of the CE. However this transformation of blue circles into signs, as living beings (or at least characters within the $\mathrm{CE}$ ), is only part of the equation and happens in a first layer of representation (which Barthes would call denotative). Stefen Helmreich argues that there is more at play in ALife. In his polemic book, Silicon Second Nature, he claims the sub-text of CEs to be made of representations of a 'neo-liberal' subject [112]. In that work he problematizes ALife, suggesting that by inscribing certain

\footnotetext{
${ }^{3}$ Saussure is one of the key figures in linguistic structuralism and semiotics. For Saussure, meaning is formed by a system of signs. At the material level, instances of the world exist as signifiers. The mental associations produced from these instances are the signified. In order to produce meaning, both signifier and signified are required to be present. It is the connection between these two levels that establishes the linguistic sign. These can be of an arbitrary nature and are established by cultural practices, 'there is no natural or inevitable link between the signifier and the signified [107].' The language system, the general rules and codes, which the users must share, is referred to as the langue.
} 
behaviours on the creatures, the signifiers, the authors are far from neutral. For instance: inscribing only productive sex on their populations, the authors of CEs are building a caricature of life. He argues that the wider cultural values that the ensemble of signs addresses are those from a restricted and specific ideology. In his point of view, these constructions rather than the proclaimed motto of ALife - 'Life-as-it-could-be' - are built from 'specific visions of Life-as-we-know-it' (italic added) [112] ${ }^{4}$.

What Helmreich is referring to in his work is the Barthesian mythic level ${ }^{5}$ : the connotative quality of the works and the messages that these convey at their sub-text. It is from this level that my argument departs. The implied social references inscribed by the designers/programmers on the behaviours of the virtual entities will play a central role in our discussion. In the next section I will discuss how, in CEs, signs formulate connotations, generating the narrative processes and contexts.

\footnotetext{
${ }^{4}$ Indeed the emphasis on predatory and sometimes parasitic relationships dominates largely the practice of artistic CEs. The narrative of the 'neo-liberal subject' identified by Helmreich is just one of the many possible narratives. Besides accounts on predation as a the basis for the theory of natural selection, others are clearly possible: narratives of sharing, narratives of interdependence, narratives of trust and altruism. Mutualistic and comensalist interactions play a role that is as important in nature as predation and parasitism. Yet these have been largely overlooked in the domain of artistic CEs. There are, of course, exceptions. Dorin, for instance, has explored how individuals benefit others, unrelated by blood or kinship, by virtue of their own existence. In Habitat and Constellation, the individual's body contributes to the formation of the conditions that are necessary for other individuals' survival $[81,82]$. This is an approach that needs to be furthered in the domain of CEs by incorporating ideas explored in game theory. Examples are the implementation of mechanisms that look at how self-preserving actions might be benefitial to others, or how altruistic behavior may also become advantageous in the mid and long term.

Niche formation is another topic underrated by the Neo-Liberal emphasis on 'survival of the fittest' based upon predatorial interactions. And yet, this property is perhaps as important in evolutionary terms as the basic set of interactions. The ability to create self-preserving artefacts that change the environment pervades nature. Beavers and their dams is just one obvious example of this phenomenon. Humans too, create complex cultural systems to change the natural order of 'survival of the fittest' by physical attributes. Nobility or secret societies, such as the Freemasons, are just some examples of such self-preserving artefacts. McCormack is one of the few authors investing in the importance of niche formation for evolution in the domain of CEs.

${ }^{5}$ The operativity of the sub-text was something addressed by Roland Barthes. A classic example is of an advertisement where a certain dress is associated with elegance. Having recognized the material as a dress and produced a sign, Barthes suggests progress to a second level, which links these signs with wider cultural subjects such as 'elegant' or 'cool'. The first and descriptive level is called by Barthes 'denotation'. The second and interpretative level is called 'connotation'. Here, signs are interpreted in terms of the wider realm of social ideology. In his work Mythologies, this author provides an illustration of representation taking place through two separate but linked processes: on the first level (the elements of the image), the signifiers and signifieds (a black man, clothing, one gesture, a flag) get united to form a sign. The symbol of this denotes a message: a black soldier is giving the French flag a salute. On the second level, this message completes the sign by linking a set of signifieds with a broad ideological theme about French colonialism. Barthes calls this second level of signification the myth.
} 


\section{A Gestalt of forces: a peculiar mode of narrative}

Departing from the previous identification of two interpretative levels (denotative and connotative) and an understanding of CEs, firstly, as representations of life permeated by structural gaps which are filled by narrative; and secondly, as open-works of which the dynamic interaction of components generates new relationships in a potentially endless evolution, we can start building a theoretical framework under which we can unfold the narrative process and formalize CEs' modes of functioning.

Material agency Flickering pixels on the screen might become 'alive' when the conceptual map of the beholder provides a context where these same pixels signify living creatures (or their caricatures). However, how do we build these conceptual maps? These pixels are in the actor-network-theory said to be actors. Bruno Latour, one of its proponents, in the second chapter of Reassembling the Social, contends a perspective of sociology where objects assume critical importance in terms of the roles they play as part of human interaction and behaviour ${ }^{6}$. The actor-network theory is useful for our discussion as it provides a material-semiotic account of interpretation, which complements Structuralist Semiotics. Objects, in that sense, are not simply the bearers of symbolic projections, but rather become actors called to intervene in enactments taking place within networks composed of humans and non-humans. Agency, for Latour, exists in different grades of causality; as he puts it: 'there are many metaphysical shades between full causality and sheer inexistence.' Objects in this context exist as actants waiting for networks where they gain figuration, when they allow, permit, afford, inform, etc.; in other words, they change a state of affairs by making a difference, and thus become actors. These webs of relations get established momentarily, and some of these actors may influence or make a difference in the course of some other actors' figuration within the network they are located [140]. Pixels on the screen, in that sense, don't have a fixed meaning. As Latour suggests, it is the context provided by the network which provides the actants their roles. As with the celebrated bag of cardboard displayed at the Tate gallery, the meaning of pixels is contextual. These may be meaningless flickering dots or living creatures in a Darwinian struggle for survival; the reading is dependent on the momentary conceptual map at play. In the context of

\footnotetext{
${ }^{6}$ Latour denies any distinction between nature and culture. The following is a verbal image used to raise this discussion:
}

'there is no doubt that you have become a couch potato in front of your tv set thanks largely to the remote control that allows you to surf from channel to channel - and yet there is no resemblance between the causes of your immobility and the portion of your action that has been carried out by an infrared signal, even though there is no question that your behaviour was permitted by the tv command' [140]. 
CEs, this map is formed by a momentary network of material and non-material actors: pixels, $3 \mathrm{~d}$ meshes, websites, the artificial intelligence used, or the theory of natural selection; they all conspire to form a fluid combination of interactions, forming a web of forces, and operating simultaneously at various levels of interpretation. In the next section I will try to identify some of these actors.

Actors in a network: The worldness of the Virtual To unveil what these main entities operating in our domain are, it is helpful to invoke a taxonomy developed by Lisbeth Klastrup to study the poetics of virtual worlds. Klastrup is an internet theorist, who in her study of the 'worldness' of these spaces, suggests an understanding of the world 'both as a fiction, a social space, a performative space and a special form of game'. In this process, she deconstructs the world into four functions: as an interpretative framework; a prop; a simulation and as a lived narrative [132]:

The world as interpretative framework, or fiction, is the shared context or story, the concept or story behind the world. It constitutes a reference point from which the actions make sense (for instance, the story of its creation and evolution, the cosmology of its' inhabitants). The interpretative framework is the first actant contributing to situate the elements in a common and shared territory, a contextual space and time [131].

The concepts of simulation and shared prop reinstate the Paideia and Ludus, these modes of play as defined by Roger Caillois [59]. These functions are proximal to gaming and performance. In 'paideia', players explore fantasy and escape reality, whereas 'ludus' involves patience in learning skills and acting in a social context. In the function of simulation, the success of the navigation and interaction of the audience in the virtual space, and with the virtual individuals, depends on his/her knowledge of rules. As a shared prop, the audience engages within the world in a make-believe attitude, embodying an avatar representation or pretending to be someone else. These functions appear to be connected, as in both situations the audience pretends to be 'in' the world, interacting with the real objects and creatures in a game environment.

Finally, the function of the world as lived narrative or story producer refers to aspects originated and experienced during the interactions with the world. The world is understood as a stage for a shared experience [132].

Refining Klastrup's work, it might be important to extend the concept of the virtual space as a lived narrative to other contributing actants critically engaged in producing the story. CEs' systems have intrinsic dynamics generated by the agency of the forms of artificial life which inhabit them: the individuals in the ecosystem. These interact autonomously and establish new 
relationships: new entities are originated in the system, others may disappear, while some others might change their shape and form. CEs' systems are dynamic and live in constant flux, and this flux (re)generates the network of signifiers, actants participating in the process of interpretation, in accord with the above framework.

With CEs, the textuality ${ }^{7}$ includes an assemblage of actants, such as the individuals in the ecosystem, the website where the world is described, as well as the processes in the rendering pipeline or the vertex of a $3 \mathrm{~d}$-surface (this will be illustrated further in one of the case studies presented, when some of these material elements are brought to the center of the narrative process). In Technosphere, for instance, when the users are selecting their creature to be either a carnivore or an herbivore, they are actively participating in the construction of a narrative process which will unfold throughout the 'lifetime' of that creature [192]. The emails the creature will 'send' a posteriori informing about who it has fought against, or eaten, are some of the signifiers which will only reinforce this dialectics.

\section{System stories: a worldness narrative}

Equipped with this framework, I will now attempt to formalize a notion of narrative in CEs. System stories is a term conveniently borrowed from Mitchell Whitelaw to describe the stories brought by artefacts made out of code ${ }^{8}$. Whitelaw suggests the notion of 'system stories' to denote 'a translation or narration of the processual structures, ontology, entities and relations in a software system'. A free-understanding of a system story is about a narrative where the system not only adds new events to the world but, in doing so, it reinvents itself. The author sets the system, the initial conditions of the story, which then unfolds autonomously, producing new interactions and relationships. These autonomous processes might, in turn, recreate the system in feedback loops, in an auto-catalytic process. The interplay of the components creates new behaviours or properties. The system might even engender behaviours which redefine the rules of the evolution of the system. The system and the emergent story become inseparable from each other. But Whitelaw's notion of system stories does not get extinguished in the system. He is also addressing the connotative level of the works:

'But consider the subject or agent modeled here [in swarm systems], if thats the story we

\footnotetext{
${ }^{7}$ In Klastrup's media-inclusive proposition, textuality is described as 'the place of the reader and text in the process of reading. The text contains signs that the reader, in the process of reading, decodes and interprets, be it graphical, auditive or verbal signs'. She continues, 'the text does not necessarily need to be written text, but can be all forms of cultural artefacts with a signifying function' [131].

${ }^{8}$ In the paper System stories and Model Worlds: A critical approach to Generative Art, Whitelaw unfolds a fierce attack on a kind of generative artwork with limited discursive practice. He calls 'weak narrative' the 'system stories' of the 'clockwork constellations' (in his words) that are classic swarms. In these, he sees a missed opportunity to engage with the more complex social and cultural systems we live in.
} 
want to tell: a clone in a crowd, unchanging, with no traction on the space it inhabits, existing in an ongoing, perpetual present. If these systems provide images of contemporary society then they are, at best, naive and utopian'. Whitelaw is interested in the stories being conveyed at the mythic level by the sub-text of these systems. As discussed earlier, to consider the subtext, we need to consider the entire contextual map, and to do so we need to scrutinize the actors forming the network of relationships. Departing from the articulation of this concept and our previous discussion, I will situate the narrative of CEs as the model of story which emerges from a system of dynamic interactions.

CEs appear, as such, as intrinsic spaces of mimesis and representation where the 'worldness' of the CE constitutes the foundational model of expression. Equating Klastrup's interpretative framework with diegetic processes, we can say that, in CEs, diegesis and mimesis appear combined $^{9}$. Echoing Hayles' gap between the material processes at the electronic level, and the interpretation of those processes, with CEs the characters rendered visible represent the internal data-structures in an apparent mimetic process; however, their 'second reality', as Caillois would put it, the realm where their actions are interpreted, is provided by the interpretative framework, the shared story where these actions make sense.

Fundamentally, CEs are defined as assemblages of material and non-material elements, artefacts dependent on their generative mechanism, but equally subordinate to their context: the interpretative framework, the shared story, the simulation, the lived narrative. Ultimately, what emerges from this picture is that, at a software level, when detached from the typical narrative of 'fox-eats-rabbit-eats-grass', the essence of the CE is as an abstract generative model for spontaneity, heterogeneity and novelty, which needs to be coupled with a constellation of other material and conceptual agents to formulate meaning.

Meaning in CEs relates material processes, linguistic signs, and conceptual frameworks. The narrative process emerges from the textuality of the CE: the product of the conjugation of the interpretative framework and the material aspects of the artefact. This includes a constellation of processes as diverse as the sounds being played, or the triangles in a 3d-mesh of the landscape, or the processes modeled in the software describing the behaviours, or the text in the website where the work is accessed, or even the quality of the display or projection; and this heterogeneity of material aspects combines in a Deleuzian mental-automaton with the viewer who accesses the work ${ }^{10}$ : his/her perceptual abilities, his/her awareness of the theory

\footnotetext{
${ }^{9}$ Diegesis is a mode of narration where a storyteller tells about the experiences of the characters. Since Plato, this form of narration has been opposed with mimesis in which the story-telling process illustrates the experiences of the characters by means of actions.

${ }^{10}$ Gilles Deleuze, in his two volumes of work dedicated to cinema, suggests the 'mental automaton', a circuit made of body, flesh and light, which is formed by the cinematographic object and the viewer. This cybernetic circuit is initiated by the sensory stimuli of the electrical pulses from the movie and the nerve signals and
} 
of Natural Selection, or his/her previous knowledge of computers. The resulting dynamics produces an ever-changing and almost fluid landscape where meaning is generated from the articulation of the material and the conceptual dimensions.

\subsection{Case studies}

I will now present two case studies where system stories are critically instrumentalized for artistic purposes. This discussion will work in conjunction with the major case-study WisLM, discussed later in chapter 7.

In the first of these works, the materiality of the virtual world was explored to convey a narrative of nostalgia and ecological-loss by means of integrating the material properties of the textures and 3d-geometries in the narrative. The second example serves as a counterexample. This is used to exaggerate and illustrate the gap between the material processes occurring in the software system and what becomes finally rendered visible to the audience. A dance performance of humanoid avatars emphasizes this gap and enhances the importance played by the interpretative framework in these artefacts.

impulses that are generated in the viewer. Once this circuit is established, the impulses no longer come from the movie but from the circuits formed by the brain, 'mixing a multitude of cinematic signs with bodies' [73]. 


\subsubsection{Senhora da Graça}

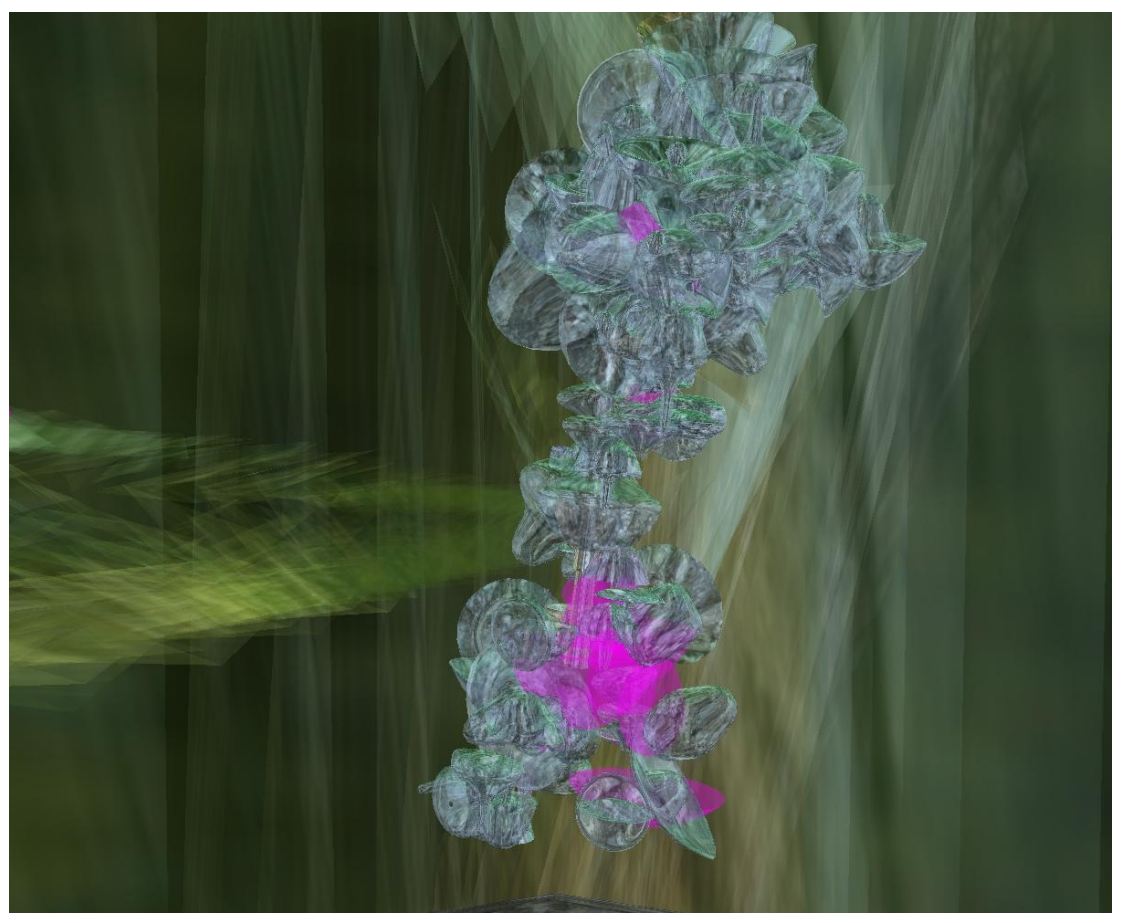

Figure 5.1: Still image from Senhora da Graça, where a population of plants, herbivores and carnivores evolves.

This section introduces the first case study for discussion to illustrate this narrative process. The artwork Senhora da Graça is a memorial in the form of a virtual ecosystem composed of clouds of rain, soil, plants, herbivores, carnivores and scavengers. Senhora da Graça denotes a valley, near Sabugal in North-East Portugal, which in 2000 was submerged to build a new dam. With the help of photographs taken at the site around 20 years ago, from which this work borrows the name, this work aimed to reference a period or moment in time. The photographs of reference are presented in a distorted and somehow abstract way when applied as $3 \mathrm{~d}$ textures on the exterior surfaces of the creatures (skin), on the soil, and in the skies of the VW. As the surfaces are not static, the generative dynamic of the interaction of creatures permanently rebuilds the world and the shapes. As a result, the photographs keep making reference to a moment in time, but the living and dynamic frames where they are applied as $3 \mathrm{~d}$ textures (the creature's bodies) evolve in time making them unrecognizable. Having lost their pictorial value as photographic objects, they retain their conceptual and chromatic values. In a metaphoric way, as happened with the submerged place of Senhora da Graça, the photographs appear unrecognizable; they are shadows of the moment they evoke. 
Accessing Senhora da Graça, a spectacle is offered, a parade of abstractions from which the first-time viewer has difficulty unraveling any meaning, if any at all. However, on the website the viewer has access to a text describing the intentions and motivations, illustrated with an introductory short story, in the same way as in an operatic performance where a libretto helps to introduce and decode the narrative, (part of) the interpretative framework.

One of the keys for our discussion resides in this libretto, specifically since it provides access to the interpretative framework. Due to its material properties (shapes, volumetric definition, transparencies, and other pictorial elements of composition) the virtual world rendered visible in Senhora da Graça has an obvious interest as an isolated sculptural and material object. But the semiotic signification is dependent on the textual narrative. This relationship is not passive and, as Latour suggests, the virtual world also prolongs, continues and expands the narrative: The introductory text finds a natural follow up in the deformation of the surfaces and the evolution of the creatures/pictures in the virtual world. In that sense, the virtual world has a dual and hybrid quality since it is only complete when in the presence of another actant, the libretto (in the form of a website), its extension. Rather than finishing with its physical boundaries, the virtual world extends and is extended with/by other contributing actants to form the mental automaton. It is this dynamic constellation that signifies and gives expression and meaning. 


\subsubsection{Vishnu's Dance of Life and Death}

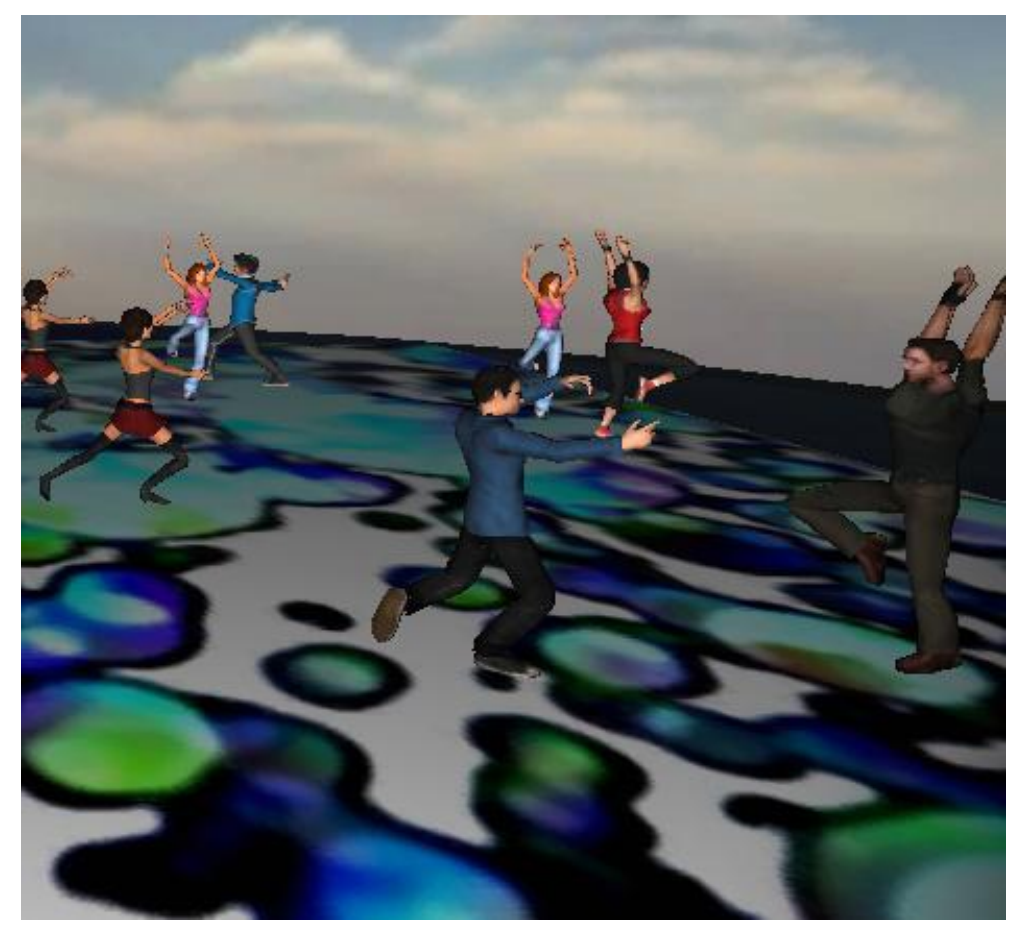

Figure 5.2: Still image from Vishnu's Dance of Life and Death, a choreography for NPCs animated with a CE.

The second case illustrates and emphasizes, almost by absurdity, the gap alluded to by Hayles in the two-levelled operativity of CEs. This work is an automated choreography performed by non-player characters in a VW. A CE functions as the main mechanism driving the avatars' movements and actions on stage. In designing Vishnu's Dance of Life and Death (from now on referred to as Vishnu's), the main motivation was that of questioning and comment on ALife with a work where an ecosystem provides context, content and dynamics in the artwork. In the Hindu mythological tradition, Vishnu is known as 'the Preserver of the universe'. While two other major Hindu gods - Brahma and Shiva - are regarded respectively as the creator and destroyer of the universe, Vishnu is the essence of all beings, the force that maintains the universe. In an exploration of the narratives of life which is part of the ALife framework, Vishnu's is a dance performance where the sequence of movements in the choreography is dictated by an algorithm, which simulates a virtual ecosystem (with a population organized in a food chain). In an ironic artistic comment on the rhetoric of life surrounding ALife practice, the deity's role of maintainer of all existences appears here inscribed in the computational algorithm. 
In Vishnu's, 3d characters perform a dance on a stage, mimicking the evolutionary course of life. The dance appears to be sometimes coordinated while at other times it seems entirely improvised. Avatars first appear from the sides of the stage and move towards a more central position, repeatedly executing the same movement. Then the choreography intensifies and the avatars play their parts with heterogeneous movements. They abandon the stage at different times, while new dancers join the performance. The sound texture is adapted from the cacophonous overture of Ligeti's Le Grand Macabre: a collage of sonorities adequate to what is seen on stage.

Since the environment consists of multiple avatars, the course of the performance is dictated by the autonomous interactions and evolution of the population. As a consequence, two different runs of the performance will provide different experiences for the audience. Sometimes it ends quickly while at other times it perdures. The dance is a visual representation of the ecosystem. The performance on stage provides a very direct visualization of the underlying multiagent state space, with a direct connection between the current state of the ecology and the configuration of the dancers on stage. Each avatar on stage is an individual belonging to the population. Each arrival to the scene is equivalent to a birth, while each exit represents a burial. Each interaction during the lifetime of an avatar is translated into a movement on stage. The algorithm establishes a correspondence between events in the ecosystem and the movements performed in the dance. With this model, the action of an avatar feeding in the virtual ecosystem might correspond to an 'arabesque', and its escape after a fight with another creature in the ecosystem's habitat might correspond to a 'jeté' on stage.

One of the immediate merits of this work is in detaching the workings of the system from the visualized traditional narratives of life the ecosystems are associated with. The visualizations are consequent to the ecosystem, but incongruous in terms of the immediate (eco) narrative. The work still draws ironically on these, but it shows that, detached from the rhetoric of life, the $\mathrm{CE}$ is ultimately a computational model for the generation of dynamic heterogeneity and apparent spontaneity in communities of NPCs. It is up to the practitioners to bring in the appropriate actants to create their narratives.

\subsection{Final notes}

This chapter attempts to lay out a theoretical space for situating and experiencing CEs as instruments for broader artistic dialogues. This journey departs with a work of deconstruction of the narrative processes in these artefacts. Hayles, Helmreich, Saussure, Barthes and Latour were invoked to discuss this mode of representation. During the course of this chapter, I 
identified the processes involved in bringing material and conceptual elements together to participate in generating a field of interpretation where meaning and messages are generated. To fill in the gap alluded to by Hayles, a series of actants (in the ANT sense) are invoked by the artists/modelers. This gap was argued as a place of artistic creativity. The term 'system stories' was proposed as a name for this model of narrative.

The first case study, Senhora da Graça, assisted with the formulation of this model of narrative by means of emphasizing the material processes, when these are instrumentalized in contextual and interpretative processes. This notion is suggested as a critical conceptual tool in understanding these works. A system story correlates the material properties with the context in which the CE exists, as the semantic elements actively participating in the narrative process. This textual construction goes on to include a wide network of actants. This goes from the language of code used in the implementation of the virtual system, to the story read by the audience, or even its processes of diffusion. This construction is dynamic and non-stop. Due to its open nature, works evolve in time when new relations and interactions unfold and get established within this network.

Following this, the dissociation of the operative mechanics of the system from the narratives of life was illustrated. To assist in this process of separation from the narrative and operative levels, I brought into the discussion the work Vishnu's, an artefact where these two levels appear disjointed and somehow play on absurdity. This gives us the opportunity to emphasize and argue the $\mathrm{CE}$ as a generative model for novelty and heterogeneity in communities of general purpose NPCs. This separation provides a gap which offers fertile territory to explore narratively.

To conclude, writing and reading these artefacts entails a complex articulation of material processes and conceptual ideas. CEs are generative instruments for heterogeneity and spontaneity. System stories are the narratives emergent from these dynamics, and these are critical in the processes of authorship and interpretation. Messages are conveyed when artists model the material processes producing the CE; and when they invoke other actants: first to build the diegesis, the interpretative framework, and then to bridge the gap between this and the material artefact. 


\section{A Comparative Study Towards Persistent Computational Ecosystems}

\subsection{Introduction}

This chapter concerns the problems affecting these systems, and in particular one that constrains the free implementation of these systems in VWs: the persistence of the population. Earlier in the Literature Review (chapter 2), the need for such a study was evidentially established. Authors, such as Gordon Selly [194] and Tom Barbalet [33], gave voice to what is an understated issue impacting these systems, and widely neglected in technical papers reporting the implementations. These authors recall the unpleasant consequences of the wild fluctuations in population density when their systems went public and then collapsed. Noticeably, extinction and overpopulation are phenomena impacting natural ecosystems (Figure 2.3) and have been studied for a long time in ecological science. Conservation biology identifies this problem as stochastic extinction in small-populations [63]. Small populations are those potentially offering greater risk when their numbers fluctuate. For instance, it does not take a large number of consecutive deaths to significantly lower the population density of a small community. This is a problem with, as yet, no known solution in the field of ecological simulations.

Due to the limited rendering and processing capabilities, the community of NPCs in WisLM is one such example of a small population. Of course, there may be a number of technical standards to circumvent this limitation, such as the subdivision of the virtual environment into small sections, which are treated separately. However, I wanted to take this unique opportunity to address the problem in a straight forward way. Throughout this chapter, some methodologies for the construction of persistent CEs are examined. By persistent, I mean systems where the population numbers fluctuate but do not tend to reach either: i) extinction levels, or ii) exponential growth. Overpopulation and mass-extinction are two well-known problems of these 
systems and different mitigation strategies are analyzed: a) adding or removing individuals when the system is running near extinction or overpopulation respectively; and b) inhibiting insertion or removal for the same conditions. The efficiency of these strategies is compared in terms of minimizing the interference with the natural evolutionary course of the community. This efficiency is measured by how well the algorithms lower the number of artificial operations (forced births/deaths, or inhibition of these) that are required to maintain a sustainable and steady density of population.

\subsection{Characterization of the problem}

Systems consisting of multiple individuals can be implemented relatively easily by means of simple single-size tournament-selection models [71]. These require fitness comparisons to be performed amongst the individuals in the population, with some individuals being removed from the pool while others are allowed to procreate, thus filling these empty demographic slots. The result is an exploration of the pool of Gtypes known as genetic drifts. Sometimes, however, for one reason or another - in particular by design specifications originated in artistic scripts - the model needs to show fluctuations in demographics. On the other hand, the model may also need the individuals in the population to be autonomous, for instance when it simulates heterogeneity of behaviour and spatiality of movements. Despite a more complex implementation, CEs offer these and other advantages. Due to the agency of the individuals forming the community, these systems are also characterized by the emergence of niches or potentially complex ecological interactions, such as mutualism or parasitism.

These features significantly widen the palette available for creative users. For example, the artists and scientists Alan Dorin and Jon McCormack explore these peculiarities; Dorin in a figurative artwork on epidemics and the spread of diseases [78] and McCormack, in an interactive audiovisual installation where the entities call to attract visitors to their proximity [161].

Existing models differ in a large number of properties, particularly in terms of their spatial organization and representation, or the agency models that are implemented on the individuals (Appendix-A). However, one fundamental premise informing the design of these systems is the notion that each individual has to capture and store energy, and then convert this into useful work. In this context, trophic interactions are defined when groups of similar individuals interact and exchange energy with other groups in what can be described as predator-prey interactions. This permanent need to trade a currency (the units of energy) provides an intrinsic motivation for agency, which becomes a central aspect of the conceptualization and system 
design.

In this section, I will identify specific groups or patterns of communities in existing CEs, which are organized based on patterns of energetic exchange. The reason for performing such a categorization is that there may be issues which are specific to particular types of dynamics. In particular, certain communities may prove to be more prone to extinction than others. In our case, I will focus on population Ht which was the organization of the system for WisLM.

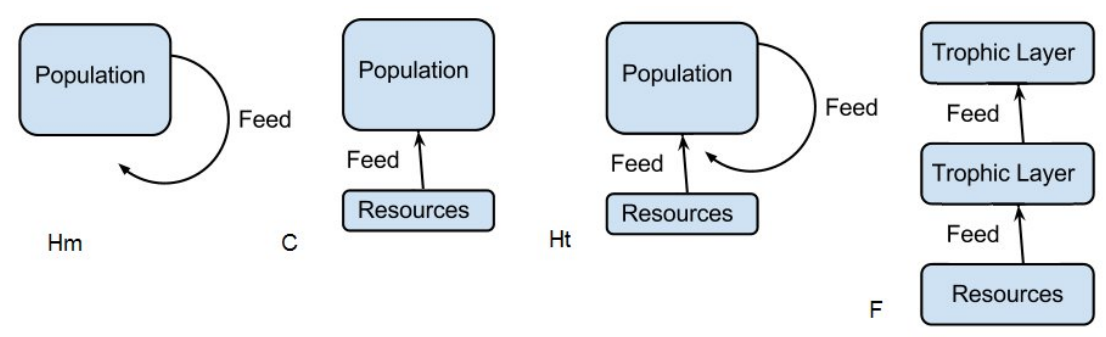

Figure 6.1: Four distinct models of population, from left to right: Homogeneous $(\mathrm{Hm})$; Chemostat $(\mathrm{C})$; Heterogeneous $(\mathrm{Ht})$; and Food web (F).

\subsubsection{Homogeneous model $(\mathrm{Hm})$}

The simplest model is the one using homogeneous populations (Figure 6.1-Hm). Interactions are established within a closed community of similar individuals. This is the model used, for instance, in A-Volve [225]. In order to survive in the virtual tank of $A$-Volve, fish need to capture other fish coinhabiting in the tank. In this type of community the interactions are mainly pre-established during the design stage of production, but they may also be designed in order to evolve with time, generating dynamic food webs which shrink or expand with the emergent complexity of the interactions.

\subsubsection{Chemostat model (C)}

In chemostats, an external input feeds a constant flow of resources to a population of cells. Populations of type $\mathrm{C}$ can be characterized as having a sub-population of consumers distinguished from its source of energy. This might be a set of 'food-bits' or a sub-population of producers.

These two non-interbreeding groups are usually represented using distinct data-structures. In Genepool [244], for instance, dynamic individuals feed on passive 'food-bits'. In general, the subpopulation of consumers is used to model populations of heterotrophic individuals, such as carnivores, that mostly rely on other organisms to survive. The source of energy is used 
to simulate autotrophs, such as green plants, which can manufacture food from their abiotic environment. Tierra [202], GenePool [244] and Polyworld [257] are classic works which employ this type of design. In GenePool and Polyworld, the source of energy is constituted by passive abiotic units, which appear as if by magic on the surface of the simulation. Tierra is a special case where individuals compete for units of processing time.

Extended derivations from this model creatively play with the flow of energetic input. In Life Spacies II [228], or BlackScholes [74], the appearance and growth of producers is not constant and infinite, as in the previous examples, but rather is dependent on some actions external to the community. These are performed either by the public in the installation space, as in the case of Eden [158] and Life Spacies II [228], or by some other external factors, such as movements of shares on the Stock Exchange, as in BlackScholes [74], or even variations in the weather as in Garden of Chances [124].

\subsubsection{Heterogeneous model $(\mathrm{Ht})$}

This model is a combination of the previous two. It describes works where individuals in the population feed, not only from an external source but also from other members of their community. This is the type of interaction at play in xTNZ [19] and Senhora da Graça [21]. In these CEs, individuals have dietary constraints emulating some form of chemical metabolism, which restricts the range of possible prey. These metabolic constraints force the emergence of multilayer food webs [213] where some individuals are able to exclusively prey on producers, while others are able to prey not only on producers but also on other consumers, whereas some other individuals are only able to prey on other consumers. This is the model I will be focusing on during the present inquiry as this is the type of community in the second version of WisLM.

\subsubsection{Food web model (F)}

In contrast to the emergent food webs previously described in Technosphere [192] or Eidea [173], individual metabolisms and trophic interactions are pre-established. In these projects, the ecosystem is typically composed of three different trophic levels that emulate carnivores, herbivores and plants. Here, evolutionary forces do not change individual diets as happens with some of the previous models (such as $\mathrm{Hm}$ and $\mathrm{Ht}$ ).

Some commercial games, drawing on evolution, make use of this model of community. The procedural creatures of Spore, for instance, have 'metabolic' capabilities and scope predefined [26]. The user can only choose where his/her creature will fit in the rigid hierarchy of the food chain. 


\begin{tabular}{|c|c|c|c|c|c|c|c|}
\hline \multirow[t]{2}{*}{ NAME } & \multirow[t]{2}{*}{ TYPE } & \multicolumn{2}{|c|}{ REPRESENTATION } & \multirow[t]{2}{*}{ INFINITE/FINITE RESOURCES } & \multirow{2}{*}{$\begin{array}{l}\text { ACTIVE/PASSI } \\
\text { RESOURCES }\end{array}$} & \multicolumn{2}{|c|}{ FORCED ACTIONS } \\
\hline & & DEPTH & DESCRIPTION & & & LOWER THRESHOLD & UPPER THRESHOLD \\
\hline Avida & $\mathrm{C}$ & $2 \mathrm{~d}$ & Grid (Infinite) & Can be configured & Passive & No specification & Configurable up to 12.000 \\
\hline $\begin{array}{c}\text { Artificial } \\
\text { Nature [248] }\end{array}$ & $\mathrm{Hm}$ & $3 \mathrm{~d}$ & Torus & Finite & Active & $\begin{array}{l}\text { New seed organisms are triggered } \\
\text { when the population falls below a } \\
\text { threshold }\end{array}$ & $\begin{array}{l}\text { Less food is supplied and more } \\
\text { energy lost to entropy when near a } \\
\text { superior limit }\end{array}$ \\
\hline $\begin{array}{c}\text { BlackScholes } \\
\text { [74] }\end{array}$ & $\mathrm{C}$ & $2.5 \mathrm{D}$ & Sphere & $\begin{array}{l}\text { Activity of Stock Exchange feeds } \\
\text { the planet. Potentially is finite, but } \\
\text { in practice it infinite }\end{array}$ & Passive & $\begin{array}{l}\text { A fixed number of genetically } \\
\text { distinct populations were } \\
\text { maintained artificially above a } \\
\text { certain number of individuals }\end{array}$ & No handling of overpopulation \\
\hline $\begin{array}{l}\text { Colourfield } \\
\text { [91] }\end{array}$ & $\mathrm{C}$ & $2 \mathrm{~d}$ & Grid & Infinite & Passive & No specification & No specification \\
\hline $\begin{array}{c}\text { Cosmos } \\
{[232]}\end{array}$ & $\mathrm{C}$ & 1D & $\begin{array}{l}\text { Discrete line } \\
\text { of cells }\end{array}$ & $\begin{array}{l}\text { Infinite. CPU time is used as a } \\
\text { commodity }\end{array}$ & Passive & No handling of extinctions. & $\begin{array}{l}\text { When full amount of memory is } \\
\text { reached, the cells with less energy } \\
\text { are removed }\end{array}$ \\
\hline Echo & $\mathrm{C}$ & $2 \mathrm{~d}$ & Grid & Regenerative resources (Infinite) & Passive & No specification & No specification \\
\hline Eden [159] & F & $2 \mathrm{~d}$ & $\begin{array}{l}\text { Grid where } \\
\text { multiple } \\
\text { agents can } \\
\text { occupy a } \\
\text { single cell. }\end{array}$ & $\begin{array}{l}\text { Audience encourages the growth of } \\
\text { biomass. Biomass has seasonal } \\
\text { variations and different types of } \\
\text { absorption of the constant 'radiant' } \\
\text { energy }\end{array}$ & Semi-active & $\begin{array}{l}\text { In the case of complete extinction, } \\
\text { the world is reseeded with new } \\
\text { creatures (randomly initialized) }\end{array}$ & $\begin{array}{l}\text { If the population becomes very } \\
\text { large, there is a limit set on new } \\
\text { creatures being born }\end{array}$ \\
\hline Eidea [173] & $\mathrm{F}$ & $3 \mathrm{~d}$ & & Finite & Passive & No specification & No specification \\
\hline FramSticks & $\mathrm{C}$ & $3 \mathrm{~d}$ & Grid & Can be configured & Passive & Can be configured. & Can be configured \\
\hline $\begin{array}{c}\text { Filterscape } \\
\text { [90] }\end{array}$ & $\mathrm{C}$ & $2 \mathrm{~d}$ & Grid & $\begin{array}{l}\text { Resources are supplied externally } \\
\text { at a constant rate }\end{array}$ & Passive & $\begin{array}{l}\text { No handling of extinctions. } \\
\text { Though: "there were some } \\
\text { extinctions" }\end{array}$ & $\begin{array}{l}\text { Fluctuations can occur up to a limit } \\
\qquad(40)\end{array}$ \\
\hline
\end{tabular}

Table 6.1: Summary of the main features of the surveyed ecosystems. From left to right, the columns indicate: the name of the $\mathrm{CE}$; the type of community this implements $(\mathrm{Hm}, \mathrm{C}, \mathrm{Ht}, \mathrm{F})$; the kind of visual representation and spacial configuration of the environment ( $2 \mathrm{~d}, 2.5 \mathrm{~d}, 3 \mathrm{~d}$ and Grid, Sphere, Torus); if resources are limited (finite or infinite); if resources have agency, or are simple 'dummies' (active, semiactive, passive); what types of interventions are performed to avoid extinction and overpopulation. 


\begin{tabular}{|c|c|c|c|c|c|c|c|}
\hline \multirow[t]{2}{*}{ NAME } & \multirow[t]{2}{*}{ TYPE } & \multicolumn{2}{|c|}{ REPRESENTATION } & \multirow{2}{*}{$\begin{array}{l}\text { INFINITE/FINITE } \\
\text { RESOURCES }\end{array}$} & \multirow{2}{*}{$\begin{array}{l}\text { ACtive/Pass } \\
\text { RESOurces }\end{array}$} & \multicolumn{2}{|c|}{ FORCED ACTIONS } \\
\hline & & DEPTH & DESCRIPTION & & & LOWER THRESHOLD & UPPER THRESHOLD \\
\hline $\begin{array}{c}\text { Garden of } \\
\text { Chances } \\
\text { [124] }\end{array}$ & $\mathrm{C}$ & $2 \mathrm{~d}$ & Grid & $\begin{array}{l}\text { Weather feeds plants. } \\
\text { Potentially is finite, but } \\
\text { in practice it is infinite. }\end{array}$ & Passive & No specification & No specification \\
\hline $\begin{array}{c}\text { Life Spacies } \\
\text { II [227] }\end{array}$ & $\mathrm{C}$ & $3 \mathrm{~d}$ & Grid & $\begin{array}{l}\text { Finite. Users control } \\
\text { the energy supply } \\
\text { entering the system by } \\
\text { way of text messages. }\end{array}$ & Passive & No specification & No specification \\
\hline $\begin{array}{c}\text { Genepool } \\
\text { [244] }\end{array}$ & $\mathrm{C}$ & $2 \mathrm{~d}$ & $\begin{array}{c}\text { Disk shaped } \\
\text { grid }\end{array}$ & $\begin{array}{l}\text { Infinite. "New food } \\
\text { bits appear at random } \\
\text { positions ... at regular } \\
\text { intervals" }\end{array}$ & Passive & $\begin{array}{l}\text { No mechanism was implemented: } \\
\text { "the population count is initialized } \\
\text { to } 1000 \text {... the animation inevitably } \\
\text { drops this number as the system } \\
\text { stabilizes... after this ... animations } \\
\text { can be watched for hours". }\end{array}$ & No specification \\
\hline $\begin{array}{l}\text { Pandemic } \\
{[78,83]}\end{array}$ & $\mathrm{C}$ & $2 \mathrm{~d}$ & Torus & Infinite & Passive & $\begin{array}{l}\text { If the population falls below a } \\
\text { threshold, two agents from the } \\
\text { population are randomly picked to } \\
\text { mate together. }\end{array}$ & $\begin{array}{l}\text { If a birth would cause an overflow } \\
\text { on the available memory, new } \\
\text { births are refused. Following the } \\
\text { unsuccessful request, a random } \\
\text { member of the population may (or } \\
\text { may not) be eliminated to make } \\
\text { room for future requests. }\end{array}$ \\
\hline $\begin{array}{c}\text { Polyworld } \\
\text { [257] }\end{array}$ & $\mathrm{C}$ & $3 \mathrm{~d}$ & Grid & $\begin{array}{l}\text { Infinite. "Food is } \\
\text { grown at a specifiable } \\
\text { rate up to a specified } \\
\text { maximum number of } \\
\text { grown food items. }\end{array}$ & Passive & $\begin{array}{l}\text { In old versions, there were three } \\
\text { possible modes: A) Random - } \\
\text { Introduce an agent with a random } \\
\text { genome B) Fitness-based - } \\
\text { Introduce an agent which is the } \\
\text { offspring of two agents chosen } \\
\text { from the list of fittest agents so far } \\
\text { C) Elite - Re-introduce the fittest } \\
\text { agent ever. Currently a global } \\
\text { multiplier is used for energy } \\
\text { consumption. By the time a } \\
\text { population reaches the } \\
\text { user-specified minimum, agents are } \\
\text { using zero energy to survive, thus } \\
\text { they cannot die due to energy loss }\end{array}$ & $\begin{array}{l}\text { In old versions, there were diverse } \\
\text { possible answers: eliminate births, } \\
\text { eliminate an agent to give space to } \\
\text { the new birth. This elimination can } \\
\text { be based on two criteria: the least } \\
\text { fit and random. } \\
\text { Current versions use a energy } \\
\text { consumption multiplier, so when } \\
\text { the population becomes closer to } \\
\text { the maximum agents are } \\
\text { consuming very large amounts of } \\
\text { energy }\end{array}$ \\
\hline
\end{tabular}

Table 6.2: Continuation from Table 6.1 


\subsubsection{Other forms of organization}

The notion of spatial organization also determines fundamental distinctions amongst the works. Communities can be considered to be spatially homogeneous when individuals are uniformly distributed in space; when they are all at the same average distance from each other. The same can be said about their resources. Conversely, a community can be spatially heterogeneous when individuals are positioned in a scattered or spatially disperse way. The type of global behaviour provided by the first type of model is more easily captured by mathematical representations. The second, however, adds extra levels of complexity. For instance, at lower densities individuals will find more difficulty finding mates, their vulnerability to predators will be increased and they will be more exposed to inbreeding depression ${ }^{1}$. On the other hand, the notion of space provides for the formation of niches of isolated groups of individuals, with all the associated possibilities that these 'bubbles' bring in evolutionary terms. When it comes to space, these computational systems may be organized in $1 \mathrm{~d}$ [232], 2d [244] or $3 d$ [192] ${ }^{2}$.

CEs may also diverge in the way individual features are defined. For instance, one important feature that contributes to niche formation is the range of the individuals' spatial perception. The individual interactions can be considered to be discrete or stochastic, depending on whether metabolic needs, or reproduction and consumption rates, are fixed or diverge amongst the population. These may be constant to all populations of a certain type, or they may change and adapt through each individual life cycle, perhaps with learning involved. Adaptation plays an important role in many models (e.g Polyworld [257] or Eden [158]), with sophisticated artificial intelligence techniques, such as reinforcement learning being used in models of agency equipping the individuals. Again, the complexity of the individuality has many gradients of complexity and differs wildly amongst models.

Each of these factors adds to the complexity of the system, and it is known from ecology studies that any kind of additional stochasticity involves an increase in the amplitude of the population fluctuations and thus in the risk of extinction and overpopulation [182]. Shaffer identifies four sources of uncertainty to which a population may be subject in biological systems: demographic stochasticity, which arises from chance events in the survival and reproductive success; environmental stochasticity, which is due to temporal variation of habit parameters and the population of competitors, predators, parasites and diseases; natural catastrophes, such as floods, fires, etc. which may occur at random intervals; and genetic

\footnotetext{
${ }^{1}$ The Alee effect describes the impact of density factors in the growth of species. It suggests that aggregation can improve the survival rate of individuals, and that cooperation may be crucial in the overall evolution of social structure.

${ }^{2}$ To be more precise, we would need to refer to these works as operating on $4 \mathrm{~d}$ since these are fundamentally structured upon time.
} 
stochasticity, resulting from changes in gene frequencies due to the founder effect, random fixation, or inbreeding [216]. The many levels of uncertainty increase the difficulty of the task of calculating the risk of extinction or overpopulation. The difficulty of precisely capturing these variations is voiced by Shaffer:

'There are several theoretical models for predicting the probability that a small population will go extinct and the time this will take. There are also numerous models of population growth under stochastic conditions (demographic, environmental, or both). But these models either embody unrealistic assumptions or lead to unresolved mathematical problems [216]'.

Results in the area are approximations rather than precise laws [182]. These difficulties of risk assessment in natural habitats are mirrored in the complexity of CEs. The idiosyncratic nature of the models and the great variability inherent to ecological interaction probably preclude direct applications of any single theoretical model to all generalized scenarios.

\subsection{Methodology}

\subsubsection{Questions}

Remember that the initial problem was motivated by the necessity of having an artwork on public display for continuous periods of seven hours per day over a period of two weeks. This artwork is characterized as a CE and the aim was to investigate which strategies are best with regard to minimizing the number of interventions required to maintain the system running continuously without crashing due to overpopulation, or becoming deserted due to extinction. My aim is to analyze some mitigation strategies by comparing Inhibitive vs Active methods and Prediction vs Rescue modes of intervention.

For this study, a Monte Carlo simulation was produced with a population of type Hm. This was the population model of the first version of WisLM presented to the public. In the second version (colonials and servants - consumers and producers), the population model is Ht. Yet, because interbreeding is possible in the system and additionally, as one class of individuals can produce members of the other, the classes in this last version of the work are not closed. As such, the principles for persistence of populations $\mathrm{Hm}$ are replicable to WisLM. 


\subsubsection{Architecture}

An initial closed population was assumed with no default emigration or immigration. Extraordinary insertions or removals are restricted to the ones generated by the mitigation strategies described below. The actions performed by the individuals in the world are generated from a probabilistic distribution. A set of constants describe the probability assigned to each of the 15 possible actions that one individual might perform at any particular moment in time. These are originated in the model at play in WisLM (explained in the following chapters), and are as follows: reproduce, eat, attack, move away, move, play, wander, collide, rotate, look for mate, mate, hungry, hungry for other consumers, hungry for producers. Their probabilistic values have been calculated by averaging 10 runs of the original system. These values do not change throughout the duration of the trial and are generalized for the whole population. At each cycle of the clock, all the individuals currently present in the community update and perform their current action based on this predefined probability.

Additionally, a variable describes the individuals' current energetic level. An energetic cost is associated with every possible action that an individual might perform. These costs are also predefined, static and apply to the whole population. For each action that an individual performs, a portion of its energy is used. Only by eating will the individual be allowed to regain more energy. When this value reaches zero, the individual is then removed from the community as if it had died. New individuals are added to the community when individuals trigger the respective reproductive action.

This simple model offers the advantage of abstracting the particular implementation of agency and the respective associated degrees of freedom, and thus isolates the problem of the population number under scrutiny. Simultaneously, the desired patterns of behaviour can be easily calibrated. On the other hand, we lose an important feature of agent-based models, which is the significant uncertainty associated with niche formation and the adaptation of species to one another and to their surrounding environment.

\subsubsection{The sub-models}

With the above model, the constants used for input of the system were calibrated in order to generate two different sub-types of population type Hm: a) in the first of these, the numbers drop systematically to zero (Figure 6.2-a), b) while on the second sub-model the population density grows exponentially (Figure 6.2-b). These create distinct patterns where permanent forces push the population towards the identified problematic zones of density that I want to investigate: areas of extinction and overpopulation. 

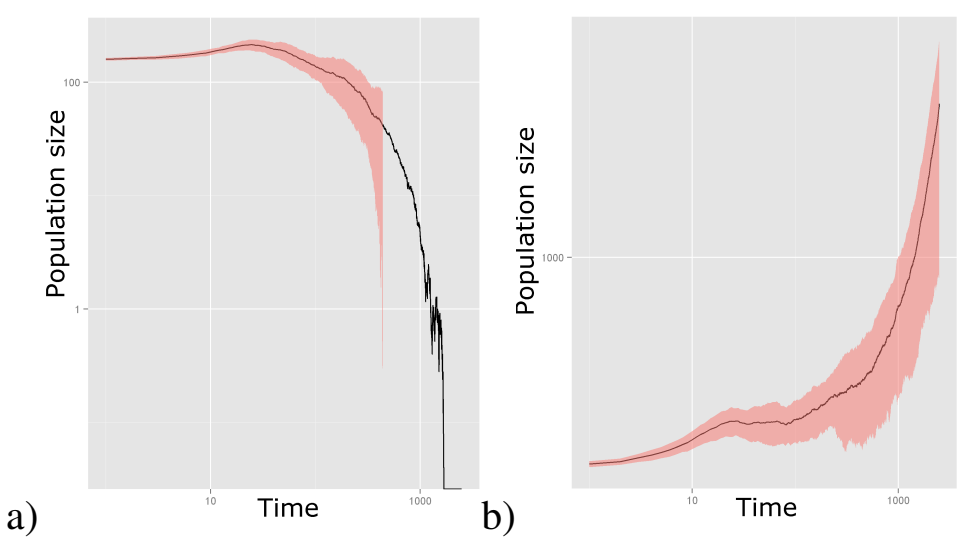

Figure 6.2: From left to right: a) 25 runs with model pushing the demographics down to extinction, and b) 25 runs with model pushing the demographics up to overpopulation.

\subsubsection{Experiments}

\subsubsection{Algorithms}

Four mitigation strategies were developed in this study in order to address the aspects of overpopulation and extinction. These strategies, or algorithms, either promote active changes in the density by adding or removing individuals in the community or, on the contrary, their strategy is more passive in the sense that they inhibit expected changes from being produced. I wanted to also analyse the impact when these changes are produced: i) as actions taken $a$ priori, as a consequence of the prediction of future densities, or ii) as rescuing measures, to recover the system from potential critical zones.

I - Rescue approaches: Two thresholds were specified, between which the population is at a level considered stable. A minimum of 50 simultaneous individuals was considered the minimum deemed empirically sufficient to maintain audience interest. This is also the minimum effective population required to maintain short-term fitness as suggested by Franklin (i.e to prevent serious in-breeding and its deleterious effects) $[98]^{3}$. As a superior threshold, or

\footnotetext{
${ }^{3}$ The rule $50 / 500$ is a rule of thumb advanced by Franklin, and prescribes 50 adults able to reproduce as the minimum value for effective population size in the short term, and 500 in the long term. The first of these values is to avoid an unacceptable rate of inbreeding and the latter to maintain the overall genetic variability required to cope with environmental changes. However, it is important to have in mind that the concepts of effective population size and census population size are distinct. Variables, such as breeding cycles, sex ratio, mating strategy, life span and historic population size all need to be taken into account when it comes to calculate the effective population size.
} 
carrying capacity, a value of 200 individuals was set since the navigation in the system would become jerky above that level.

\section{a) Active algorithm (ACT):}

The first strategy under analysis is the active insertion or removal of critical individuals when the population numbers reach one of the two thresholds (Figure 6.3-Left). Individuals are forcibly inserted when the numbers are below the lower threshold. By contrast, above the superior threshold, the oldest individuals in the population are removed from the community.

\section{b) Inhibition algorithm (INH)}

The second strategy is passive in the sense that the actions taken are inhibitory. Changes of density are prohibited when these critical thresholds are reached (Figure 6.3-Right). Above the maximum threshold, insertions are inhibited. Below the lower threshold, death events are equally inhibited.

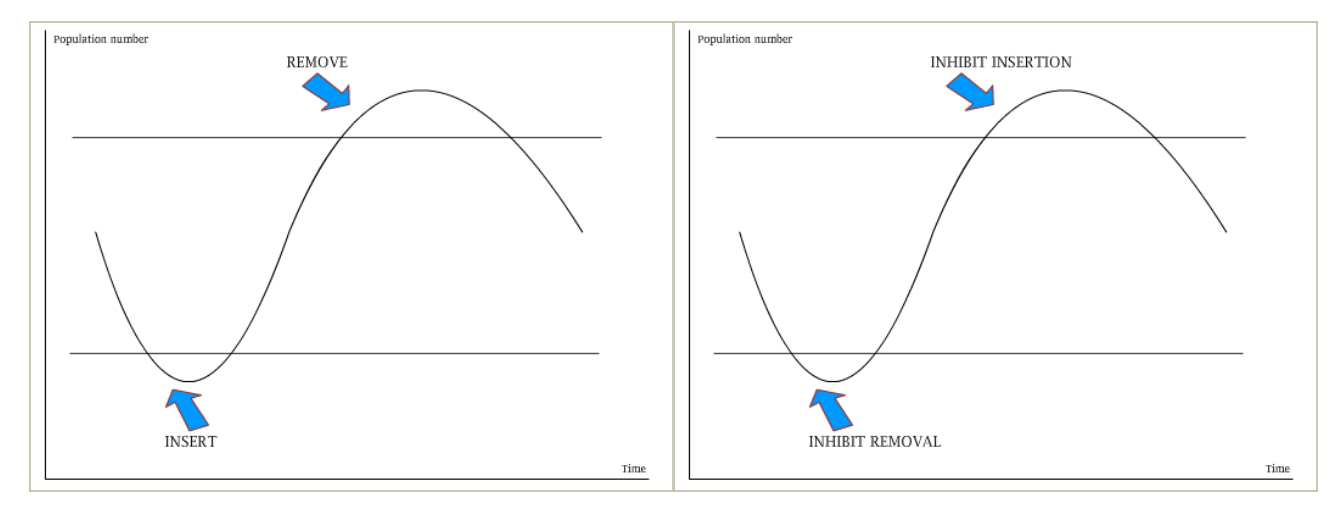

Figure 6.3: Left: Active algorithm (ACT): Individuals are forcibly inserted when the population numbers are below the lower threshold. By contrast, whenever the population goes above the superior threshold, the individuals with less energy are removed. Right: Passive algorithm (INH): Insertions are forbidden above the carrying capacity. Removals are equally inhibited below the minimum threshold.

II - Predictive approaches: The second main approach makes use of estimations about future demographics. This approach draws on the Verhulst model of population dynamics, also known as the logistic equation. This is a simple mechanistic model that describes the continuous, discrete and intrinsic growth rate $r$ of a population of size $N$ with carrying capacity $K$. This can be written as an ordinary differential equation: 


$$
\frac{d_{N}}{d_{t}}=r N\left(\frac{K-N}{K}\right)
$$

This provides a very general description of population dynamics, and consequently may be somewhat oversimplified. This simple model assumes that all members of the population have the same growth rate, and that this is constant throughout the individual's lifetime. It neglects the influence of exogenous factors of stochasticity on growth rates, such as heterogeneous populations (age, sex, etc.), environments (obstacles, temperature, etc.) as well as density effects (concentration or dispersion in space), and competition for resources. However, this model offers a simple approach to the problem of monitoring the global dynamics of the population. In this study, this was expanded to take into account phenomena of stochastic demographics by means of a local time frame to estimate the growth rate. Small populations, such as the ones analyzed here, are known to be highly sensitive to stochastic variations in demographic rates. Population growth is not perfectly continuous and it does not take many sequential deaths to drive a small population to extinction. For instance, a hypothetical sequence of deaths (D) and births (B) might be ...BDBBBD.... A second and more problematic sequence might happen in a continuous ...BBBBDD... In both cases, however, the birth rate is twice the size of the death rate. In order to integrate this variability into our model and to estimate the future size, I have added an indicator of the demographic direction to the equation. This was motivated by strategies of locomotion and collision detection used in animation, which employ a local vector of velocity pointing towards the desired direction [206]. This density direction (or growth tendency) is given by the local growth rate $r_{t}$, the growth exhibited by the system during the last interval of time $(t-1)$. At regular intervals, the system verifies how many inhabitants $N t$ exist. The local (momentary) growth rate $r_{t}$ is given by the number of births $B_{t}$ that occurred per capita $(B t / N t)$ in that particular interval subtracted by the number of local deaths $D_{t}$ per capita $(D t / N t)$.

$$
r_{t}=\frac{B_{t}}{N_{t}}-\frac{D_{t}}{N_{t}}
$$

The tendency $T_{t}$ is then described by the product of the local growth $r_{t}$ by the local number of inhabitants $N_{(t-1)}$, Eq. 6.3.

$$
T_{t}=N_{t-1} * r_{t-1} *\left(\frac{K-N_{t-1}}{K}\right)
$$

The final estimation of density $N_{t+1}$ is then given by the existing population $N$ added of this local growth tendency (Eq. 6.4). 


$$
N_{t+1}=N_{t}+T_{t}
$$

Based on these estimations, the response of the system is generated using one of the two strategies described above for the rescue approaches: Active (PACT) or Passive (PINH).
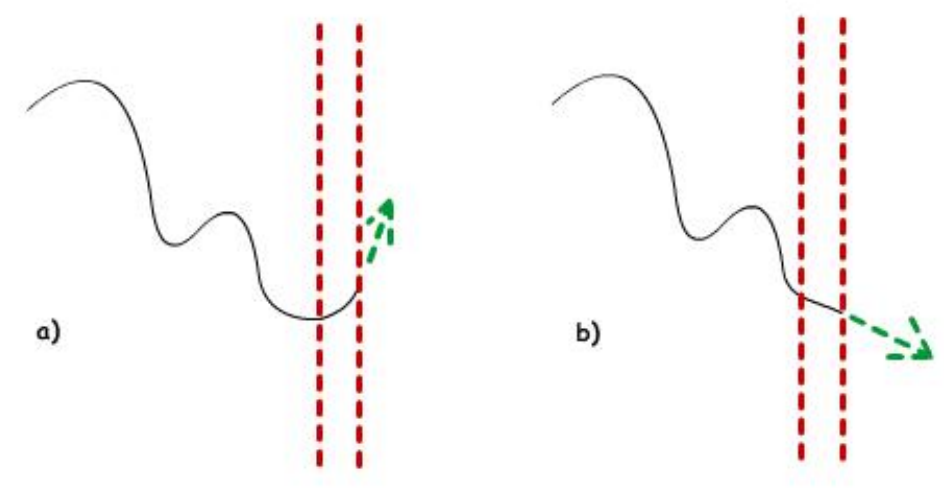

Figure 6.4: With predictive method, the estimations are based on the local tendency of growth. In the example on the left, the local growth indicates a growth, whereas on the right a decrease in density is predicted.

Summary: Two types of mitigation strategies will be examined. ACT actively intervenes in the community, adding or removing individuals when critical densities are reached. INH inhibits scheduled insertions or deletions from being performed. PACT and PINH denote methods that use the above strategies, but the values of density considered are of estimations of future densities rather than the actual.

\subsubsection{Duration and dimension of the trials}

The system was set to run for a duration of 100, 1000, 10000 and 100000 cycles, and for 50 times in each category. The temporal scale, using a power of 10 scale, provided the means for capturing different moments of the maturity of the community. The number of runs was observed to be empirically sufficient to provide consistent patterns of behaviour. At the end of each run, a portrait of the variables of the system was captured. This was to measure the degree of artificiality introduced as result of the mitigation strategies on the natural evolution of the communities. This artificiality was given by the number of 'forced actions' performed on each trial. A 'forced action' refers to the arbitrary interventions in the system outside of the 
evolutionary course strictly dictated by the interactions of the individuals in the community, such as: a) keeping an individual alive to avoid an extinction; b) populating the system with a forced insertion of an individual with random features; c) a forced removal of an arbitrary individual due to a carrying capacity being reached; or d) inhibition of new insertions due to overpopulation. The criticality of such measures is expressed by the impact these changes may potentially have on the evolutionary course of the community. This might be more or less relevant depending on the nature of the work. For instance, the inhibition of reproduction of the fittest individuals has a direct impact on the evolutionary trajectory of the community.

\subsubsection{Sensitivity measurements}

Some additional questions emerged during the experiments. One of these is relative to the magnitude of changes produced in any intervention. When the method requires active changes such as insertions or removals, is there any difference if we add/remove one or many individuals? This can be postulated as: are smaller changes better than larger? In an attempt to answer this question, the system was run again for the active methods (ACT and PACT). Only this time, rather than adding/removing 1 individual at a time, whenever the system is required to intervene, 20 individuals are added/removed (10\% of the carrying capacity).

With regard to predictive methods, there was also a variable that required closer inspection. This was the dimension of the interval used to capture the growth tendency. For predictive methods (PACT and PINH), different runs using intervals of 10 and 50 cycles were compared.

\subsection{Results}

As briefly mentioned earlier, trials were conducted within the domain of population $\mathrm{Hm}$ as this was the population in our original system. The model was calibrated to produce populations that naturally converge: a) to extinction, or b) to overpopulation. On each sub-system, the methods described above were applied and 50 different runs were performed for each sub-system/method. The variable under scrutiny is forced actions, the number of changes introduced by the system. This was measured at different moments of time for each strategy/community.

\subsubsection{Presentation of the results}

The results are presented in Appendix-B (Summary of the Trials), in a table that depicts the overall perspective of the performance. Results are presented by their mean value and associ- 
ated variance.

The performance of the algorithms is illustrated by Figures 6.5, 6.6, 6.7 and 6.8. These Figures show the number of artificial actions produced using each of methods under scrutiny. Graphs are grouped by the duration (number of cycles) of the trials.
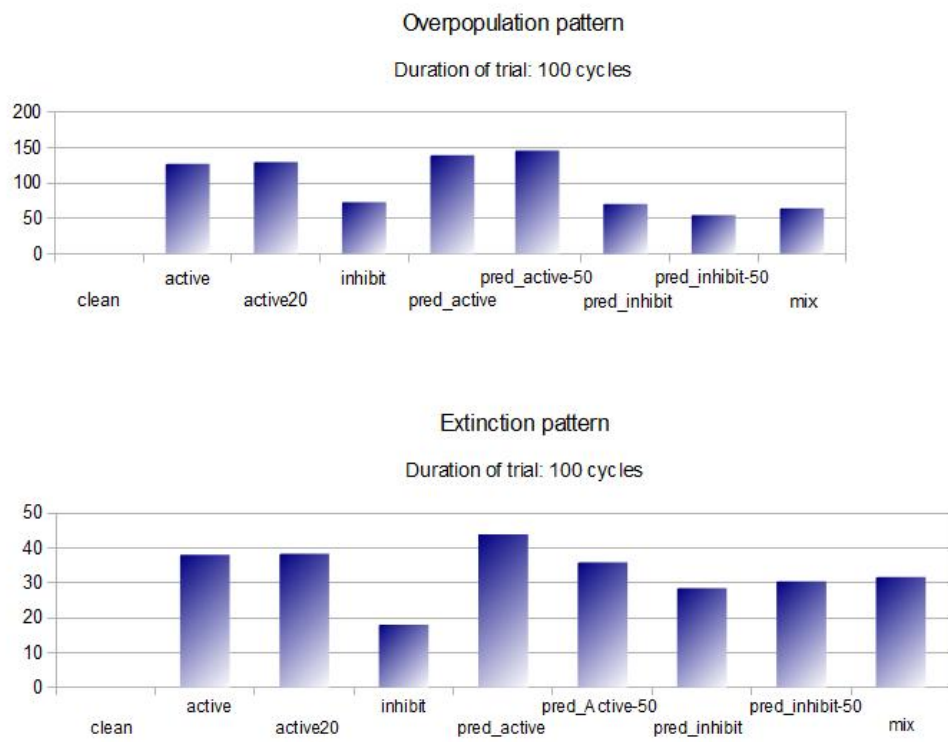

Figure 6.5: The mean (from 50 runs) of the number of artificial actions produced using each of the methods under scrutiny after 100 cycles. From top to bottom: a) populations with strong intrinsic pressure towards overpopulation, and b) populations with strong intrinsic pressure towards extinction. 


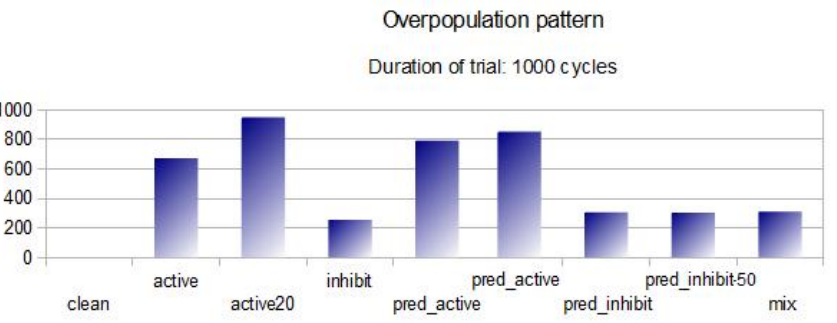

Extinction pattern

Duration of trial: 1000 cycles

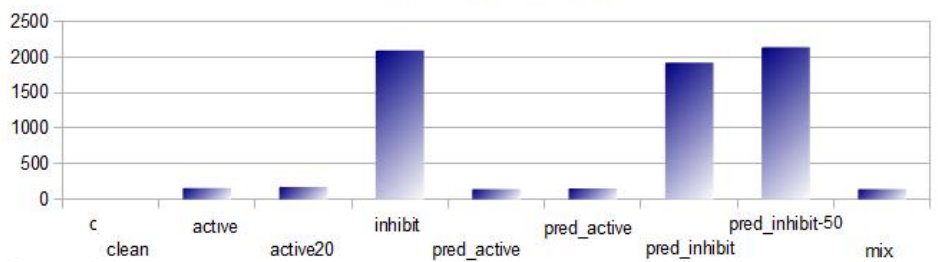

Figure 6.6: The mean (from 50 runs) of the number of artificial actions produced using each of the methods under scrutiny after 1.000 cycles. From top to bottom: a) populations with strong intrinsic pressure towards overpopulation, and b) populations with strong intrinsic pressure towards extinction.
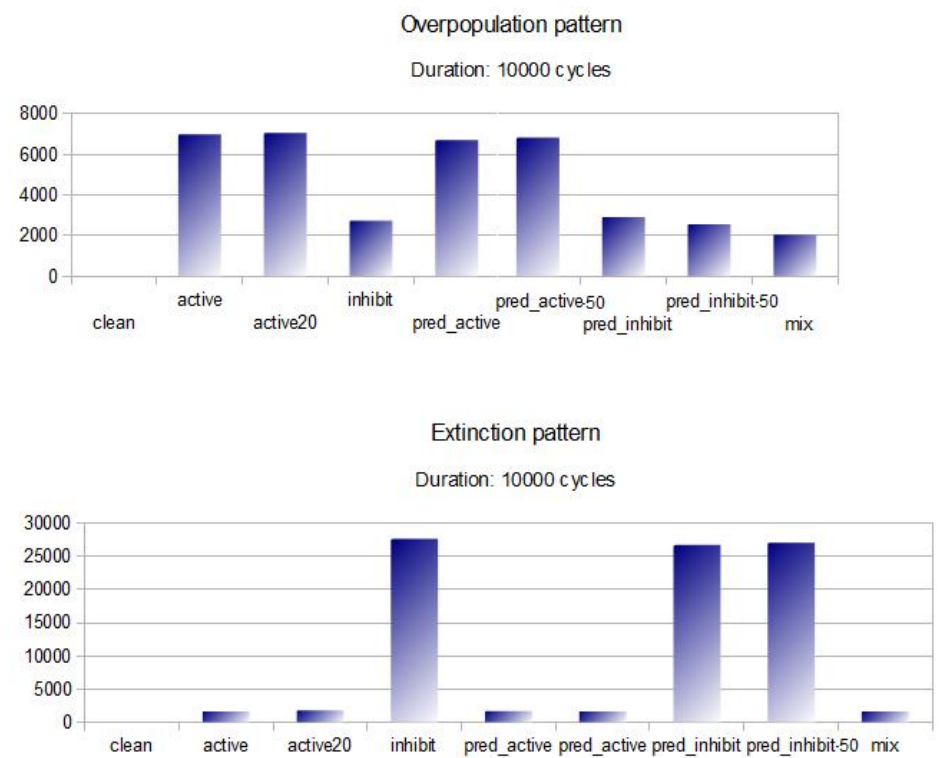

Figure 6.7: The mean (from 50 runs) of the number of artificial actions produced using each of the methods under scrutiny after 10.000 cycles. From top to bottom: a) populations with strong intrinsic pressure towards overpopulation, and b) populations with strong intrinsic pressure towards extinction. 

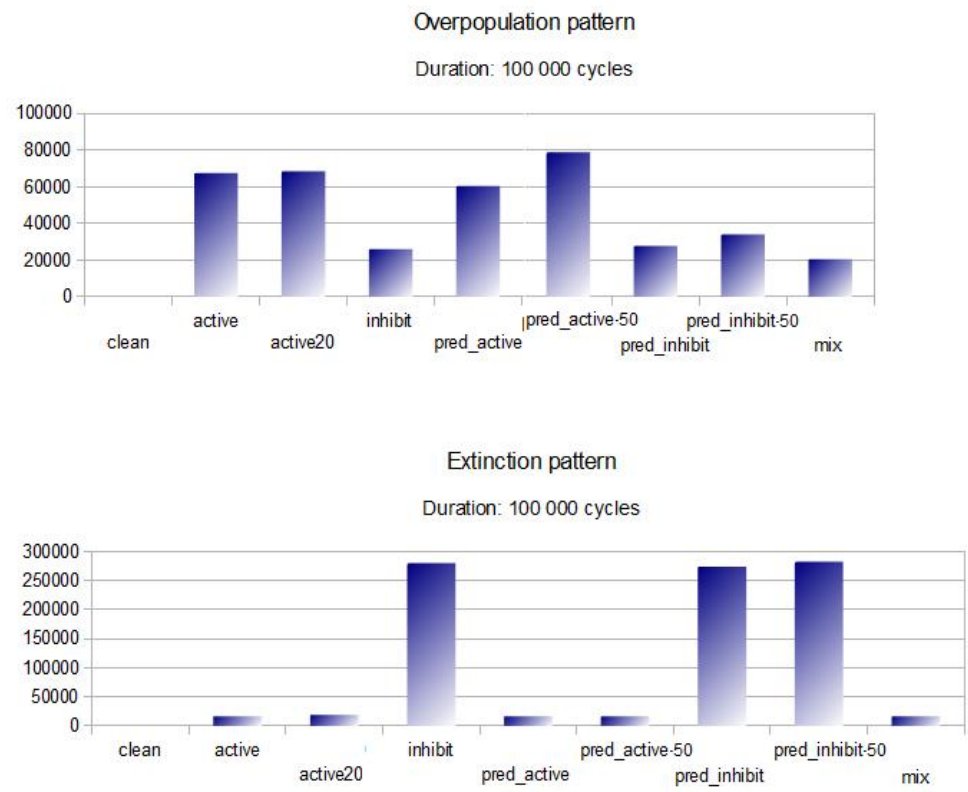

Figure 6.8: The mean (from 50 runs) of the number of artificial actions produced using each of the methods under scrutiny after 100.000 cycles. From top to bottom: a) populations with strong intrinsic pressure towards overpopulation, and b) populations with strong intrinsic pressure towards extinction.

\subsubsection{Comparison of approaches and methods}

The table presented in Appendix-B was filtered in order to emphasize the relevant data to answer the questions under analysis. Tables 6.3 and 6.4 show the comparison of the performance of the algorithms alongside, using a TukeyHSD test with a confidence interval of $95 \%$. TukeyHSD (Honestly Significant Difference) is a statistical test generally used to find which means are significantly different from one another [171]. The test compares the means of every trial with the means of every other trial; that is, for all sets of pairwise comparisons, it identifies where the difference between two means is greater than the standard error would be expected to allow. 


\begin{tabular}{ll|c|c|c} 
Duration & Method & ACT1-INH & PACT10-PINH10 & ACT20-ACT1 \\
\hline $\mathbf{1 0 0}$ & OVR & o-X & o-X & - \\
$\mathbf{1 0 0}$ & EXT & - & - & - \\
\hline $\mathbf{1 0 0 0}$ & OVR & o-X & o-X & o-X \\
$\mathbf{1 0 0 0}$ & EXT & X-o & X-o & - \\
\hline $\mathbf{1 0 0 0 0}$ & OVR & o-X & o-X & - \\
$\mathbf{1 0 0 0 0}$ & EXT & X-o & X-o & - \\
\hline $\mathbf{1 0 0 0 0 0}$ & OVR & o-X & o-X & - \\
$\mathbf{1 0 0 0 0 0}$ & EXT & X-o & X-o & -
\end{tabular}

Table 6.3: The Table shows the comparison of the active and inhibitory strategies using TukeyHSD. Test results are for the number of Artificial actions performed, with a 95\% interval of confidence. Based on the TukeyHSD test, if the difference between each pair of algorithms is significant, the pairs are marked. When any cell is ocupied with ' $X-o$ ' this indicates that the first algorithm is significantly better; conversely, ' $o-X$ ' indicates that the second algorithm is significantly better; finally, '- ' indicates the existence of no statistical significant difference between the two algorithms.

ACT1 stands for active method (removing and inserting 1 individual at a time); ACT20 stands for active method (removing and inserting 20 individuals at a time); INH stands for inhibitory method (prohibiting programmed removals or insertions); PACT10 stands for insertion and removal with predictions based on an interval of 10 cycles; and PINH10 stands for inhibitions based on predictions estimated with an interval of 10 cycles.

\section{Question 1: Is an active approach better that an inhibitory one? As mentioned} earlier, in this context, better means minimizing the number of artificial actions produced during the run. Bear in mind that an inhibitory approach offers a number of intrinsic problems since by default it changes the evolutionary course whenever it inhibits the reproduction of the 'fittest' individuals in the community.

The performance of methods using the active approach (ACT1, INH) was compared to the methods using the inhibitory approach (PACT10, PINH10) (Table 6.3). In this context, when methods are active, it is also important to examine the magnitude of the intervention. ACT1 was compared to ACT20 since the first adds/removes 1 individual at a time and the latter makes significant changes to the population by adding or removing 20 individuals each time the system is called to intervene.

\subsubsection{Question 2: Is the predictive strategy better than the rescue ones?}

The type of response from the algorithms was also analysed: when algorithms exert a preventive action a priori, based on estimations for future densities (predictive), and in contrast, when they respond to critical situations that have already happened (rescue). To assist with 
answering this second question, predictive methods (PACT1, PINH10) were compared with rescue ones (ACT1, INH) (Table 6.4). Here too, there are some questions arising as a result of the rules of implementation that were followed. Does the interval of the prediction significantly change the performance of the algorithm? To answer this last question, the methods at play were compared when short (PACT10, PINH10) and long intervals (PACT50, PINH50) are used for the calculation of the estimations.

\begin{tabular}{|c|c|c|c|c|}
\hline \multirow[b]{2}{*}{ Duration } & \multicolumn{3}{|c|}{$\begin{array}{c}\text { ACTIVE strategy vs } \\
\text { ACTIVE strategy with } \\
\text { prediction }\end{array}$} & \\
\hline & Method & $\begin{array}{l}\text { ACT1 } v s \\
\text { PACT10 }\end{array}$ & $\begin{array}{l}\text { ACT1 } v s \\
\text { PACT50 }\end{array}$ & $\begin{array}{c}\text { PACT10 vs } \\
\text { PACT50 }\end{array}$ \\
\hline 100 & OVR & - & - & - \\
\hline 100 & EXT & - & - & - \\
\hline 1000 & OVR & - & - & - \\
\hline 1000 & EXT & - & - & - \\
\hline 10000 & OVR & - & - & - \\
\hline 10000 & EXT & - & - & - \\
\hline 100000 & OVR & $\mathrm{o}-\mathrm{X}$ & $\mathrm{X}-\mathrm{O}$ & $\mathrm{X}-\mathrm{O}$ \\
\hline 100000 & EXT & - & - & - \\
\hline
\end{tabular}

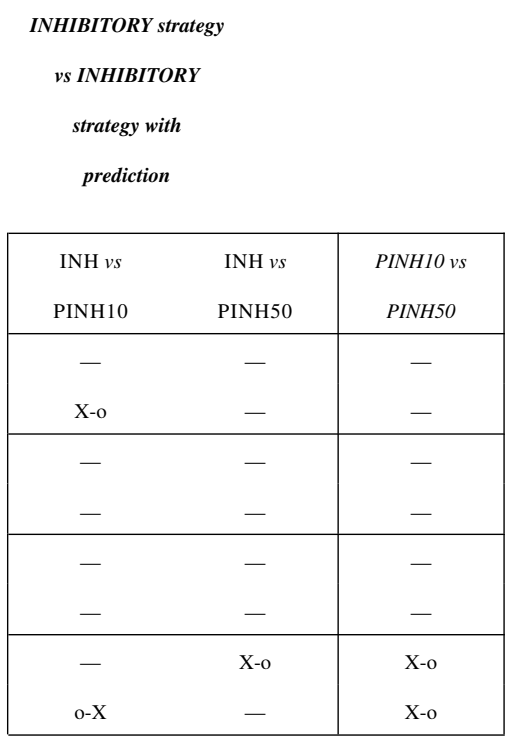

Table 6.4: The table shows the comparison of standard and predictive strategies, based on TukeyHSD. Test results are for the number of Artificial actions perfomed, with a 95\% interval of confidence. Based on the TukeyHSD test, if the difference between each pair of algorithms is significant, the pairs are marked. When any cell is ocupied with ' $X-o$ ', this indicates that the first algorithm is significantly better; conversely, ' $o-X$ ' indicates that the second algorithm is significantly better; finally, '- ' indicates the existence of no statistical significant difference between the two algorithms.

ACT1 stands for active method (removing and inserting 1 individual at a time); INH stands for inhibitory method (prohibiting programmed remotions or insertions); PACT10 stands for insertion and removal with predictions based on an interval of 10 cycles; PACT50 stands for active insertion and removal based on predictions made with an interval of 50 cycles; PINH10 stands for inhibitory strategy based on predictions estimated with an interval of 10 cycles; and PINH50 stands for inhibitory strategy based on predictions estimated with an interval of 10 cycles.

\subsubsection{Discussion of the results}

Q1: The first strategy analyzed here, ACT, intervenes actively in the communities, inserting and removing individuals when population numbers become critical. Conversely, the second 
strategy, INH, is passive in the sense that, in contrast to the previous approach, it does not insert or delete individuals to maintain the population level within tractable boundaries. Rather, its action is inhibitory, not allowing certain insertions or removals to be performed. Furthermore, the analysis was based on the impact of changes being produced as a last minute response to critical numbers (methods ACT and INH), or when these changes are produced as a result of predictions of future densities (PACT and PINH).

The outlook provided in the previous section and in Appendix-B suggests that strategies to manage population size, far from being neutral, do induce significant changes in the natural evolutionary course of the communities. Table 6.3 seems to clearly indicate that the first question analysed, comparing active and inhibitory approaches, has no clear deterministic answer, and the performance is dependent on the context. Based on the Tukey analysis, we can say that interventive methods (actively adding and removing individuals) such as ACT and PACT, perform significantly better than inhibitory strategies (inhibiting the addition or removal of individuals) such as INH and PINH. However, this result only applies to communities where the dominant behaviour is EXT (tendentially pushing the density to extinction). This result confirms the intuition, since below the minimum viable threshold ACT adds a new individual, with normal energetic levels, whereas INH keeps alive one which energy level is close to zero. On the contrary, when the behaviours of the population push the demographics towards overpopulation, inhibitory strategies perform significantly better. Here, the impact of the energy level is the opposite. Because INH maintains the individuals with the lower energetic level, due to natural death the population will decrease faster than with ACT. Remember that ACT allows the birth of the new individuals but removes one from the current pool as a compensatory measure.

On the other hand, I could not extract conclusions with absolute confidence about the size of interventions by the mitigation algorithms using an active strategy. Results indicate some benefit in making minimal interventions. However, the Tukey test is not entirely conclusive about the performance of ACT1 vs ACT20 (inserting/removing 1 versus 20 individual at a time).

Q2: Table 6.4 illustrates Tukey's result for the second question under analysis. This concerns the comparison of rescue (ACT and INH) and predictive (PACT and PINH) strategies. In addition, the former methods were analysed using different intervals to calculate the estimations (of 10 and 50 cycles). Results are inconclusive for the overall duration of the trials. Yet, in the longer runs (100.000 cycles), PACT10 has performed more efficiently than ACT and PACT50. This result questions the strength of the predictive method developed, as longer 
estimation windows should provide better results. However, the predictive method PACT10 clearly performed better than a simple ACT. Regarding the inhibitory methods, the magnitude of the results between the methods INH, PINH10 and PINH50 needs to be taken into account since they are quite similar (Figure 6.8), which means that results are consistently better in some methods but do not introduce a large difference in the final result to be considered significant. Yet, again, predictions with a shorter window lead to more efficient results for overpopulation and extinction.

Summary: What emerges from this analysis is that, from the mitigation strategies proposed, there is no single and simplified approach which covers all the critical circumstances of population density. The performance of the methods changes depending on the overall behaviour of the population, namely if this puts it at risk of extinction or overpopulation. It seems that a method which combines approaches would be much more promising. Overall, active methods, ACT and PACT, are better than the inhibitory methods, INH and PINH, for populations with a dominant pattern of behaviour EXT. Thus, actively adding and removing individuals from the community when numbers reach a minimum seems, in general, to be the best strategy to follow if we want to minimize the number of interventions to avoid extinction. However, by constrast, when populations have a strong pressure towards overpopulation, inhibitory answers are more appropriate.

A new method combining approaches (MIX): As a result, it seemed logical to combine the methods. Thus, a new methodology was designed. This time, ACT was combined with INH; the first method to deal with the minimum threshold and the second to deal with the carrying capacity. MIX, the name given to this combined response, actively inserts a new individual for every death occurring, when the population is closer to the lower threshold. Additionally, MIX inhibits births when the density is above the carrying capacity. Results of the runs can also be found in Appendix-B. The comparison between MIX and the other methods is summarized in Table 6.5 and presented in full in Appendix-B. Results are conclusive and promising. This combination of methods substantially reduces the number of interventions when compared to any of the methods described earlier in isolation. Globally, as can be seen from Appendix-B, in a majority of situations the combined method MIX has performed significantly better. For the remaining situations, there is no significant difference to the other methods, and thus it can be concluded that it is positive to use MIX as a global generalized strategy to minimize the number of forced actions. 


\begin{tabular}{|c|c|c|c|}
\hline & & $\begin{array}{c}\text { Mixed strategy vs } \\
\text { Active }\end{array}$ & $\begin{array}{c}\text { Mixed strategy vs } \\
\text { Inhibitory }\end{array}$ \\
\hline Duration & Method & $\begin{array}{l}\text { MIX } v s \\
\text { ACT1 }\end{array}$ & MIX $v s$ INH \\
\hline 100 & OVR & $X-o$ & - \\
\hline 100 & EXT & - & - \\
\hline 1000 & OVR & $\mathrm{X}-\mathrm{o}$ & - \\
\hline 1000 & EXT & - & $\mathrm{X}-\mathrm{o}$ \\
\hline 10000 & OVR & $\mathrm{X}-\mathrm{o}$ & - \\
\hline 10000 & EXT & - & $\mathrm{X}-\mathrm{o}$ \\
\hline 100000 & OVR & X-o & X-o \\
\hline 100000 & EXT & - & $\mathrm{X}-\mathrm{o}$ \\
\hline
\end{tabular}

Table 6.5: TukeyHSD Test results for Artificial actions, with a 95\% interval of confidence. Based on the TukeyHSD Test, if the difference between each pair of algorithms is significant, the pairs are marked. When any cell is ocupied with ' $X$ - $o$ ' this indicates that the first algorithm is significantly better; conversely, ' $O-X$ ' indicates that the second algorithm is significantly better; finally, '-' indicates the existence of no statistical significant difference between the two algorithms.

Despite these results, it must be said that none of these methods actually respects the full integrity of the evolutionary course of the community where they are implemented. However, if our goal is to maintain a persistent, dynamic, and diverse community, the above articulation of strategies (MIX) is likely to offer a robust model for monitoring a community. Again, we have to keep in mind that these methods do not respect the full integrity of the evolutionary course of the systems where they are implemented.

\subsubsection{Final notes}

When the goal is optimization, the most popular evolutionary algorithms have a fixed size. The variable sizes of population are advantageous when we want to explore a larger area of search space, or when we want to focus on (exploit) a particular part of this space. Meanwhile, when the goal is the simulation of some model of biology, sociology, or life in general, then the model should reflect the reality and the characteristics we want to see represented.

However, there may be other contexts where stable demographics are required in the simulation, or because the $\mathrm{CE}$ is facing a public audience which finds it to be 'boring' and 'uninteresting' when there is only a small number of individuals in the population, or any other context in which the authors may need more control over the demographics. This study is focused on this latter group of users requiring a steady flow of population; for instance, in 
complex studies where single-fixed densities are not the best solution and death and density fluctuations are aspects to factor in, but they are not at the core of the analysis. Investigations on the spread of 'memes', or the diffusion of infectious not lethal diseases, are some of these, to name but a few.

Other aspects that are tangent to this topic and worthy of investigation are the impact in evolutionary terms of the heterogeneity and diversity of decisions, such as inserting an individual with random features versus inserting an individual with features taken from the existing pool. The same kind of inquiry would be interesting regarding the impact of removing the oldest individual, or the youngest, or the one with less energy or even one at random. Studies on the area of performance of genetic algorithms examine some of these aspects. As this would open another line of inquiry, and was irrelevant to the work in question, attention was restricted to density issues.

\subsection{Conclusions}

If we envisage Virtual Worlds inhabited by non-player characters forming ecosystems, we need to somehow address the problem of the stability of these systems to avoid problems, such as mass extinction and overpopulation. What I am pursuing is a heuristic that would allow us to maintain the persistence of the ecosystem with selective and minimal interventions in the communities. In this chapter, a study was presented on the persistence of CEs by means of monitoring their performance and selective interventions. In particular, this study aimed to address alternative procedures to tackle the problem known in conservation ecology as stochastic extinction in small populations (which also applies to CEs). Two main approaches and two main methods were compared. These approaches consider the total number of inhabitants without distinguishing between species. The first method actively inserts and removes individuals, when needed, into and from the community. In contrast, the second method only inhibits certain insertions and removals from being performed. The two approaches are distinguished by the fact that the first intervenes as a response to events whereas the second intervenes as a consequence of estimations of built scenarios.

This study has demonstrated that we can indeed control the stability and persistence of the community by actively intervening in the population. The proposed method MIX combines the two methods and minimizes the number of interventions required to maintain the system in stable levels. However, there is a price to pay for this. Far from being neutral, algorithms that address the persistence of systems introduce changes and dimensions which compromise the natural evolutionary course of the communities. Future studies must examine other types 
of communities and ecological interactions. Moreover, it is important to investigate what happens when the minimum viable number of individuals and the carrying capacity change. 


\section{Where is Lourenço Marques? - I}

\section{Motivations for the Work}

'Marco enters a city; he sees someone in a square living a life or an instant that could be his; he could now be in that man's place, if he had stopped in time, long ago; or if, long ago, at a crossroads, instead of taking one road he had taken the opposite one, and after long wandering he had come to be in the place of that man in that square. By now, from that real or hypothetical past of his, he is excluded; he cannot stop; he must go on to another city, where another of his pasts awaits him, or something perhaps that had been a possible future of his and is now someone's present. Futures not achieved are only branches of the past: dead branches.'

Italo Calvino in Invisible cities

\subsection{Introduction}

This chapter describes the motivations for the artwork WisLM. This artwork is dedicated to the former city of Lourenço Marques, which since the independence of Mozambique has become known as Maputo. In the landscape of colonial Portugal, Lourenço Marques was the proud capital of the district of Moçambique (Mozambique). This work consecrates those who lived and inhabited that place and time. During the process of the decolonization of Mozambique, thousands of people were forced to suddenly abandon that country. More than the possessions and material goods, experiences of entire lives and generations were left behind. The great majority have never returned, nor do they intend to. For some, only a great nostalgia and disenchantment is left; while some others evoke the previous time as a magical space and time; others, like myself, don't have any memory of the place. I was born there, but moved away in my early days as an infant, with the rest of my family during the events described, to grow up in a small village in the north of Portugal, in a small purpose-made 'camp' to receive people expatriated from the former-colonies: the 'bairro dos retornados'. WisLM, the work presented here (available at http://www.lourencomarques.net), aims to represent 
that place from the memories of those who have experienced Lourenço Marques and had to abandon the city in the three year period from 1974 to 1976.

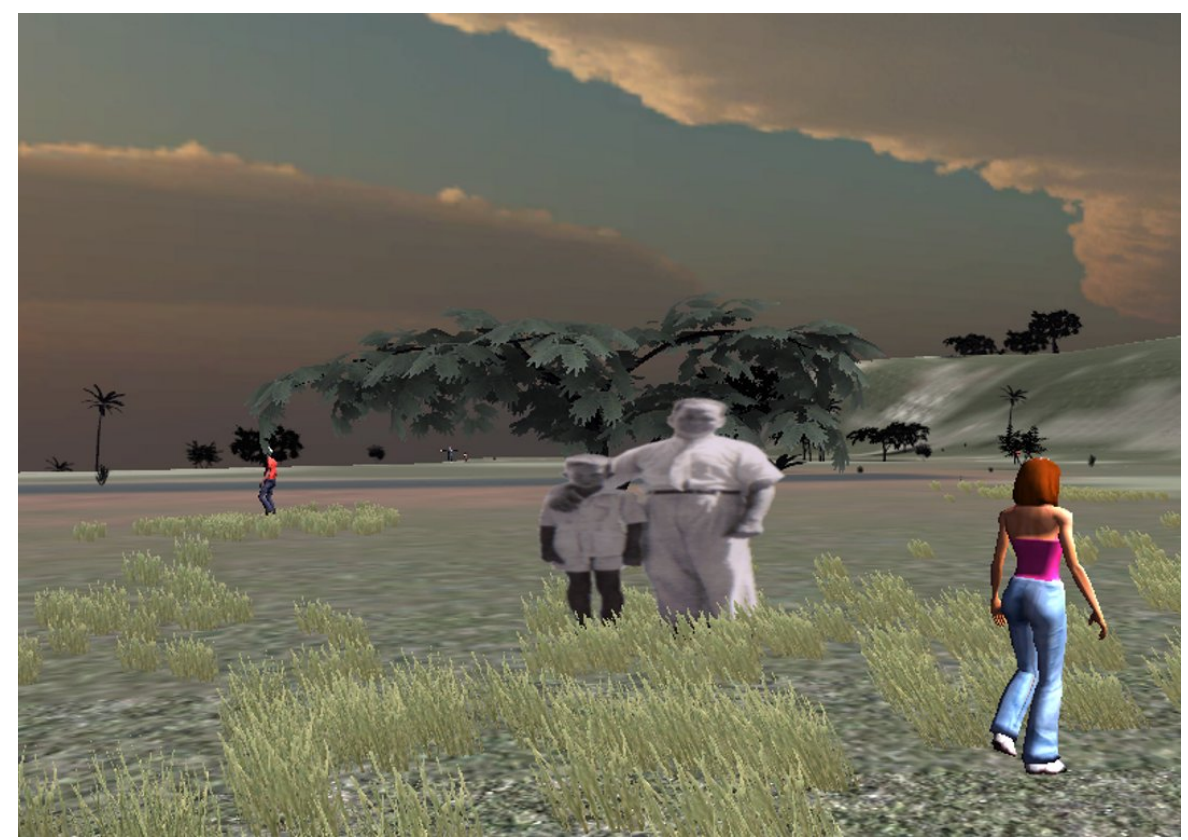

Figure 7.1: Different modes of representation coexist in WisLM. Here, a storyteller walks by a photograph of a pair of former 'Laurentinos' generously shared by the participants.

\subsection{Context: Mozambique 1974-76 ${ }^{1}$}

The independence of Mozambique was officially declared in 1975. This happened as a consequence of a turbulent period when diverse extraordinary vectors converged to make history. In the early 1960s, the Portuguese colonial policy was criticized openly in the United Nations, and the newly elected president of the U.S., J. F. Kennedy, boycotted Portugal in what was supported by a choir of two dozen new countries, which had emerged from the collapse of the British and French empires. With the loss of the city of Goa to India, the Portuguese empire was reduced to the 'overseas provinces' in Africa, East-Timor and the city of Macau.

The first steps towards a definite turn on politics for the African territories began in Angola in 1961, with the massacre of the 15th of March. During the following years the resistance grew, developing into widespread guerrilla warfare with three warheads in Guinea, Angola and

\footnotetext{
${ }^{1}$ In this section, I want to describe the sequence of major events immediately preceding the escape. It is a biased version of the history because it is history perceived from the perspective of those who left. It helps us to understand the frame of mind and reasons why they did so. It denotes how history is written in secondhand and somehow illustrates one of the main topics that this work addresses: the subjectivity of memory.
} 
Mozambique. This war lasted for 14 years and its end signified the end of the Portuguese empire and it is illustrated by a famous air bridge which, in 1975 and during 140 days, evacuated half a million refugees from Angola. The revolution of April 1974 overthrew, the totalitarian regime of Estado Novo and established a democratic regime in Portugal. The revolution in the metropolis reverberated like a seismic wave on the life of the colonies ${ }^{2}$. Its main lead agent was an army tired and worn out from an everlasting war. The new transition government in Portugal, heavily pressed by the international community, hurriedly negotiated the autonomy of the territories with the pro-independence forces.

The events in Angola set the tone for Mozambique's negotiations. If in Angola the bloodshed was dramatic, the war in Mozambique took place mostly in the North and far from Lourenço Marques. But in Lusaka, during the peace talks, the 'terrorists' (or 'turras', the nickname given to the pro-independence forces by the colonial forces), shown the recently conquered barracks of Omar and Nangade to be a major trump in their strategy of all or nothing for independence. On the 25th June 1975, and without any transitional period, Mozambique became an independent country. The exodus from Mozambique did not reach the epic contours and proportions found in Angola. Prior to the independence, and after the revolution in Portugal, there were some clear warning signs, in particular the failed attempt of white secession on the 7th September 1974 and the fights that followed on the 10th of October in Lourenço Marques. The escalation of the confrontation, and the proximity of the events, with some unfolding in the heart of the capital, had as an immediate consequence the escape of thousands to the neighboring countries of South Africa and Rhodesia (nowadays called Zimbabwe). After the independence, the social tension and the orthodoxy of the new communist regime from Samora Machel convinced many others to withdraw from the territory and find refuge elsewhere. Many of these families were breaking with a history of generations. In the article Adeus África, volto já, we can read that the sudden arrival of the colonial refugees to Portugal, with their suitcases and their boxes laying on the docks of the former metropolis, had the effect of a social earthquake. They became powerful agents of modernization for mentalities, but they were also blamed for the collapse of the empire [168]. In recent years (mostly after 2000), a boom in literature about the return was witnessed, made by and for 'retornados'. 'Retornado' is popular jargon to designate a person who came from Africa after the independence of the colonies, meaning literally: the one who returned. Quoting the journalist Raquel Ribeiro 'the history didn't lie saying that many came with only the clothes they had on their bodies to a country where they had never been before and where they were

\footnotetext{
${ }^{2}$ Large territories, such as Angola and Mozambique, were named successively by colonies (until 1951) as overseas provinces (1951-72) and finally as states (1972-75).
} 
christened "retornados", [208]. She continues "it was necessary to wait more than 30 years... to write about the stigma of being 'retornado', fundamental to understanding what it means to be Portuguese, today.'

It is in this context that this work emerges. It aims to give a voice to those who returned and the refugees; those who remember a 'past with no future'; those who saw 'dying their splendor in the grass'; those who have 'lost the possibility of another future' ${ }^{3}$. 'The literature of return is a special form of mourning... the mourning that many Portuguese didn't do', writes Eduardo Pitta in Memorabilia Ultramarina [191]. Extending Pitta's sentence by replacing 'literature' with art, with this work I am interested in the construction of a mosaic of voices, on building a platform for gathering accounts from the daily life in the city. How was Lourenço Marques?

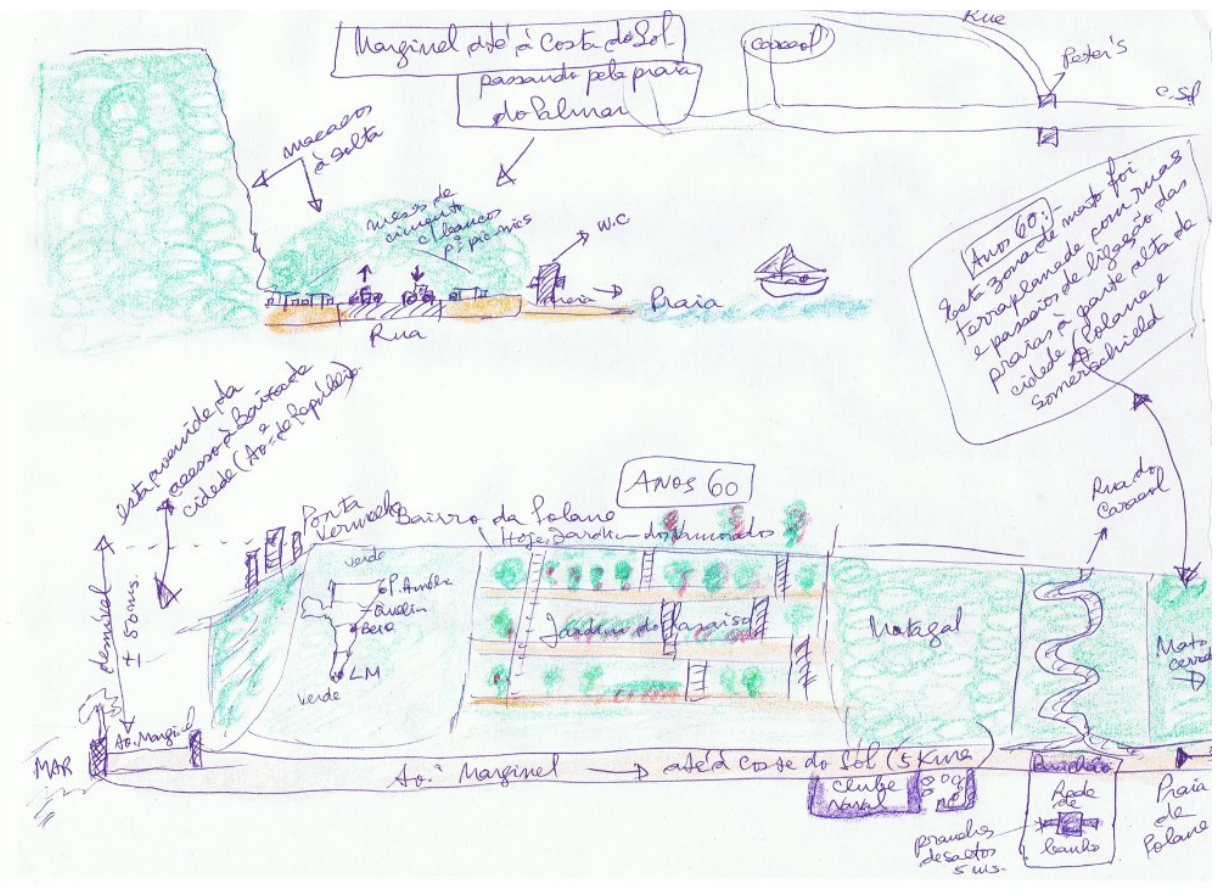

Figure 7.2: Diagram from a section of the city provided by one of the participants.

\subsection{Methodology: Chasing stories}

This work is a representation of the city 'as it was'. Because a city, more than its buildings, is the people who inhabit and experience it, this work departs from the accounts of those who inhabited that place, during the colonial period of Portuguese domination. Where is Lourenço Marques? (WisLM) is a collage of interrupted narratives, which takes expression

\footnotetext{
${ }^{3}$ These are quotes extracted from the interviews.
} 
in a virtual world. Its essence echoes Italo Calvino's work If in a winter night a traveller, juxtaposing multiple broken tales. The oral testimonies and the media materials provided by participants were the means to build this collective portrait. A group of ten participants were first interviewed ${ }^{4}$. This process was the catalyst for a wider exercise of collective memory gathering. Each participant was asked to share his/her memories by means of answering two questions: a) How was the geography of the city back then? b) What music emerges first when you think about the city?

During the interviewing process, some participants have shared their emotional testimonies. Some others, however, went even further, and in a auto catalytic process, unveiled the intimacy of their 'shoe boxes', feeding the project with personal photos, postcards, newspapers, etc. WisLM departs from the author's subjective interpretation of this shared documentation. On the level of material organization, this work is a database of memorabilia. However, in WisLM, a spectacle is offered (in Barthes' sense). First, a 3d landscape was modeled. This included sculpting the topography and appropriate vegetation, a layout interpreted from collected photos, drawings and maps. This process also comprised recreating some iconic buildings, again with a free architectural interpretation of the three-dimensionality and space occupancy. Other scenographic elements appear dispersed in the landscape: a) billboards displaying postcards with aerial views of the city during the 1960s and 1970s arise in the virtual world's supposed location that the images themselves represent; b) semi-translucent photos of people emerge here and there, ghostly images where the spatial context was removed, leaving only faces and bodies in suspended gestures, gazing at the audience from a distant time; and finally c) a soundscape completes the dramatization, with the music referenced by participants during the interviews, punctuating the space of the virtual world. Wis $L M$ remediates this database of memorabilia in a three-dimensional platform. An essential component of the work is the navigation throughout this landscape. The audience needs to traverse the space to map out the environment. Lev Manovitch equates these movements in the $3 \mathrm{~d}$ space with ancient forms of narration (fables, mythology) or 20th century American novels and romance (Mark Twain, Hemingway), where the action follows the spatial movements of the hero [150]. The narrative here is constituted by the sum of multiple trajectories through the database. As with many $3 \mathrm{~d}$ games where game-play draws on the user building a character by moving in the virtual space to collect new props and skills, moving in the space of WisLM, the audience navigates collecting elements of a shared memory. A sense of the past emerges from this spatialized collective composition.

\footnotetext{
${ }^{4}$ The interviews were in the Portuguese language, and later these were translated in order to be displayed as bilingual subtitles in WisLM.
} 


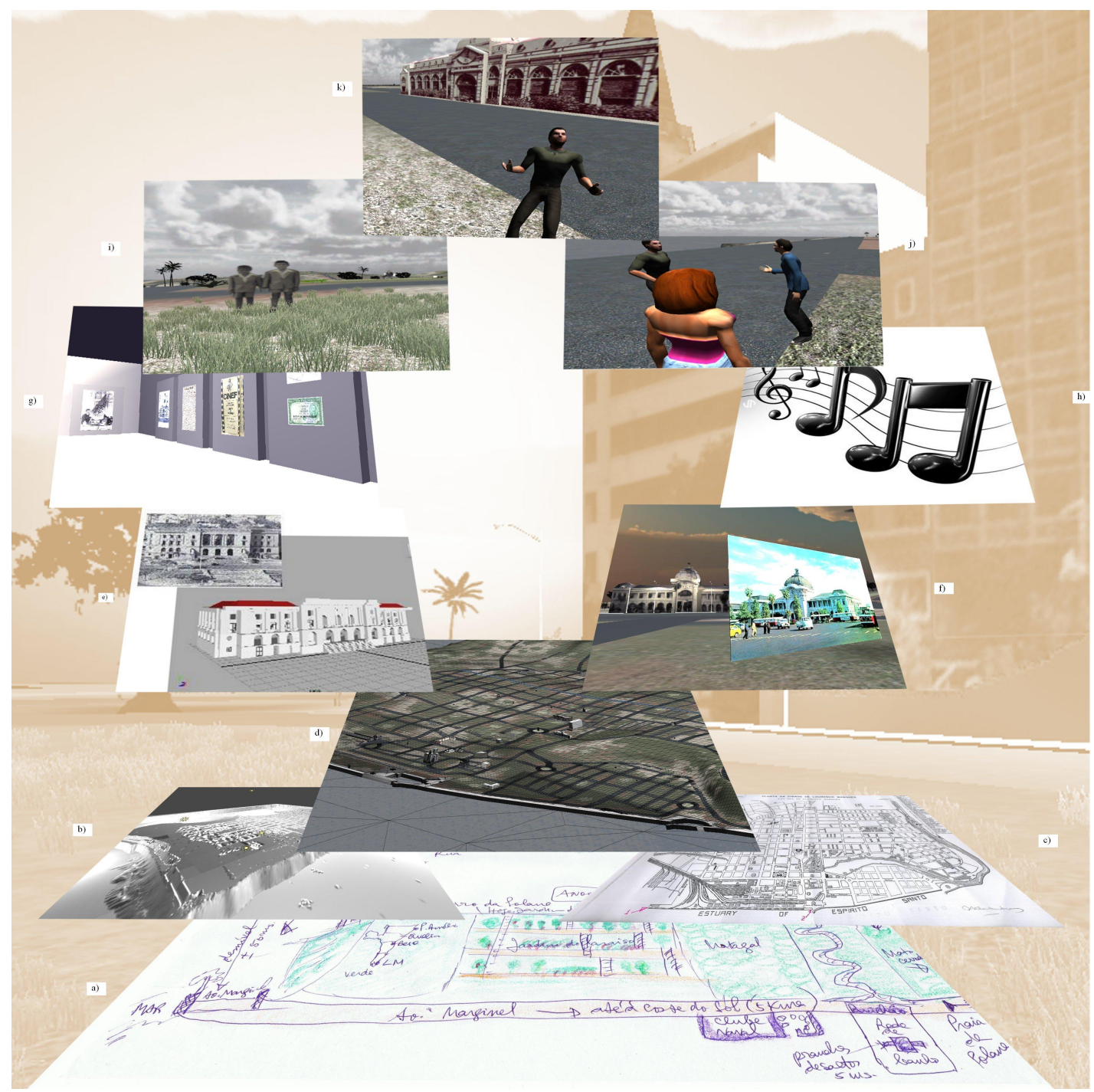

Figure 7.3: Schematic representation of the virtual world showing the different layers of representation. From the bottom to the top, and from left to right: a) Shared schematic drawings; b) Sculpted topography departing from the descriptions and materials; c) Shared maps; d) Road layouts after the originals extracted from the maps; e) Sculpted buildings from the interpretation of $2 \mathrm{~d}$-images; f) $3 \mathrm{~d}$ and photographic representations of the same site appear juxtaposed; g) Virtual museum with documents; h) Music originating from the question "what is the first song that arises when you think about the city?"; i) Images of 'retornados' removed from their background; j) autonomous population of NPCs; k) storyteller.

From interviews to avatar 'storytellers' However, the iconography of WisLM does not exhaust itself in the static elements strategically located and modeled, thus forming a spatial composition to resemble and represent the city. The second layer of this work draws 
on the oral accounts generously provided during the interviews. It translates experiences into memory objects. A population of animated storytellers inhabits the virtual city described so far. Helping to remember the lived experience, each of the individuals from this population of animated humanoid avatars is the carrier of one fragment from an interview. When navigating the cityscape, the audience selects (e.g with a mouse-click) any of these characters, who then stop their current activity, stare at the viewer, and gesticulates as if they were talking, while an audio recording is streamed and played. The audience is implicitly invited to seek-out these story tellers, 'hunting' them through the city, in order to listen to their stories.

The allegory of the computational ecosystem The model of Artificial Intelligence used to animate these characters is a Computational Ecosystem. As said, in earlier chapters, a Computational Ecosystem is formed by a community of autonomous agents, organized in a food web. Each of the agents in the community emulates a simplified life cycle from carbonbased life forms. The community evolves in a Mendelian-like process. Genetic traits, such as size or speed, are passed from parents to children when individuals replicate in a process emulating sexual reproduction. Energy is required for the individual activities, such as moving, running or simply breathing. The population competes for energy and space, and this trade unfolds within predatory acts. When the energetic level of one individual is too low, it is considered to be dead and as a consequence it is removed from the community.

An underlying layer of narrative is inscribed in the behaviours of this population. In the literary tradition of allegory (George Orwell's Animal Farm for instance), the social interactions performed by the storytellers in the virtual world are dictated by their performance in the habitat. Instead of primal animations of animals attacking and devouring each other, what is offered to the audience in WisLM are animations of gesticulating humanoids interacting in apparent conversation and discussion. Each action in the ecosystem is translated onto an animation of a corresponding gesture. For instance, when two individuals interact, if one is attacking the other, the gesticulation appears to be wider than the movements displayed by the same two individuals when these are playing.

The two-layered organization of the ecosystem, with producers and consumers, is explored in this work as to take advantage of the existing predator-prey dynamics, and in order to build up a narrative depicting colonials and servants. Individuals are identified by a Genomelike string, where each bit or combination of bits corresponds to certain phenotypic features (this implementation is explained in detail in the next chapter). Individuals are situated in the organization of the system - the food chain -, depending on the translation of their genes. When the genome has the 'servent' gene the individual in question becomes a producer. All the 
others are consumers. When consumers are hungry they search and hunt prey. The sequence of bits in the genome establishes the metabolic pattern that defines the position of one individual in the hierarchy of consumers. One sequence defines the chemical composition and a second sequence defines the metabolic possibilities for this individual. As such, consumers may prey on producers as well as be cannibals and live on the energy from other consumers at lower hierarchical levels. On the contrary, when producers are 'hungry' they move to farms where they will work until their energy and chemical balance gets replenished.

One interesting property about computational ecosystems is their capacity for the emergence of complex social interactions from the simple rules determining the behaviour of each agent. Pierre Lévy equates the virtual with potential [142]. Departing from one present situation - the actual - , the virtual is composed of all multiple possibilities that this specific context permits. In WisLM, different forms of social interaction are implicit in the initial design of the agents' rules. The virtual as potential are the different forms of interaction emerging from the design. Will the evolution of the virtual city unfold tragically as the original one? The social context of European colonialism, and in particular of the city of Lourenço Marques, can be metaphorically described as of predation from some social groups over the others. In the virtual city, in the long run, will we see interactions where mutualism and commensalism overcome predatory acts?

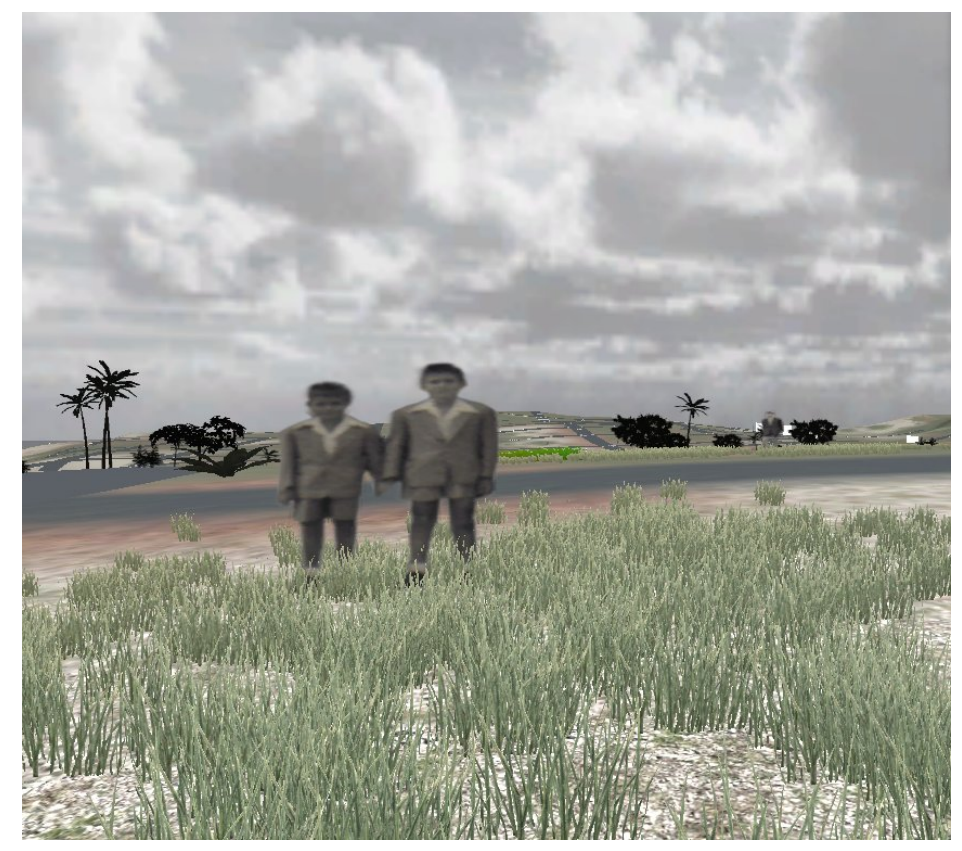

Figure 7.4: Two kids staring at the audience from a distant past. The original black and white photography is from a shared personal photo-album. 


\subsection{Discussion}

\section{Mediated Memories in a Virtual World}

WisLM offers a platform consisting of individual memory dialogues with extended contexts of community and history, capturing multiple dimensions of human life and experience. As with memory, there is a joint interpretation of objective facts with imaginary elements. WisLM questions the recall of the story, the metamorphosis that occurs when memories are converted to speech; it is in essence a work addressing the subjectivity of memory and the past.

This is a line of work inspired by previous developments of the use of technology for the preservation of culture, of historical heritage or social collective, such as Rome Reborn [106], Pompeii [149], the Forbidden City [125] and Uruk [45]. There is a lineage of works focusing on the reconstruction of sites historically relevant for human heritage. Rome Reborn, for instance, is a historically accurate reconstruction of part of the former imperial Rome in $3 \mathrm{~d}$ where visitors can interact with buildings and solid objects. The recreation of Pompeii also offers a large virtual crowd composed of humanoids dressed as Pompeii citizens walking around the reconstructed city. These are just for decorative purposes and are not interactive. On the contrary, in the reconstruction of Beijing's Forbidden City, the palace and home of China's emperors for 500 years, a small number of avatars interact with the users, thus guiding their visit. The scripted behaviours of the tourist guides in the Forbidden City are relatively simple when compared with the behaviours of the population in the recreation of the ancient city of Uruk, one of the first agglomerates of humans. Here, a population of NPC interacts, chatting intelligently with the audience.

This is a genre of work that partially informs WisLM. A second line of inspiration is provided by works, such as the Shoah Video Foundation [96], The Memory of the Netherlands [1] or Gone Gitmo [72]. These are works attempting to preserve culture in its sense of living experiences of people, etymologically searching the 'place' rather than the site. In the Shoah Video Foundation website, we can read the organizer's intention of using technology to 'preserve to perpetuity' the memories of the Holocaust survivors [96]. The Shoah foundation was founded by Steven Spielberg with the intent to 'gather video testimonies from survivors and other witnesses of the Holocaust'. The archive has nearly 52,000 video testimonies and is now hosted by the University of Southern California.

Another ambitious project that aims to collect and digitize hundreds of thousands of media materials relating to the past is the The Memory of the Netherlands. This project is described as a media gallery aiming to make accessible, through the Internet, collections of museums, archives, and libraries about life in the Netherlands' past [1]. 
The mediation of technology plays also a central role in Gone Gitmo [72]. This project is a recreation in Second Life of Camp X-Ray in Guantanamo Bay, the temporary detention facility where terror suspects were held and reportedly tortured. Gone Gitmo invites visitors to experience being a detainee for themselves. Besides the recreation of the physical space, accounts from former detainees are available as multimedia assets in the scenario of the virtual landscape. The authors have coined the term 'immersive journalism' to describe this form of documentary in a $3 \mathrm{~d}$ space. In all these works, the latest technologies play a central role in the process of evoking and remembering the past. Technology has always played a determinant role in this matter. Human memory is fallible; with time, the precision of some human memories blur as some become more and more diffuse. Others on the contrary become more vivid. From accounts written in papyrus, shoe boxes with old letters and photos, or even in the latest photo updates on Facebook, technology appears to be omnipresent and plays an essential role in the way we remember the past.

In the third chapter of Matter and Memory, Bergson challenges the idea of pure memory preceding its materialization in a mental image. The author theorizes memory as a subjective construction with memories being formed in the instant of its recall. In his words: 'to picture is not to remember.' Memory appears to be an assembly of facts, fantasies and fictions, subject to revision and change, and permeable to projections and desires [38]. Technology appears, in this context, to counteract and minimize erasure. Cultural theorists and social scientists talk about 'mediated memory' to refer to the incorporation of media in the process of remembering. Mediated memories do not simply invoke, but help to construct a sense of the past. WisLM is a panorama of lived experience, a representation which takes expression in a virtual world, an artefact built to converge some of the main technologies of our age: the database, the navigation in three-dimensional spaces, and the internet. WisLM is a complex exercise of mediated memory: it builds an emotional bridge between the past and the future.

\section{The representation of a trauma}

Despite the combination of technologies of the memory that are put together in WisLM, this is not described as an archive, but rather as an exercise of representation: the author's representation of the geography of the city. The behavior of the 'storytellers', the AI implemented in the characters, opens a discoursive layer that changes the ontology of this work. As discussed in chapter 5, a story may be modeled on the structure and organization of the algorithm. A connotative level is associated with the rules implemented, and each rule builds up and contributes to the sub-text of the work. WisLM is an allegory for a drama of recent human history. Individuals live off of the energy they capture from other members of the community. This 


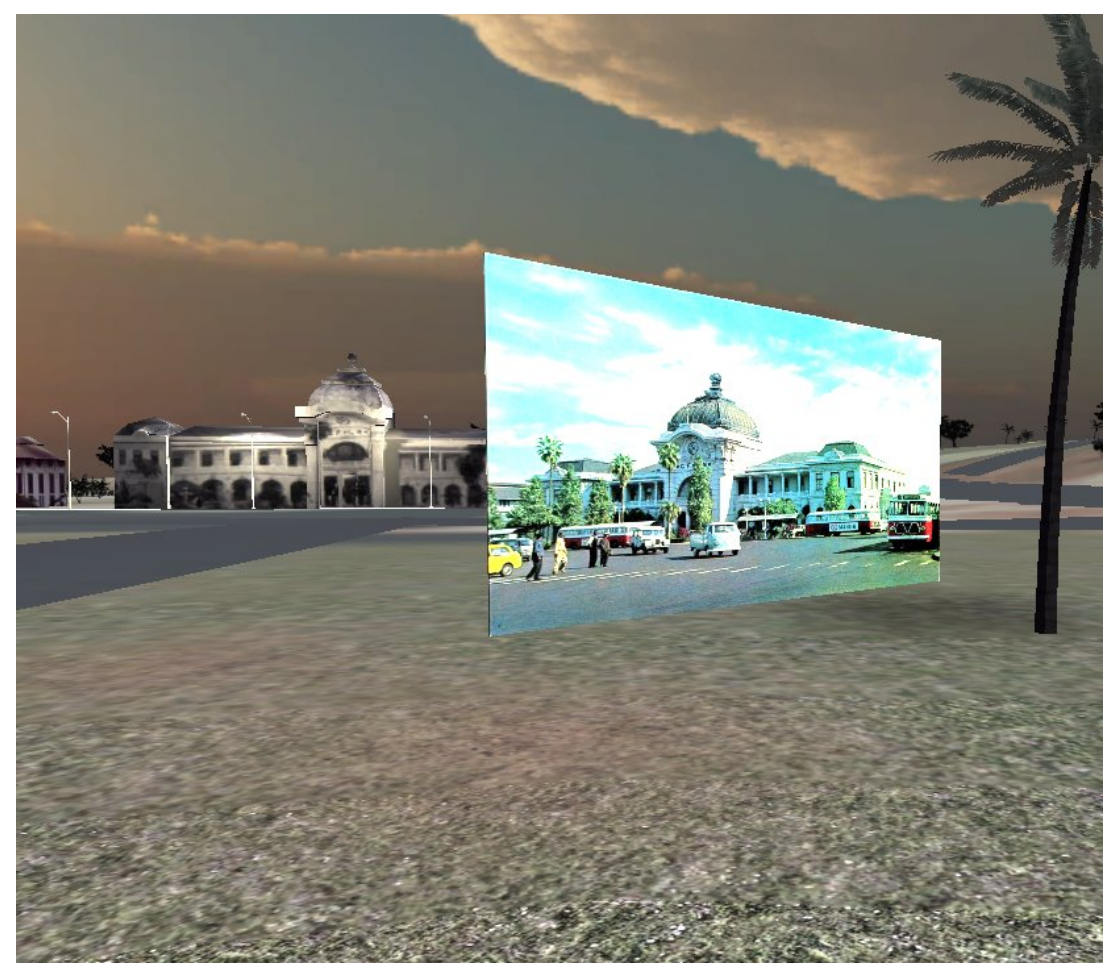

Figure 7.5: WisLM draws on the tension of juxtaposing multiple modes of representation. Here the $2 \mathrm{D}$ and the $3 \mathrm{D}$ versions of the train station appear in the same point of view.

type of interaction may be described as forming the basis for an economical metaphor since there is a currency being traded in the form of energy and 'chemical' components. To illustrate European colonialism, the hierarchical organization of this community is determined based upon genetic features. In the second version of the work, there is even a clear separation between those individuals who work on the farms to generate energy at lower trophic levels of the food chain (the producers) and those that are organized at higher levels (the consumers). Consequently, the latter are structured around the work produced on these lower levels. As these features and organization are genetically determined, this provides a paralell with the recent drama of colonial Africa.

Yet the behaviours rendered visible when we access the world are not immediatelly readable by the regular member of the audience as the underlying actions that have triggered these movements in the ecosystem. The food chain hierarchy is not visible, and the same goes for the predatorial acts. What we see instead are primarly individuals gathering in groups and talking, gesticulating. Some of these may even work on farms. Nothing seems to indicate a complex social structure. The conversational gesture associated with 'eat' that is rendered visible has no obvious and visible relationship with the action of 'eating' performed in the ecosystem. There 
is a fundamental piece missing in the linguistic jigsaw of meaning, discussed in chapter 5 . There is no explanation being offered to the audience accessing the work (either in the gallery or on the internet) of the underlying mechanism implementing the AI - this information is only available in academic papers. This more or less obscure layer is justified by the Lacanian tripartite scheme of the human mind: imaginary-symbolic-real [136]. The symbolic order uses metaphors and other signs to participate in the social sphere and communicate. However when it tries to communicate it leaves the real behind. The real is lost in the process of communication, it is something we cannot perceive. It is what is left after signification and meaning, after the symbolic order. It is the realm of trauma and the fixations that do not disappear. We try to articulate and represent it, but we fail. In WisLM, the real is built upon the algorithm, in the impulses and chains of actions and reactions forming the ecosystem where individuals are preying on each other. Like the trauma that the work addresses, the colonial drama the characters represent, the real of WisLM is there, omnipresent, yet it is unreachable and invisible, unatainable.

\section{Narcissus and the lake}

Another line of discussion that this work generates is about the interaction of Evolutionary art with contemporary art aesthetics. EvoArt has reached a stationary stage of evolution in its development. In the early 1990s, the work on EvoArt generated great excitement, and some significant methodologies and milestones were established. Today, these landmarks appear to be extremely difficult to surpass by the community of practitioners. In general, the work on EvoArt swirls around Karl Sims', William Latham's and Tom Ray's foundational ideas. It seems as if EvoArt has reached an evolutionary stable plateau, or adaptive peak, from which it cannot move far enough away to renew itself. What emerges from epistemological accounts of virtual ecosystems $([7,47,80])$ is what seems to be a recurrent pattern drawing on methodologies with resonances from the exploratory power of genetic algorithms, as the means to research aesthetics spaces. The individual agency from the virtual population generates and promotes a dynamic of novelty and surprise in the works; this dynamic requires a long-term incremental evolution with continuing emergence of the autonomous populations (discussed in chapter 2). Works revolve around the paradigms presented by pioneers such as Sims and Latham, and on the other hand Yager and Ray. As McLuhan reminds us in his discussion on media, the etymology of the word 'narcotic' comes from the myth of Narcissus. In the classic myth, Narcissus fell in love with his own image when this appeared reflected on the still waters of a lake, making him so obsessed and in love with his own reflected image that he forgot the world around himself. Evolutionary art seems to be somehow narcotized with its obsession 
with its own methodologies and processes (something already pointed out by McCormack in [157]).

The remediation of CEs in VWs creates a situation of mutual advantage, offering a chance to renew the repertoire of both VWs and EvoArt, both artistically and technically. The participative paradigms that characterize Web 2.0, and currently inform VWs, may be key contributors to renew EvoArt with new dialectic territories, shifting these from interactive paradigms to participative ones.

Critics such as Nicolas Borriaud and Claire Bishop play a fundamental role when looking to much of the contemporary visual arts practices of the last twenty years. They advocate that what renders it distinct and unifies the most recent contemporary art are people, their lives, and their relationships in context, 'the realm of human interactions and its social context' [50]. Human stories and experiences of life are indeed at the core of artistic discourses and practice of artists as diverse as Felix Gonzalez-Torres, Liam Gillick or Tracy Emin. In Relational Art, the audience is envisaged as a community, and interactivity and inter-subjectivity appear at the core of the practice. In Borriaud's words: 'they are at once the starting point and the point of arrival'. The artists work within the sphere of inter-human relationships and 'the artwork creates a social environment in which people come together to participate in a shared activity'. He continues, 'Rather than the artwork being an encounter between a viewer and an object, relational art produces inter subjective encounters. Through these encounters, meaning is elaborated collectively, rather than in the space of individual consumption' [50]. Evo-artists seem to be hinting at these words, and works such as Eden or Artificial Natures appear to put interactivity at the top of their priorities. The audience plays a role in the interpretative process while engaging in a ludic experience.

The movement in WisLM is the shift from interaction towards participation. Earlier, Technosphere has shown that the ontology could be shifted slightly. Community is generated when internet forums related with the work are created, when users comment and show each other their creatures. Users are indeed participants as they are the ones who create the creatures [192]. This is something explored in the game, Spore [26]. With WisLM, people appear similarly in the beginning and in the end of the process. Interviews forge encounters, community is generated in the process. As Bishop suggests, the artwork is just a channel for a wider participation. The final exhibition of WisLM is planned to bring the work to a community where wider participation is generated by means of a dancing-tea event ${ }^{5}$. Evolutionary art is simply the channel. Moreover, the experience with $x T N Z$, Senhora da Graça and WisLM brings to

\footnotetext{
${ }^{5}$ This is reminiscent of the popular dancing afternoons organized by the main radio station in Lourenço Marques.
} 
the core of the practice the subjective experiences of life from the author. This is something barely existent with previous practitioners, who in general detach their life experience from their practice. Rather than advocating radical gestures and manifestos in the spirit of the avantguards, my proposition is that contemporary art theory already provides the tools and means to inform generative and evolutionary art with novel perspectives. This argument should not be conceived as passing an aesthetic judgment on the relative validity of works under consideration. However, it is clear that the work presented in this chapter poses a completely new problem: it builds on "the symbolic value of the "world" it suggests to us and the image of human relations reflected' [50]. The dialetics of EvoArt artworks appears enriched with a social dimension. WisLM was created by collective participation, bringing together a community. It addresses people and their stories. It also challenges users to become participants sharing their documents and stories, helping the system to grow. This is the direction driving and inspiring the construction of this work, to bring together people, their stories, experiences and participation and articulating these with an evolutionary population and paradigm. 


\section{Where is Lourenço Marques? - II}

\section{An Ecosystem Based Model for Real-time Generative Animation of Gregarious Humanoid Non-player characters}

\subsection{Introduction}

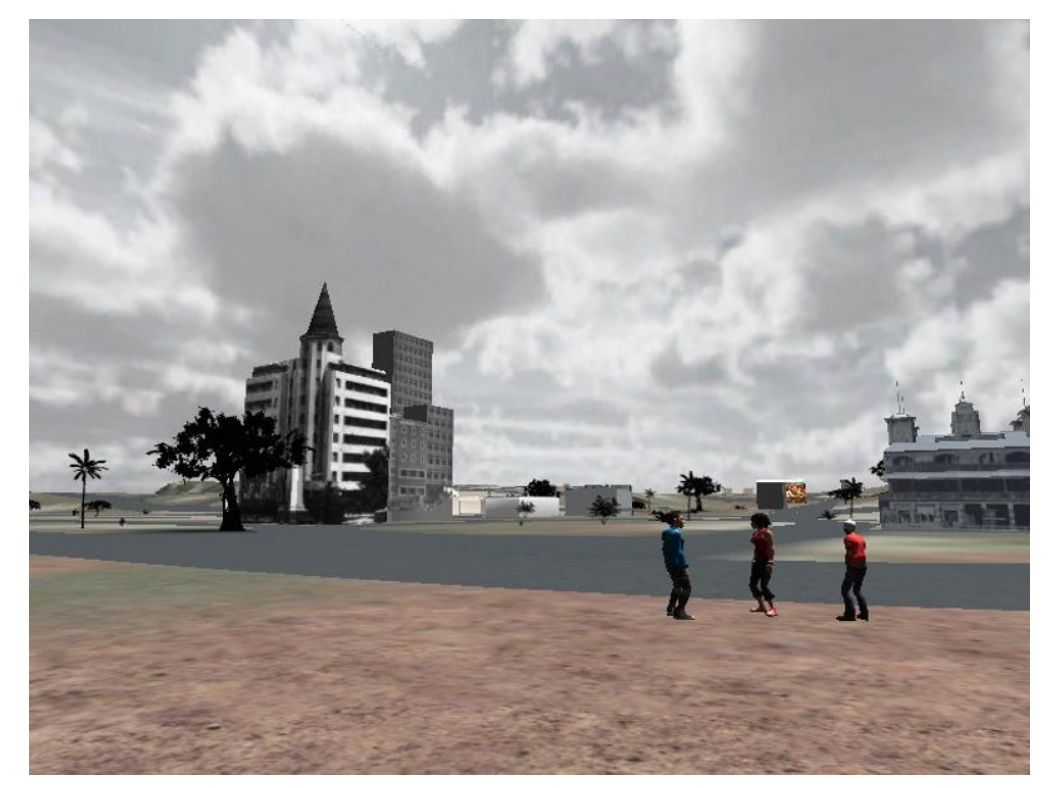

Figure 8.1: Overview of the virtual world. In the background are some buildings and billboards, and closer, some members of the community of storytellers in interaction.

Animations of crowds in the historical site of Petra, Jordan, the Pennsylvania train station of New York City [218], or of visitors in theme parks [123] are good examples of a developing area of research that looks at modeling virtual spaces inhabited by communities of humanoid avatars that self-organize and interact autonomously. Traditionally, the algorithms that model groups of individuals inform one of following main approaches: when these individuals a) 
are represented as particles subject to physical forces [110]; b) are represented as states of cells in cellular automata [32]; c) follow a rule-based scheme [206]. These approaches have the merit of modeling in a realistic way the macro level of the crowd and the features of the spatial flow. Recently, much attention has been put into the micro-level, which is centered on an individual's behaviour within a multitude. Some works attempt to recreate the spontaneity of behaviours visible in small groups of a few dozen individuals. Pelechano et al., for instance, model a group of humanoids interacting socially at a cocktail party [186]. Shao and Terzopoulus animate pedestrians in a station and theater goers in an ancient reconstruction with individuals exhibiting spontaneous behaviour, such as standing in queues or watching shop windows [218]. A good survey of the field of crowd animation up to 2008 can be found in the books of Pelechano et al. [187] and Thalmann et al [234].

The focus of this chapter is on the community of humanoid avatars which move autonomously around the virtual city of WisLM and interact with each other as well as with the human audience. These avatars are the agent 'storytellers' (Figures 8.2 and 8.12). When the user selects (points or clicks on) any one of these avatars, it interrupts its current activity and faces the camera. Then, animated and gesticulating as if it was speaking, such an agent streams an audio testimony of one of the oral accounts recorded during the interview process (Figure 8.12). Thus, this population of avatars assists in the task of bringing the experiences of the respondents to the forefront.

The aim is producing the visual effects of a community of gregarious humanoids with small interacting and interactive crowds (groups of 2 to 10 individuals, Figure 7.2). Motivated by the design specifications described in the previous chapters, which require the animations of a humanoid population driven by predatory behaviours, attention is paid in particular to systems where agents are organized in the hierarchical structure of a food chain while trading token units of energy and biomass as a way of promoting community dynamics. As discussed earlier, a $\mathrm{CE}$ is a generative model that provides for complex environment simulations rich in the targeted heterogeneity and spontaneity. 


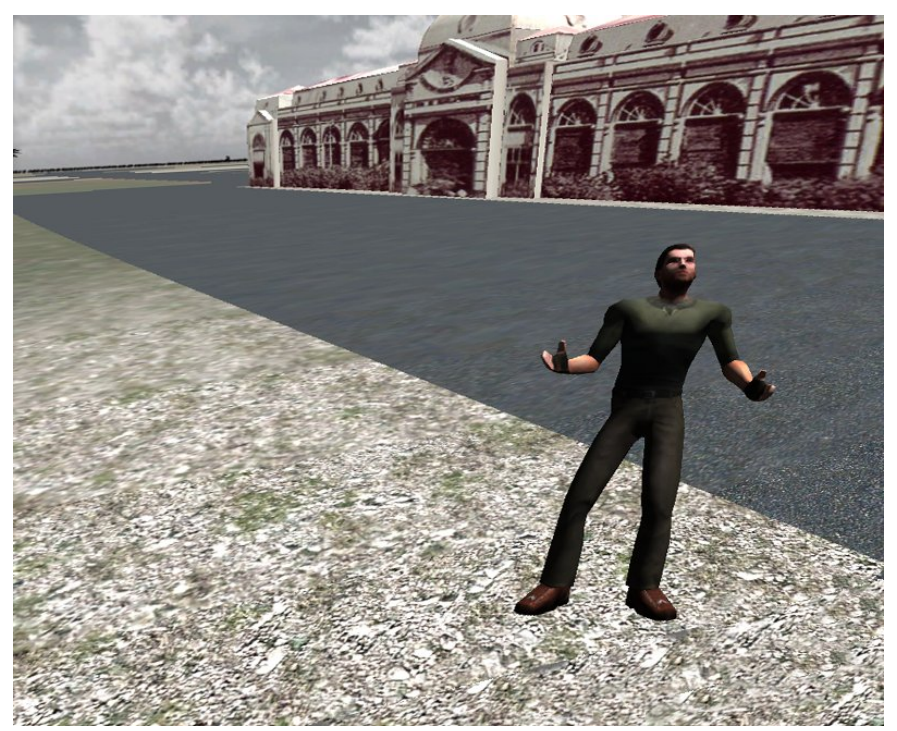

Figure 8.2: Image of a 'storyteller' in interaction with the audience.

\subsubsection{The computational ecosystem as an Al engine}

CEs have been used as generative engines in diverse contexts, such as audio-visual installations [83], music composition [90] or choreography of avatars ${ }^{1}$ in virtual worlds [23]. One of the main contributions to knowledge produced by this thesis is that this approach innovates by the use of the complexity and indeterminacy of a CE as an AI to coordinate the social movements in a population of humanoid NPCs (avatars).

By design, the observable behaviours of the population correspond to a visual representation of the ecosystem's inner-workings. The spatial layout of the virtual city provides support for the visualization of the underlying multi-agent state space, with a direct connection between the current state of the ecosystem and the configuration of the avatars in the layout. The birth of a character is represented by a new avatar appearing next to its parents, while its death is represented by its 'dematerialization' into thin air. Each interaction during the lifetime of an avatar is translated into a series of movements or gesticulations while being constrained to the surface of the world. The CE establishes correspondences between states, movements and actions performed by each avatar. For example, the action of an avatar feeding in the virtual ecosystem might correspond to a certain gesture in the animation, while its escape after a fight with another creature will correspond to a different form of gesticulation. In WisLM, a set of

\footnotetext{
${ }^{1}$ Each avatar is an individual belonging to the population, and I will refer to each one as an 'avatar'. The term avatar is often used in the computing domain as a graphical representation that mediates the user in a virtual world or game. The term is used here more generally since the character is equally animated by an external force: the algorithm which drives the behaviours of the character.
} 
nine base animations was defined. This small set is deemed sufficient for a prototype to allow to explore and demonstrate the potential of CEs in animating virtual populations of humanoid characters. The set includes the animation of walking and eight different arm movements (or gesticulations) used during conversations. In the elaboration of the behaviours of the avatars, a classifier system was implemented, which was inspired by the work Senhora da Graça [23] and is described next. The $3 \mathrm{~d}$ environment was implemented using the game platform Unity $3 \mathrm{~d}$ [241].

\subsection{Description of the system}

\subsubsection{The agent representation}

In this work, a number of techniques with a proven record of successful results in animating populations of multiple individuals are combined. These are: (i) a hormonal system as proposed by Daniel Jones in his framework for bio-inspired swarms [129], (ii) a metabolic system to define and restrict the diet of the characters based on the model of Saruwatari et al. [213], and (iii) a classifier system adapted from John Holland's model [119], which drives the action-selection mechanism of our characters. In the continuation, the implementation of each of these main techniques is described.

\section{The hormonal system}

Jones' framework for sound-based performance using swarm dynamics introduces a biomimetic design process that augments the classical rule-set for flocking, in part through the implementation of a hormone-like system which allows temporal modifications of the individual behaviour of particles within a swarm [129]. This architectural design was adapted to expand the classical energy paradigm used in more traditional CEs, which typically operates as a thermostat-like regulator [158]. Adding an extra layer of hormonal regulators increases both the life-likeness of this model and its associated complexity. This layer is defined via five variables: a) Testosterone - increases with age and crowdedness, decreases upon giving birth, and causes an increase in the likelihood of reproduction; b) Adrenaline - increases with overcrowding, decreases as a result of internal regulation overtime and causes a greater rate and variance of movement; c) Serotonin - increases with 'day' cycles, decreases during 'night' cycles, decreases as result of hunger, and causes a greater social attraction towards other agents; d) Melatonin - increases during 'night' cycles, decreases during 'day' cycles and also decreases the rate of movement e) Leptin - increases upon eating, decreases steadily at 
all other times, causes downward regulation of Serotonin when depleted, and causes greater attraction to food.

\begin{tabular}{|c|c|c|}
\hline Hormone & Description & Effects \\
\hline \hline ht & Testosterone & $\begin{array}{r}\text { Increases with age and crowdness; Decreases upon giving birth; } \\
\text { Causes an increase in the likelihood of reproduction. }\end{array}$ \\
\hline ha & Adrenaline & Increases with overcrowding; Decreases as a result of internal regulation overtime; \\
Causes a greater rate and variance of movement.
\end{tabular}

Table 8.1: The hormonal system, after Daniel Daniel [129].

\begin{tabular}{|c|c|c|c|}
\hline Gene & Description & Effects & Size (in bytes) \\
\hline gAge & Age & Rate at which the agent ages & 2 \\
\hline gPerc & Introspection & The level of gregariousness & 2 \\
\hline gCycx & Hormone cycles & The strength or speed of the hormone cycle \\
(e.g how much will increase per day) & 3 \\
\hline gUpx & Hormone uptakes & The strength or speed of the hormone cycle & 2 \\
\hline gChem & Body Chemistry & Chemical components present in the body & 3 \\
\hline gMetab & Metabolism & What chemicals can be 'digested' & 3 \\
\hline
\end{tabular}

Table 8.2: The Gtype, after Daniel Daniel [129].

\section{The blueprint descriptor}

These hormones are initially configured by the genetic descriptor (the Gtype) containing a blueprint for the physical attributes of the agent. This is a string with 15 binary digits, where different sections of the string code for specific features: a) Age (gAge) - rate at which the agent ages; b) Introspection (gIntr) - level of gregariousness; c) Hormone cycles (gCycl) - strength or speed of the hormone cycle (e.g how much will increase per day); d) Hormone uptakes (gUptk) - strength or speed of the hormone cycle (e.g how much will the hormone increase when colliding with others); e) Body Chemistry (gChem) - chemical components present in the body; f) Metabolism (gMetab) - what chemicals can be 'digested'. In the initial population, these features are determined randomly. Afterward, each subsequent generation inherits this 
information from their parents. This follows a Mendelian-like process of diploid reproduction, where crossover operators are used on Gtypes to promote variation. In the process, some noise is added to the inherited information to mimic mutations (with an arbitrary small probability $P m=0.05$ of effective mutation on each bit).

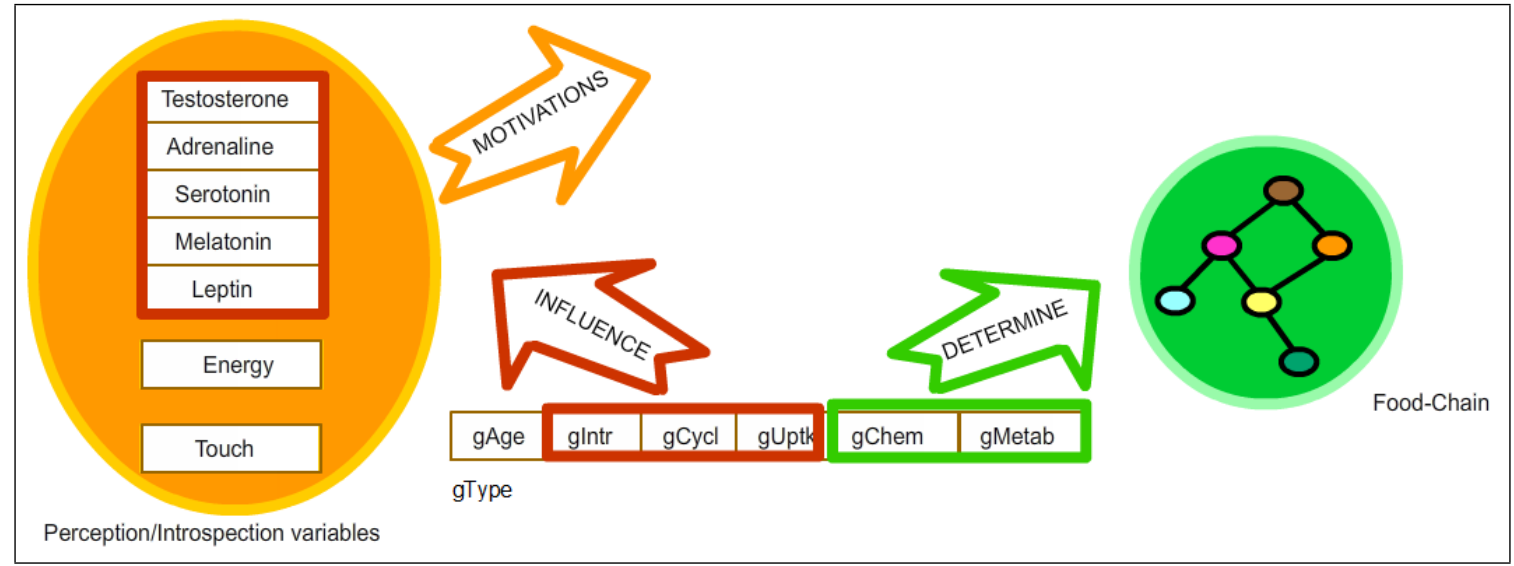

Figure 8.3: Graph depicting the GType influence on the behaviour of the character. The variables (gIntr, gCycl, gUptk) directly influence the hormonal system, which motivates the actions performed in the world; the variables (gChem, gMetab) set the body-composition and dietary specifications, which determine the environmental context for the actions.

\section{The metabolic system}

\section{The bodies chemistry and 'metabolism'}

Saruwatari et al. [213] provide a model that I found useful to determine the dietary specifications. Their framework uses two strings, the first of which defines the body constitution of the character, while the second is used to describe its metabolism. Potential prey are those whose constitution-string matches the predator's metabolic-string. Saruwatari et al. have shown this simple mechanism potentially leads to the emergence of complex multi-trophic food chains with variable depths, which in turn gives us the necessary differentiation and stratification required in this model.

The first part of the dietary specification is given by the string of 3 binary digits present in the Gtype section gChem. This represents the avatar's body 'chemical' composition. The first digit codes for the possibility of presence of the chemical A, the second for chemical $\mathrm{B}$, and the third for chemical $\mathrm{C}$. Each avatar is equipped with chemical repositories. These repositories are direct translations from the composition-Gtype gChem . Take for instance an avatar with gChem of ' 101 '. The only chemicals present in the repository will be A and C. 
Another avatar with Gtype '010' will only have repository B active. When a hypothetical avatar $(\mathrm{X})$ preys on another avatar $(\mathrm{Y}), \mathrm{X}$ will extract from the chemical repositories of $\mathrm{Y}$ the existing content, thus filling in its own repositories. In the process, $90 \%$ of the value is wasted. Each repository only carries a maximum capacity, which is directly related with the avatar's body size. The body size is given by a direct translation of the binary value of gChem. These chemical attributes play an essential role in the ecosystem since these determine part of the dietary specification in the community and thus the interactions of the individuals. The second part of the dietary specification is given by the gMetab component. This defines the avatar's metabolism, what chemicals this individual can 'digest'. For instance, an individual with gMetab 100 will be able to prey on individuals whose gChem codes the A component: 100, 110, 111, 101. Consequently, the combination gChem - gMetab establishes the fundamental interactions in the predator-prey relationships. This predator-prey mechanism of matching the metabolic-string with the composition-string provides an interaction space size of $64(8 \times 8)$, which was wide enough for this current work.

\section{The metabolic rules}

The metabolic system emulates a simplified food-energy conversion. Besides the chemical repositories and the hormonal system, avatars have one main structuring variable contributing to their behaviour: energy. This is generated from the chemical repository of the avatar. To emulate a conversion from mass to energy, an arbitrary chemical reaction was defined. This requires three chemical units to produce one unit of energy (e.g $2 A+1 B=1$ energy). Energy can be spent when breathing or performing actions in the world such as moving, attacking, running away, preying, eating or mating. Below a certain energetic level an avatar needs to generate more from its chemical-repositories. Below a certain threshold the avatar starts to feel 'hungry', activating the internal sensor. When the energy level reaches the value 0 , the avatar dies and is removed from the system.

\section{The behaviour system}

The characters' behaviour is defined by three main operational stages: perception, decision, and action. Each character is provided with: (i) internal sensors monitoring energy and hormonal levels, and (ii) sensors for contact, which are triggered by the proximity of other characters (Figures 8.3). As a function of these inputs, the character will choose the action to take using a classifier system inspired by the description provided by John Holland [119], a model that allows autonomous agents exhibiting self-organization capabilities and temporal 
adaptation. Holland's model was used in the well-known Echo system [97] and also inspired artworks such as Eden [158].

\section{The classifier system}

During the process of perception, the system inspects the level of energy and the state of the hormonal variables, as well as if the body of a character is entering into contact with any other characters. When any of these variables is activated, such as when: (i) the energy or leptin levels are below pre-selected thresholds, (ii) the testosterone, adrenalin or serotonin levels are above some pre-fixed thresholds, or (iii) the character is touching another body, then an action-message is generated. This message takes the form of a string of 6 in length, and is composed from the grammar set $\{0,1, \#\}$, identifying which sensor is active — binary values indicate the active and inactive states while \# functions as a wildcard.

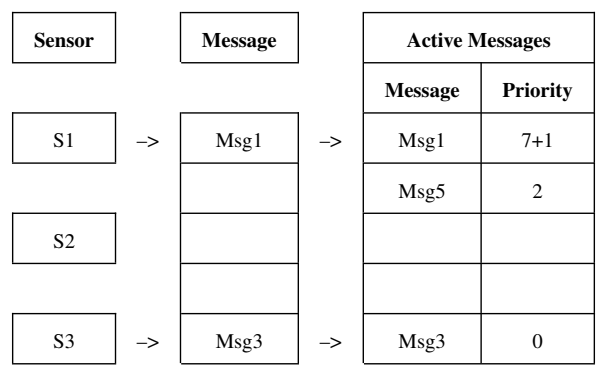

Figure 8.4: Illustration of a situation where the active messages Msg1 and Msg3 are generated due to the hypothetical simultaneous activation of sensors S1 and S3 in the perception state. The list of current active-messages is updated with the two new messages. Msg1 increases the priority of the existing entry for Msg1, whereas Msg3 updates the list with a new entry with priority 0 .

The active messages list This is a list with messages waiting to be processed. If the current message doesn't exist in this list, it will be inserted with an assigned priority of 0 . If, on the contrary, the message already exists, this means that the same sensor has already triggered one or more messages but these haven't yet been processed. If that is the situation, then the priority of the existing message will be increased by 1 . During the decision stage, the message with highest priority from this list will be removed and processed against the table of rules to generate actions.

The table of rules This describes a set of actions and their indexes. The rules are initially similar for all avatars. Each rule contains three parameters: index, action and priority. Similarly as before, the index is also composed from the grammar $\{0,1, \#\}$. This index is used to 
match the corresponding rules to the active message being processed.

Multiple rules can match one single message. Since the character \# functions as a wildcard, this means that any value can be accepted in the particular character of the index where the wild-card is located. However, as each of the rules has a specific priority assigned to it (the priority is initialized with a random value), of all the candidate rules (those which indexes match the message) only the one with the highest priority will be selected.

The action to perform is coded in the second section of the rule. This is an alpha-numeric code, which is translated into a procedural action, such as an instruction to prey on any avatar that is within a certain distance or an instruction to move towards the closest avatar, etc.

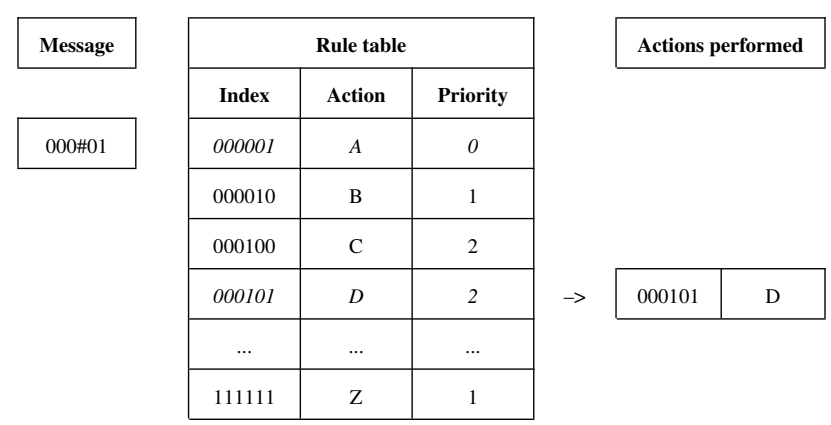

Figure 8.5: An illustration of the process of transformation from message to action in the decision state. The message 000\#01 finds two compatible indexes matching in the table of rules (000001 and 000101). From these, the action of the one with highest priority is performed.

The reward system The priority of the rules is updated according to the consequences of the actions performed in the world. An arbitrary reward was attributed to some of the possible actions, such as eating when hungry or victory/defeat in battle. If, for instance, the selected action is to feed, this implies a positive reward. On the contrary, being hit implies a negative value. The reward affects not only the direct rule, which has triggered the event as well as the rules for the previous actions. Each avatar has a FIFO memory, which stores the last 5 rules performed. This block of memory is also rewarded accordingly, with the rules being credited in a decremental way corresponding to the time they have been present on the memory. For instance, when an avatar manages to prey, the rule for the action which has triggered the preying event is rewarded with 5 . The immediate rule-action prior to that one is rewarded with 4; the anterior with 3, and so on. When a new event occurs and a new action is performed, the oldest rule-action is removed. 


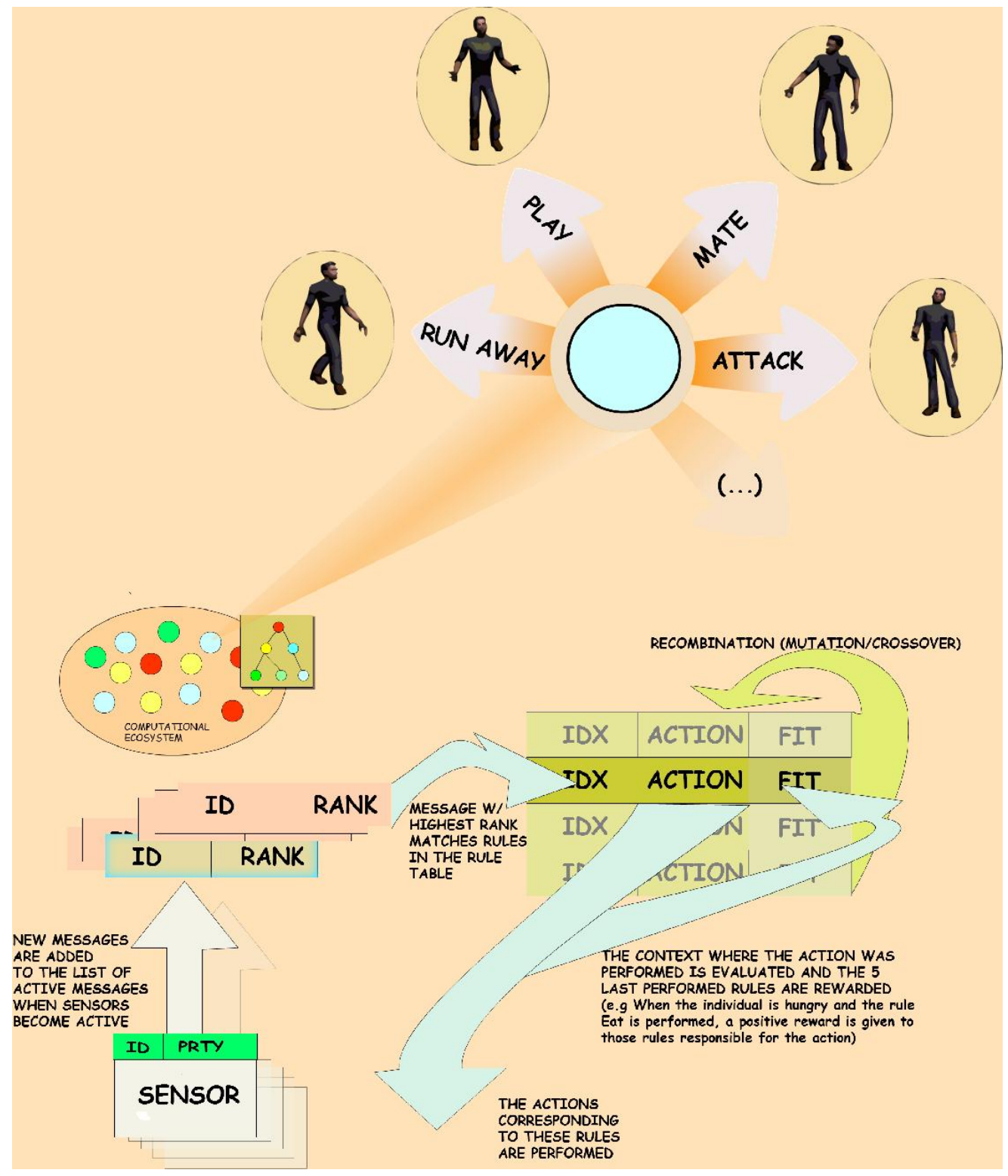

Figure 8.6: Graphic depicting the overall structure of the algorithm. At the bottom, the action selection mechanism is illustrated: sensors trigger messages indicating a specific need; these messages are prioritized and form a buffer, which is ordered by associated priority. The message with the highest priority is selected to trigger an action. At the top of the graph, the mechanism rendering actions is illustrated. For each syntactic action, an associated animation is played.

Generation of new rules During reproduction, the newborn also inherits from the current rule-table of the parents. To constitute the new rule-table, the rules with top priority from both progenitors are inherited in a 50/50 proportion. Each of these indexes in the new rules suffers a process of (possible) mutation. These indexes may suffer a transformation resulting from 
four possible attempts for digit mutation, each with a success probability of $50 \%$.

\section{The mapping of behaviours}

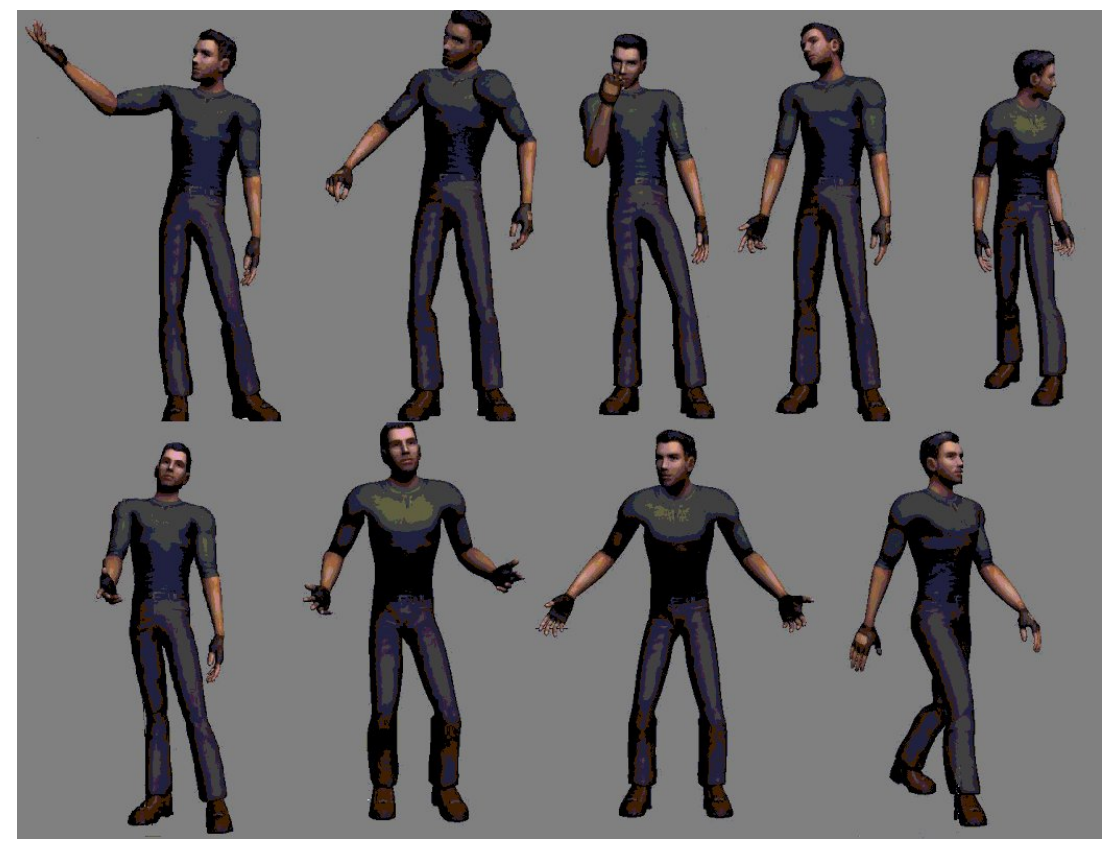

Figure 8.7: Still images of the animations corresponding to each of the actions performed by the agent. Above, from left to right: a) eat prey; b) attempt to mate with a partner that is not ready; c) reproduction or play alone; d) successful mating; e) attempt to mate but no compatible individuals exist in the vicinity. Below, from left to right: i) losing an attack; ii) play with happy mate; iii) victorious attack; iv) walking (move to mate, wander, move to prey);

As mentioned earlier, the actions are encoded in the second part of each rule. Each of these rules might trigger new messages or might generate some physical action from the agent, such as movement or talk. To render visible each physical action in the virtual world, one associated animation needs to be played. The relationship between the animations and the actions is rigidly defined a priori. For instance, for the rule triggering the action 'eat', it is the animation associated with 'eating' that will always be played (Fig . 8.7). These animations have been defined in a arbitrary way following the criteria of making sense in the context of the work. This type of contextual adaptation is similar, in a way, to Petty et al's use of a lookup operation in predefined tables to assign the actions to be performed to the current 'moods' of the agents [188].

The constant dynamics of the landscape is generated by the continuous movement of the characters in the space of the virtual world. These movements can be of two types: a) in 
the direction of an arbitrary 'preferential location', a random coordinate which, once reached, is reset with a new value; or b) in the direction of other characters as a consequence of the internal needs as determined by the scheme previously described. The former is the default mode when no stimuli is activating any actions, while in the latter the character will display conversational behaviours.

\section{Tuning the animation}

Two versions of the work were developed. The first was with a population composed of only consumers (homogeneous), which was the work exhibited at the Tin Shed gallery; the second version was developed to improve some problematic aspects noticed during the exhibition. This is the version where a population of producers and consumers was introduced as to better illustrate European colonialism. In order to improve the behaviours, some non-trivial measures were introduced in this second version of the work. Quite often, individuals happened to have conflicting motivations being processed almost simultaneously. For instance, their hormonal levels might be triggering messages to attack prey that is in the vicinity, but these would also tell him simultaneously to sexually approach a partner that is also in close proximity. As soon as one of the messages gets processed by the system, the other becomes a priority. When this situation happens they look to their targets in two opposite directions and interact repeatedly with these two separate individuals. In order to avoid that kind of situation, a rule was implemented to force the avatars to always look at the closest individual, regardless of which one they are interacting with.

The second non orthodox measure was to avoid repetitiveness during these interactions. At times, the avatars keep exhibiting the same gesture over and over as a result of their non satisfied needs. For instance if one is to attack another that is far weaker but resilient, the one attacking will be showing the animation 'attack and win' repetitively. In this second version, collisions also trigger animations in order to prevent this repetitiveness. For each collision happening to one avatar, there is a $50 \%$ probability that one of the eight animations will be played. Which of these it will be is decided at random. This adds some diversity to the movements and to some extent breaks with continued repetitive patterns of animation.

\section{The persistence of the system}

In chapter 6, four methods were discussed and compared to address the problem of the persistence of systems, such as the one described here. MIX was considered as one algorithm offering a solution that provides milder fluctuations of population density. This implements a 
technique that explores classical approaches combining them. Insertions are inhibited when the system is close to being overpopulated. Conversely, new individuals are added, replacing the ones that are removed by death when the population is next to a minimum-viable number.

\subsection{Results}

WisLM has shown some interesting results based on a limited set of predefined movements. The system was tested in a public display at the closing ceremony of the Watermans Festival of Digital Art 2012 and the Tin Shed space, both in London, with positive feedback from the audience. Twenty four responses were obtained from anonymous users. These users responded to a questionnaire where $80 \%$ said the group formation and the talking movements in the simulation appeared to obey an internal and coherent logic, whereas $65 \%$ said the behaviours were realistic in a cartoon way (Appendix-C).

The performance of the system was also monitored. WisLM was set running continuously for a period of 7 hours, a period of time deemed sufficient to emulate one day of exhibition. An image of the system's state was captured at every 15 seconds, a short interval (4 snap-shots per minute) which allowed the production of a detailed portrait of the behaviour of the system. Under scrutiny were a number of variables, such as the heterogeneity of a population generated, the spatial distribution, the heterogeneity of the actions performed, and the population density.

\section{Heterogeneity of population}

Fig 8.8 illustrates the distribution of the genotypes of the population. This is given by the overall quantity of Gtypes present in the population. Recall that there is a limited number of possibilities of Gtypes available, as the length of the genome is of size 15, a space of 32768 possibilities. The abscissa represent the Gtypes. Results are presented in alphabetical order, thus relatively closer Gtypes in term of their binary distance might appear largely separated on the graph. 


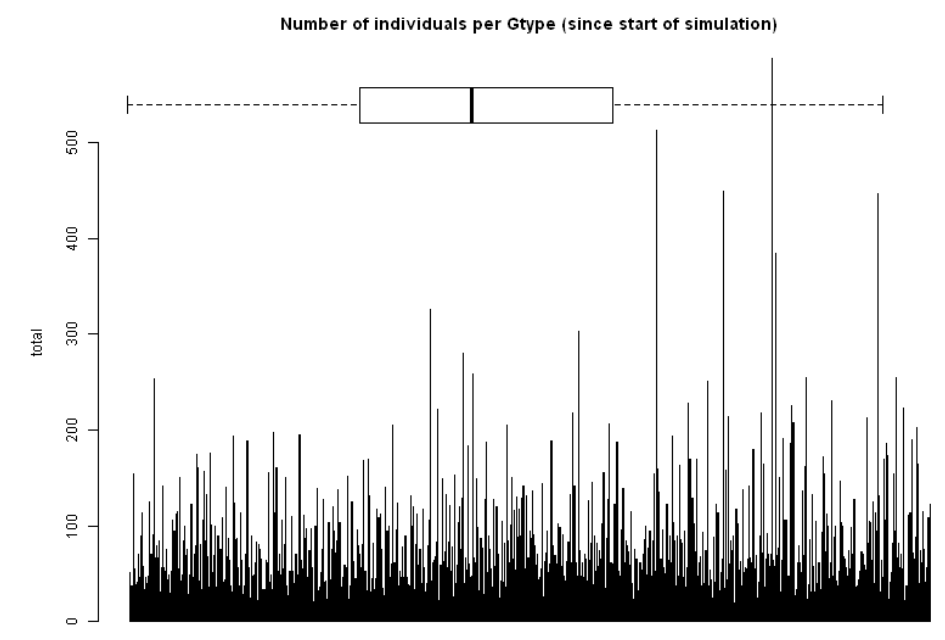

Figure 8.8: Entropy of the community. The graph illustrates the cumulative frequency of genotypes present in the community since the start of the run.

One important index to measure diversity is the Pielou's evenness index, which allows to determine how distributed the species are [177]. For instance, if there are 10 rats and 4000 foxes, the community is far from even. This is given by Eq. 8.1, where $J^{\prime}$ is the evenness factor, ranging in the interval between 0 and 1 ; the higher the value obtained the less variability there will be between the species. $H^{\prime}$ denotes the number derived from the Shannon diversity index and $H^{\prime} \max$ is the maximum value of $H^{\prime}$ equal to $\ln S$ [177].

$$
J^{\prime}=\frac{H^{\prime}}{H^{\prime} \max }
$$

Shannon's index for diversity [219] measures the richness of a population's heterogeneity. This is a measure introduced by Claude Shannon to determine the entropy in strings of text [217]. This is given by Eq. 8.2, where $H^{\prime}$ measures the richness of the biodiversity in a community, $R$ the total of individuals and $p i$ is the proportion of individuals belonging to the $i$ th species.

$$
H^{\prime}=-\sum_{i=1}^{R} p i * \ln (p i)
$$

High values of $H^{\prime}$ would be representative of more diverse communities. A community with only one species would have an $\mathrm{H}$ value of 0 because $p i$ would have the value 1 and is multiplied by $\ln (p i)$, which would be equal to zero. The final result of 8.086999 shows that the community in WisLM is rich in diversity. 
Departing from that value, the evenness for the day in question was found to be 0.001670297 which, considering the above reference values, is meaningful of a distributed community in WisLM.

\section{Spatial Distribution}

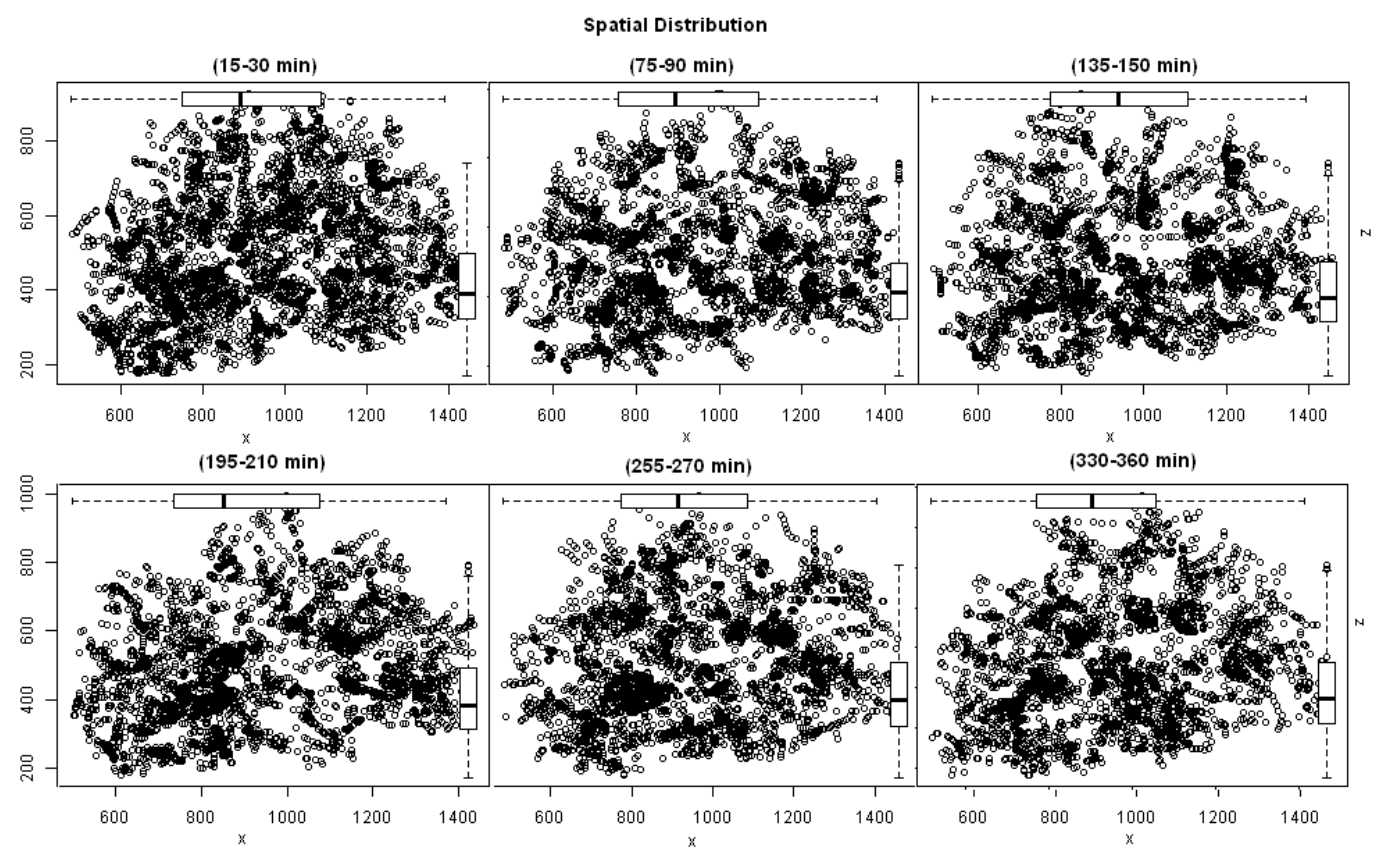

Figure 8.9: Spatial distribution in time. At intervals of 15 seconds, a snapshot of the system was captured during a period of 6 hours (one day of exhibition). Each dot represents the location at that particular moment of one individual. The variables $\mathrm{x}$ and $\mathrm{z}$ stand for the coordinates in the Cartesian horizontal space. Each graph illustrates a frame of juxtaposed 15 minutes of execution.

In order to study the spatial distribution, the position of each individual was recorded in time as a way to capture the distribution of the individuals in the space. Fig 8.9 illustrates the space occupancy in equal periods of 15 minutes each. Each of the frames is composed from the juxtaposition of the coordinates from the positions occupied by individuals, captured every 30 seconds and during a period of 15 minutes. The empty areas next to the boundaries of the frames denote the irregularity of the surface of the world, which is in the format of an island.

The graph shows that, far from a uniform distribution, the population keeps changing, shifting local attractors, thus occupying the space differently throughout the day. This is the result expected from an ecosystem's dynamics where these local attractors are emerging structures resulting from the self-organization of the individuals in their natural behaviour and struggle 
for survival.

\section{Heterogeneity of Actions}

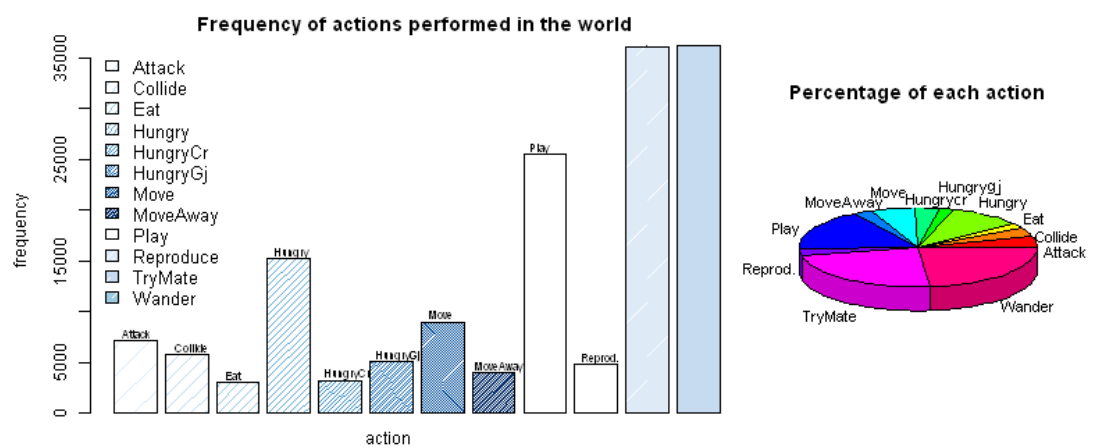

Figure 8.10: Heterogeneity of actions performed. The graph illustrates the number of times each of the actions was performed. At intervals of 15 seconds, the system recorded what each individual was doing in that moment of time. The heterogeneity of the actions performed is represented from left to right by Attack, Collide, Eat, Hungry, HungryCr (search for Carcasses), HungryGj (search for other creatures), Move, MoveAway, Play, Reproduce, TryMate, Wander.

The spontaneity of the actions performed by the individuals in the world was equally quantified. A histogram was produced showing the number of times each possible action was performed. Fig 8.10 shows that the results are distributed unevenly. The individuals spend their time mostly moving from one position to another. Notice that Move, Move Away, and Wander are separate categories. The main motivation is clearly Try to Mate, but it is interesting to see that the effective reproductions are quite low comparatively. This might explain the high level of Play. Again, Hungriness appears divided into three different categories, Hungry - in abstract, HungryGj - desire to eat another living creature, HungryCr - desire to eat a dead corpse. Again, the effective actions of Eating are quite low comparatively to the level of Hungriness generated. Here, it needs to be taken into account that the individual will most probably have to fight for its food.

Overall what this graph illustrates is that the behaviour observed in WisLM is far distant from what would be expected in a random uniform distribution. Clearly the CE produces interesting and motivated behaviours that are far from random. 


\section{Stability of the population density}

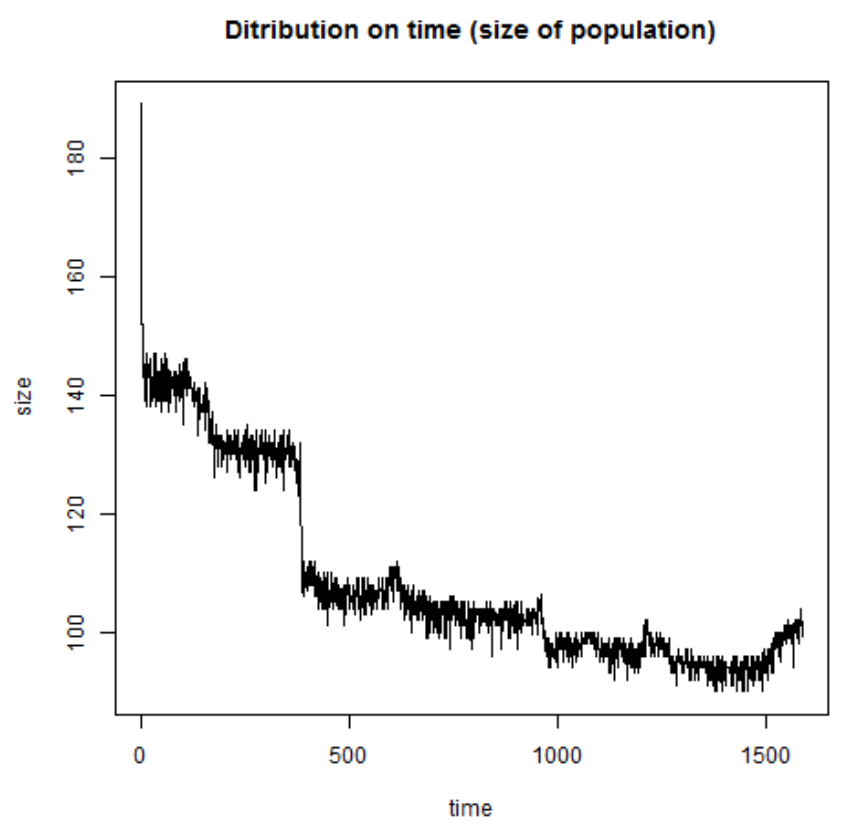

Figure 8.11: Population density. The graph depicts the temporal evolution of the demographic dimension of the community. Each unit in the time scale (the abscissas) denotes 15 seconds. The vertical axis denotes the number of inhabitants.

Fig 8.11 is a map that illustrates the evolution of the density of the community throughout the temporal frame of the run. It is clear that, as expected from an ecosystem's dynamics, fluctuations do occur, however these are mild and controlled with the population number converging towards safe and emerging attractors.

There are four distinct and demarcated steps. The initial population rapidly drops to about 140 inhabitants. Then it remains there for about half an hour to drop slightly to about 130 individuals, a density where it stays steady for another period of forty five minutes to one hour. It then drops again dramatically to values around 110, with small fluctuations being observed during the next period of about two hours. Then the population density drops again to the final floor, around 100 individuals, where it remains with small variations until the end of the day, when it rises again slightly to a value around 110. Overall the graph seems to indicate a tendency for the density to lower, perhaps even to extinction. This negative force is opposed by the stability algorithm (discussed in the chapter 6) that is able to successfully maintain these numbers up in stable levels; the population density was always above the minimum of 90 , and clearly below its maximum of 200 . 


\subsection{Discussion and future work}

In this thesis, the visualization of the internal dynamics of a functioning ecosystem is proposed as a way to structure and coordinate the animation of a population of humanoid avatars. The $\mathrm{CE}$ is proposed as an AI technique for behavioural animation of NPCs. This is exemplified with a population of NPCs simulating conversational behaviour in the virtual world WisLM. This discussion offers a critical analysis to this current implementation. The present chapter describes the implementation of such a method of production of behavioural motivations and consequent actions.

To build this model of AI, a set of established and efficient techniques for the agency of populations (Holland's classifier system, Jones' hormone-framework and Saruwatari et al's model with dietary specifications) was put together. This model of agency builds upon the architectural solutions implemented in my previous works. These systems have become successively more ambitious and complex. In $x T N Z$ [19], the mechanism for agency was a simple rule-based system. By contrast, in Senhora da Graça [21] a classifier system was used, a system that draws on McCormack and Holland's previous work [158, 119]. This is a system of sensors and actuators where actions are rewarded according to the consequences they have produced. A similar classifier reappered again in Vishnu's [23], only this time it was coupled with a system that allows dynamic hierarchies to be established between the individuals, by means of a generative food chain (inspired in Saruwatari et al [213]). In WisLM, these two techniques (classifier and dynamic food chain) reappeard again in the architecture, yet an extra layer of hormones (drawing on Jones' [129] work) was added to the implementation as part of the sensor mechanism.

Reasons to use a classifier and its advantages over other models of agency The complexity of the system could, however, have been greatly simplified. This model of agency, using a classifier system, has a complex implementation. However it also offers a number of significant advantages over other traditional models.

Rule-based systems (like $x T N Z$ ) require a much simpler implementation, but as discussed in chapter 3, a classifier is a method of reinforcement learning. This class of models provides far more interesting behaviours that are characterized by the adaptation of behaviours to a local context as individuals find it to be more beneficial/prejudicial to perform certain actions rather than others.

Additionally, in the classifier, an intermediary layer is added between the two layers of sensors and actuators. The priority of any given message that is triggered by the sensors needs to increase (by repetition of stimuli) before it activates the associated actions. As a 
consequence, a cybernetic delay exists between action and reaction, desire and realisation. This is arguably more surprising to the spectator and closer to the workings of life, where one may sometimes be hungry but is able to postpone the reactive action of eating until some other prioritary tasks are complete.

The ability to learn and adapt, and the possibilities for delays between action and reaction are also present in other techniques of reinforcement learning, such as Neural Networks. These have been used successfully in works like Polyworld [257]. The implementation of a Neural Network is, however, of greater complexity than the classifier used here. Additionally, due to their 'black-boxed' nature (the entanglement and interdependence of the nodes in the intermediary layer), Neural Network are harder to reverse-engineer when we need to fix undesired behaviors.

Consideration on the present implementation One aspect that I went on to inspect in detail about the present implementation was the advantage of using the hormonal system. Indeed in Vishnu's dance of Life and Death [23], the hormonal system was nonexistent and the behaviours were generated without this extra level of complexity. For the purpose of analysing the impact of removing the hormones, I changed the system to be regulated by three variables: energy, proxemia ${ }^{2}$ and joyfulness. This was in order to remove the hormonal system and still preserve an equivalent level of variety in the system. These two extra variables (proxemia and joyfulness) trigger actions that result in being more or less gregarious and reproductive, or playful, respectively. Table 8.3 shows the percentage of the times that each action was performed. The system was run 10 times, capturing at intervals of 30 seconds what each individual was doing, what action he was performing. Columns 2 and 3 show the median and standard deviation of these runs for the original algorithm used in WisLM. Table 8.3 (columns 4 and 5 - 3 variables), depicts the median when using three variables instead of the hormones. The results show that indeed the heterogeneity and the actions performed maintain identical levels to the original system (columns 2 and 3) and furthermore the percentage is in all cases similar to the model used in WisLM. This seems to indicate that, besides the biological association and metaphor, from the point of view of heterogeneity there is no obvious and immediately visible advantage to the hormonal approach rather than a simplified version. But this has forced me to use biologically implausible variables, such as the one for joyfulness and the second for gregariousness.

\footnotetext{
${ }^{2}$ Proxemia is a subjective concept introduced by anthropologist Edward T. Hall in The Hidden Dimension to describe different zones of comfort related with the proximity between the individuals.
} 


\begin{tabular}{|c|}
\hline Action \\
\hline Atack \\
\hline Attempt_Mate \\
\hline Collide \\
\hline Eat_Gajo \\
\hline Hungry \\
\hline Move \\
\hline Move_Away \\
\hline Play \\
\hline Reproduce \\
\hline Rotate \\
\hline Hungry_Carcassa \\
\hline Hungry_Gajo \\
\hline Wander \\
\hline
\end{tabular}

\begin{tabular}{|c|c|}
\hline \multicolumn{2}{|c|}{ Normal } \\
\hline Median & S.D \\
\hline 4.40 & 0.001420589 \\
\hline 23.63 & 0.041082177 \\
\hline 3.47 & 0.000924933 \\
\hline 1.84 & 0.000888886 \\
\hline 10.11 & 0.001884429 \\
\hline 5.34 & 0.001190365 \\
\hline 2.63 & 0.001557825 \\
\hline 16.08 & 0.026991158 \\
\hline 3.19 & 0.000822747 \\
\hline 0.40 & 0.000298083 \\
\hline 2.12 & 0.00065555 \\
\hline 3.37 & 0.011619446 \\
\hline 23.29 & 0.01242625 \\
\hline
\end{tabular}

\begin{tabular}{|c|c|}
\hline \multicolumn{2}{|c|}{3 Variables } \\
\hline Median & S.D \\
\hline 5.22 & 0.046660526 \\
\hline 22.71 & 0.02027092 \\
\hline 3.91 & 0.02620916 \\
\hline 2.09 & 0.016350021 \\
\hline 9.82 & 0.022603543 \\
\hline 5.57 & 0.023342281 \\
\hline 2.99 & 0.003523023 \\
\hline 16.48 & 0.012870568 \\
\hline 3.03 & 0.006055738 \\
\hline 0.37 & 0.001810103 \\
\hline 2.09 & 0.00806394 \\
\hline 3.21 & 0.005990928 \\
\hline 22.51 & 0.024696783 \\
\hline
\end{tabular}

\begin{tabular}{|c|c|}
\hline \multicolumn{2}{|c|}{ Only energy } \\
\hline Median & S.D \\
\hline 0.54 & 0.007871115 \\
\hline 16.32 & 0.101237441 \\
\hline 1.88 & 0.003434491 \\
\hline 0.07 & 0.000925708 \\
\hline 10.89 & 0.011178303 \\
\hline 1.57 & 0.038344527 \\
\hline 0.71 & 0.007159835 \\
\hline 50.12 & 0.356480959 \\
\hline 1.15 & 0.021713912 \\
\hline 0.10 & 0.004940925 \\
\hline 5.13 & 0.003004189 \\
\hline 5.20 & 0.004491342 \\
\hline 15.66 & 0.151640898 \\
\hline
\end{tabular}

Table 8.3: Median, resulting from 10 different runs, of the percentages of each of the actions performed using one of the three methods for generating action triggers: $i$ ) with a hormonal system, as in WisLM - columns 2 and 3; ii) without the hormonal system, but three regulatory variables (energy, proxemia and joyfullness) - columns 4 and 5; and iii) with just the energetic level - columns 6 and 7.

Later, I removed these two extra variables from the system, so only energy was used as a regulator. Two energetic thresholds trigger the behaviours: $i$ ) below a certain value the individual becomes hungry as before; $i$ ) above a certain limit the individual becomes playfull; iii) this is only up to a certain point, since above a second threshold, it becomes sexually active. Table 8.3 (columns 6 and 7 - Only energy) shows that this creates an immediate impact and changes the results drammatically. Play becomes now, by far, the action that is most perfomed. Attempt to Mate and Wander are still performed a substantial number of times, but in a smaller dimension. The results indicate that using this last strategy, the system becomes comparatively more monotonous for the viewer and generates less complexity and associated variability.

Regarding the extra complexity added with Sarawatari et al.'s system, I could have opted for the classical system composed of two single trophic levels with a population of consumers feeding exclusively from a population of producers. However Saruwatari's methodology provides for emergent multilevel food chains, which is far more interesting. On the one hand, this provides for niche formation, and on the other hand, it is richer from a metaphorical point of view. Rather than colonials preying on servants, this system widens the hyperbole bringing the economic system to the core of the interactions. Instead of a black and white portrait of the situation, we end up with gray levels where consumers live off the energy (the currency) not only from producers but from other consumers situated at lower levels, which is more accurate 
historically with the anthropological scene represented (by the same token, I should think of a third version where some producers may prey as well on other producers).

Other algorithms of crowd animation These features contrast in a number of ways with the standard alternative techniques for animation of crowds and groups presented in chapter 2. One clear disadvantage presented by the present method is that it is not computationally tractable for large crowds. One other aspect that needs optimization is the unsophisticated way the algorithm handles locomotion and collisions. Methodologies using the Cellular automata approach, for instance, define rules for behavior, which are dependent on the velocity, flow and level of density of the vicinity of each individual. On the other hand, social forces have movement and interactions defined by a set of equations, which also take the environment (physical obstacles and other agents) in consideration. These equations might be expanded to accomodate other aspects we may want to see modeled. These methodologies focus on ambulatory features of the crowd and its structure at the macro-level (crowd). The CE by contrast offers the advantage of an intrinsic level of heterogeneity and spontaneity of the behaviours of the crowd in the micro-level (groups and individuals). Another advantage of this over other models focusing on the micro-level of the crowd, in particular those simulating conversational behaviour (such as [186]), is that the visualization is not random but is indeed showing the internal states or stages of the trading of a currency (in the form of energy). The conversation the characters exhibit is indeed far from arbitrary. The movements in space and the animations of their gesticulations indicate the particular states of their trading activity. This might be relevant for specific design requirements, for instance in a simulation of a market-place. Other advantageous aspects are the typical fluctuations of population numbers offered by CEs, leading to a constant flux of entries and exits on the scene (due to births and deaths). This is not so evident in the instantiation of this model in this particular virtual world (WisLM) because the space is far too vast, but again, on some occasions in smaller places like interior scenes, this approach might be more relevant rather than a mere static population density.

The future Combining a crowd approach (based on particles) and a genuine ecosystem approach, such as the one discussed here, might offer new domains of exploratory possibilities. Further exploration on hybrids where the ecosystem paradigm is combined with a human crowd behaviour approach could have relevance to NPCs in computer games, particularly in MMOs where maintaining scripted behaviour for hundreds of NPCs is a big overhead. Articulating the $\mathrm{CE}$ with other existing strategies can only provide for a stronger technique. From existing implementations of CEs and crowd models, we can identify a number of aspects 
that could advantageously be introduced in the present model.

i) Economical model First and foremost is the naming and metaphor at play. Rather than predators, prey and food chains, we could equally be talking about a purely economical model. Echo [97] provides a model where multiple fountains of resources provide different types of resources. This could be explored so that individuals would have to capture resources of a certain type - in exchange for some useful work and energy expenditure. As individuals would need combinations of different resources to produce energy, these would have to be stored and traded with other members of the community. The obvious advantage is to avoid the problematic politics of equating species and human groups, which was interesting in discursive terms in the present work but can be avoided in other more general applications.

ii) Emergence of a moral system The second aspect I would like to see explored is peer pressure. When an action is performed, the members in the close vicinity could reward the individual (with some units of a resource for instance, or some measure of popularity that could be of reproductive advantage) based on a system of beliefs - such as on how benefitial this action was for each of them. For instance, buying all available stock of resources of type A might not be very 'useful' in terms of popularity if the neighbours also need that particular resource. On the contrary, giving away its own resources is a bad strategy in the eyes of selfpreservation, but might actually be well interpreted by the neighbours and thus be positively rewarded. Game theory has a strong tradition in this area of research and some good examples exist, such as Thébaud and Locatelli's work on the simulation of spontaneous order and peer pressure in the collection of driftwood in the shoreline after a storm [236].

iii) Navigation using layers and $A^{*}$ Existing models provide alternative mechanisms for locomotion, more efficient that the one in WisLM. Shao and Terzopoulus [218], for instance, implement a multi-layered approach that combines a reactive lower level to avoid basic collision with other objects, and a topological map composed of a hierarchy of grids: one for the geometry of the environment, a second for static objects and a third to list all the pedestrians. These maps are used to calculate the optimal long path to the objective using $\mathrm{A}^{*}$ and adjust short trajectories when the ideal path is occupied.

iv) Breaking down the animation and a language of movements One aspect to take notice of in the current implementation, is the small-number of pre-generated movements as well as the lack of sophisticated blending, which is limiting and hides the richness that could 
be effectively reached. An increased set of animations would make the system more flexible. Currently, the system has a one-to-one relationship between actions and animations. When an individual 'attacks' prey, one animation is played. However a fuzzy logic system could be implemented to activate different animations depending on the levels of energy from predator and prey.

To explore this potential further, it would be interesting to consider gestures and poses which reflect the internal states of the avatars. This could be enriched with a wider set of animations, which might reflect such nuances. Also, in contrast with this current deterministic approach of defining a limited set of animations, it would be interesting to break the animations into smaller bits. The use of the CE in WisLM was mainly for the inherent quality of indeterminacy that is associated; the $\mathrm{CE}$ was explored as a generator of heterogenous behaviours. In terms of emergence and novelty, this work would become richer with the incorporation of elements of a language of movements on which the CE could act. This would create the conditions for procedural movements with potential emergence of unexpected movements, perhaps rich in subtleties more in accord with a virtual evolutionary paradigm.

v) Finite state machines to activate sequences of animations One criticism we can put to the present work is that the avatars appear and disappear as if by magic, into thin air. If on the one hand this was important for the reading of the artistic project (individuals being removed from the world as a result from the predatory interactions), on the other hand it breaks with the illusion of a realistic crowd. One easy way to circumvent this issue is to create an upper level of operation where the character, after being born, instead of appearing next to its parents, emerges opening the door of the closest building, and in a similar process moves to an arbitrary building in order to die out of sight.

This would be easily implemented with the introduction of finite state machines (FSM) like in the work of Musse et al. in PetroSims, described in [234]. This requires a leap in the current reasoning in order to consider the classifier as just a module/state in a FSM. Actions such as 'eat' could be the necessary trigger to activate not one animation but rather a state that implements multiple behaviours and animations. One example is when the individual gets to the fountain of resources A to fill up on a deposit of that resource. When this event happens, the action 'eat' creates a transition of state, activating not one animation but a state of the FSM that is composed of a sequence of animations: 1) waiting in the queue until its own deposit of resources is filled; 2) then moving to the theatre; 3) locating seat number $\mathrm{x}$ and seat; 4) seating until end of show; 5) moving away; 6) reactivating the classifier.

The animation of Pompeii by Maim et al. [149] provides one example of the possibilities 
this approach entails when combined with semantic objects in the environment. Semantic objects are objects that, besides their visual information, also contain meta information. In Pompeii, these objects trigger FSM states with predefined animations, such as 'look at the shop-window'.

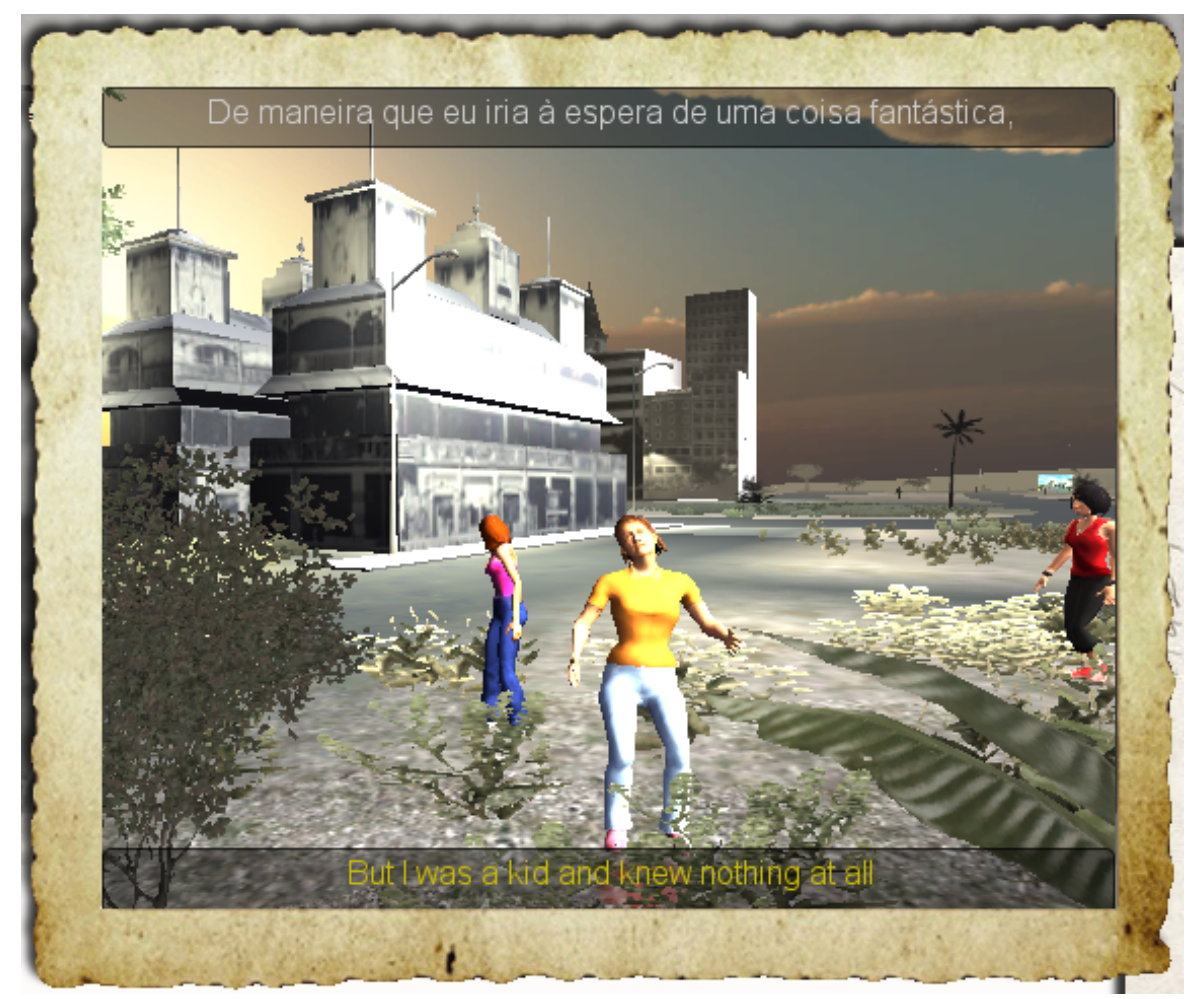

Figure 8.12: Detailed screen-shot from the website capturing the moment a storyteller tells her account with visible subtitles in Portuguese (top) and English (bottom).

\subsection{Final notes}

This chapter was organized into three main sections: The first of these described the motivations for the artwork Where is Lourenço Marques? (A more detailed description of the motivations is available at [16]). Section two presented details about the CE, describing the AI model that animates each of the individuals in the community of humanoid avatars. Finally, in section three, a discussion on the $\mathrm{CE}$ as a generative technology was presented: a critical analysis to this specific implementation is produced.

Traditional EvoArt is characterized by a process of translation (interpretation of symbols) between the Gtype and the Ptype. The approach described here, rather than limiting this 
methodology to the Gtype, also visualizes the ephemeral states of the individuals in the dynamics of an ecosystem. The behaviours of the individuals during their normal activity, their primal movements and actions, such as attack, flee, mate, prey, etc. are taken as syntactic elements during a process of interpretation. For instance, the action of 'eating' occurring at the ecosystem level might be translated in the semantic level as the animation of a wild animal chewing a carcass in the virtual world. Similarly the syntactic element 'chase' might be converted as the animation of the same animal running. However, as it happens with the original Gtype-Ptype paradigm, this process is also open to creativity, and the linearity and distance of the process of translation are subjective to the artistic interpretation of the author. For instance, the action 'attack', rather than the animation of an animal fighting, might instead correspond to a specific sound being played in a orchestra or even to a specific movement being choreographed to be performed by a dancer.

This research explores these ideas in the animation of a population of humanoids in a virtual world. The investigation has been driven by the design specifications for the artwork WisLM, which required the existence of an autonomous population of humanoid avatars to play the role of storytellers in the virtual world. However, the same specifications also required the behaviour of this community of humanoids to be a consequence of the dynamics of an ecosystem where individuals would prey on each other. A computational ecosystem - a system of agents organized in a hierarchical structure (of a food chain) and trading token units (of energy and biomass) - generates these dynamics. One of the aspects of the complexity of crowd interaction is the spontaneity as groups of individuals form and the heterogeneity of gestures exhibited during their conversations. A population of gregarious NPCs showing some of these spontaneous and conversational behaviours was modeled. This approach took advantage of the fundamental properties of CEs: that taking up the variety and spontaneity of the elementary behaviours, the autonomy and self-organization of the individuals generates everchanging heterogenous patterns on the global scale of the community. In other words, to build up WisLM, I have drawn on the fact that the CE is, in essence, a generative methodology which may be applied to general purpose NPCs. 


\section{Conclusions}

This manuscript presents a study on the artistic production that is characterized by the use of computational ecosystems (CEs) as a main foundation. A small but essential set of works map out the territory of Evolutionary Art (EvoArt) with collections of articles describing works and respective modes of production: Creative Evolutionary Systems edited by Peter Bentley and David Corne [36], and The Art of Evolutionary Art, edited by Penousal Machado et al. [210], and the recently published Virtual Worlds, edited by Stefan Bornhofen et al. [49]. While providing a good image of the field, these works put a great emphasis on the technical implementation of the artefacts. Metacreations however, authored by Mitchell Whitelaw [251], provides a more comprehensive analysis to the field, with a study that includes the cultural framework of the practice. The chapter on Cybernatures and an article from Alan Dorin in the The art of Evolutionary Art started to delineate a line of research that refines these studies to the domain of EvoArt using CEs. The aim of the present investigation is to keep on that track and examine the CE in Virtual Worlds (VWs). In this document, it is argued that the remediation of these systems in VWs widens the scope of their technical and artistic language.

As discussed in chapter 2, Rosalind Krauss argues the term 'technical support' as the set of ideas informing an artistic practice. The execution of the material artefacts appears subsidiary to the conceptual framework. In the case of Ed Ruscha, the technical support of his painting is the automobile. In Sophie Calle, it is photo-journalism [135]. By the same token, it can be suggested that the foundation of ALife, and EvoArt in consequence, is not rooted in the set of techniques used in the construction of the artefacts, but rather on the ideas informing the practice.

One of the notions that emerge from this reasoning is a separation between the narratives of life usually associated with CEs and the techniques used in their construction. Abstracted as a generative technique, the $\mathrm{CE}$ can be looked at from the point of view of a generative model for animation of general purpose non-player characters (NPCs). This was the project I have set out to interrogate with this investigative process, to examine the $\mathrm{CE}$ as a generative technology for heterogeneity and spontaneity that may be used in the animation of general purpose NPCs.

The hypothesis of CEs as pure generative techniques is informed by the recent branch of 
Evo-Artists using CEs to explore the sonic domain (e.g [90, 128]). These develop works where the ephemeral states of the components of the ecosystem appear as audible format. An investigation unfolded as to whether or not the animation of general purpose NPCs in VWs could be established upon these ephemeral states and the overall internal dynamics of the ecosystems.

Additionally, this manuscript offers a study in one of the most underrated problems affecting these systems. This is the persistence of the populations that form the virtual communities. Four methods were investigated, and as a result a heuristic was suggested as a way to promote mild fluctuations in the population density. This heuristic was then implemented in the virtual world Where is Lourenço Marques? (WisLM), an ethnographic artwork which functions as empirical evidence of the practical usefulness of both the heuristic suggested and the CE as a generative technology for animation.

Where is Lourenço Marques? The work WisLM functioned as a catalyst and main motivation for this research. This artwork required a community of humanoid NPCs to play the role of 'storytellers' in the virtual world. By imposition of the design-specification, the actions and behaviours of this population in the virtual space had to be driven by the dynamics generated within an ecosystem. This strict and constraining 'brief' has generated a series of questions about the implementation of such a project. These are situated both in the technical and aesthetic realms, and this manuscript synthesizes the consequent analytic work. These developments allowed the use of a CE as a model for AI in the animation of the community of humanoid NPCs in the artwork WisLM. Consequently, this work is a critical piece in this research as it appears in the beginning of the investigative process and at its closure: it functions as the catalyst for inquiry and is the causa finalis.

Evolutionary Art WisLM is suggested as an instance from a new generation of Evolutionary art interested in expanding the style's dialectic territory, traditionally much restricted to its evolutionary/generative tenets. The generative aesthetics imposes the existence of a recurrent system of rules as the building foundation for the creation of the artefacts. With EvoArt, a sub-genre of generative art, this system informs on evolution by Natural Selection associated with Mendelian Genetics. In turn, CEs are a singular case of EvoArt where the works are as well characterized by identical Neo-Darwinian precepts, but essentially these works can be described as dynamic ecosystems formed by autonomous individuals.

EvoArt draws on the paradigms provided by Genetic Algorithm, an optimization technique originated in Evolutionary Computation (Chapter 3). The abstract methodology of the Genetic 
Algorithm entails a pool of individuals competing with each other for the perpetuation of their genes. Each individual is codified using a genetic blue-print (the Gtype). Typically during a process of reproduction, the 'fittest' individuals generate new individuals. This is a process that combines the progenitor's genomes mixing the information contained in the respective Gtypes by means of operators of crossover and mutation. The recurrent use of this procedure generates exploratory 'drifts' through the genetic landscape, this being the reason why this methodology is considered an optimization technique.

Drawing on this exploratory potential, the pioneers of Evolutionary Art have helped to establish an artistic paradigm by their use of this process for the generation of still imagery (e.g [237, 211]. The Gtype-Ptype paradigm entails the conversion of a 'syntactic' element containing the information about the individual (the Gtype) into the 'semantic' interpretation of that same information (the Ptype). These pioneers have shown that this interpretative process was open to individual creativity from the artists and that the technique could be employed for the exploration of image spaces (Chapter 3).

However, the CE offers a new approach to this paradigm. The populations are now communities formed by dynamic and evolving individuals. These are not static objects or images as happens with the individuals in the Gtype-Ptype, but rather autonomous agents actively struggling for survival and perpetuation of their genes. The systems have intrinsic dynamics, and these are generated by the actions and movements of the agents roaming the space in search for energy or sexual partners. There is also a change of scale regarding the object of interest in these two approaches: whereas the pioneers were focused on the individual instances, now this focus of attention shifts towards the system, the domain of the community itself.

Ecosystems as Al models As becomes clear from the panorama presented in AppendixA, the themes and processes in CEs haven't changed much in the last two decades. Recently however, some exploratory works appeared to challenge this relatively quiet landscape by means of exploring for aesthetic purposes the dynamics of the system itself. These are works stemming from the audio sphere of the practice. Granular orchestrations are generated by the community of individuals that form the CE. In Wakefield and Ji's work, the populations 'sing' their genomes [128], whereas in Eldrige and Dorin's work, they sing their internal states (energetic level) [90].

This thesis has built on this more recent approach with the intention of expanding on it. The $\mathrm{CE}$ is argued in the animation of general purpose non-player characters in virtual worlds. At the level of their dynamics, these systems are open to the possibility of processes of interpretation similar to the ones from the Gtype-Ptype paradigm. The ephemeral actions occurring at 
the level of the ecosystem, such as 'eat', 'attack', etc. might be considered syntatic elements in a process of transformation to a semantic level. In a similar way to what occurs in the Gtype-Ptype paradigm, this process is also open to artistic creativity.

This interpretative step is addressed by Katherine Hayles, who identifies a functional gap between the material processes occurring at the level of the hardware and software, and their reading. As she argues, it is a process of narrative that fills in this gap (Chapter 5). As a consequence, the instrumentalisation of these processes in the animation of non-player characters in virtual worlds is argued. Drawing on this gap, the syntactic element 'eat' might be translated in the semantic level by the primal animation of an animal covered with blood and eating some other animal fiercely killed or instead to be translated more freely, for instance, by the animation of a humanoid dancer performing a specific body gesture in a dance movement. As with the predecessor paradigm Gtype-Ptype, the distance and linearity of the process of conversion are open to creativity and artistic expression. In Vishnu's, for instance, this process was demonstrated through the animation of a community formed by humanoid dancers, and in WisLM this was explored in a community of gregarious conversing individuals.

As such, this thesis is advocating what can be described as the 'remediation' of the technology of the $\mathrm{CE}$ in virtual worlds. In a loose understanding of the concept of remediation, as proposed by Grusin and Bolter [46], in the process of remediation it is said that the 'old' technology is reshaped and may even gain and create new social uses. Incorporated into virtual worlds, the $\mathrm{CE}$ maintains the potential to tell the traditional narratives about communities of bacteria living in a chemostat, or animals in the jungle eating each other while struggling for energy and the self-perpetuation of their genes. However, this incorporation also gives something additional since it opens the process to a broader scope of other narratives, such as the ones explored here: humanoids from different social classes in conversational behaviour while trading units of currency, or even other abstract interpretations, such as performers dancing wild choreographies. The $\mathrm{CE}$ is thus argued as a generative technology for heterogeneity, spontaneity and novelty in virtual worlds. It is a technology that is fundamentally open in terms of the creative uses that artists can make out of it, and it is rich in term of the plasticity entailed by the inherent levels of expressiveness.

Organization of the manuscript This manuscript was divided into two major sections, the first of which contextualizes the work, engaging in a theoretical debate on aspects that directly influence this practice and the second delivers empirical developments that address the questions raised. It started with a literature review where CEs were interrogated as technical and cultural products. This was divided into four chapters, which unfolded in a way as to map 
and orient the main argument of the thesis.

At first, these artefacts were examined in their materiality. This was to acknowledge techniques for the construction of the system in WisLM. For this project, a teleology of Evolutionary art was produced, scrutinizing techniques that have established the framework of this tradition. The fundamental methods that are involved in the construction of the systems were identified. As a result, three paradigms were suggested as informing the development of CEs (Chapter 3): a) the Gtype-Ptype, b) the cellular automaton; and c) the co-evolution in multiagent-systems.

Following, a summary of the cultural framework informing this praxis was laid out. This was to identify the set of main ideas that inform the methodologies and processes. Evolutionary Art was first contextualized in historical terms as a sub-genre of Generative art, and as a style that informs in the post-war interest for systems, which was expressed artistically by the schools of op-art and kinetic-art. The twist introduced by Evolutionary Art in relation to GenArt is that the system is inspired by biological processes, in particular the neo-Darwinian interest in Genetics and Evolution by Natural Selection. This was then emphasized as informing the aesthetics of the production of CEs with a toolkit where bottom-up methodologies and ideas of material self-organization and emergence, borrowed from ALife, appear at the centre of practice and discourse.

Moreover, I have looked at CE modes of functioning through the analysis of these artefacts as cultural forms. This was to understand the systems of representation at play. A process of deconstruction was conducted in order to examine the processes involved in writing and reading them (Chapter 5), the processes at play when these provide context and artistic meaning. Semiotics played a key role in helping to situate the ephemeral events occurring at the ecosystem level (or ultimately at the machine level) as signifiers. These are part of a system of signs that may be decoded in multiple ways depending on momentary contextual maps of meaning. This discussion was useful in order to suggest an inclusive model of narrative. The term 'system narrative' was borrowed from Mitchell Whitelaw to describe a convergence of processes which simultaneously interfere and play their influence in the reading of the artefact. These processes consist of the agency of the population, the materiality of code, the physical artefact, the audience, and the 'interpretative framework' as proposed by Lizbeth Klastrup. Understanding the intricate interplay of this system is a critical tool for any artist aiming to operate with CEs.

The second part of the manuscript presented two detailed reports of empirical work aimed at answering and demonstrating particular aspects of the questions raised. Chapter 6 presents a work of analysis on the persistence of the populations in CEs. Chapter 7 and 8 lay out 
the motivations and the implementation of the work WisLM where the CE was used as an animation technology.

The persistence of the populations is one of the understated problems in the development of CEs. By tinkering and adjusting the parameters, developers usually manage to get relatively stable communities. However, the first study presented is an attempt to avoid this process of manual tinkering as it explores heuristics to manage population densities free of wild fluctuations. Chapter 6 explores some methodologies for the construction of systems where the population numbers fluctuate mildly and do not tend to reach either i) extinction levels or ii) exponential growth (in other words, persistent computational ecosystems). Overpopulation and massive-extinction are two recurring problems of these systems, and two different mitigation strategies were investigated: active insertion or deletion of individuals when critical densities are reached and inhibition of new insertions or deletions from being performed. The efficiency of these strategies was compared in terms of the minimization of interferences with the natural evolutionary course of the community.

Finally, Chapters 7 and 8, have described the work Where is Lourenço Marques?. The first of these chapters presented the motivations for the artwork and the reasons why such an unusual system like the $\mathrm{CE}$ was required as an animation tool. As such, this chapter has presented the main justifications for the research. This investigation was motivated in part by the design specifications of the artistic work, which required the animations of a humanoid population to be driven by predatory behaviours in an ecosystem. Predatory behaviour is used as a metaphor/allegory for the social conditions lived in that colonial city. In particular, the work demanded an AI model with agents organized in the hierarchical structure of a food chain while trading token units of energy and biomass as a way of promoting community dynamics. The CE was argued as a generative model that provides for such targeted complex environment simulations that are rich in heterogeneity and spontaneity.

Chapter 8, in turn, described the technical implementation of such work. A discussion was presented on the production of the visual effects of a community of gregarious individuals with small autonomous and interactive crowds (groups of 2 to 10 individuals, Figure 7.2). Furthermore, the CE driving the behaviours was described in detail. Part of this system is a module with the heuristics developed and discussed in Chapter 6. The chapter closes with an analysis of the behaviours generated by this algorithm in the final work that was displayed to the public in the exhibitions of 2012, namely Watermans Festival of Digital Arts and the Tin Shed space. In a way, this implementation suggests the usefulness of a) the heuristics suggested to generate persistent populations and b) the $\mathrm{CE}$ as a technology for the animation of humanoid gregarious NPCs. 
Closing Notes This thesis establishes a framework for the artistic production of CEs. Overall it can be said that global analysis unfolds in different areas of knowledge, providing multiple contribution that enrich both the technological and the artistic domains. The distinctive contribution to new knowledge provided through this research, practice and theory is the transition of perspective, which allows us to look at CEs pragmatically as generative technologies for the animation of general purpose NPCs in VWs. Dissociated from the traditional narratives of life, this study helps us to see the essence of the CE as a rich and open generative technology for heterogeneity, spontaneity and even novelty, that is adaptable to multiple contexts.

Furthermore, in the technical realm, the formalization of a new AI model for the development of populated virtual environments was suggested as a way to enhance the architectures of VWs (Chapter 8). Moreover, CEs were analyzed from the point of view of their persistence and a heuristic to address sharp fluctuations of population density was proposed (Chapter 6). In the aesthetic domain, this research has also provided an important contribution with a technical and cultural framework for the use of CEs as technologies for artistic practice. And finally, by means of the work Where is Lourenço Marques?, this research has provided new contexts for EvoArt practice by hybridizing generative practices with a perspective more centered on inter-human relations and the human stories that these works may represent or produce (Chapter 7 and Chapter 5), and consequently contributing to engage EvoArt with Relational Aesthetics in a richer way. 


\section{Bibliography}

[1] Memory of the Netherlands, http://www.geheugenvannederland.nl (accessed June 15th, 2013).

[2] Wired Magazine - http://www.wired.co.uk/magazine (accessed June 15th, 2013).

[3] Human Genome Project - http://www.ornl.gov/sci/techresources/Human_Genome/home.shtml (accessed June 15th, 2013), 1990.

[4] Lia's website, http://www.liaworks.com/livevisuals/about-visuals/ (accessed June 15th, 2013). 2013.

[5] Pet pet Park - http://www.petpetpark.com/ , (accessed June 15th, 2013), 2013.

[6] E. Aarseth. Cybertext: Perspectives on Ergodic Literature. The John Hopkins University Press, 1997.

[7] A. Adamatzky and M. Komosinski, editors. Artificial Life Models in Software. Springer-Verlag, London, 2005.

[8] C. Adami. Ab Initio Modeling of Ecosystems with Artificial Life. Natural Resource Modeling, (15), 2002.

[9] C. Adami and C. T. Brown. Evolutionary Learning in the 2d Artificial Life System "Avida". In R. A. Brooks and P. Maes, editors, Artificial Life IV: Proceedings of the Fourth International Workshop on the Synthesis and Simulation of Living Systems, pages 377-381. MIT Press, 1994.

[10] M.M. Al-Rifaie and M. Bishop. Swarmic Sketches Deploy Attention Mechanism. In P. Machado, J. McDermott, and A. Carballal, editors, Proceedings of the Second International Conference on Evolutionary and Biologically Inspired Music, Sound, Art and Design, EvoMUSART 2013, volume 7834, pages 85-96. Springer, 2013. 
[11] S. Allesina and A. Bodini. Who Dominates Whom In the Ecosystem? Energy Flow Bottlenecks and Cascading Extinctions. Journal of Theoretical Biology, 230(3):351-8, 2004.

[12] M. alRifaie. Information Sharing Impact of Stochastic Diffusion Search on Population Based Algorithms. PhD Thesis, University of London, 2012.

[13] Pardey Andres and Klaus Littman. Jean Tinguely: Retrospective. Instituto Valenciano de Arte Moderno (IVAM), 2008.

[14] M. Annunziato. The Nagual Experiment. In Proceedings of the First International Conference on Generative Art, 1998.

[15] M. Annunziato and P. Pierucci. Towards Artificial Societies. In Proceedings of the Third International Conference on Generative Art, 2000.

[16] R. F. Antunes. Where is Lourenço Marques?: A Mosaic of Voices in a 3D Virtual World. Leonardo Electronic Almanac, 18 (Touch and Go)(3):114-121, 2012.

[17] R. F. Antunes and F. F. Leymarie. Generative Narrative in Computational Ecosystems. In ISEA 2011, 17th International Symposium of Electronic Art, http://isea2011.sabanciuniv.edu/paper/generative-narrative-computationalecosystems.

[18] R. F. Antunes and F. F. Leymarie. Where is Lourenço Marques?, http://www.lourencomarques.net (accessed June 15th, 2013).

[19] R. F. Antunes and F. F. Leymarie. xTNZ - An Evolutionary Three-dimensional Ecosystem. In A. Barbosa, editor, Proceedings of the 4th International Conference on Digital Arts, Artech2008, pages 201-204, 2008.

[20] R. F. Antunes and F. F. Leymarie. Senhora da Graça, a Virtual World as Memorial. In Rosa Maria Pinho Oliveira, editor, Ciantec 2009 - III Congresso Internacional das Artes, Novas Tecnologias e Comunicação, Aveiro, Portugal, 2009.

[21] R. F. Antunes and F. F. Leymarie. Epigenetics as Aesthetic Instrument in a Generative Virtual Ecosystem. In L. Valbom, editor, Proceedings of the 5th International Conference on Digital Art ARTECH 2010, pages 172-176, 2010. 
[22] R. F. Antunes and F. F. Leymarie. Virtual Worlds as Art Practice: EvoArt Methodologies. In A. Seal, J. Bowen, and K. Ng, editors, VA London 2010: Electronic Visualization and the Arts, pages 271-277. British Computer Society, 2010.

[23] R. F. Antunes and F. F. Leymarie. Generative Choreography: Animating in Real Time Dancing Avatars. In P. Machado, J. Romero, and A. Carballal, editors, Proceedings of the First International Conference on Evolutionary and Biologically Inspired Music, Sound, Art and Design, EvoMUSART 2012, pages 1-10, 2012.

[24] R. F. Antunes and F. F. Leymarie. An Ecosystem Based Model for Real-Time Generative Animation of Humanoid Non-Player Characters. In L.P. Reis L. Correia and J. Cascalho, editors, 16th Portuguese Conference on Artificial Inteligence (EPIA 2013), LNAI 8154, pages 66-77. Springer, 2013.

[25] R. F. Antunes and F. F. Leymarie. Real-Time Behavioral Animation of Humanoid Non-Player Characters with a Computational Ecosystem. In R. Aylett et al., editor, 13th Conference on Inteligent Virtual Agents (IVA 2013), LNAI 8108, pages 382-395. Springer, 2013.

[26] Electronic Arts. Spore-website, http://www.spore.com/ftl (accessed june 15th, 2013), 2009.

[27] Electronic Arts. Sporopedia-website, http://www.spore.com/sporepedia (accessed june 15th, 2013), 2009.

[28] D. A. August and D. G. Andersen. The Garden: Evolving Warriors in Core Wars, http://www.angio.net/res/garden.html (accessed June 15th, 2013), 2011.

[29] J. Baal-Teshuva. Alexander Calder, 1898-1976. Taschen, 1999.

[30] S. Bajula, D. Pomerleau, and T. Jochem. Towards Automated Artificial Evolution for Computer-Generated Images. Connection Science, 6:325-354, 1994.

[31] Bandai. Tamagoshi - http://www.bandai.com/tamagotchi/ (accessed june 15th, 2013).

[32] B. Banerjee, A. Abukmail, and L. Kraemer. Advancing the Layered Approach to AgentBased Crowd Simulation. In Proceedings of the 22nd Workshop on Principles of Advanced and Distributed Simulation, PADS '08, pages 185-192. IEEE Computer Society, 2008. 
[33] T. Barbalet. Sustainability and Entropy in Biota Live Podcasts archive, http://www.biota.org/podcast/live.html\#9 (accessed June 15th, 2013), 2008.

[34] M. Batty. Agent-Based Pedestrian Modeling. Environment and Planning B: Planning and Design, 28:321-326, 2001.

[35] B. Benes. A Stable Modeling of Large Plant Ecosystems. In Proc, of the ICCVG'02 International Conference on Computer Vision and Graphic, pages 94-101. Association for Image Processing, 2002.

[36] P. Bentley and D. Corne. Creative Evolutionary Systems. Academic Press, 2002.

[37] P. J. Bentley and D. J. Corne. An Introduction to Creative Evolutionary Systems. In P. J. Bentley and D. J. Corne, editors, Creative Evolutionary Systems, pages 1-76. Academic Press, 2002.

[38] H. Bergson. Matter and Memory. Dover Publications (republication from 1912 edition MacMillan), 2004.

[39] R. Berry, W. Rungsarityotin, and A. Dorin. Unfinished Symphonies - Songs of $31 \backslash 2$ Worlds. In Bilotta et al, editor, ECAL 2001 Artificial Life Models for Musical Applications, pages 51-64. Konferensbidrag, 2001.

[40] V. Bertalanffy. General System theory: Foundations, Development, Applications. G. Braziller, revised edition 1976, 1968.

[41] C. Bishop, editor. Participation. The MIT Press, 2006.

[42] D. Bisig and T. Unemi. Cycles - Blending Natural and Artificial Properties in a Generative Artwork. In Proceedings of the Generative Art Conference (GA XIII). Milano, Italy. 2010.

[43] T. Blackwell. Swarm Granulation. In J. Romero and P. Machado, editors, The Art of Artificial Evolution: A Handbook on Evolutionary Art and Music, pages 103-122. Springer-Verlag, 2008.

[44] M. A. Boden and E. A. Edmonds. What is Generative Art? Digital Creativity, 20(1 \& 2):21-46, 2009. 
[45] A. Bogdanovych, K. Ijaz, and S. Simoff. The City of Uruk: Teaching Ancient History in a Virtual World. In Y. Nakano, M. Neff, A. Paiva, and M. Walker, editors, Intelligent Virtual Agents, volume 7502 of Lecture Notes in Computer Science, pages 28-35. Springer, 2012.

[46] J. D. Bolter and R. Grusin. Remediation: Understanding New Media. MIT Press, 2000.

[47] C. Bornhofen and S. Lattaud. Outlines of Artificial Life: A Brief History of Evolutionary Individual Based Models. In Lecture Notes in Computer Science: Artificial Evolution, pages 226-237. Springer, 2006.

[48] S. Bornhofen, V. Gardeux, and A. Machizaud. From Swarm Art Toward Ecosystem Art. International Journal of Swarm Intelligence Research (IJSIR), 3(3):1-18, 2012.

[49] S. Bornhofen, J. Heudin, A. Lioret, and J. Torrel, editors. Virtual Worlds: Artificial Ecosystems and Digital Art Exploration. Science ebooks, 2012.

[50] N. Borriaud. Relational Aesthetics. Presses du réel, 2002.

[51] O. Bown. A Framework For Ecosystem-Based Generative Music. In Proceedings of the SMC 2009 - 6th Sound and Music Computing Conference, pages 23-25, 2009.

[52] O. Bown. Ecosystem Models for Real-time Generative Music. In G. Scavone, V. Verfaille, and A. da Silva, editors, Proceedings of the 2009 International Computer Music Conference (ICMC 2009), pages 537-540, 2009.

[53] O. Bown and J. McCormack. Taming Nature, Tapping the Creative Potential of Ecosystem Models in the Arts. Digital Creativity, 21(4):215-231.

[54] T. Broughton, P. S. Coates, and H. Jackson. Exploring Three-dimensional Design Worlds Using Lindenmeyer Systems and Genetic Programming. In P. Bentley, editor, Evolutionary Design Using Computers, pages 323-341. Academic Press, 1999.

[55] R. Brown, I. Aleksander, J. MacKenzie, and J. Faith. Biotica: Art, Emergence And Artificial Life. RCA Computer Related Design Research. Art Books Intl Ltd, 2001.

[56] J. Burnham. Beyond Modern Sculpture: The Effects of Science and Technology on the Sculpture of this Century. Penguin Press, London, 1968.

[57] D. Burraston and E. Edmonds. Cellular Automata in Generative Electronic Music and Sonic Art: A Historical and Technical Review. Digital Creativity, 16(3), 2005. 
[58] C. Burstedde, K. Klauck, A. Schadschneider, and J. Zittartz. Simulation of Pedestrian Dynamics Using a Two-Dimensional Cellular Automaton. Physica A: Statistical Mechanics and its Applications, 295(3-4):507-525, June 2001.

[59] R. Callois. Man, Play and Games. Free Press of Glencoe, 1961.

[60] M. Cardenas. Becoming Dragon: A Transversal Technology Study, http://ctheory.net/articles.aspx?id=639 (accessed June 15th, 2013), 2007.

[61] P. Cariani. Emergence and Artificial Life. In C. Langton, editor, Artificial Life II, pages 775- 797. Addison-Wesley, 1992.

[62] M. Carvalhais. Towards a Model for Artificial Aesthetics. In Proceedings of GA2010 XIII Generative Art Conference, 2010.

[63] G. Caughley. Directions in Conservation Biology. Journal of Animal Ecology, 63:215244, 1994.

[64] C. Chen and J. Hoyami. Autonomous Systems for Interactive Digital Art. In 10th Generative Art Conference GA2007, 2008.

[65] The MOCA collection. Frank Stella's Ctesiphon I http://www.moca.org/pc/viewArtWork.php?id=110 (accessed June 15th, 2013), 1968.

[66] The MOMA collection. Frank Stella's The Marriage of Reason and Squalot, II - http://www.moma.org/collection/object.php?object_id=80316 (accessed June 15th, 2013), 1959.

[67] P. Dahlstedt and M. G. Nordahl. Living Melodies: Coevolution of Sonic Communication. Leonardo, 34(3), June 2001.

[68] B. Damer. Avatars! Peachpit Press, 1997.

[69] B. Damer, K. M., F. Revi, T. Furmanski, and C. Laurel. Nerve Garden: Germinating Biological Metaphors in Net-based Virtual Worlds. In A. Adamatzky and M. Komosinski, editors, Artificial Life Models In Software, pages 67-80. Springer, London, UK, 2005.

[70] R. Dawkins. The Blind Watchmaker. Norton and Company Inc., 1986. 
[71] K. A. De Jong. Evolutionary Computation. MIT Press, London, England, 2006.

[72] N. de La Peña and P. Weil. Gone Gitmo, A chronicle of the development of Gone Gitmo, a virtual installation of Guantanamo Bay prision in Second Life, http://gonegitmo.blogspot.co.uk (accessed June 15th, 2013), 2007.

[73] G. Deleuze. Cinema 2: The Time-Image. University of Minnesota Press, 1989.

[74] T. J. Demos. Art After Nature. Artforum, pages 191-198, April 2012.

[75] A. K. Dewdney. In the Game Called Core War Hostile Programs Engage in a Battle of Bits. Scientific American, (250):14-22, 1984.

[76] A. Dorin. Meniscus - Exhibited at the Experimenta House of Tomorrow 5 Sept - 3 Oct 2003, http://www.csse.monash.edu.au/A.d/meniscus.html (accessed June 15th, 2013).

[77] A. Dorin. Plague, Exhibited at Satellite, Australian Pavillion, for Shanghai Bienalle, 3 - 27 Sept 2006, http://www.csse.monash.edu.au/ A.d/plague.html (accessed June 15th, 2013).

[78] A. Dorin. Artificial Life, Death and Epidemics in Evolutionary, Generative Electronic Art. In Rothlauf et al, editor, Proceedings of the 3rd European Workshop on Evolutionary Music and Art, Applications of Evolutionary Computing: EvoWorkshops, pages 448-457. Springer-Verlag, 2005.

[79] A. Dorin. The Sonic Ecosystem. In C. Haines, editor, Proceedings of the Australasian Computer and Music Conference (ACMC), pages 32-37. 2006.

[80] A. Dorin. A Survey of Virtual Ecosystems in Generative Electronic Art. In J. Romero and P. Machado, editors, The Art of Artificial Evolution: A Handbook on Evolutionary Art and Music, pages 289-310. Springer-Verlag, 2007.

[81] A. Dorin. Constellation - Exhibited at Biotope, Cube 37 Gallery, Frankston, Victoria, Australia. Curated by CEMA, 13 July - 9 August, http://www.csse.monash.edu.au/ A.d/constellation.html. 2009.

[82] A. Dorin. Habitat: Engineering in a Simulated Audible Ecosystem. In M. Giacobini, A. Brabazon, S. Cagnoni, G. Caro, A. Ekárt, A.I. Esparcia-Alcázar, M. Farooq, A. Fink, and P. Machado, editors, Applications of Evolutionary Computing, volume 5484 of Lecture Notes in Computer Science, pages 488-497. Springer Berlin Heidelberg, 2009. 
[83] A. Dorin. Pandemic - Generative Software Installation, Exhibited: Bouillants 4, Vernsur-Seiche, Brittany, France, Gaetan Allin and Laurent Dupuis (artistic directors), 22 April - 20 May. 2012.

[84] S. Draves. Evolution and Collective Intelligence of the Electric Sheep. In J. Romero and P. Machado, editors, The Art of Evolutionary Evolution: A Handbook on Evolutionary Art and Music, pages 63-80. Springer-Verlag, Berlin Heidelberg, 2008.

[85] E. Driessens and M. Vestappen. Ima Traveller at Driessens and Vestappen official website - http://notnot.home.xs4all.nl/ima/IMAtraveller.html. 1996.

[86] J. A. Dunne, R. J. Williams, and N. D. Martinez. Food-Web Structure and Network Theory: The Role of Connectance and Size. Proceedings of the National Academy of Sciences of the United States of America, 99:12917-12922, 2002.

[87] G. Dyson. Turing's Cathedral: The Origins of the Digital Universe. Pantheon, 2012.

[88] B. Ebenman and T. Jonsson. Using Community Viability Analysis to Identify Fragile Systems and Keystone Species. Trends in Ecology \& Evolution, 20(10):568-75, October 2005.

[89] U. Eco. The Open Work (Portuguese translation 1989, Difel). Bompiani, 1962.

[90] A. Eldridge and A. Dorin. Filterscape: Energy Recycling in a Creative Ecosystem. In M. Giacobini, editor, Proceedings of EvoWorkshops on Applications of Evolutionary Computing, pages 508-517. Springer-Verlag, 2009.

[91] A. Eldridge, A. Dorin, and J. McCormack. Manipulating Artificial Ecosystems. In M. Giacobini et al, editor, Applications of Evolutionary Computing, volume 4974 of Lecture Notes in Computer Science, pages 392-401. Springer, 2008.

[92] Polson Enterprises. Virtual Pets and Pet Games http://www.virtualpet.com/vp/links/links.htm\#cat (accessed June 15th, 2013).

[93] J. Epstein and R. Axtell. Growing Artificial Societies: Social Science from the Bottom Up. Brookings Institution and MIT Press, Washington, D.C., 1996.

[94] D. B. Fogel. Nils Barricelli - Artificial Life, Coevolution, Self-Adaptation. Computational Intelligence Magazine, IEEE, pages 41 - 45, 2006. 
[95] L. Fogel, A. Owens, and M. Walsh. Artificial Intelligence Through Simulated Evolution. John Wiley \& Sons, 1966.

[96] USC Shoah Foundation Institute for Visual History and Education. Survivors of the Shoah Visual History Foundation, http://college.usc.edu/vhi/aboutus/ (accessed June 15th, 2013), 1994.

[97] S. Forrest and T. Jones. Modeling Complex Adaptive Systems with Echo. In R.J. Stonier and X.H. Yu, editors, Complex Systems: Mechanisms of Adaptation, pages $3-$ 21. IOS Press, 1994.

[98] I. R. Franklin. Evolutionary Change in Small Populations. Conservation Biology: An Evolutionary-Ecological Perspective, pages 135-150, 1980.

[99] Fujitsu. Fin Fin, http://www.virtualpet.com/vp/farm/finfin/finfin.htm (accessed June 15th, 2013).

[100] P. Galanter. Complexism and the Role of Evolutionary Art. In J. Romero and P. Machado, editors, The Art of Evolutionary Evolution: A Handbook on Evolutionary Art and Music, pages 311-334. Springer, 2008.

[101] P. Galanter. The Problem with Evolutionary Art Is .... In Lecture Notes in Computer Science: Applications of Evolutionary Computation, pages 321-330. Springer, 2010.

[102] C. Gloor, P. Stucki, and K. Nagel. Hybrid Techniques for Pedestrian Simulations. In Cellular Automata, volume 3305 of Lecture Notes in Computer Science, pages 581590. Springer, 2004.

[103] S. Grand. Creation: Life and How to Make it. Harvard University Press, 2000.

[104] S. Grand and D. Cliff. Creatures: Entertainment Software Agents with Artificial Life. Autonomous Agents and Multi-Agent Systems, 1(1 / March):39-57, 2004.

[105] G. R. Greenfield. Co-Evolutionary Methods in Evolutionary Art. In J. Romero and P. Machado, editors, The Art of Evolutionary Evolution: A Handbook on Evolutionary Art and Music, pages 357-380. Springer-Verlag, 2008.

[106] G. Guidi, B. Frischer, M. Russo, A. Spinetti, L. Carosso, and L. Micoli. ThreeDimensional Acquisition of Large and Detailed Cultural Heritage Objects. Machine Vision and Applications, 17(6):349-360, 2006. 
[107] S. Hall. Representation: Cultural Representations and Signifying Practices. Sage Publications \& Open University, 1997.

[108] K. Hayles. Narratives of Artificial Life. Routledge, 1996.

[109] K. Hayles. My Mother Was a Computer: Digital Subjects and Literary Texts. The University of Chicago Press, 2005.

[110] D. Helbing. A Fluid Dynamic Model for the Movement of Pedestrians. Complex Systems, 6:391-415, May 1992.

[111] D. Helbing, Moln, I. J. Farkas, and K. Bolay. Self-Organizing Pedestrian Movement. Environment and Planning B: Planning and Design, 28(3):361-383, 2001.

[112] S. Helmreich. Silicon Second Nature: Culturing Artificial Life in a Digital World. University of California Press, 1998.

[113] Jean-Claude Heudin. Lifedrop: A Drop of Life on the Web. In S. Bornhofen, J. Heudin, A. Lioret, and J. Torrel, editors, Virtual Worlds: Artificial Ecosystems and Digital Art Exploration, pages 79-98. Science ebooks, 2012.

[114] Mary Hillier. Automata and Mechanical Toys. Jupiter Books, London, 1976.

[115] C. Hoile. Black Shoals: Evolving Organisms in a World of Financial Data http://www.blackshoals.net/ALife.html (accessed June 15th, 2013). 2012.

[116] J. Holland. Outline for a Logical Theory of Adaptative Systems. Journal of ACM 9, 9(3):297-314, 1962.

[117] J. Holland. Nonlinear Environments Permitting Efficient Adaptation. In Computer and Information Sciences II. Academic Press, 1967.

[118] J.H. Holland. Adaptation. In R. Rosen and F.M. Snell, editors, Progress in Theoretical Biology IV, pages 263-93. Academic Press, 1976.

[119] J.H Holland. Hidden Order: How Adaptation Builds Complexity. Helix Books, 1996.

[120] S. Holtzman. Digital Mantras: The Language of Abstract and Virtual Worlds. MIT Press, 1995.

[121] G. S Hornby and J. B Pollack. Evolving L-Systems to Generate Virtual Creatures. Computers and Graphics, 26(6):1041-1048, 2001. 
[122] G. S. Hornby and J. B. Pollack. The Advantages of Generative Grammatical Encodings for Physical Design. In Evolutionary Computation, pages 600-607. IEEE Press, 2001.

[123] S. Huerre. Agent-Based Crowd Simulation Tool for Theme Park Environments. In 23rd International Conference on Computer Animation and Social Agents (CASA10). Bournemouth University, UK, 2010.

[124] G. Hutzler. The Garden of Chances: A Visual Ecosystem. In Leonardo, volume 33, pages 101-106. MIT Press Journals, 2000.

[125] IBM. Forbidden city - http://www-03.ibm.com/press/us/en/pressrelease/25379.wss (accessed june 15th, 2013), 2009.

[126] M. Iversen. The Aesthetics of Chance. In Margaret Iversen, editor, Chance. Whitechapel Gallery, The MIT Press, 2012.

[127] C. Jacob. Evolutionary and Swarm Design in Science, Art, and Music. In J. Romero and P. Machado, editors, The Art of Artificial Evolution: A Handbook on Evolutionary Art and Music, pages 145-166. Springer-Verlag, 2007.

[128] H. Ji and G. Wakefield. Virtual World-Making in an Interactive Art Installation: Time of Doubles. In S. Bornhofen, J. Heudin, A. Lioret, and J. Torrel, editors, Virtual Worlds: Artificial Ecosystems and Digital Art Exploration. Science ebooks, 2012.

[129] D. Jones. AtomSwarm: A Framework for Swarm Improvisation. In Applications of Evolutionary Computing, pages 423-432. Springer-Verlag, 2008.

[130] E. G. Kim. Josef Albers: Homage to the Square. RM/Fundacion de Arquitectura Tapatia Luis Barragan/ The Josef and Anni Albers Fou, 2009.

[131] L. Klastrup. A Virtual World Aesthetics: Theorising Multi-User Textuality. Internet Research, (3), 2002.

[132] L. Klastrup. A Poetics of Virtual Worlds. In Fifth International Digital Arts and Culture Conference, RMIT, 2003.

[133] T. Kowaliw, J. McCormack, and A. Dorin. An Iteractive Electronic Art System Bsed on Artificial Ecosystemics. In 2011 IEEE Symposium on Artificial Life (ALIFE), pages $162-169$, april 2011. 
[134] J. Koza. Genetic Programming: On the Programming Computers by Means of Natural Selection. MIT Press, 1992.

[135] R. E. Krauss. Under Blue Cup. MIT Press, 2011.

[136] J. Lacan. Le Symbolique, 1 Iimaginaire et le Réel. Bulletin de lÁssociation Freudienne, (1):4-13, 1982.

[137] C. Langton. Artificial Life. In Artificial Life, pages 1-47. Addison-Wesley, 1989.

[138] C. G. Langton, C. Taylor, J. D. Farmer, and S. Rasmussen, editors. Artificial Life II. Addison Wesley, 1992.

[139] W. Latham, S. Shaw, S. Todd, F.F Leymarie, B. Jefferys, and L. Kelley. Using DNA to Generate 3D Organic Art Forms. In M. Giacobini et al., editor, Applications of Evolutionary Computing, LNCS 4974, pages 433-42. Springer, 2008.

[140] B. Latour. Reassembling the Social: An Introduction to Actor-Network-Theory. University Press, 2005.

[141] G. Levin. Golan Levin's website, http://www.flong.com/ (accessed June 15th, 2013). 2013.

[142] P. Lévy. Becoming Virtual: Reality in the Digital Age. Plenum Trade, 1998.

[143] S. Lévy. Artificial Life: A Report From the Frontier Where Computers Meet Biology. First Vintage Books, 1993.

[144] M. Lewis. Evolutionary Visual Art and Design. In J. Romero and P. Machado, editors, The Art of Evolutionary Evolution: A Handbook on Evolutionary Art and Music, pages 3-38. Springer-Verlag, 2008.

[145] A. Lindenmayer. Mathematical Models for Cellular Interactions in Development: Parts i and ii. Journal of Theoretical Biology, (18):280-315, 1968.

[146] A. Lioret. Artificial Life Creation for Cinema. In S. Bornhofen, J. Heudin, A. Lioret, and J. Torrel, editors, Virtual worlds: Artificial Ecosystems and Digital Art Exploration, pages 23-38. Science ebooks, 2012.

[147] Patrick Litchy. Why Art in Virtual Worlds? e-Happenings, Relational Milieux and "Second Sculpture" . volume Automne-Fall. Magazine électronique du CIAC, 2008. 
[148] A.T. Machwe. Towards an Interactive, Generative Design System: Integrating and "Build And Design, Evolve" Approach with Machine Larning for Complex Freeform. In M. Giacobini, editor, EvoWorkshops 2007. LNCS (LNAI, LNBI), vol. 4448, pages 449-458. Springer, 2007.

[149] J. Maïm, S. Haegler, B. Yersin, P. Müller, D. Thalmann, and L. J. V. Gool. Populating Ancient Pompeii with Crowds of Virtual Romans. In Proceedings of the 8th International conference on Virtual Reality, Archaeology and Intelligent Cultural Heritage (VAST'07), pages 109-116, 2007.

[150] L Manovitch. The Language of New Media. The MIT Press, 2001.

[151] L. Margulis, C. Sagan, and D. Sagan. Life, Encyclopaedia Brittanica, online version, http://www.britannica.com/EBchecked/topic/340003/life (accessed June 15th, 2013). 2013.

[152] A. Marr. Gentille curiosité: Wonder-working and the Culture of Automata in the Late Renaissance. In R.J.W. Evans and A. Marr, editors, Curiosity and Wonder from the Renaissance to the Enlightnment. Ashgate, 2006.

[153] C. Martindale. A Neural-Network Theory of Beauty. In C. Martindale, P. Locher, and V.M. Pet- Rov, editors, Evolutionary and Neurocognitive Approaches to Aesthetics, Creativity, and the Arts, pages 181-194. Baywood Publications, 2007.

[154] H. R. Maturana and F. J. Varela. Autopoiesis and Cognition: The Realization of the Living, volume 42 of Boston Studies in the Philosophy of Science. Springer, 1980.

[155] R. M. May. Will a Large Complex System be Stable? Nature, 238:413-414, 1972.

[156] J. McCormack. TURBULENCE an Interactive Installation Exploring Artificial Life. In Visual Proceedings Of ACM Siggraph 94, pages 182-3. Academic Press, 1994.

[157] J. McCormack. Aesthetic, Art, Evolution. In P. Machado, J. McDermott, and A. Carballal, editors, Proceedings of the 2nd Conference on Evolutionary Music and Art (EvoMusart2013), pages 1-12. Centre for Electronic Media Art, 2001.

[158] J. McCormack. Eden: An Evolutionary Sonic Ecosystem. In J. Sosik and P. Kelemen, editors, Lecture Notes in Artificial Intelligence, Vol 2159, Advances in Artificial Life, pages 133-142. Springer-Verlag, 2001. 
[159] J. McCormack. Evolving Sonic Ecosystems. The International Journal of Systems \&' Cybernetics - Kybernetes, 32(1,2):184-202, 2003.

[160] J. McCormack. Aesthetic Evolution of L-Systems Revisited. In G. R. Raid, S. Cagnnoni, J. Branke, D. W. Corne, R. Drechsler, Y. Jin, C. G. Johnson, P. Machado, E. Marchiori, F. Rothlauf, G.D. Smith, and G. Squillero, editors, Proceedings of Applications of Evolutionary Computing (EvoMUSART 2004), volume 3005 of Lecture Notes In Computer Science, pages 477-488. Springer-Verlag, 2004.

[161] J. McCormack. Generative Modelling with Timed L-systems. In J. S. Gero, editor, Proceedings of The First International Conference On Design, Computing And Cognition (DCC’04), pages 157-175. Kluwer Academic Publishers, 2004.

[162] J. McCormack. Open Problems in Evolutionary Music and Art. In F. Rothlauf Al., editor, Applications of Evolutionary Computing, (EvoMUSART 2005), pages 428-436. Springer-Verlag, 2005.

[163] J. McCormack. Creative Ecosystems. In A. Cardoso and G. A. Wiggins, editors, Proceedings of the 4th International Joint Workshop on Computational Creativity, pages pp. 129-136. Goldsmiths, University of London, June 2007.

[164] J. McCormack. Facing the Future: Evolutionary Possibilities for Human-Machine Creativity. In J. Romero and P. Machado, editors, The Art of Evolutionary Evolution: A Handbook on Evolutionary Art and Music, pages 417-451. Springer-Verlag, 2008.

[165] J. McCormack. CodeForm - exhibited at Arts Electronica 2012, http://diotima.infotech.monash.edu.au/-jonmc/sa/artworks/codeform/l (accessed June 15th, 2013). 2012.

[166] J. McCormack and O. Bown. Life's What You Make: Niche Construction and Evolutionary Art. In M. et al. Giacobini, editor, Proceedings of the EvoWorkshops 2009: EvoMUSART, pages 528-537, 2009.

[167] W. McCulloch and W. Pitts. A Logical Calculus of the Ideas Immanent in Nervous Activity. Bulletin of Mathematical Biophysics, 5:115-133, 1943.

[168] L. Meireles. Adeus África, Volto já. Expresso, (2000):70-71, 22011.

[169] E. Mendelowitz. The Emergence Engine: A Behavior Based Agent Development Environment for Artists. In Proceedings of the 12th Conference on Innovative Applications of Artificial Intelligence (IAAI), 2000. 
[170] L. Mignonneau and C. Sommerer. Life Spacies. In ICC Concept Book, pages 96-101. NTT-ICC, Tokyo, 1997.

[171] J. Miller. Simultaneous Statistical Inference. Springer, 1981.

[172] N. Minar, R. Burkhart, C. Langton, and M. Askenazi. The Swarm Simulation System: A Toolkit for Building Multi-Agent Simulations. In Working Paper 96-06-042. Santa Fe Institute, 1996.

[173] J. D. Mitchell and R. E. Lovell. Environment for the Interactive Design of Emergent Art. In ISEA 95: Sixth International Symposium on Electronic Arts, pages 17-21, 1995.

[174] N. Monmarché. Art Made from Artificial Ants. In J. Romero and P. Machado, editors, The Art of Artificial Evolution: A Handbook on Evolutionary Art and Music, pages 227-247. Springer-Verlag, 2007.

[175] G. Monro. The Concept of Emergence in Generative Art. Master of Music, Sydney Conservatorium of Music, 2007.

[176] L. Moura and H. G. Pereira. Man + Robots: Symbiotic Art. Institut d'Art Contemporain, Villeurbanne, 2004.

[177] C. P. H. Mulder, E. Bazeley-White, P. G. Dimitrakopoulos, A. Hector, M. SchererLorenzen, and B. Schmid. Species Evenness and Productivity in Experimental Plant Communities. Oikos, 107:50-63, 2004.

[178] S. R. Musse, C. Babski, T. Capin, and D. Thalmann. Crowd Modelling in Collaborative Virtual Environments. In ACM VRST, pages 115-123. ACM Press, 1998.

[179] K Nagel and M. Schreckenberg. A Cellular Automaton Model for Freeway Traffic. Journal de Physique I, 2(12):2221-2229, December 1992.

[180] C. G Nevill-Manning and I. H. Witten. Compression and Explanation Using Hierarchical Grammars. The Computer Journal, 40(2/3):103-116, 1997.

[181] K. Nishinari, Y. Suma, D. Yanagisawa, A. Tomoeda, A. Kimura, and R. Nishi. Toward Smooth Movement of Crowds. In W. W. F. Klingsch, C. Rogsch, A. Schadschneider, and M. Schreckenberg, editors, Pedestrian and Evacuation Dynamics 2008, pages 293308. Springer, 2010. 
[182] Ovaskainen O. and Meerson B. Stochastic Models of Population Extinction. Trends in Ecology and Evolution, 25(11):643-52, 2010.

[183] N. H. Packard. Intrinsic Adaptation in a Single Model of Evolution. In C. Langton, editor, Artificial Life. Addison-Wesley, 1989.

[184] Y. I. H. Parish and P. Müller. Procedural Modeling of Cities. Proceedings of the 28th Annual Conference on Computer Graphics and Interactive Techniques - SIGGRAPH '01, pages 301-308, 2001.

[185] N. Pelechano, J. M. Allbeck, and N. I. Badler. Controlling Individual Agents in Highdensity Crowd Simulation. In Proceedings of the 2007 ACM SIGGRAPH/Eurographics Symposium on Computer animation, SCA '07, pages 99-108. Eurographics Association, 2007.

[186] N. Pelechano, C. Stocker, J. Allbeck, and N. Badler. Being a Part of the Crowd: Towards Validating VR Crowds Using Presence. In Proceedings of Autonomous Agents and Multiagent Systems (AAMAS), pages 136-142. ACM Press, 2008.

[187] N. Pelechano, C. Stocker, J. Allbeck, and N. Badler. Virtual Crowds: Methods, Simulation, and Control. Synthesis Lectures on Computer Graphics and Animation. Morgan and Claypool Publishers, 2008.

[188] M.D. Petty, F.D. McKenzie, and R.C Gaskins. Requirements, Psychological Models and Design Issues in Crowd Modelling for Military Simulation. In Proceedings of the Huntsville Simulation Conference, 2003.

[189] R. Pfeifer and J. C. Bongard. How the Body Shapes the Way We Think: A New View of Intelligence. MIT Press, 2006.

[190] S. Pimm. Food Webs. The University of Chicago Press, 2002.

[191] E. Pitta. Memorabilia Ultramarina - http://ipsilon.publico.pt/livros/texto.aspx ?id=263210 (accessed March 15, 2012). Público (Ípsilon), 082010.

[192] J. Prophet. Sublime Ecologies and Artistic Endeavors: Artificial Life and Interactivity in the Online Project "TechnoSphere". Leonardo, 29(5):339-344, 1996.

[193] J. Prophet. "Real" Time, "Artificial" Life. Leonardo, 34(4):309-312, 2001. 
[194] J. Prophet and G. Selley. Interview to Gordon Selley and Jane Prophet in Biota 2 Podcast archive, http://www.biota.org/podcast/two.html (accessed June 15th, 2013).

[195] P. Prusinkiewicz and A. Lindenmayer. The Algorithmics Beauty of Plants. SpringerVerlag, 1990.

[196] F. Qiu and X. Hu. Modeling Group Structures in Pedestrian Crowd Simulation. Simulation Modelling Practice and Theory, 18(2):190-205, 2009.

[197] S. F. Railsback and V. Grimm. Agent-Based and Individual-Based Modeling: A Practical Introduction. Princeton University Press, 2011.

[198] S. Ramón y Cajal. Histology of the Nervous System of Man and Vertebrates (History of Neuroscience, No 6) (2 Volume Set). Oxford University Press, 1995.

[199] S. Rasmussen, C. Knudsen, R. Feldberg, and M. Hindsholm. The Coreworld: Emergence and Evolution of Cooperative Structures in a Computational Chemistry. Physica, D(75):1-3, 1990.

[200] S. Raupp Musse and D. Thalmann. Hierarchical Model for Real Time Simulation of Virtual Human Crowds. IEEE Transactions on Visualization and Computer Graphics, 7(2):152-164, 2001.

[201] T. S. Ray. An Approach to the Synthesis of Life. In C. Langton, C. Taylor, J. D. Farmer, and S. Rasmussen, editors, Artificial Life II, Studies in the Sciences of Complexity, volume XI, pages 371-408. Santa Fe Institute, 1991.

[202] T. S. Ray. An Evolutionary Approach to Synthetic Biology: Zen and the Art of Creating Life. In C. G. Langton, editor, Artificial Life, an Overview, pages 179-209. The MIT Press, 1995.

[203] I. Rechenberg. Cybernetic Solution Path of an Experimental Problem. In Royal Aircraft Establishment Translation No. 1122, B. F. Toms, Trans. Ministry of Aviation, Royal Aircraft Establishment, 1965.

[204] K. Reynaldo. Hydroponic herb garden, http://kenrinaldo.com/ (accessed June 15th, 2013), 2011.

[205] C. Reynolds. Big Fast Crowds on PS3. In Proceedings of the 2006 ACM SIGGRAPH Symposium on Videogames, Sandbox '06, pages 113-121. ACM, 2006. 
[206] C. W. Reynolds. Flocks, Herds and Schools: A Distributed Behavioral Model. ACM SIGGRAPH Computer Graphics, 21(4):25-34, 1987.

[207] C. W. Reynolds. Steering Behaviors For Autonomous Characters. In Proceedings of Game Developers Conference, pages 763-782. Miller Freeman Game Group, 1999.

[208] R. Ribeiro. Os retornados estão a abrir o baú http://ipsilon.publico.pt/livros/texto.aspx?id=263209 (accessed March 15, 2012). Público (Ípsilon), 082010.

[209] K. Rinaldo and M. Grossman. The Flock. In Siggraph Visual Proceedings, page 120. ACM, 1993.

[210] J. Romero and P. Machado, editors. The Art of Artificial Evolution: A Handbook on Evolutionary Art and Music. Springer-Verlag, 2007.

[211] R. Rooke. Eons of Genetically Evolved Algorithmic Images. In P. J. Bentley and D. W. Corne, editors, Creative Evolutionary Systems, pages 339-365. Academic Press, 2002.

[212] R. Rucker, R. Sirius, and Q. Mu. Mondo 2000: A User's Guide to the New Edge : Cyberpunk, Virtual Reality, Wetware, Designer Aphrodisiacs, Artificial Life, TechnoErotic Paganism. Perennial, 1992.

[213] T. Saruwatari, Y. Toqunaga, and T. Hoshino. ADIVERSITY: Stepping Up Trophic Levels. In R. A. Brooks and P. Maes, editors, Proceedings of the 4th International Workshop on the Synthesis and Simulation of Living Systems, pages 424-429, 1994.

[214] A. Schadschneider, A. Kirchner, and K. Nishinari. CA Approach to Collective Phenomena in Pedestrian Dynamics. In Proceedings of the 5th International Conference on Cellular Automata for Research and Industry, ACRI '01, pages 239-248. SpringerVerlag, 2002.

[215] M. Schultz and H. Fricke. Managing Passenger Handling at Airport Terminals: Individual-Based Approach for Modeling the Stochastic Passenger Behavior. In Ninth USA/Europe Air Traffic Management Research and Development Seminar (ATM2011), 2011.

[216] M. L. Shaffer. Minimum Population Sizes for Species Conservation. Bioscience, (31):131-134, 1981. 
[217] C. E. Shannon. A Mathematical Theory of Communication. The Bell System Technical Journal, 27:379-423, 1948.

[218] W. Shao and D. Terzopoulus. Populating Reconstructed Archeological Sites with Autonomous Virtual Humans. In J. Gratch, M. Young, R. Aylett, D. Ballin, and O. Patrick, editors, Intelligent Virtual Agents, pages 420-424. Springer, 2006.

[219] E. H. Simpson. Measurement of Diversity. Nature, 163:688, 41949.

[220] K. Sims. Interactive Evolution of Dynamical Systems. In P. Bourgine, editor, Towards a Practice of Autonomous Systems: Proceedings of the First European Conference on Artificial Life, pages 171-178. MIT Press, 1991.

[221] K. Sims. Interactive Evolution of Equations for Procedural Models. The Visual Computer, 9:466-476, 1993.

[222] K. Sims. Evolving 3D Morphology and Behavior by Competition. In R. Brooks and P. Maes, editors, Artificial Life IV: Proceedings of the Fourth International Workshop on the Synthesis and Simulation of Living, pages 28-39. MIT Press, 1994.

[223] K. Sims. Galápagos - http://www.medienkunstnetz.de/works/galapagos/ (accessed June 15th, 2013). In Media Art Net. 1997.

[224] R. V. Solé and J. M. Montoya. Complexity and Fragility in Ecological Networks. Proceedings of the Royal Society B: Biological Sciences / The Royal Society, 268(1480):2039-45, 2001.

[225] C. Sommerer and L. Mignonneau. A-Volve: A Real-time Interactive Environment. In ACM Siggraph Visual Proceedings, pages 172-173, 1994.

[226] C. Sommerer and L. Mignonneau. A-Volve: An Evolutionary Artificial Life Environment. In C. G. Langton and K. Shiomohara, editors, Proceedings of the Fifth Conference on Artificial Life, pages 167-172, 1994.

[227] C. Sommerer and L. Mignonneau. Art as a Living System: Interactive Computer Artworks. System, 32(3):165-173, 1999.

[228] C. Sommerer and L. Mignonneau. Life Spacies II. In Ars Electronica 2000 Next Sex, pages 392-392. Springer Verlag, 2000. 
[229] A. Spinster. Exhibited at Down to Earth, Oxford House Art (Oh! Art), http://nineartists.org/downtoearth/annie work.html (accessed June 15th, 2013). 2007.

[230] K. O. Stanley and R. Miikkulainen. Evolving Neural Networks through Augmenting Topologies. Evolutionary Computation, 10(2):99-127, 2002.

[231] H. Takagi. Interactive Evolutionary Computation: Fusion of the Capabilities of EC Optimization and Human Evaluation. In Proceedings of the IEEE 89(9), pages 12751296, 2001.

[232] T. Taylor and J. Hallam. Studying Evolution with Self-Replicating Computer Programs. In P. Husbands and I. Harvey, editors, Fourth European Conference on Artificial Life, pages 201-204. MIT Press, 1997.

[233] T. J. Taylor. From Artificial Evolution to Artificial Life, PhD Thesis. University of Edinburgh, 1999.

[234] D. Thalmann and S. Musse, Raupp. Crowd Simulation. Springer, 2007.

[235] D. Thalmann, S. R. Musse, and M. Kallmann. Virtual Humans' Behaviour: Individuals, Groups, and Crowds. In Conference on Digital Media Futures, 1999.

[236] O. Théebaud and B. Locatelli. Modelling the Emergence of Resource-Sharing Conventions: an Agent-based Approach. Journal of Artificial Societies and Social Simulation, 4(2), 2001.

[237] S. Todd and W. Latham. Artificial Life or Surreal Art. In P. Bourgine, editor, Towards a Practice of Autonomous Systems: Proceedings of the First European Conference on Artificial Life, pages 504-513. MIT Press, 1991.

[238] S. Todd and W. Latham. Evolutionary Art and Computers. Academic Press, 1992.

[239] M. C. Toyama, A. L. C. Bazzan, and R. da Silva. An Agent-Based Simulation of Pedestrian Dynamics: From Lane Formation to Auditorium Evacuation. In Proceedings of the Fifth International Joint Conference on Autonomous Agents and Multiagent Systems, AAMAS '06, pages 108-110. ACM, 2006.

[240] T. Unemi. SBART2.4: Breeding 2D CG Images and Movies, and Creating a Type of Collage. In The Third International Conference on Knowledge based Intelligent Information Engineering Systems, pages 288-291, 1999. 
[241] Unity3D. Unity3d game engine, www.unity3d.com (accessed June 15th, 2013).

[242] P. Urbano. Playing in the Pheromone Playground: Experiences in Swarm Painting. In F. Rothlauf, J. Branke, S. Cagnoni, D. W. Corne, R. Drechsler, Y. Jin, P. Machado, E. Marchiori, J. Romero, G. D. Smith, and G. Squillero, editors, EvoMusart 2005, pages 527-532, 2005.

[243] P. Urbano. Multi-Agent Coordination and Collective Artificial Paintings. In Proceedings of the International Conference on Agents and Artificial Intelligence (ICAART 2009), pages 19-21, 2009.

[244] J. Ventrella. GenePool: Exploring the Interaction Between Natural Selection and Sexual Selection. In A. Adamatzky and M. Komosinski, editors, Artificial Life Models in Software, pages 81-96. Springer, 2005.

[245] M. Verstappen and E. Driessens. E-Volver - Exhibited at the New Onderzoeksgebouw (research building) of the Leiden University Medical Center, in Amsterdam, http://classic.skor.nl/page/2429/nl?lang=en (accessed June 15th, 2013), 2006.

[246] M. Verstappen and E. Driessens. Driessens and Verstappen at VOUS ETES ICI http://www.vousetesici.nl/artiest/-driessens-verstappen/323 (accessed June 15th, 2013), 2012.

[247] B. Vowk, A. Wait, and C. Schmidt. An Evolutionary Approach Generates Human Competitive Coreware Programs. In M. Bedau, P. Husbands, T. Hutton, S. Kumar, and H. Sizuki, editors, Workshop and Tutorial Proceedings Ninth International Conference on the Simulation and Synthesis of Living Systems(Alife XI), pages 33-36, 2004.

[248] G. Wakefield and H. Ji. Artificial Nature: Immersive World Making. In R. A. Brooks and P. Maes, editors, Applications of Evolutionary Computing LNCS5484, pages 377381. Springer, 2009.

[249] T. Watson and E. Gobeille. Exhibited at DigiPlaySpace, http://www.theowatson.com/site_docs/work.php?id=41 (accessed June 15th, 2013), 2007.

[250] M. Whitelaw. The Abstract Organism: Towards a Prehistory for A-Life Art. Leonardo, 34(No. 4):345-348, 2001.

[251] M. Whitelaw. Metacreation: Art and Artificial Life. MIT Press, 2004. 
[252] N. Wiener. Cybernetics, or Communication and Control in the Animal and the Machine. MIT Press, 1948.

[253] S. W. Wilson. Knowledge Growth in an Artificial Animal. In J. J. Grefenstette, editor, Proceedings of the First International Conference on Genetic Algorithms, pages 16-23. L. Erlbaum Associates Inc., 1985.

[254] S. W. Wilson. Classifier Systems and the Animat Problem. Machine Learning, 2(3/ November):199-228, 1987.

[255] M. Wojtowicz. Exploring Cellular Automata with MCell. In A. Adamatzky and M. Komosinski, editors, Artificial Life Models in Software, pages 233-261. Springer-Verlag, 2005.

[256] S. Wolfram. A New Kind of Science. Wolfram Media Inc, 2002.

[257] L. Yaeger. Computational Genetics, Physiology, Metabolism, Neural Systems, Learning, Vision, and Behavior on Polyworld: Life in a New Context. In C. Langton, editor, Artificial Life III, SFI Studies In The Sciences Of Complexity, Vol. XVII, pages 263-298. Addison-Wesley, 1994.

[258] J. Yuan and D. Gong. Large Population Size IGAs with Individuals' Fitness Not Assigned by User. In Wunsch II D.C. Levine D.S. Jo K. Huang, D., editor, ICIC 2008. LNCS (LNAI), vol. 5227, pages 267-274. Springer, 2008.

[259] Semir Zeki. Prof. Zeki's blog - http://www.neuroesthetics.org/ (accessed June 15th, 2013). 
Glossary 
- ARTIFICIAL LIFE (ALIFE): Sub-field of Artificial Intelligence which focuses on computational systems mimicking some of the processes of natural life and evolution. The term Artificial Life was coined by Christopher Langton and emerged from a conference in simulations of life, in 1987, in the Los Alamos Laboratory. In his words: 'Artificial Life is concerned with tuning the behaviors of such low-level machines that the behavior that emerges at the global level is essentially the same as some behaviour exhibited by a natural living system. [...] Artificial Life is concerned with generating lifelike behavior.'

- ALIFE-ART: Art informed by ALife is known as ALife-art and is discussed in great extent in Whitelaw's work Metacrations.

. Computational Ecosystem: The term computational ecosystem describes an artificial environment populated by a heterogenous population organized in food chains. It is artificial in the sense of being originally man made. This terms distinguishes and contrasts ecosystems built in silico, from biotic artificial ecosystems as seen in traditional Ecology.

. Computational sublime: In the paper from 2001, Art, Emergence and the Computational Sublime, McCormack and Dorin argue the computational sublime is the 'simultaneous feelings of pleasure and fear in the viewer of a process realized in a computing machine'. The overwhelming magnitude/ferocity of nature of the romantic notion of sublime appears here metamorphosed as the overwhelming speed and scale of computers, where operations occur in a dimension which transcends the realm of our direct perceptual experience.

. ECOSYSTEM: In the words of Odum in his book Fundamentals of Ecology, from 1971: 'Any unit that includes all of the organisms ... in a given area interacting with the physical environment so that a flow of energy leads to clearly defined trophic structure, biotic diversity, and material cycles (i.e exchange of materials between living and non living parts) is an ecological system or ecosystem'.

. EMERGENCE: Describes the process of appearance of patterns in complex systems out of a multiplicity of relatively simple interactions. Events in a major level of the system occur as a result of the spontaneous and autonomous interactions at lower scales of detail.

- EVOLUTIONARY ART: Rooted in a generative foundation is a distinct branch of Generative Art. Affiliated with ALife, Evolutionary Art instrumentalizes Evolution and Neo- 
Darwinist precepts in the creation, in silico, of the artistic oeuvre.

. EVOLUTIONARY PROGRAMMING: The EP paradigm, originally conceived by Lawrence J. Fogel in the 1960s, concentrated on models involving a fixed-size population of $\mathrm{N}$ parents, each of which produced a single offspring. The next generation of $\mathrm{N}$ parents was determined by combining both parents and children into a single population of size $2 \mathrm{~N}$. Children are generated asexually by simply creating a copy of the parent and mutating it. EP does not make use of any recombination operator. Child solutions are generated until the population reaches the size of $2 \mathrm{~N}$. A tournament selection ranks the fittest individuals, which are chosen to become the next parents.

. EVOLUTIONARY STRATEGIES: The ES paradigm employs real-coded parameters. In its simplest form, an ES is a simplified and elitist evolutionary algorithm with a population size of 1 . The parent generates a child by mutation of its parameters. If the child is better fitted, then it replaces the parent. In contrast with canonical GAs, which tend to use binary chromosomes, ESs tend to be applied to problems involving strings of real values, and the chromosome is composed of the parameters themselves rather than a binary representation of them.

- FIRST IN FIRST OUT (FIFO): the first element to be inserted in a queue is the first to be removed.

- GENETIC ALGORITHMS: A GA is a model of search algorithms that uses a genetic/evolutionary metaphor. GAs maintain a population of $\mathrm{N}$ individuals consisting of a genotype, a coded version of the parameters and a phenotype, which are the parameters of the solution for the problem. In the canonical GA, the phenotypes of individuals are evaluated and ranked. The score indicates the number of copies each individual will have placed in a list of potential parents. The fitter the individual, the more of his copies exist in this mating pool. Two individuals are then selected randomly from this list to generate a new progeny through random variations in the use of mutation and crossover operators.

. GENETIC PROGRAMming: Popularized by Koza in 1992, GP is a specialized form of GAs where genotypes are usually represented as a variable-sized hierarchical tree-like structures, which can be interpreted as computer programs or functions.

. LAST IN FIRST OUT: The last element to be inserted into a stack is the first to be removed.

. NON PLAYER CHARACTER: A character inhabiting a virtual environment. This has autonomy, agency, and an associated visual representation. For instance, the wolves in World 
of Warcraft that the player needs to interact with in order to complete some particular tasks.

OPEN-ENDED EVOLUTION: Systems where individual components evolve permanently are the holy grail of ALife. This was discussed extensively in McCormack's paper Open Problems in Evolutionary Music and Art [162]. These are systems whose emergent processes redefine the system itself. In contrast with open-systems, one of the requirements of open-ended evolution is the evolution of the processes responsible for unfolding the evolution. Ultimately and ideally, these reach multiple hierarchical levels of emergent organization.

OPEN SYSTEMS: In an evolutionary context, I use this term referring to a system in which individual components continue to evolve continuously, rather than stabilizing when some sort of optimal or stationary state is reached.

TOURNAMENT SELECTION: TS is commonly used in GAs and involves a parameter called the tournament size $t$. When $t=3$ for instance, selection of a single parent is done by randomly choosing three chromosomes from the population and simply taking the fittest of these to be a parent.

. TROPHIC CHAIN: A trophic chain defines the organization or organisms in a food chain. Each trophic level is based on how many links separate the organisms from the primary producers. In trophic levels, there may be one species or a group of species sharing the predators and prey. 
APPENDIX A : 1993:2013 A Survey of Twenty Years of Artistic Use of Computational Ecosystems 


\section{Domain of the survey}

To initiate this study, I scrutinized the proceedings of the main scientific conferences covering this genre of work. These included: Evo*, Generative art, Genetic Evolutionary Computation Conference, the IEEE Congress on Evolutionary Computation, and Artificial Life. I have also looked at a collection of established books with surveys on Artificial Life-art or Evolutionaryart, such as: The Art of Evolutionary Art [210], Metacreations [251], Creative Evolutionary Systems [36] and Virtual Worlds [49]. Finally, I looked at art magazines, such as Art Forum and journals, such as the International Journal of Arts and Technology. From these works, I have selected a sample of [forty] papers. The aim was not to produce an exhaustive scrutiny of the field, but rather to have a significant sample from which we could extrapolate with some confidence. On one hand, this should cover the full spectrum of activity with regards to styles and appropriations of the technique; and on the other hand, it should be distributed throughout the twenty years of the historical time frame. Two selection criteria were followed: the art-criterion and the CE-criterion. The first constrained the selection to works that have been presented or discussed as artistic projects, ideally exhibited in galleries, museums or art festivals or shown/distributed on the internet. The second constrained the selection to instances where the artificial beings that populate the world emulate biological life forms. This includes works where individuals are: represented by Gtype-seeds, or exchange energy or mass, or emulate metabolic cycles (these might include birth, growing morphologies, reproduction and death). Ideally, works should include all these factors, but due to the variety of approaches, this criterion was loosened to the presence of at least one of these factors.

\section{Surveyed works}

1- CodeForm, Jon McCormack, 2012 [165]; 2- SwarmArt, Majid et al., 2012 [10]; 3- Untitled, Bornhofen, 2012 [48]; 4- Where is Lourenço Marques?, Antunes, 2012 [16]; 5- Time of Doubles, Ji \& Wakefield, 2012 [248, 128]; 6- Pandemic, Dorin, 2012 [83]; 7- Vishnu's Dance of Life and Death, Antunes \& Fol Leymarie, 2011 [23]; 8- EvoEco, Kowalik et al., 2011 [133]; 9- Cycles, Bisig \& Unemi, 2010 [42]; 10- Senhora da Graça, Antunes \& Fol Leymarie, 2010 [21]; 11- Constellation, Dorin, 2009 [81]; 12- Habitat, Dorin, 2009 [82]; 13- Untitled experiment, McCormack, 2009 [166]; 14- River Wave, McCormack, 2009 [166]; 15- Fluid Space, Ji \& Wakefield, 2009 [128]; 16- Quorum Sensing, Chen \& Hoyami, 2008 [64]; 17Filterscape, Eldridge et al., 2008 [90]; 18- Infinite Game, Ji \& Wakefield, 2008 [128];19Colour Cycling, Eldridge et al., 2008 [91]; 20- Funky Forest, Watson \& Gobeille, 2007 [249]; 21- xTNZ, Antunes \& Fol Leymarie, 2006 [19]; 22- E-volver, Driessen \& Verstappen, 2006 
[245]; 23- Plague, Dorin, 2006 [77, 78]; 24- Ambient Light, Annie Spinster, 2006 [229]; $25-$ Lifedrop, Heudin, 2004; [113]; 26- Meniscus, Dorin, 2003 [76]; 27- Black Scholes, Hoile, 2001 [74, 115]; 28- Eden, McCormack, 2001 [158]; 29- Biotica, Brown et al., 2001 [55]; 30- Living Melodies, Dahlstedtd \& Nordahl, 2001 [67]; 31- Listening Skies, Berry \& Dorin, 2001 [39]; 32- Iki Iki, Sommerer \& Mignonneau, 2001 [228]; 33- Life Spacies, Sommerer \& Mignonneau, 2000 [228]; 34- Garden of Chances, Hutzler, 2000 [124]; 35- Nerve-Garden, Bruce Damer, 1998 [69]; 36- The Nagual Experiment, Annunziatto, 1998 [14]; 37- Relazioni Emergenti, Annunziatto, 1998 [15]; 38- Technosphere, Prophet, 1996 [192]; 39- EIDEA, Lovell et al., 1995 [173]; 40- A-Volve, Sommerer \& Mignonneau, 1994 [225, 226].

\section{Variables and Taxonomy}

To describe these works, a taxonomy was adapted from Carvalhais' work on Generative art [62]. He suggests, to classify this class of works, an adaptation from Aesperth's taxonomy for cybertexts [6]. The taxonomy includes detailed aspects of the physical implementation of the works in a public exhibition space, while some redundant aspects to the nature of CEs (such as the existence or not of dynamism in the works) are removed. I have divided the variables to consider classification into three groups: (i) to describe contextual properties; (ii) to capture the user experience; and (iii) to describe formal properties.

\section{Contextual variables}

Context: This refers to the main context of the work, to its function. Does the artwork tell or narrate, inform or document, does it visualize, sonify, monitor, mediate, transform, collect or store an event, process or story?

Referentiality: Many works in EvoArt are self-referential or reflect upon life, however a significant number also reflect about societal, political, economic or environmental processes.

Autonomy: This variable describes the focus of the work in terms of input. Is the work independent from external influences (autonomous) or does it need external sources of input, like the meteorological information required by EIDEA (data-driven), or user-input designing and adding new creatures. Accepted values are: autonomous, data-driven, and user. 


\section{User experience (Interactivity)}

We consider now variables describing levels and types of interactivity of a CE.

Perspective: This binary parameter identifies the level of commitment of the audience with respect to the emergence of the work's outcome or storyline. When the audience plays a direct role in the narrative, the work is considered personal, and impersonal otherwise.

User Functions: Members of the audience may observe, explore, activate, control, select, navigate, participate, or leave traces. These interactions are classified as one of three possibilities: interpretative (observe), explorative (explore, navigate, select) or configurative (activate, control, leave trace, participate).

Linking: This variable denotes the existence of devices and processes that make a CE reactive to interactions with the audience. Accepted values are: none, explicit, or implicit. Explicit stands for works where there is a direct involvement of the user, usually via a haptic device, such as a mouse, touch screen, tablet or pod. Implicit is when the body or its physical presence in space is captured with the help of non-interfering devices, such as infra-red cameras.

Modes: Modalities of perception engaging the user are captured by this variable, including the visual, haptic, aural, movement and procedural modes. Movement may include subtle dynamic events, such as finger gestures or eye gaze. Procedural refers to the cognitive dimension of the experience, where in contrast to the other modes, a rational understanding of the processes involved in the construction of the work is implied. For example, Technosphere [192] builds on the illusion of an 'out-there' abstracting the processes involved in its construction, whereas in works such as Cycles [42], this procedural dimension beyond what is seen is emphasized by means of a more abstract form of representation using lines and simple geometric forms in an aesthetic popularized by computer screensavers, which enhances (makes explicit) the presence of the medium and its processes. Modalities of smell and taste could potentially be included here, but this is unnecessary in our study as, to the best of our knowledge, no recent work explores such territories.

Determinability: This binary indicator specifies if different interactions from the audience with the same artefact may result in similar experiences or not. This variable is subordinate to the user-function as unique experiences exist in the explorative and dynamic modes. 
Accepted values are 'yes' or 'no'. Given the subjectivity involved in accessing what are 'similar' experiences, we opted to just classify as determinable those works presented in still a format, as 'drawings' as McCormack puts it [166].

Access: We assume the whole of the artefact is available at all times (e.g. during an exhibit period), but its access can be controlled or random. A controlled situation is illustrated, for example, by Listening Skies [36] where the user creates a 'listener' from whose point of view the world will be perceived, or by Meniscus [76] where the user changes the water level, thus conditioning and controlling the outcomes. A random situation is illustrated by $x T N Z$ [19] where the whole of the virtual environment can be explored in an unconstrained fashion.

Class: This variable is used to indicate the computational class of the work: (1) producing static non-transient outputs; (2) producing static transient outputs; (3) exhibiting complex behaviours. An example of a static non-transient output is a static image. A static transient output defines works that keep changing over time but not in a structural way. Works with complex behaviours are locally structured, partially predictable, and will exhibit random behaviour changes in surprising and unexpected ways.

\section{Formal variables}

The final set of variables is used to describe the formal properties of the artefacts and how they are presented in public.

Format: This describes the physical manifestation of the artefact, including what format was chosen to present a CE to the public. The 'format' can take one of six meanings: (i) installation denotes works designed to transform the perception of space by surrounding (embedding) the user; (ii) sculpture denotes objects that are observed as self-contained arrangements of forms; (iii) video and (iv) interactive-video stand for works where the artefact is presented in a minimalistic technical form with the help of a projector; (v) software-application works are experienced in the intimacy of the computer; (vi) still-imagery stands for printed works of digital photography.

Composition: The second formal variable describes the mode of representation used in the composition, depending on whether if visuals and/or sounds explicitly stand for some external entity and if the work is a collection of representational elements or is abstract. 
Visual Form: This descriptor is used to indicate how individuals are represented visually in the ecosystem. To cover the wide range of approaches, this category accepts a graded scale of values. Individuals can be represented by dots, lines, surfaces, volumes or ephemeral/translucent forms.

Depth: This binary parameter is complementary to the visual form and indicates the presence of foreshortening in the representation. Two values are accepted to denote bi-dimensional (2D or flat) or three-dimensional (3D or volumetric) representations.

Colour: Works may be monochromatic or multi-coloured. Monochromatic works are few, and include black and white as well as grey-level pieces.

SFX: Special effects (SFX) indicates the level of graphical sophistication, such as surface details, texture mixing, or the use of smooth elementary units and solid objects versus complex ones. Accepted values are 'yes' or 'no' (i.e., complex or simple).

Sonification: There are multiple alternatives for the use of sound. The main dichotomy is between pre-recorded and synthesized (in real-time) sounds. A sonification effect can be composed of preselected elements, which might be played for instance as screams by individuals. Alternatively, sounds produced may be granular, i.e. synthesized and played simultaneously by different units of a $\mathrm{CE}$, which is typical of swarming and particle-based approaches.

Display: The CE is ultimately a system running on a computer. This (almost always) requires a visualisation. The technology used to present the $\mathrm{CE}$ to the public is captured by this descriptor: frontal-projection, retro-projection, computer-screen or touch-screen.

Scale: This variable describes the size relationship of the individuals from the virtual population with respect to the human body. Accepted values are: micro for small sizes (typically less than 0.1 meter), human for sizes similar to the human body and parts (up to 3 meters); and macro for other larger sizes (e.g. at architectural/urban scales).

\section{Summary}

A set of variables based on the taxonomy introduced by Carvalhais was presented to classify generative artworks [62]. Some of the original categories were removed - i.e. Dynamics and Transiency - since they are redundant in the context of CEs. Some others had their name 
changed to better clarify their relation to CEs: Individual was changed to Visual Form, Sound to Sonification Blending to SFX, shape to surfaces, transparencies to ephemeral. The nineteen selected variables are as follows:

1- Context (narrate, inform, visualize, sonify, monitor, mediate, transform, collect, store); 2Referentiality (life, societal, political, economic, environmental); 3- Autonomy (autonomous, data-driven, user); 4- Perspective (personal, impersonal); 5- User Functions (interpretative, explorative, configurative); 6- Linking (none, explicit, conditional); 7- Modes (visual, haptic, aural, movement, procedural); 8- Determinability (yes or no); 9- Access (random, controlled); 10- Class (1 (static non-transient), 2 (static transient), 3 (complex)); 11- Format (installation, sculpture, video, interactive-video, sw-app (software-application), still (imagery)); 12- Composition (representational, abstract); 13- Visual Form (dots, lines, surfaces, volumes, ephemeral); 14- Depth (2D, 3D); 15- Colour (mono (chrome), multi (coloured)); 16- SFX (yes (complex), no (simple)); 17- Sonification (pre-selected, granular); 18- Display (frontal (projection), retro (projection), (computer-) screen, or touch); 19- Scale (micro, human, macro)

\section{Results and characterization}

The following tables show the classification for the three main variable types: Contextual, Interactivity, Formal. These tables were produced from a close inspection of: project websites, papers describing the implementations and other material when available (e.g. blogs, reviews). A quick look at the tables shows a great heterogeneity of agendas and outcomes. The content of each table is discussed below, in turn. Note that WisLM [25] and Technosphere [192] appear twice in each table as they have been exhibited both (a) in galleries and (b) on the internet; also, Time of Doubles [248, 128] is a later and enhanced version of Infinite Game [128], and similarly with Pandemic [83] in relation to Plague [77]; note also that tables are organised by date of publication, from most to least recent.

\section{Contextual variables}

First, the contextual aspects of the 40 projects surveyed are considered, as listed in Table 9.1. Summations of variable values are illustrated in Figure 9.1. 


\begin{tabular}{|c|c|c|c|}
\hline WORK & CONTEXT & REFERENTIALITY & Autonomy \\
\hline CODEFORM & VIS+SONIFY & SOCIETAL & USER \\
\hline SWARM-ART & VIS+SONIFY & LIFE & USER \\
\hline BORNHOFEN & VISUALIZE & LIFE & AUTONOMOUS \\
\hline WisLM (A) & VIS+MEDIATE & POLITICAL & AUTONOMOUS \\
\hline WISLM (B) & VIS+MEDIATE & POLITICAL & AUTONOMOUS \\
\hline TIME OF DOUbLeS & VIS+SONIFY & LIFE & USER \\
\hline PANDEMIC & VIS+SONIFY & LIFE & USER \\
\hline VISHNU'S & VISUALIZE & SOCIETAL & AUTONOMOUS \\
\hline EvoEco & VISUALIZE & LIFE & USER \\
\hline Cycles & VISUALIZE & LIFE & USER \\
\hline SraGraca & VISUALIZE & ENVIRONMENTAL & AUTONOMOUS \\
\hline CONSTELlation & VISUALIZE & LIFE & AUTONOMOUS \\
\hline HABITAT & VIS+SONIFY & LIFE & AUTONOMOUS \\
\hline NiCHES & VISUALIZE & LIFE & AUTONOMOUS \\
\hline RIVER WAVE & VIS+SONIFY & LIFE & AUTONOMOUS \\
\hline FLuid SPACE & VIS+SONIFY & LIFE & USER \\
\hline QUORUM SENSING & VIS+SONIFY & LIFE & USER \\
\hline FILTERSCAPE & SONIFY & LIFE & AUTONOMOUS \\
\hline INFINITE GAME & VIS+SONIFY & LIFE & USER \\
\hline Colour Cycling & VISUALIZE & LIFE & AUTONOMOUS \\
\hline FUNKY FOREST & VIS+SONIFY & ENVIRONMENTAL & USER \\
\hline XTNZ & VIS+SONIFY & SOCIETAL & USER \\
\hline E-VOLVER & VISUALIZE & LIFE & USER \\
\hline Plague & VIS+SONIFY & LIFE & USER \\
\hline Ambient Light & VISUALIZE & LIFE & USER \\
\hline LIFEDROP & VISUALIZE & LIFE & AUTONOMOUS \\
\hline
\end{tabular}

Table 9.1: Contextual classification of the 40 surveyed works 


\begin{tabular}{|c|c|c|c|}
\hline WORK & CONTEXT & REFERENTIALITY & AUTONOMY \\
\hline \hline MENISCUS & VIS + SONIFY & LIFE & USER \\
\hline BLACK SCHOLES & VISUALIZE & ECONOMIC & DATA-DRIVEN \\
\hline EDEN & VIS + SONIFY & LIFE & USER \\
\hline BIOTICA & VIS + SONIFY & LIFE & USER \\
\hline LIVING MELODIES & SONIFY & LIFE & AUTONOMOUS \\
\hline LISTENING SKIES & VIS + SONIFY & LIFE & USER \\
\hline IKI IKI & VISUALIZE & LIFE & USER \\
\hline LIFE SPACIES & VISUALIZE & SOCIETAL & USER \\
\hline GARDEN OF CHANCES & VIS + MONITOR & ENVIRONMENTAL & DATA-DRIVEN \\
\hline NERVEGARDEN & VISUALIZE & LIFE & USER \\
\hline NAGUAL EXPERIMENT & VISUALIZE & LIFE & AUTONOMOUS \\
\hline RELAZIONI EMERGENTI & VIS+SONIFY & LIFE & USER \\
\hline TECHNOSPHERE (A) & VISUALIZE & SOCIETAL & USER \\
\hline TECHNOSPHERE (B) & VISUALIZE & SOCIETAL & USER \\
\hline EIDEA & VIS + SON + MONITOR & ENVIRONMENTAL & DATA-DRIVEN \\
\hline A-VOLVE & VISUALIZE & LIFE & USER \\
\hline
\end{tabular}

Table 9.2: Continuation from 9.1
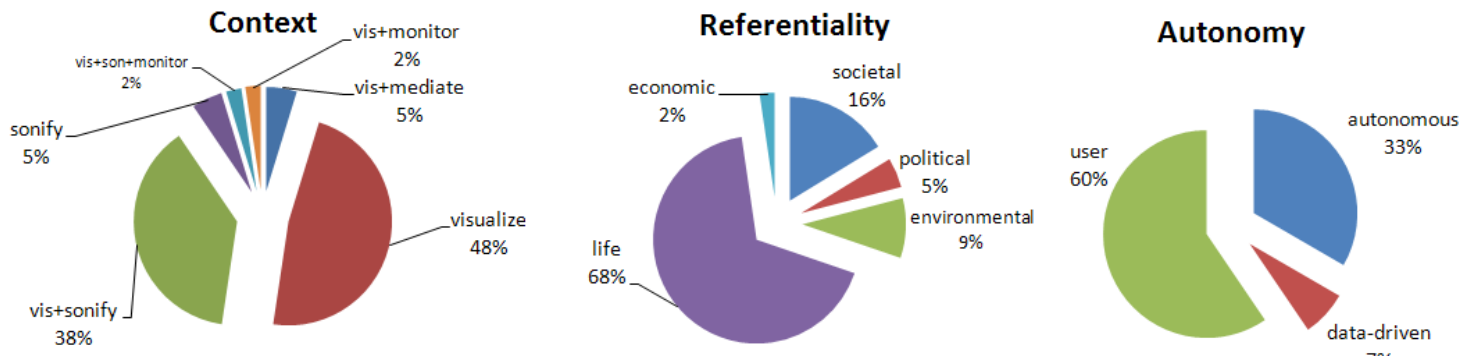

Figure 9.1: Diagrammatic summary of the context of the works.

The first aspect that emerges from the diagrammatic summary (Fig.9.1) is that CEs operate autonomously within an aesthetic that is largely focused around visualizations of processes of life. A close inspection of Table 9.1 reveals that the internal dynamics of the processes of life, such as the spread of diseases in Pandemic [83] or niche-formation in Relazioni Emergenti [14], and self-referentiality, such as the abstract compositions resulting from processes 
of natural selection in Galatema [146], dominate largely representing nearly $70 \%$ of the referentiality spectrum. Together, environmental, societal, political and the economy are themes which represent only about a third of the spectrum. This should not be too surprising if we take into consideration the historical agenda of ALife which has often been used in science to demonstrate biological phenomena and offer suggestions on how such phenomena may arise and function. CEs in particular have been used to draw conclusions about complex adaptive systems. As Whitelaw underlines, ALife art is engaged in the pursuit of an agenda where visualizing and emphasizing life and its processes is a top priority [251]. This situation indicates potential avenues to explore in the future by artists wanting to demarcate themselves from the main themes of previous works.

Looking into the specifics of projects from the point of view of the Context variable, it is not too surprising to find that most works operate in the visual realm (95\%), and almost half of them make use of the aural dimension (45\%). More recent works tend to explore the two modalities integrated together. The other main common denominator is the exploration of interactions with the audience.

Only a small minority or works require external data as input (7\%), but by contrast, the majority requires the audience to be active and perform actions directly impacting the $\mathrm{CE}$ (60\%). Some works are entirely dependent on such actions; for instance, Cycles [42] requires the user to put their hand under the device containing the camera in order to let the virtual agents feed themselves. In other works, however, the user only interferes with the natural evolution of the CE, such as in A-Volve [225, 226], where the audience may insert a new fish in the pool, thus changing the status quo of the virtual tank. The following section analyses the interactive aspect in more detail.

\section{Interactivity}

Table 9.3 and Figure 9.2 capture and summarise the interactivity of the 40 works. 


\begin{tabular}{|c|c|c|c|c|c|c|c|}
\hline WORK & Perspec. & $\begin{array}{c}\text { USER } \\
\text { FUNCTION }\end{array}$ & LINK. & Mode & DET. & ACCESS & Clatss \\
\hline CODEFORM & PERSONAL & CONFIGURATIVE & IMPLICIT & 3 & NO & RANDOM & 2 \\
\hline SWARMIC-ART & PERSONAL & CONFIGURATIVE & EXPLICIT & 2 & NO & RANDOM & 2 \\
\hline BORNHOFEN & IMPERSONAI & INTERPRETATIVE & NONE & 1 & YES & CONTROLLED & 1 \\
\hline WISLM (A) & IMPERSONAI & EXPLORATIVE & EXPLICIT & 3 & NO & RANDOM & 2 \\
\hline WISLM (B) & IMPERSONAI & EXPLORATIVE & EXPLICIT & 3 & NO & RANDOM & 2 \\
\hline Time OF DBL. & PERSONAL & EXPLORATIVE & IMPLICIT & 5 & NO & RANDOM & 3 \\
\hline PANDEMIC & PERSONAL & INTERPRETATIVE & IMPLICIT & 4 & NO & RANDOM & 2 \\
\hline VISHNU'S & IMPERSONAI & INTERPRETATIVH & NONE & 2 & NO & RANDOM & 2 \\
\hline EvoEco & PERSONAL & EXPLORATIVE & EXPLICIT & 3 & NO & CONTROLLED & 1 \\
\hline CyCles & PERSONAL & INTERPRETATIVE & EXPLICIT & 3 & NO & RANDOM & 3 \\
\hline Sra Graca & IMPERSONAI & INTERPRETATIVE & EXPLICIT & 4 & NO & RANDOM & 2 \\
\hline CONSTELlation & IMPERSONAI & INTERPRETATIVH & NONE & 3 & NO & RANDOM & 2 \\
\hline НАВITAT & IMPERSONAI & INTERPRETATIVE & NONE & 3 & NO & RANDOM & 2 \\
\hline NICHES & IMPERSONAI & INTERPRETATIVE & NONE & 2 & YES & CONTROLLED & 1 \\
\hline RiVER WAVE & IMPERSONAI & INTERPRETATIVE & NONE & 2 & NO & RANDOM & 2 \\
\hline Fluid SPACE & PERSONAL & EXPLORATIVE & EXPLICIT & 5 & NO & RANDOM & 3 \\
\hline QuORuM SENS. & PERSONAL & EXPLORATIVE & IMPLICIT & 4 & NO & RANDOM & 3 \\
\hline FILTERSCAPE & IMPERSONAI & INTERPRETATIVE & NONE & 2 & NO & RANDOM & 2 \\
\hline INFINITE GAME & PERSONAL & EXPLORATIVE & EXPLICIT & 5 & NO & RANDOM & 3 \\
\hline Colour CyCling & IMPERSONAI & INTERPRETATIVE & NONE & 2 & NO & RANDOM & 1 \\
\hline
\end{tabular}

Table 9.3: The user (interactivity) functions of the 40 surveyed works. 


\begin{tabular}{|c|c|c|c|c|c|c|c|}
\hline WORK & Perspec. & $\begin{array}{c}\text { USER } \\
\text { FUNCTION }\end{array}$ & LINK. & MODE & DET. & ACCESS & Class \\
\hline FUNKY FOREST & PERSONAL & EXPLORATIVE & IMPLICIT & 5 & NO & RANDOM & 3 \\
\hline XTNZ & PERSONAL & EXPLORATIVE & EXPLICIT & 3 & NO & RANDOM & 2 \\
\hline E-VOLVER & PERSONAL & EXPLORATIVE & EXPLICIT & 3 & NO & CONTROLLED & 1 \\
\hline Plague & PERSONAL & EXPLORATIVE & IMPLICIT & 3 & NO & RANDOM & 2 \\
\hline Ambient Light & PERSONAL & INTERPRETATIVE & EXPLICIT & 2 & NO & RANDOM & 2 \\
\hline LIFEDROP & IMPERSONAI & CONFIGURATIVE & NONE & 2 & NO & RANDOM & 2 \\
\hline Meniscus & PERSONAL & CONFIGURATIVE & EXPLICIT & 5 & NO & RANDOM & 2 \\
\hline BLACK SQ. & IMPERSONAI & INTERPRETATIVE & NONE & 4 & NO & RANDOM & 2 \\
\hline EDEN & PERSONAL & EXPLORATIVE & IMPLICIT & 4 & NO & RANDOM & 2 \\
\hline BIOTICA & PERSONAL & EXPLORATIVE & EXPLICIT & 3 & NO & RANDOM & 2 \\
\hline Living Melod. & IMPERSONAI & INTERPRETATIVE & NONE & 1 & NO & RANDOM & 2 \\
\hline LISTEN. SKIES & PERSONAL & EXPLORATIVE & EXPLICIT & 4 & NO & RANDOM & 2 \\
\hline IKI IKI & PERSONAL & CONFIGURATIVE & EXPLICIT & 3 & NO & RANDOM & 3 \\
\hline LIFE SPACIES & PERSONAL & CONFIGURATIVE & IMPLICIT & 5 & NO & RANDOM & 2 \\
\hline GARDEN OF CH. & IMPERSONAI & INTERPRETATIVE & NONE & 2 & NO & RANDOM & 2 \\
\hline NERVEGARDEN & PERSONAL & EXPLORATIVE & EXPLICIT & 2 & NO & RANDOM & 2 \\
\hline NAGUAL EXP. & IMPERSONAI & INTERPRETATIVE & NONE & 1 & YES & CONTROLLED & 1 \\
\hline RELAZIONI EMER. & PERSONAL & CONFIGURATIVE & IMPLICIT & 3 & NO & RANDOM & 3 \\
\hline TEChnos. (A) & PERSONAL & CONFIGURATIVE & EXPLICIT & 2 & NO & CONTROLLED & 2 \\
\hline TEChNOS. (B) & PERSONAL & CONFIGURATIVE & EXPLICIT & 2 & NO & CONTROLLED & 2 \\
\hline EIDEA & IMPERSONAI & INTERPRETATIVE & NONE & 3 & NO & RANDOM & 3 \\
\hline A-VOLVE & PERSONAL & EXPLORATIVE & EXPLICIT & 4 & NO & RANDOM & 2 \\
\hline
\end{tabular}

Table 9.4: Continuation from 9.3 


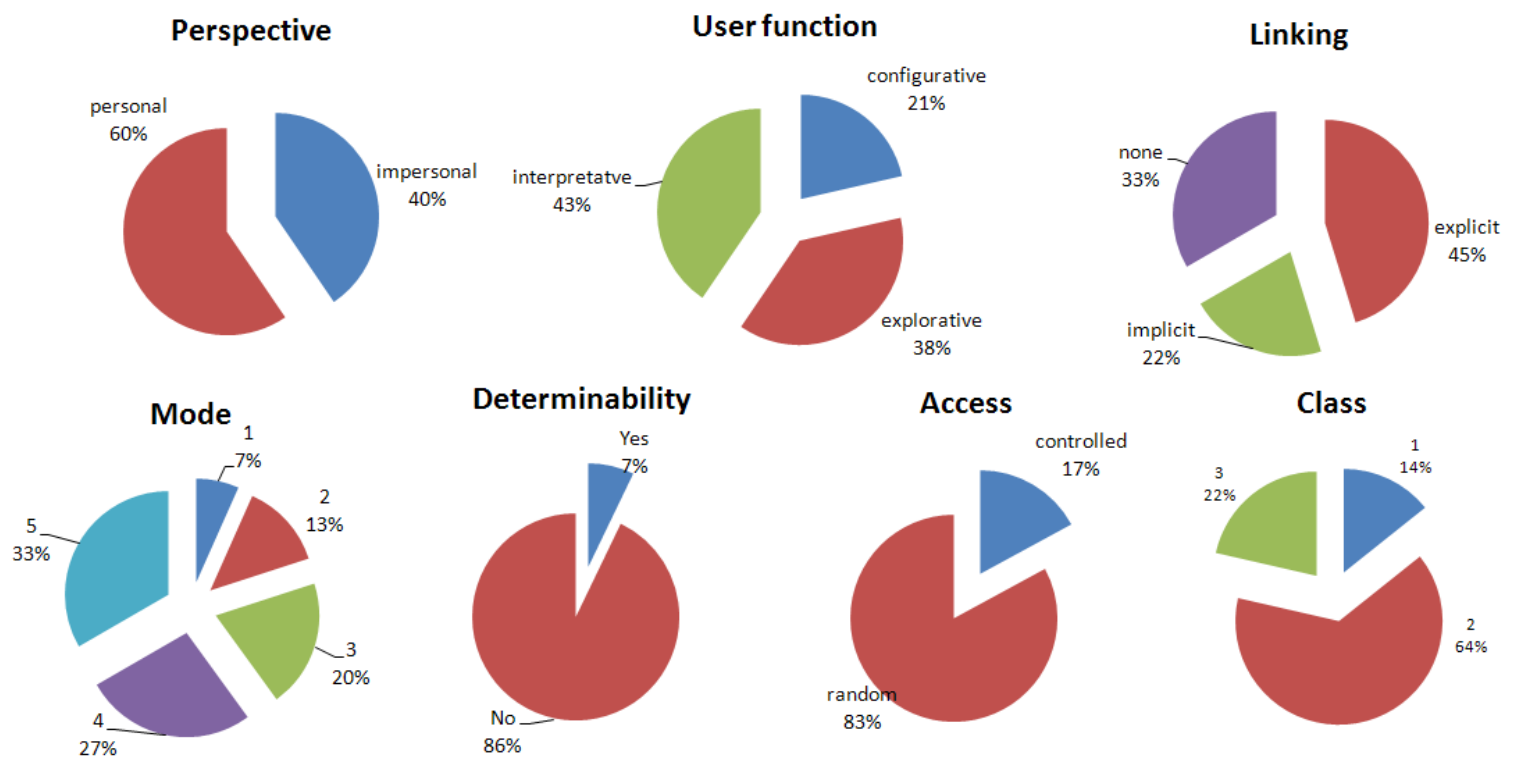

Figure 9.2: Diagrammatic summary of the Interactivity of the works.

With no exception, all the works under scrutiny produce either a visual or audio outcome to be experienced and appreciated. This naturally results from the selection criteria used, which required works to be artistic or exhibited in public. The audience is an integral part of most works and the interactive devices are explicit and visible for the large part (45\%). In $22 \%$ of the instances however, the body presence is captured without the help of any accessory haptic devices, usually by means of computer vision techniques. This percentage would have substantially risen if only interactive works were considered. Also, note that in the near future, the new possibilities provided by interactive technologies, such as Microsoft's Kinect and its descendants, are likely to have a major impact on the field (i.e. raise the influence of body movement and gesture-based interactions).

The explorative component is present in $38 \%$ of the works, but only a rather small number of projects $(21 \%)$ let the user configure the settings (or interfere with the evolution). Meniscus [76] provides an example where the audience controls the level of virtual water in the simulation. This small percentage of works allowing configurative tasks is rather surprising, in particular since only the actions of adding or removing members of the population (of the $\mathrm{CE}$ ) were considered as part of this category. As mentioned earlier, one conclusion to derive from our study is the untapped potential for greater levels of interactivity, in particular for the exploration of the configurative roles played by the audience. 
It must be underlined that although a $\mathrm{CE}$ is in essence a complex system often exhibiting non-determinable outcomes, it remains constrained by parameters restricted to operate only within set ranges ${ }^{1}$. For instance, if the programmer designs the system as composed by individuals represented by triangles, these will never become circles or take other geometrical forms. Having full access to non-determinability remains a 'holy grail' of ALife: i.e., producing some open-ended systems which automatically generate and change their own rules of production.

In terms of the Linking variable, we can notice that nearly half the works use explicit interaction devices, such as a mouse in $x T N Z$, hands blocking a sensor's view in Cycles, or wearing special goggles as in Biotica [55]. For nearly another quarter of the works, the presence of the user is captured in a more discrete, implicit way (22\%). In terms of the Class variable, the majority of works (64\%) keep changing over time but not in a structural way (class \#2), while a significant number exhibit more complex behaviours (class \#3 at 22\%). As for the Mode variable, about $2 / 3$ of the works explore simultaneously four or more of the properties analysed: visual, haptic, aural, movement from the user and perception of procedural qualities. In terms of the Perspective variable, for $60 \%$ of the cases the user has a personal engagement with the story, either by creating a new creature (e.g. in AVolve [225]), or adding food/energy (e.g. in Fluid Space [128]), or introducing a disease to the virtual world (e.g. in Pandemic [83]).

Finally, the Access variable provides us with a clear pattern that distinguishes CEs from other interactive media instances, such as games, as a large majority (83\%) of the works represented here do not offer 'levels' or hidden areas of the world that the user can activate by means of their actions.

\section{Formal parameters}

Table 9.5 presents the classification of the 40 works with regards to their formal variables making explicit their mode of presentation or exhibition.

\footnotetext{
${ }^{1}$ Note that most works $(86 \%)$ are not determinable, the only exceptions being works presented to the public as static pictures or 'drawings'.
} 


\begin{tabular}{|c|c|c|c|c|c|c|c|c|c|}
\hline WORK & FORMAT & Сомр. & VISUAL & DEPTH & COLOR & SFX & Sonific. & DisPlay & SCALE \\
\hline CODEFORM & VIDEO & REPRES & VOLUMES & $3 \mathrm{D}$ & MULTI & YES & GRANULAR & $\begin{array}{l}\text { FRONTAL- } \\
\text { PROJ }\end{array}$ & HUMAN \\
\hline SWARMIC-ART & WEBSITE & REPRES & DOTS & $2 \mathrm{D}$ & $\mathrm{B} / \mathrm{W}$ & NO & GRANULAR & $\begin{array}{l}\text { COMP- } \\
\text { SCREEN }\end{array}$ & MICRO \\
\hline BORNHOFEN & STILL & ABSTRACT & LINES & $2 \mathrm{D}$ & $\mathrm{B} / \mathrm{W}$ & NO & NONE & NA & MICRO \\
\hline WISLM (A) & VIDEO-INTER & REPRES & VOLUMES & $3 \mathrm{D}$ & MULTI & NO & PRESELECT & $\begin{array}{c}\text { FRONTAL- } \\
\text { PROJ }\end{array}$ & MICRO \\
\hline WISLM (B) & WEBSITE & REPRES & VOLUMES & $3 \mathrm{D}$ & MULTI & NO & PRESELECT & $\begin{array}{l}\text { COMP- } \\
\text { SCREEN }\end{array}$ & MICRO \\
\hline TIME OF DBL & INSTALLATION & ABSTRACT & VOL+EPHE & $3 \mathrm{D}$ & MULTI & YES & GRANULAR & RETRO-PROJ & HUMAN \\
\hline Pandemic & INSTALLATION & ABSTRACT & SURFACES & $3 \mathrm{D}$ & MULTI & NO & GRANULAR & $\begin{array}{c}\text { FRONTAL- } \\
\text { PROJ }\end{array}$ & HUMAN \\
\hline VISHNU'S & WEBSITE & REPRES & VOLUMES & $3 \mathrm{D}$ & MULTI & NO & PRESELECT & $\begin{array}{l}\text { COMP- } \\
\text { SCREEN }\end{array}$ & MICRO \\
\hline EvoEco & WEBSITE & ABSTRACT & DOTS & $2 \mathrm{D}$ & MULTI & NO & NONE & $\begin{array}{l}\text { COMP- } \\
\text { SCREEN }\end{array}$ & MICRO \\
\hline CyCles & SCULPTURE & ABSTRACT & LINES+SURF & $2 \mathrm{D}$ & MULTI & YES & NONE & $\begin{array}{c}\text { VERTICAL- } \\
\text { PROJ }\end{array}$ & MICRO \\
\hline Sra Graca & SW-APP & ABSTRACT & VOL+EPHE & $3 \mathrm{D}$ & MULTI & YES & NONE & $\begin{array}{l}\text { COMP- } \\
\text { SCREEN }\end{array}$ & MICRO \\
\hline CONSTEllation & VIDEO & REPRES & SURFACES & $2 \mathrm{D}$ & MULTI & YES & No & RETRO-PROJ & MACRO \\
\hline $\begin{array}{l}\text { HABITAT } \\
\end{array}$ & SW-APP & REPRES & SURFACES & $2 \mathrm{D}$ & MULTI & NO & PRESELECT & $\begin{array}{l}\text { COMP- } \\
\text { SCREEN }\end{array}$ & MICRO \\
\hline NICHES & STILL & ABSTRACT & LINES & $2 \mathrm{D}$ & MONO & NO & NONE & NA & MICRO \\
\hline River WaVe & SW-APP & ABSTRACT & NA & NA & NA & NA & GRANULAR & NA & NA \\
\hline Fluid SPACE & INSTALLATION & ABSTRACT & VOL+EPHE & $3 \mathrm{D}$ & MULTI & YES & GRANULAR & $\begin{array}{c}\text { FRONTAL- } \\
\text { PROJ }\end{array}$ & HUMAN \\
\hline QUORUM SENS. & INSTALLATION & ABSTRACT & VOL+EPHE & $3 \mathrm{D}$ & MULTI & YES & NA & $\begin{array}{c}\text { VERTICAL- } \\
\text { PROJ }\end{array}$ & HUMAN \\
\hline FILTERSCAPE & SW-APP & ABSTRACT & NA & NA & NA & NA & GRANULAR & NA & HUMAN \\
\hline INFINITE GAME & INSTALLATION & ABSTRACT & VOL+EPHE & $3 \mathrm{D}$ & MULTI & YES & GRANULAR & $\begin{array}{c}\text { FRONTAL- } \\
\text { PROJ }\end{array}$ & HUMAN \\
\hline Colour Cycling & VIDEO & ABSTRACT & DOTS & $2 \mathrm{D}$ & MULTI & NO & NA & $\begin{array}{l}\text { COMP- } \\
\text { SCREEN }\end{array}$ & MICRO \\
\hline FUNKY FOREST & INSTALLATION & REPRES & SURFACES & $2 \mathrm{D}$ & MULTI & NO & PRESELECT & $\begin{array}{c}\text { FRONTAL- } \\
\text { PROJ/ } \\
\text { VERTICAL- } \\
\text { PROJ }\end{array}$ & MICRO \\
\hline XTNZ & VIDEO-INTER & ABSTRACT & VOL+EPHE & $3 \mathrm{D}$ & MULTI & YES & PRESELECT & $\begin{array}{c}\text { FRONTAL- } \\
\text { PROJ }\end{array}$ & MICRO \\
\hline E-VOLVER & VIDEO-INTER & ABSTRACT & DOTS & $2 \mathrm{D}$ & MULTI & NO & NA & FLAT-PANEL & MICRO \\
\hline Plague & INSTALLATION & ABSTRACT & SURFACES & $3 \mathrm{D}$ & MULTI & NO & GRANULAR & $\begin{array}{c}\text { FRONTAL- } \\
\text { PROJ }\end{array}$ & HUMAN \\
\hline
\end{tabular}

Table 9.5: The formal classification of the 40 surveyed works. 


\begin{tabular}{|c|c|c|c|c|c|c|c|c|c|}
\hline WORK & FORMAT & Сомр. & VISUAL & DEPTH & Color & SFX & Sonific. & DISPLAY & SCALE \\
\hline Ambient Light & INSTALLATION & ABSTRACT & SURFACES & $2 \mathrm{D}$ & MULTI & NO & NA & FLAT-PANEL & MICRO \\
\hline LIFEDROP & WEBSITE & REPRES & LINES & $2 \mathrm{D}$ & MULTI & NO & NA & COMP-SCREEN & MICRO \\
\hline MENISCUS & VIDEO-INTER & ABSTRACT & SURFACES & $2 \mathrm{D}$ & MULTI & NO & NA & FLAT-PANEL & MICRO \\
\hline BLKSCHOLES & INSTALLATION & ABSTRACT & DOTS & $2 \mathrm{D}$ & MULTI & NO & NA & RETRO-PROJ & HUMAN \\
\hline EDEN & INSTALLATION & ABSTRACT & SURFACES & $2 \mathrm{D}$ & MULTI & $\mathrm{NO}$ & GRANULAR & RETRO-PROJ & HUMAN \\
\hline BIOTICA & SCULPTURE & ABSTRACT & VOLUMES & $3 \mathrm{D}$ & MULTI & NO & GRANULAR & RETRO-PROJ & HUMAN \\
\hline LIVINGMELODIES & SW-APP & ABSTRACT & NA & NA & NA & NA & GRANULAR & NA & NA \\
\hline LISTENINGSKIES & VIDEO-INTER & REPREST & SURFACES & $3 \mathrm{D}$ & MULTI & NO & GRANULAR & FRONTAL-PROJ & HUMAN \\
\hline IKI IKI & MOBILE & ABSTRACT & SURFACES & $2 \mathrm{D}$ & MULTI & NO & NA & MOBILE-PHONE & MICRO \\
\hline GARDEN OF CH. & SW-APP & ABSTRACT & SURFACES & $2 \mathrm{D}$ & MULTI & NO & $\mathrm{NA}$ & COMP-SCREEN & MICRO \\
\hline NERVEGARDEN & WEBSITE & REPRES & VOLUMES & $3 \mathrm{D}$ & MULTI & NO & NA & COMP-SCREEN & MICRO \\
\hline NAGUAL EXP. & STILL & ABSTRACT & LINES & $2 \mathrm{D}$ & $\mathrm{B} / \mathrm{W}$ & NO & NA & NA & MICRO \\
\hline RELAZIONi EMERG & VIDEO-INTER & ABSTRACT & LINES & $2 \mathrm{D}$ & MULTI & NO & GRANULAR & RETRO-PROJ & HUMAN \\
\hline TEChNOSPH. (A) & VIDEO-INTER & REPRES & VOLUMES & $3 \mathrm{D}$ & MULTI & NO & NA & FRONTAL-PROJ & HUMAN \\
\hline TECHNOSPH. (B) & WEBSITE & REPRES & VOLUMES & $3 \mathrm{D}$ & MULTI & NO & NA & COMP-SCREEN & MICRO \\
\hline EIDEA & VIDEO & REPRES & VOLUMES & $3 \mathrm{D}$ & MULTI & YES & GRANULAR & FRONTAL-PROJ & HUMAN \\
\hline A-Volve & SCULPTURE & REPRES & VOLUMES & $3 \mathrm{D}$ & MULTI & NO & NA & FLAT-PANEL & HUMAN \\
\hline
\end{tabular}

Table 9.6: Continuation from Table 9.5 

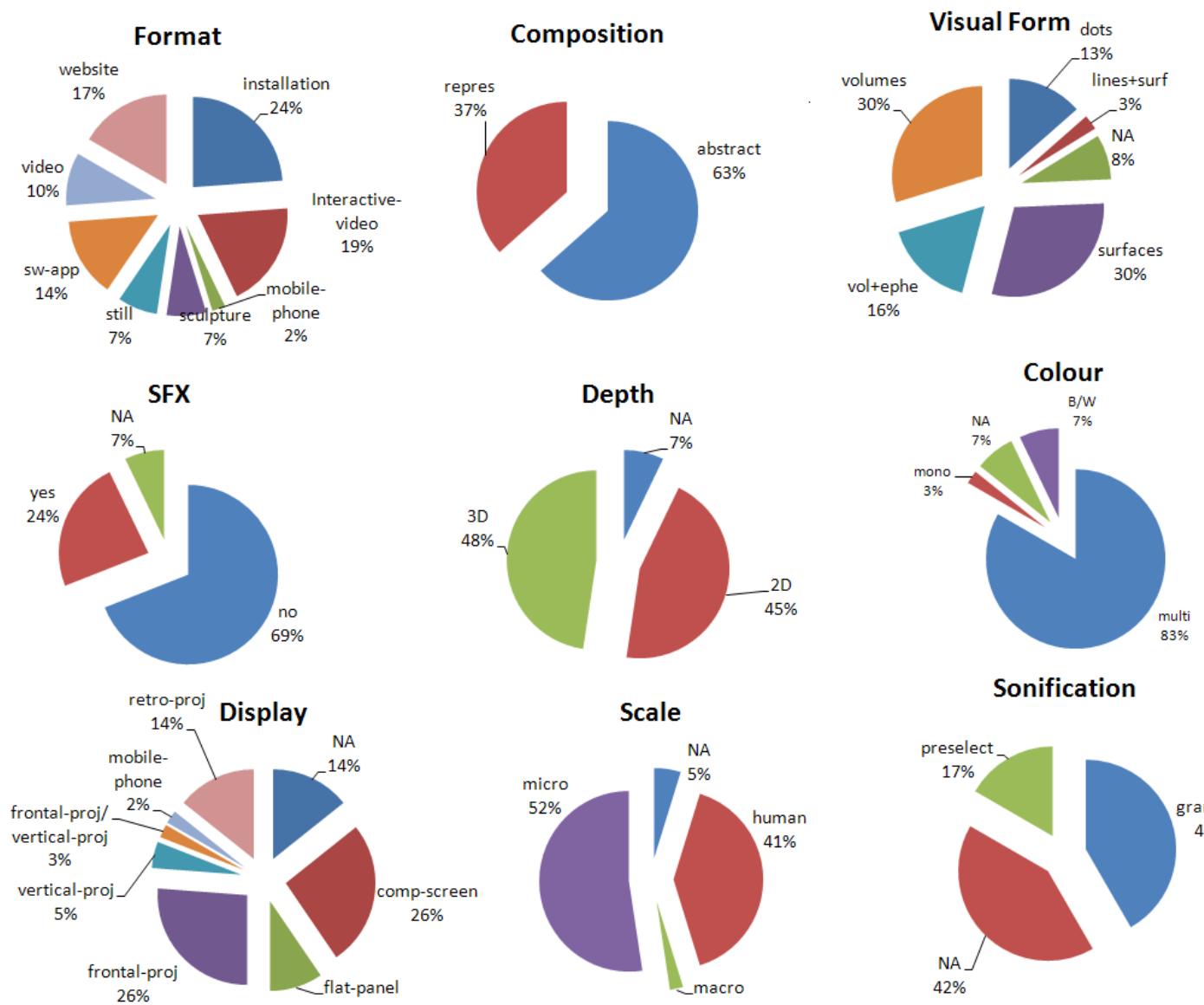

Sonification

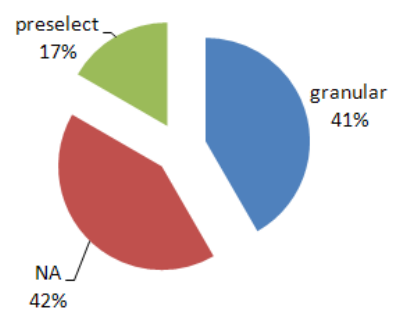

Figure 9.3: Diagrammatic summary of the formal presentation.

The openness and plasticity of CEs is demonstrated by this study. In the works analysed, while there is a similarity of methods used, this is combined with a great disparity of outcomes and heterogeneity in the Formats of production. The personal computer is not the privileged mode of operation, with only one third of the works taking the format of websites or software applications. Works exhibited in gallery spaces dominate the sample (nearly 70\%). From this large group, video projections and interactive-video clearly dominate. As a consequence, works tend to operate at human body (41\%) or smaller (micro) scales (52\%), and only one project exploits macro scales (Constellation [81]).

When it comes to the Composition, the representational scheme of choice composed of the shape, colour, the trace used, and the scale of the artefacts was considered. Results suggest that there is a clear dominance of the abstract over the representational. This is not too surprising taking into consideration the agenda from ALife of 'life as it could be'. In terms of Visual 
Forms, dots and lines are rarely used in comparison to surface shapes and volumes. Dots usually produce plasma-like looking works as a function of changing CE dynamics, whereas surface shapes and volumes are the carriers of more traditional modes of representation, including the use of perspective and foreshortening.

As could be expected, the Colour variable is dominated by multi-chromatic works over black and white or monochrome works. Surprisingly however, there is not much sophistication involved in the resulting visualizations. Authors seem to prefer solid forms rather than SFX, such as blending textures or using complex graphics. This result might be somewhat biased due to the temporal scale of the particular sample under analysis, which includes a fair number of works from the 1990's and early 2000's when rendering sophisticated visuals in real time was comparatively much harder than in recent years.

When it comes to the Depth variable, there is no clear dominance of the use of 3D versus $2 \mathrm{D}$. This might change in the future, as $3 \mathrm{D}$ technologies (of production and display) become more accessible.

The freedom of expression of CEs is again suggested when the Sonification variable is considered. Granular and synthesized sounds are used in $41 \%$ of the works, while only $17 \%$ use pre-selected more "naturalistic" sounds. Examples of sonification include the literal translation of CE dynamics (e.g. Time of Doubles [128]), abstract formulations (e.g. Living Melodies [67]), or having visuals being entirely secondary while the focus of the work is on the sound generated (e.g. Filterscape [90]). Surprising is the fact that there is no sonification at all in a large number of the works (42\%), as reported in associated papers, blogs or websites. It is also worth mentioning that some works use sounds independently from the CE's dynamics (such as in WisLM [25] and Vishnu's [23]). 


\section{APPENDIX B: Auxiliary Data for the Comparative Study on Algorithms for Persistence}




\section{Summary of the trials}

Pattern Defines the type of behavioural pattern of the community. This can have one of two values: a) EXT or b) OVR, meaning respectively, communities with a strong intrinsic pressure towards extinction, and populations with strong intrinsic pressure towards overpopulation;

Size This variable indicates the average size of the population;

Forced actions This is the central variable under study since it depicts the number of interventions that were required to maintain the system out of collapse. It is the sum of Artificial insertions, Artificial removals, Inhibited Insertions and Inhibited Removals;

Artificial insertions This is a numeric variable that captures the dimension of extra individuals that were inserted by the system in the community in order to keep it 'alive'. These are individuals that appear in the community out of the natural evolutionary course of the simulation, and as a result from the mitigation strategies;

Artificial removals This indicator shows the number of individuals that have been removed arbitrarily out of their natural life cycle due to a carrying capacity being reached;

Inhibited Insertions Number of insertions resulting from the interaction of the individuals in the community that have been inhibited by the system to avoid situations of overpopulation;

Inhibited Removals Number of times that individuals have been kept unnaturally alive to avoid an extinction. 


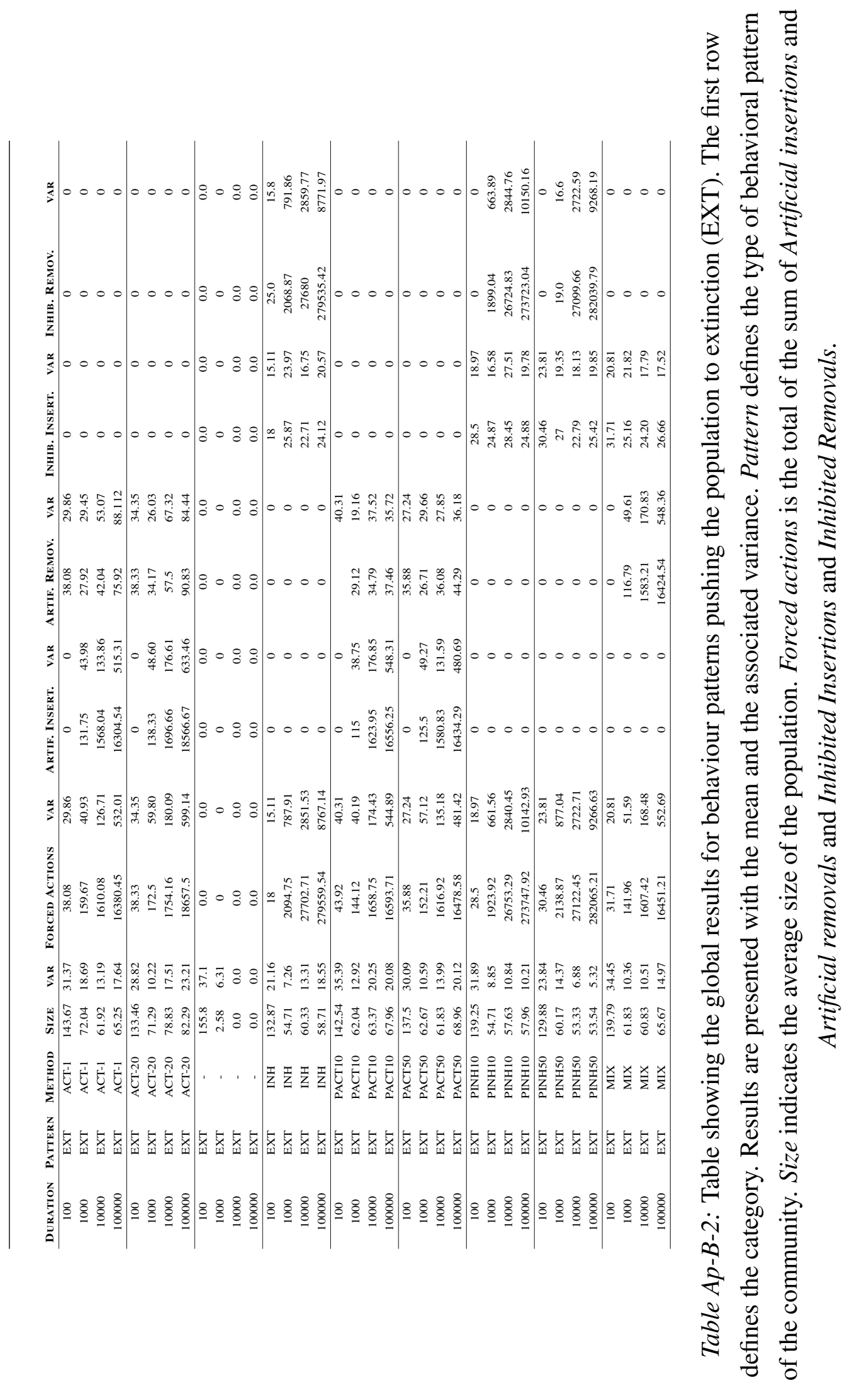



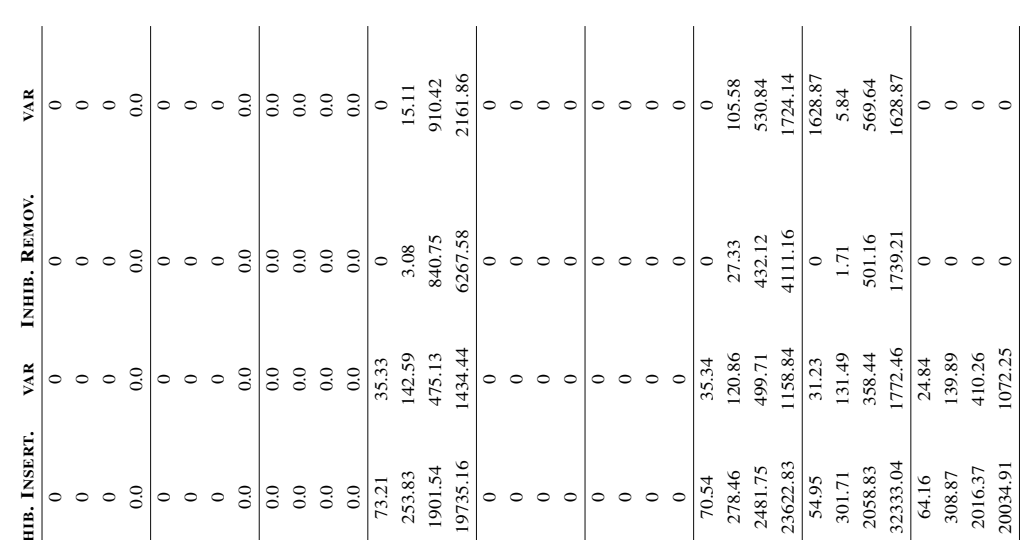

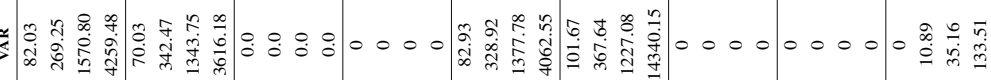

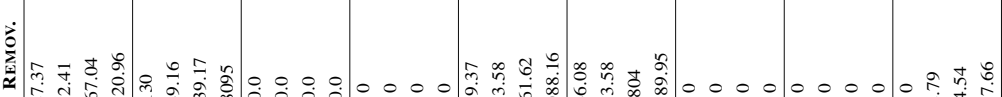

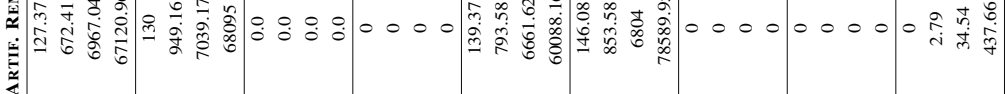

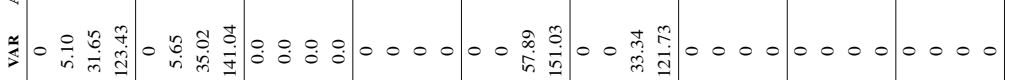

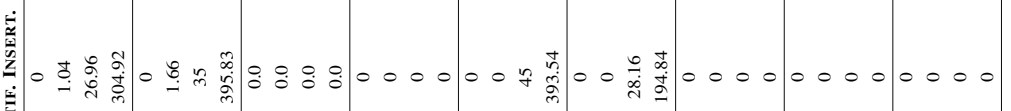

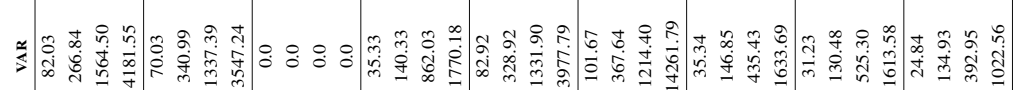

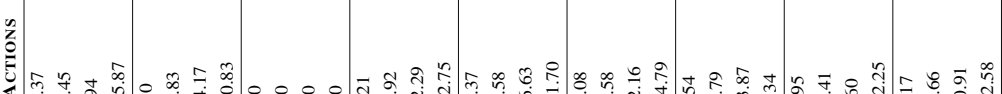

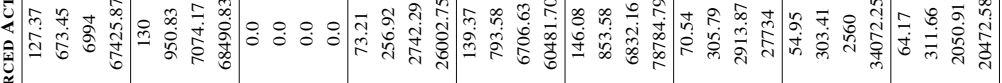

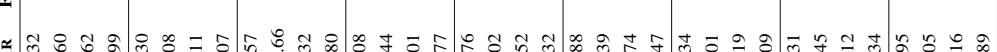

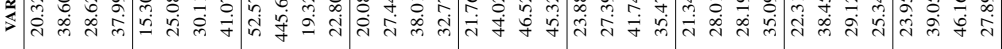

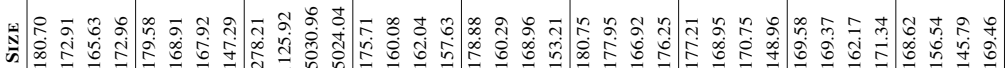

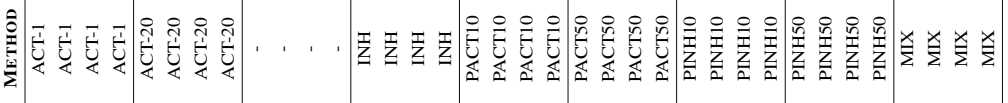

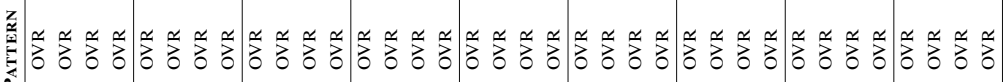

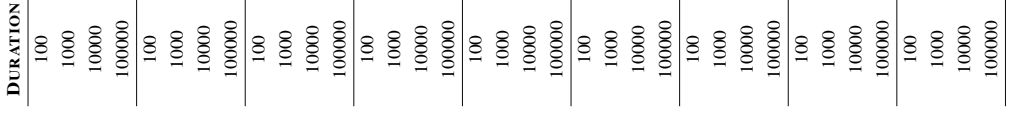

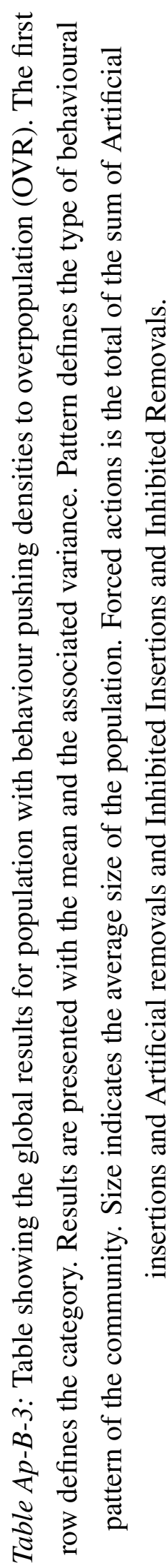




\section{Comparison between the Mixed strategy and the other strategies.}

\begin{tabular}{|c|c|c|c|c|}
\hline & \multicolumn{4}{|c|}{100 OVR } \\
\hline & DIFF & $\begin{array}{l}\text { LWR } \\
\text { UPR }\end{array}$ & $P$ & RES \\
\hline \multirow{2}{*}{$\begin{array}{l}\text { MIX - } \\
\text { ACT1 }\end{array}$} & \multirow{2}{*}{-68.37} & -113.22 & \multirow{2}{*}{0.01} & \multirow{2}{*}{$\mathrm{X}-\mathrm{O}$} \\
\hline & & -23.52 & & \\
\hline \multirow{2}{*}{$\begin{array}{l}\text { MIX - } \\
\text { ACT20 }\end{array}$} & \multirow{2}{*}{-57.20} & $-102 / 06$ & \multirow{2}{*}{0.01} & \multirow{2}{*}{$\mathrm{X}-\mathrm{O}$} \\
\hline & & -12.35 & & \\
\hline \multirow{2}{*}{$\begin{array}{l}\text { MIX - } \\
\text { INH }\end{array}$} & \multirow{2}{*}{4.87} & -39.97 & \multirow{2}{*}{0.99} & \multirow{2}{*}{-} \\
\hline & & 49.72 & & \\
\hline \multirow{2}{*}{$\begin{array}{l}\text { PACT1 - } \\
\text { MIX }\end{array}$} & \multirow{2}{*}{66.41} & 21.56 & \multirow{2}{*}{0.001} & \multirow{2}{*}{ o-X } \\
\hline & & 111.26 & & \\
\hline \multirow{2}{*}{$\begin{array}{l}\text { PACT50- } \\
\text { MIX }\end{array}$} & \multirow{2}{*}{57.66} & 12.81 & \multirow{2}{*}{0.002} & \multirow{2}{*}{$0-X$} \\
\hline & & 102.52 & & \\
\hline \multirow{2}{*}{$\begin{array}{l}\text { PINH10 - } \\
\text { MIX }\end{array}$} & \multirow{2}{*}{7.70} & -37.14 & \multirow{2}{*}{0.999} & \multirow{2}{*}{ - } \\
\hline & & 52.56 & & \\
\hline \multirow{2}{*}{$\begin{array}{l}\text { PINH50 - } \\
\text { MIX }\end{array}$} & \multirow{2}{*}{-1.58} & -46.43 & \multirow{2}{*}{1} & \multirow{2}{*}{-} \\
\hline & & 43.26 & & \\
\hline
\end{tabular}

\begin{tabular}{|c|c|c|c|}
\hline \multicolumn{4}{|c|}{$100 \mathrm{EXT}$} \\
\hline DIFF & $\begin{array}{l}\text { LWR } \\
\text { UPR }\end{array}$ & $P$ & RES \\
\hline \multirow{2}{*}{3.45} & -22.73 & \multirow{2}{*}{0.99} & \multirow{2}{*}{ - } \\
\hline & 29.65 & & \\
\hline \multirow{2}{*}{-23.83} & -50.02 & \multirow{2}{*}{0.106} & \multirow{2}{*}{-} \\
\hline & 2.35 & & \\
\hline \multirow{2}{*}{6.70} & -19.48 & \multirow{2}{*}{0.996} & \multirow{2}{*}{ - } \\
\hline & 32.90 & & \\
\hline \multirow{2}{*}{6.54} & -19.65 & \multirow{2}{*}{0.997} & \multirow{2}{*}{-} \\
\hline & 32.73 & & \\
\hline \multirow{2}{*}{23.37} & -2.81 & \multirow{2}{*}{0.122} & \multirow{2}{*}{ - } \\
\hline & 49.56 & & \\
\hline \multirow{2}{*}{-7.29} & -33.48 & \multirow{2}{*}{0.994} & \multirow{2}{*}{ - } \\
\hline & 18.90 & & \\
\hline \multirow{2}{*}{-11.20} & -37.40 & \multirow{2}{*}{0.917} & \multirow{2}{*}{$\begin{array}{lll}- & \\
-1\end{array}$} \\
\hline & 14.98 & & \\
\hline
\end{tabular}

Table 9.7: Table showing Tukey comparison (using R) between MIX and the other methods in runs with 100 cycles. The columm Diff indicates the difference between the means of the methods. Since this is a minimization problem, a negative value of Diff indicates that the first method performed better; whereas a positive value indicates that the second method had the best performance. Column $\mathrm{P}$ is the Tukey adjusted $p$ value, i.e., the result of the significance test for diff (considering multiple testing). The last column Res, indicates which of the methods was best. 'X-o' signifies that the first algorithm performed better; on its hand, 'o- $\mathrm{X}$ ' signifies that the second method was best; finally '-' indicates that the difference wasn't statisticaly significant to formulate an opinion. 


\begin{tabular}{|c|c|c|c|c|}
\hline & \multicolumn{4}{|c|}{1000 OVR } \\
\hline & \multirow{2}{*}{ DIFF } & LWR & \multirow{2}{*}{$P$} & \multirow{2}{*}{ RES } \\
\hline & & UPR & & \\
\hline \multirow[t]{2}{*}{ MIX - } & \multirow{2}{*}{-534.20833} & -753.91 & \multirow{2}{*}{0} & \multirow{2}{*}{$X-o$} \\
\hline & & -314.49 & & \\
\hline \multirow{2}{*}{$\begin{array}{l}\text { MIX - } \\
\text { ACT20 }\end{array}$} & \multirow{2}{*}{-608.291667} & -828.00 & \multirow{2}{*}{0} & \multirow{2}{*}{$\mathrm{X}-\mathrm{o}$} \\
\hline & & -388.58 & & \\
\hline \multirow[t]{2}{*}{ MIX - } & \multirow{2}{*}{9.0416667} & -210.66 & \multirow{2}{*}{1} & \multirow{2}{*}{ - } \\
\hline & & 228.75 & & \\
\hline \multirow[t]{2}{*}{ PACT1 - } & \multirow{2}{*}{770.791667} & 551.08 & \multirow{2}{*}{0} & \multirow{2}{*}{$o-X$} \\
\hline & & 990.50 & & \\
\hline \multirow[t]{2}{*}{ PACT50 - } & \multirow{2}{*}{410.916667} & 191.20 & \multirow{2}{*}{0.001} & \multirow{2}{*}{$o-X$} \\
\hline & & 630.62 & & \\
\hline \multirow[t]{2}{*}{ PINH10 - } & \multirow{2}{*}{25} & -194.71 & \multirow{2}{*}{0.999} & \multirow{2}{*}{ - } \\
\hline & & 244.71 & & \\
\hline \multirow[t]{2}{*}{ PINH50 - } & \multirow{2}{*}{-75.25} & -294.96 & \multirow{2}{*}{0.977} & \multirow{2}{*}{ - } \\
\hline & & 144.46 & & \\
\hline
\end{tabular}

\begin{tabular}{|c|c|c|c|}
\hline \multicolumn{4}{|c|}{1000 EXT } \\
\hline \multirow{2}{*}{ DIFF } & LWR & \multirow{2}{*}{$P$} & \multirow{2}{*}{ RES } \\
\hline & UPR & & \\
\hline \multirow{2}{*}{-13.66} & -105.56 & \multirow{2}{*}{0.99} & \multirow{2}{*}{ - } \\
\hline & 78.22 & & \\
\hline \multirow{2}{*}{-26.16} & -118.06 & \multirow{2}{*}{0.993} & \multirow{2}{*}{-} \\
\hline & 65.72 & & \\
\hline \multirow{2}{*}{-355.66} & -447.56 & \multirow{2}{*}{0} & \multirow{2}{*}{$\mathrm{X}-\mathrm{o}$} \\
\hline & -263.77 & & \\
\hline \multirow{2}{*}{35.45} & -56.435 & \multirow{2}{*}{0.953} & \multirow{2}{*}{-} \\
\hline & 127.35 & & \\
\hline \multirow{2}{*}{47.5} & -44.39 & \multirow{2}{*}{0.792} & \multirow{2}{*}{ - } \\
\hline & 139.39 & & \\
\hline \multirow{2}{*}{387.45} & 295.56 & \multirow{2}{*}{0} & \multirow{2}{*}{$\mathrm{o}-\mathrm{X}$} \\
\hline & 479.35 & & \\
\hline \multirow{2}{*}{417.87} & 325.98 & \multirow{2}{*}{0} & \multirow{2}{*}{$o-X$} \\
\hline & 509.76 & & \\
\hline
\end{tabular}

Table 9.8: Continuation of Tukey comparison (using R) between MIX and the other methods. The table shows results for 1000 cycles.

\begin{tabular}{|c|c|c|c|c|}
\hline & \multicolumn{4}{|c|}{10000 OVR } \\
\hline & DIFF & $\begin{array}{l}\text { LWR } \\
\text { UPR }\end{array}$ & $\mathrm{P}$ & RES \\
\hline \multirow{2}{*}{$\begin{array}{l}\text { MIX - } \\
\text { ACT1 }\end{array}$} & \multirow{2}{*}{-4393.33} & -5136.51 & \multirow{2}{*}{0} & \multirow{2}{*}{ X-o } \\
\hline & & -3650.15 & & \\
\hline \multirow{2}{*}{$\begin{array}{l}\text { MIX - } \\
\text { ACT20 }\end{array}$} & \multirow{2}{*}{-6344.79} & -7087.97 & \multirow{2}{*}{0} & \multirow{2}{*}{ X-o } \\
\hline & & -5601.61 & & \\
\hline \multirow{2}{*}{$\begin{array}{l}\text { MIX - } \\
\text { INH }\end{array}$} & \multirow{2}{*}{-246.04} & -989.22 & \multirow{2}{*}{0.97} & \multirow{2}{*}{ - } \\
\hline & & 497.13 & & \\
\hline \multirow{2}{*}{$\begin{array}{l}\text { PACT1 - } \\
\text { MIX }\end{array}$} & \multirow{2}{*}{5087.83} & 4344.65 & \multirow{2}{*}{0} & \multirow{2}{*}{ o-X } \\
\hline & & 5831.01 & & \\
\hline \multirow{2}{*}{$\begin{array}{l}\text { PACT50 - } \\
\text { MIX }\end{array}$} & \multirow{2}{*}{2775.79} & 2032.61 & \multirow{2}{*}{0} & \multirow{2}{*}{$\mathrm{o}-\mathrm{X}$} \\
\hline & & 3518.97 & & \\
\hline \multirow{2}{*}{$\begin{array}{l}\text { PINH10 - } \\
\text { MIX }\end{array}$} & \multirow{2}{*}{-91.91} & -735.52 & \multirow{2}{*}{0.99} & \multirow{2}{*}{-} \\
\hline & & 551.69 & & \\
\hline \multirow{2}{*}{$\begin{array}{l}\text { PINH50 - } \\
\text { MIX }\end{array}$} & \multirow{2}{*}{509.08} & -388.22 & \multirow{2}{*}{0.69} & \multirow{2}{*}{ - } \\
\hline & & 1406.39 & & \\
\hline
\end{tabular}

\begin{tabular}{|c|c|c|c|}
\hline \multicolumn{4}{|c|}{$10000 \mathrm{EXT}$} \\
\hline DIFF & $\begin{array}{l}\text { LWR } \\
\text { UPR }\end{array}$ & P & RES \\
\hline \multirow{2}{*}{45} & -355.16 & \multirow{2}{*}{0.99} & \multirow{2}{*}{ - } \\
\hline & 445.16 & & \\
\hline \multirow{2}{*}{69.45} & -330.71 & \multirow{2}{*}{0.99} & \multirow{2}{*}{ - } \\
\hline & 469.62 & & \\
\hline \multirow{2}{*}{-4991.25} & -5391.41 & \multirow{2}{*}{0} & \multirow{2}{*}{ X-o } \\
\hline & -4591.08 & & \\
\hline \multirow{2}{*}{14.16} & -386.00 & \multirow{2}{*}{1} & \multirow{2}{*}{ - } \\
\hline & 414.33 & & \\
\hline \multirow{2}{*}{157.12} & -243.04 & \multirow{2}{*}{0.95} & \multirow{2}{*}{ - } \\
\hline & 557.28 & & \\
\hline \multirow{2}{*}{4785.79} & 4385.62 & \multirow{2}{*}{0} & \multirow{2}{*}{ o-X } \\
\hline & 5185.96 & & \\
\hline \multirow{2}{*}{5536.45} & 5136.28 & \multirow{2}{*}{0} & \multirow{2}{*}{ o-X } \\
\hline & 5936.62 & & \\
\hline
\end{tabular}

Table 9.9: Continuation of Tukey comparison (using R) between MIX and the other methods. The table shows results for 10000 cycles. 


\begin{tabular}{|c|c|c|c|c|}
\hline & \multicolumn{4}{|c|}{100000 OVR } \\
\hline & \multirow{2}{*}{ DIFF } & LWR & \multirow{2}{*}{$\mathrm{P}$} & \multirow{2}{*}{ RES } \\
\hline & & UPR & & \\
\hline \multirow{2}{*}{$\begin{array}{l}\text { MIX - } \\
\text { ACT1 }\end{array}$} & \multirow{2}{*}{-44468.62} & -47115.13 & \multirow{2}{*}{0} & \multirow{2}{*}{ X-o } \\
\hline & & -41822.11 & & \\
\hline \multirow{2}{*}{$\begin{array}{l}\text { MIX - } \\
\text { ACT20 }\end{array}$} & \multirow{2}{*}{-63850.62} & -66497.13 & \multirow{2}{*}{0} & \multirow{2}{*}{ X-o } \\
\hline & & -61204.11 & & \\
\hline \multirow{2}{*}{$\begin{array}{l}\text { MIX - } \\
\text { INH }\end{array}$} & \multirow{2}{*}{-1049.58} & -3696.09 & \multirow{2}{*}{0.95} & \multirow{2}{*}{-} \\
\hline & & 1596.92 & & \\
\hline \multirow{2}{*}{$\begin{array}{l}\text { PACT1 - } \\
\text { MIX }\end{array}$} & \multirow{2}{*}{47433.37} & 44786.86 & \multirow{2}{*}{0} & \multirow{2}{*}{ o-X } \\
\hline & & 50079.88 & & \\
\hline \multirow{2}{*}{$\begin{array}{l}\text { PACT50 - } \\
\text { MIX }\end{array}$} & \multirow{2}{*}{28299.66} & 25653.15 & \multirow{2}{*}{0} & \multirow{2}{*}{ o-X } \\
\hline & & 30946.17 & & \\
\hline \multirow{2}{*}{$\begin{array}{l}\text { PINH10 - } \\
\text { MIX }\end{array}$} & \multirow{2}{*}{1897.91} & -748.59 & \multirow{2}{*}{0.38} & \multirow{2}{*}{-} \\
\hline & & 4544.42 & & \\
\hline \multirow{2}{*}{$\begin{array}{l}\text { PINH50 - } \\
\text { MIX }\end{array}$} & \multirow{2}{*}{265.61} & 266684 & \multirow{2}{*}{0.021} & \multirow{2}{*}{$\mathrm{o}-\mathrm{X}$} \\
\hline & & 270543 & & \\
\hline
\end{tabular}

\begin{tabular}{|c|c|c|c|}
\hline \multicolumn{4}{|c|}{$100000 \mathrm{EXT}$} \\
\hline \multirow{2}{*}{ DIFF } & LWR & \multirow{2}{*}{$\mathrm{P}$} & \multirow{2}{*}{ RES } \\
\hline & UPR & & \\
\hline \multirow{2}{*}{-124.58} & -1426.07 & \multirow{2}{*}{0.9} & \multirow{2}{*}{ - } \\
\hline & 1176.90 & & \\
\hline \multirow{2}{*}{1167.95} & -133.53 & \multirow{2}{*}{0.12} & \multirow{2}{*}{ - } \\
\hline & 2469.45 & & \\
\hline \multirow{2}{*}{-53206.91} & -54508.40 & \multirow{2}{*}{0} & \multirow{2}{*}{$\mathrm{X}-\mathrm{o}$} \\
\hline & -51905.42 & & \\
\hline \multirow{2}{*}{419.91} & -881.57 & \multirow{2}{*}{0.99} & \multirow{2}{*}{ - } \\
\hline & 1721.40 & & \\
\hline \multirow{2}{*}{27.83} & -4902.58 & \multirow{2}{*}{0.02} & \multirow{2}{*}{-} \\
\hline & 4957.58 & & \\
\hline \multirow{2}{*}{54115.83} & 52814.34 & \multirow{2}{*}{0} & \multirow{2}{*}{$o-X$} \\
\hline & 55417.32 & & \\
\hline \multirow{2}{*}{58555.33} & 57253.84 & \multirow{2}{*}{0} & \multirow{2}{*}{$0-X$} \\
\hline & 59856.82 & & \\
\hline
\end{tabular}

Table 9.10: Continuation of Tukey comparison (using R) between MIX and the other methods. The table shows results for 100000 cycles. 


\section{APPENDIX C: Survey on the Al model animating Where is Lourenço Marques?}


A survey was conducted with the live audience of the exhibition at the Tin Shed Gallery, at Goldsmiths, during the period from the 30th of November until the 14th of December. A population of 24 respondents were asked to rate on a scale from 1 to 10 the following questions:

- Q1 - The characters moving on the surface of the virtual world have autonomous behaviour.

- Q2 - The characters moving on the surface of the virtual world have agency and make individual decisions.

- Q3 - The interaction of the characters in the virtual world obeys to an internal and coherent logic.

- Q4 - The system of AI animating the characters is based on an ecosystem.

- Q5 - The behaviour of the characters is realistic (in a cartoon way).

- Q6 - The interaction of the characters in the virtual world illustrates social interactions in a city.

- Q7 - The interaction of the characters in the virtual world illustrates social interactions of humans in a city.

- Q8 - The behaviour of the characters is interesting.

- Q9 - Characters have a life cycle involving birth, reproduction and death.

Results from the interviews with the audience are shown below. 

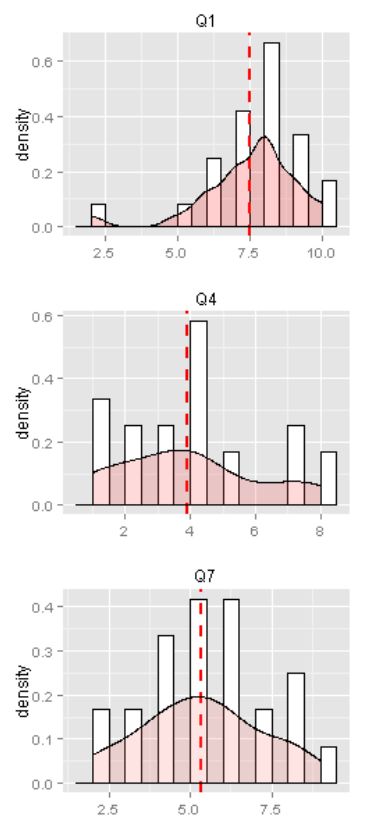

Q2

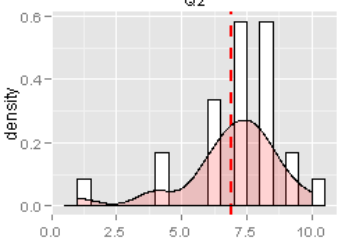

Q5
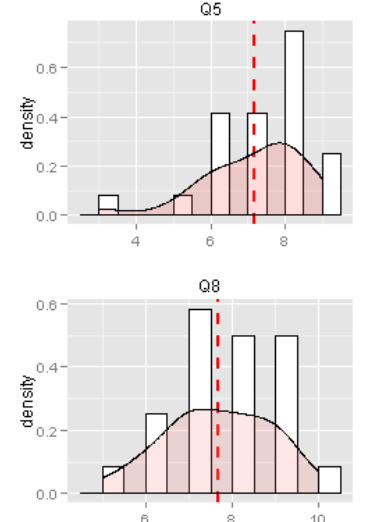

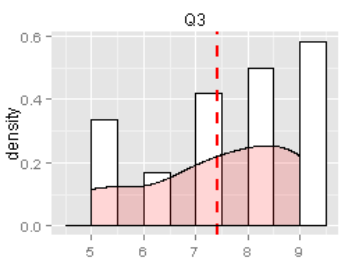

Q6

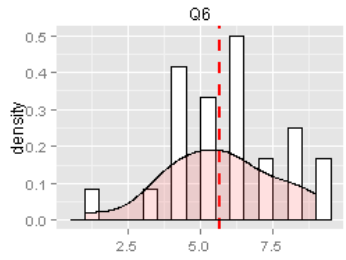

Q9

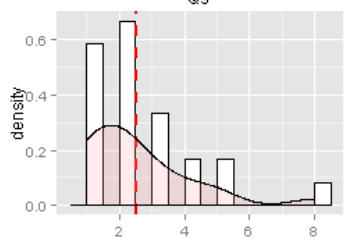

Figure 9.4: Histogram depicting results from interviews with the audience. From top to bottom and from left to right, the figures indicate the respective associated question. The dashed line indicates the mean; the overlaied kernel shows the density curve. The abscisses indicate the rating provided by the respondents in a scale from 1 to 10 . The axis of the ordinates indicate the density of the answers in the sample.

\begin{tabular}{|c|c|c|c|c|}
\hline Question & Mean & Standard error & \multicolumn{2}{|c|}{ Real mean } \\
\hline & & & $\min$ & $\max$ \\
\hline \hline Q1 & 7.5 & 0.3509821 & 7.149018 & 7.850982 \\
\hline Q2 & 6.916667 & 0.3850309 & 6.531636 & 7.301698 \\
\hline Q3 & 7.416667 & 0.2943715 & 7.122295 & 7.711038 \\
\hline Q4 & 3.916667 & 0.4501073 & 3.466559 & 4.366774 \\
\hline Q5 & 7.166667 & 0.2865757 & 6.880091 & 7.453242 \\
\hline Q6 & 5.666667 & 0.3977594 & 5.268907 & 6.064426 \\
\hline Q7 & 5.333333 & 0.3885437 & 4.94479 & 5.721877 \\
\hline Q8 & 7.666667 & 0.2530013 & 7.413665 & 7.919668 \\
\hline Q9 & 2.541667 & 0.3456729 & 2.195994 & 2.88734 \\
\hline
\end{tabular}

Table 9.11: Table summarizing the results of the survey. For each question the mean and standard errors are presented on the second and third columns respectively. The last two columns show the interval of the extrapolated real mean, calculated with $95 \%$ confidence. 
APPENDIX D: Public Exhibitions 


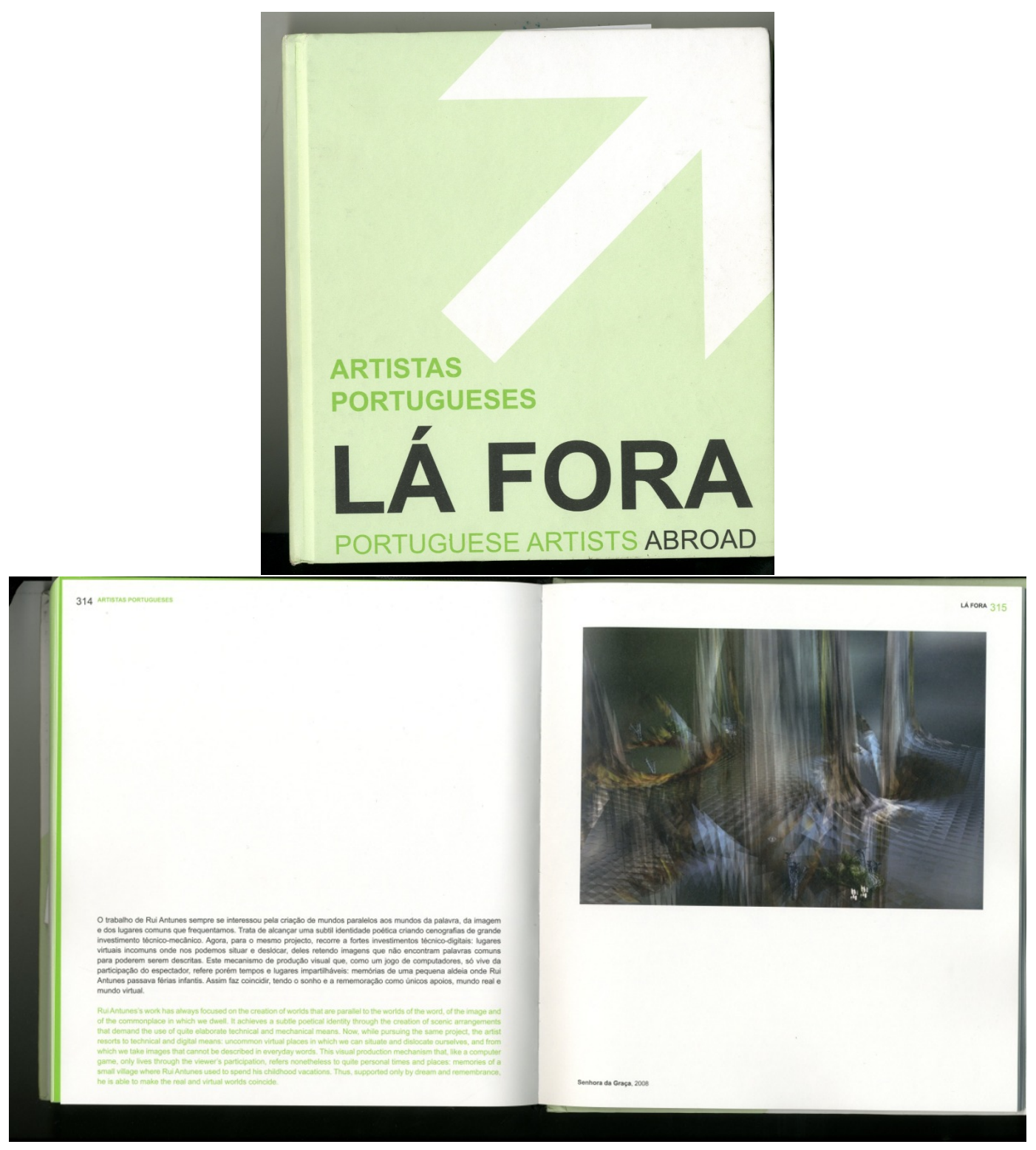

Figure 9.5: Images from the catalogue of the exhibition 'Lá fora', a perspective of Portuguese artists living and working abroad. The exhibition featured the work Senhora da Graça. 


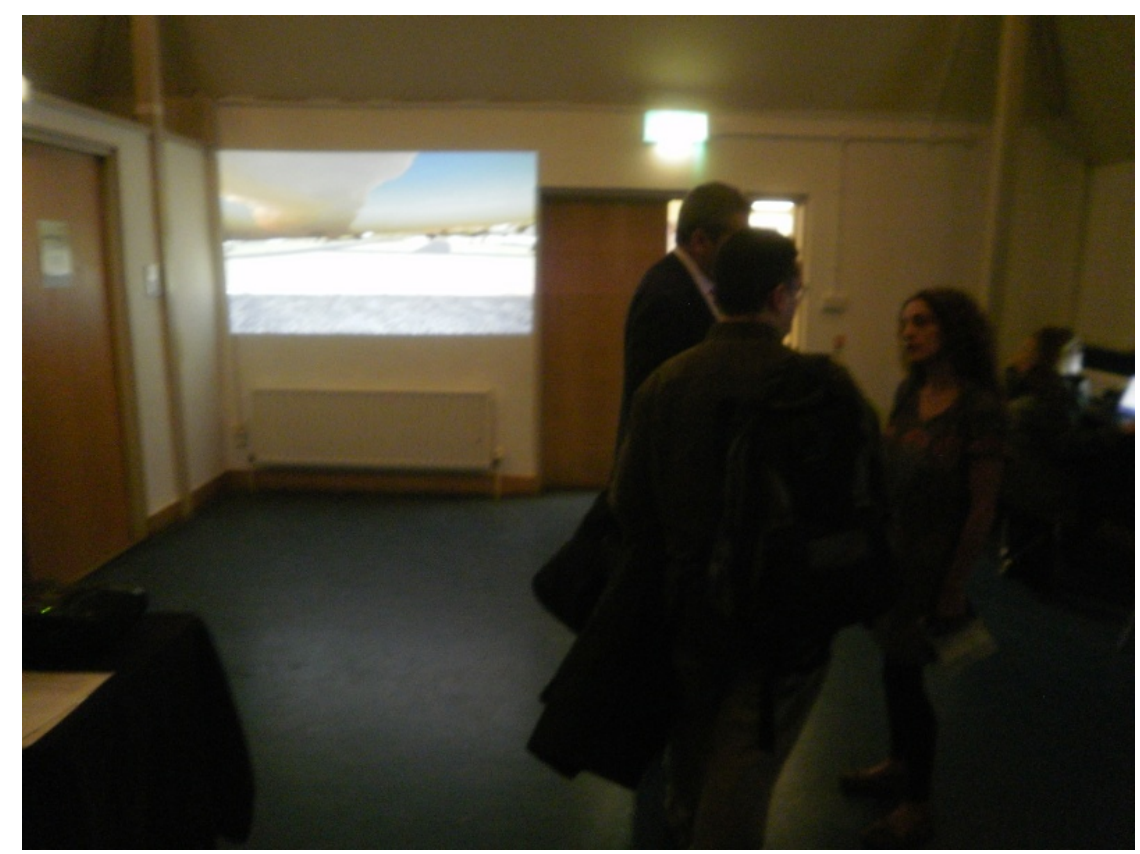

Figure 9.6: Image from the exhibition of WisLM at the closing ceremony of Watermans Festival of Digital Art 2012. I was part of the panel, 'Virtual space, Game space as Artistic Medium' - Chaired by Jonathan Munro - with Joseph Farbrook, Corrado Morgana, Parag K Mital, to talk about the work exhibited. 


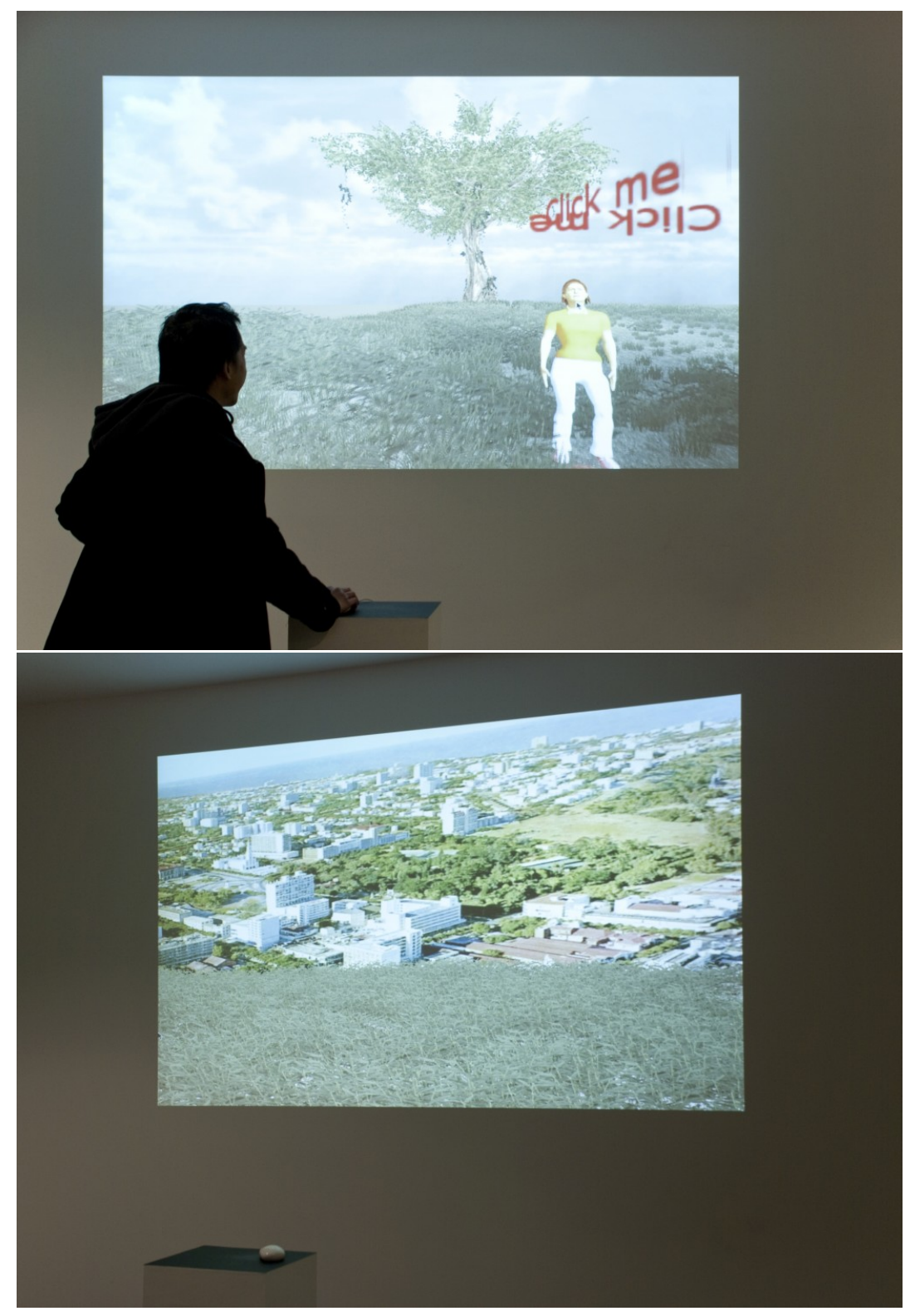

Figure 9.7: Images from the solo exhibition of WisLM at the Tin Shed, Goldsmiths from 29th November - 14th December 2012. The exhibition was accompained, in the opening session, by a panel with Amal Khalaf, Catarina Valdigem Pereira and myself on 'The role of collective memory in the construction of community identity and sense of place'. 
APPENDIX E: Multimedia Assets 
The following resources are available on the $\mathrm{CD}$ attached. For convenience I also made the same resources available online at the following links.

\section{Links to Videos:}

- Introduction to Where is Lourenço Marques?

http: //www . youtube. com/watch?v=Tp8lvdNWucw\&list=UUy43oDn0I3EHVaXQd2f s_Ww

- Summary of Senhora da Graça

http: //www . youtube. com/watch?v=pKIyq20ByNE\&list=UUy43oDn0I3EHVaXQd2fs_Ww

- Summary of Vishnu's Dance of Life and Death

http://www youtube.com/watch?v=YDZxkRz6pL4\&list=UUy43oDn0I3EHVaXQd2f s_Ww

The previous links can also be found at the research blog: http://www.ecoalife.com

\section{Links to Applications or source code:}

- Where is Lourenco Marques?

http://www. lourencomarques. net

- Senhora da Graca

http://www.lourencomarques.net/eco-alife/SraGraca.rar

- Vishnu's Dance of Life and Death

http://www.pikiproductions.com/rui/dancers/

- Model used in the Monte-Carlo study for the model EcoALife http://www. lourencomarques.net/eco-alife/Estatisticas-EcoALife.rar

\section{Visual Documentation of work in progress:}

http://www. lourencomarques. net/eco-alife/issu 